\title{
STRESS ECOLOGY OF THE PACIFIC RATTLESNAKES (CROTALUS OREGANUS AND CROTALUS HELLERI)
}

\author{
A Thesis \\ presented to \\ the Faculty of California Polytechnic State University,
}

San Luis Obispo

In Partial Fulfillment

of the Requirements for the Degree

Master of Science in Biological Sciences

by

Natalie May Claunch

June 2016 
(C) 2016

Natalie May Claunch

ALL RIGHTS RESERVED 


\section{COMMITTEE MEMBERSHIP}

TITLE: $\quad$ Stress Ecology of the Pacific Rattlesnakes (Crotalus oreganus and Crotalus helleri)

AUTHOR: $\quad$ Natalie May Claunch

DATE SUBMITTED: June 2016

COMMITTE CHAIR: $\quad$ Emily Taylor, Ph.D.

Associate Professor of Biology

COMMITTEE MEMBER: $\quad$ Clinton Francis, Ph.D.

Assistant Professor of Biology

COMMITEE MEMBER: $\quad$ Christy Strand, Ph.D.

Associate Professor of Biology 


\begin{abstract}
Stress Ecology of the Pacific Rattlesnakes (Crotalus oreganus and Crotalus helleri) Natalie May Claunch
\end{abstract}

Stress is a physiological state induced by disturbance or adverse environmental conditions and is modulated by the glucocorticoid hormone corticosterone (CORT) in reptiles. Stressors can have various impacts on vertebrate trait expression and may affect survival or reproduction. Little is known about the effects of chronically elevated CORT in free-ranging reptiles, or the effect of disturbance stress on venom composition in captive snakes.

In chapter 1, we investigated the effects of researcher induced disturbance on CORT levels and venom composition in a group of captive Northern Pacific rattlesnakes (Crotalus oreganus). Venom protein concentration and plasma CORT levels were compared before and after two weeks of unpredictable bouts of cage vibration, and to a non-vibrated control group. CORT levels were also assessed one week into vibration treatment. We found no effect of vibration treatment on CORT levels or on venom composition, and within-snake relative protein abundance was highly repeatable, although some variation was observed. We found a strong correlation between changes in relative abundance of several proteins and CORT. These results led us to believe that while differential forms of researcher-induced disturbance may not affect venom composition, significant changes in baseline CORT, or chronic stress, may affect the venom phenotype.

In the next study, we investigated the effects of chronically elevated CORT in a wild population of radio-telemetered Southern Pacific rattlesnakes $(C$. helleri). Snakes were implanted intra-coelomically with either crystalline CORT or sham implants. Prior to implant and for two week periods thereafter, we sampled blood, venom, defensive behavior, and body temperature $\left(\mathrm{T}_{\mathrm{b}}\right)$. Thermal data logger implants recorded snake $\mathrm{T}_{\mathrm{b}}$ each hour. Snakes were tracked daily for one month, and detectability, defensive behavior, movement, home range size and thermal parameters were calculated for each group during the periods between samples. Stress reactivity was assessed as change in CORT from baseline after one hour of acute confinement stress. CORT implants led to elevated baseline CORT for at least two weeks in treatment snakes, showing that our treatment was successful. Chapter 2 describes the effects of CORT treatment on venom parameters. Increased baseline CORT was associated with increased activity of venom protein phospholipase $\mathrm{A}_{2}$, indicating that CORT may have direct effects on regulating venom protein activity. Overall, venom activity was repeatable within individual snakes. Chapter 3 describes the effect of CORT on behavioral, ecological, and physiological variables. Implant treatment led to decreased average $T_{b}$ in weeks two and three. We detected a trend for lower baseline CORT to predict a greater magnitude of acute stress response. Snakes with higher testosterone levels exhibited higher defensive behavior scores. Overall, there were no other effects of implant treatment.

Our results suggest that rattlesnake thermoregulation is impacted by chronic stress, which could affect other aspects of their metabolism and ecology. Results of both studies suggest baseline CORT may direct both the activity and relative abundance of 
venom proteins in different manners, a hypothesis which deserves further investigation using proteomic tools. When responding to an acute stressor, rattlesnakes may secrete CORT until a threshold response is reached, regardless of baseline levels. Overall, rattlesnakes appear resilient to the effects of researcher-induced disturbance in the laboratory and to two weeks of chronically elevated CORT in the field, as no change was detected in many of the parameters investigated. 


\section{ACKNOWLEDGMENTS}

The work presented in this thesis would not have been possible without the support and guidance of many people.

First and foremost, I would like to thank my adviser and committee chair Emily Taylor for guiding me through my first independent research endeavor, and supporting my pursuits in a giant project while keeping it within the bounds of reality. She was always willing to attend to my many panics, and has been an excellent mentor in both the sciences and the gustatory delights of Central California.

Tony Frazier was essential to this project, and collected the venom samples. I could not have asked for a better mentor to train me in field techniques and in maintaining mental stability after long grueling hours of tracking snakes to empty holes.

Matt Holding kindly ran venom assays at Ohio State, and spent many a Skype session discussing data analysis and organization of the manuscripts. Thanks especially to Griffin Capehart for entertaining me in both the lab and the field, and for being willing to track snakes at a moment's notice. Mike DeLea helped me navigate many demands of graduate school. Andrew Schaffner was immensely helpful in teaching me the inner workings of statistical modeling with an appropriate dose of humor through our many meetings discussion non-significant results. Many thanks to Ignacio Moore who has performed hormone assays for our lab through the years, and to Camilo Escalón and Ben Vernasco for their assistance.

I would like to thank my fantastic committee members Dr. Christy Strand and Dr. Clint Francis for all of their feedback, assistance, and speed in returning comments on my thesis. My fellow graduate students deserve many thanks for many insightful discussions, especially Ben Davidson for always lending an ear and Jackie Campbell for enlightening me on the finer points of protein-regulation.

An army of undergraduates helped collect data for this thesis: Avery Finden, Caitlin Parker, Tori Mellard, Roxanne Miller, Daniel Lomayesva, Brooke Wainwright, Olivia Duner, Daniel Johnson, Mason Dubois, Nicole Durtschi, Kelly Paulsen, Katie King, Garrett Jordan, Brandi Bergreen, Daniel Namyst, Kat Burns, Sean Belingheri, Shannon Kitchell, Kristen Koyama, Anna Parker, Sean Sullivan, and John Stepanek,. The Allan Hancock Bridges to the Baccalaureate program funded undergraduates Josiah Close and Courtney Connolly whose assistance was instrumental in this project.

I am grateful for Kate McCurdy and University of California Santa Barbara's assistance in facilitating this project at the Sedgwick Reserve, and John Sayers with California State Parks from Montaña de Oro. I would like to acknowledge California Polytechnic State University, Cal Poly College Based Fees and travel funds, Virginia Tech, and Ohio State University, and the National Science Foundation for providing funding for different aspects of this project.

Lastly, I would like to thank my husband Patrick Miller, who served both as sugar daddy in funding the bulk of my tuition and as a therapist by mediating my CORT levels during the past two years of this Master's program. 


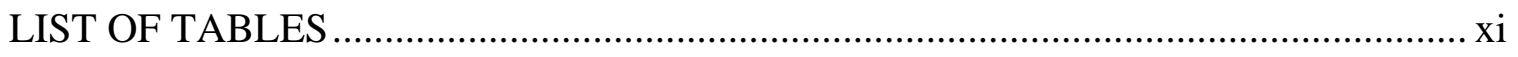

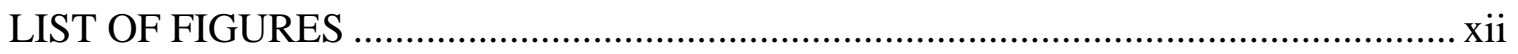

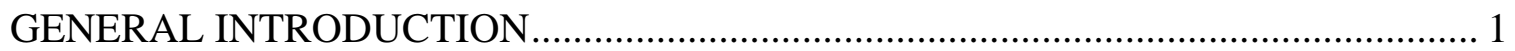

CHAPTER

I. EFFECTS OF RESEARCHER-INDUCED DISTURBANCE ON VENOM COMPOSITION OF CROTALUS OREGANUS ....................... 4

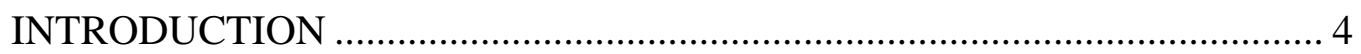

MATERIALS AND METHODS...................................................................... 7

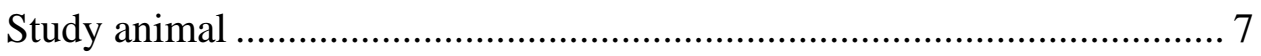

Researcher-induced disturbance ……………………….......................... 7

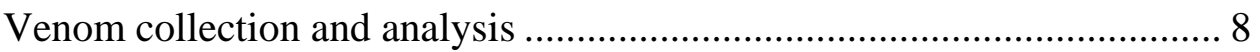

Blood sampling and hormone analysis .................................................. 10

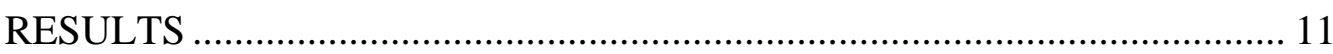

Vibration treatment and venom .............................................................. 11

Vibration treatment and CORT.......................................................... 12

CORT is associated with venom compositional change ............................ 13

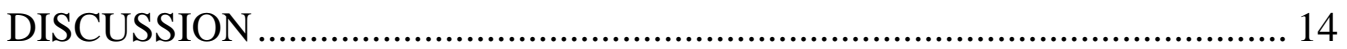

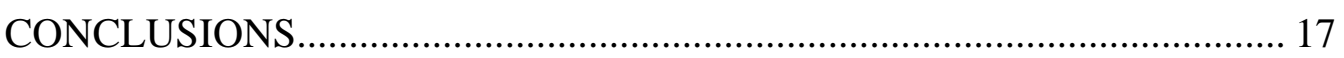

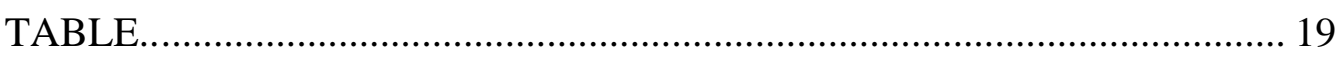

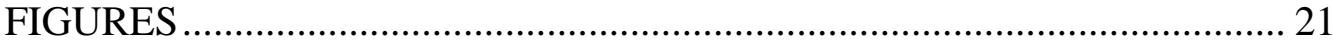

II. EFFECTS OF CORTICOSTERONE IMPLANTS ON CROTALUS HELLERI VENOM TOTAL PROTEIN CONCENTRATION AND

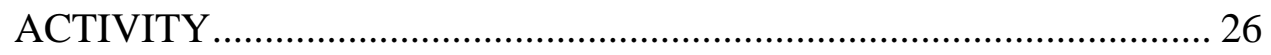

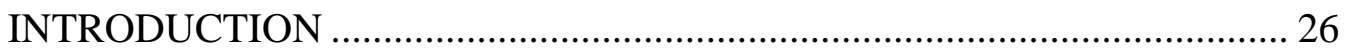

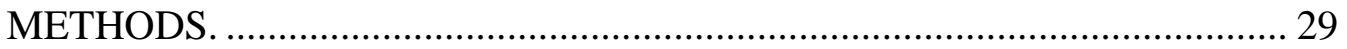

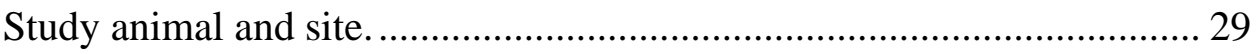

Experimental administration of exogenous corticosterone......................... 30

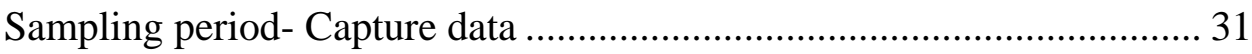

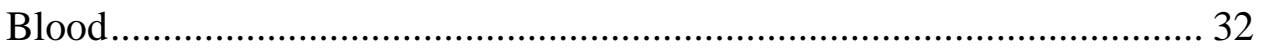

Radioimmunassay of blood plasma …………………………..... 32 
Venom 33

Venom protein concentration.......................................................... 34

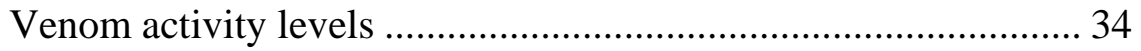

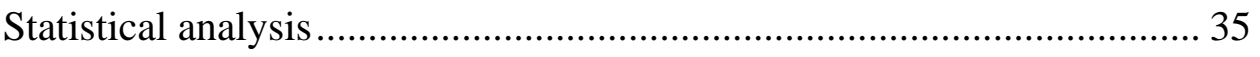

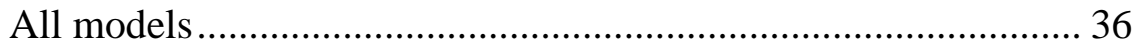

Baseline circulating CORT ………........................................... 37

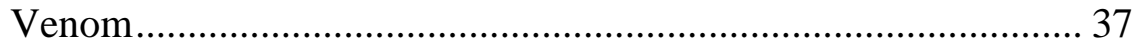

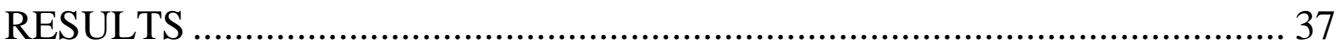

Implants elevated baseline CORT in treatment snakes............................. 37

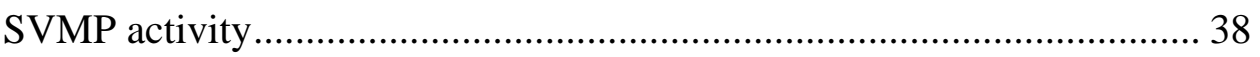

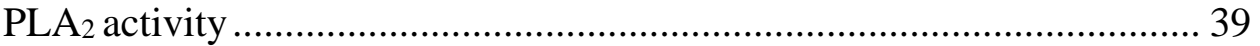

Total protein concentration of venom...................................................... 40

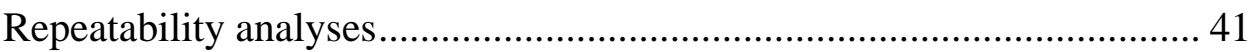

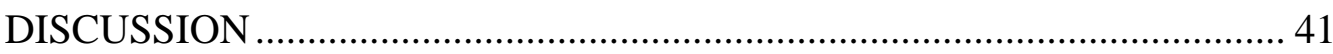

CORT implant treatment elevated blood CORT levels in

treatment snakes ....................................................................................... 41

Elevated CORT during synthesis did not affect venom

activity or total protein concentration ........................................................ 43

Baseline CORT is associated with increased PLA 2 activity ..................... 44

Effects of time and repeated sampling on protein activity

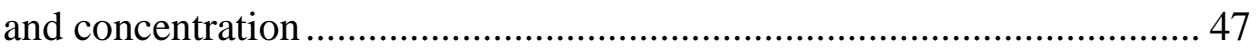

Association of snake size and SVMP activity adds

complexity to current literature

SVMP phenotype is related to difference in total SVMP

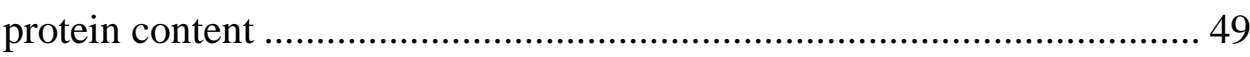

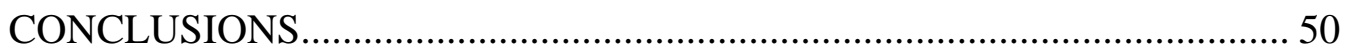

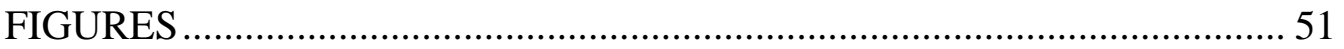

III. EFFECTS OF CORTICOSTERONE IMPLANTS ON PHYSIOLOGICAL, BEHAVIORAL, SPATIAL, AND THERMOREGULATORY ECOLOGY OF CROTALUS

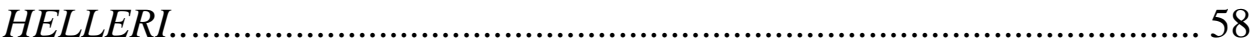

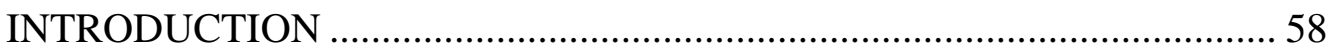


METHODS 61

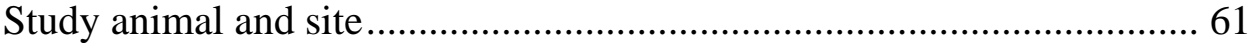

Experimental administration of exogenous corticosterone......................... 62

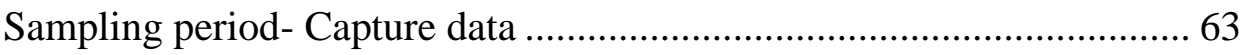

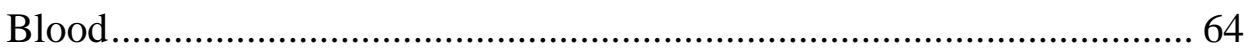

Radioimmunoassay of blood plasma ............................................ 65

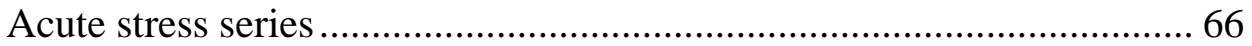

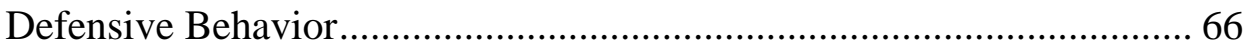

Defensive behavior during tracking period..................................... 67

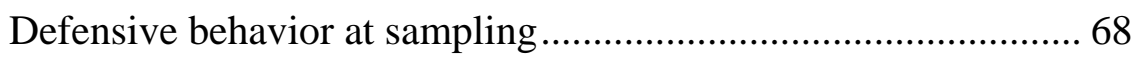

Movement, home range, detection probability ........................................... 68

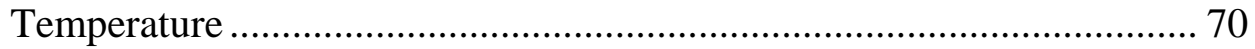

Model selection and organization of data ............................................... 71

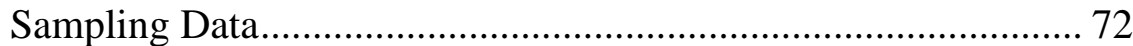

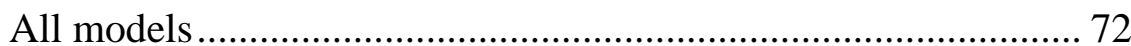

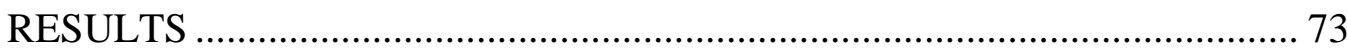

Effect of CORT implants on blood hormone levels .................................. 73

CORT levels were elevated by treatment at sample 2 .................... 73

Implants did not affect T levels ...................................................... 73

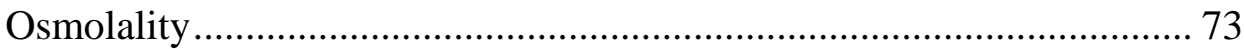

Trend for baseline CORT affecting magnitude of stress reactivity ......... 74

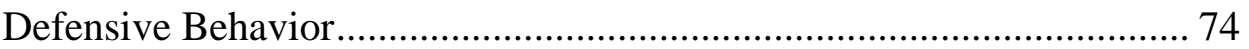

During tracking period ................................................................. 74

Defensive behavior assay during sampling....................................... 75

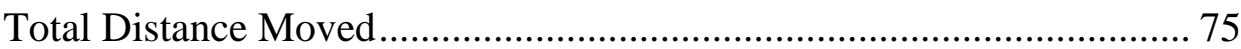

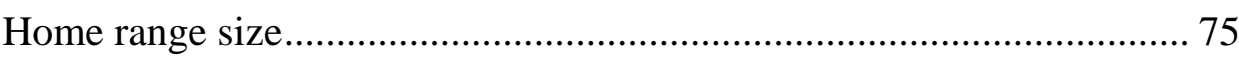

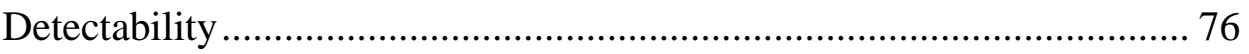

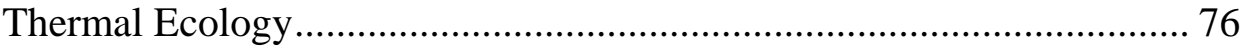

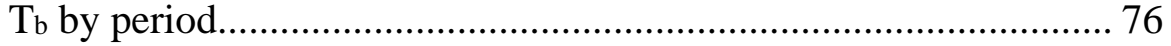

Hourly fluctuation by period......................................................... 76

Hourly temperature fluctuation by week .......................................... 77

Evaluating long term effects of CORT implants ....................................... 78 


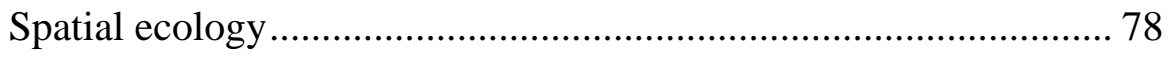

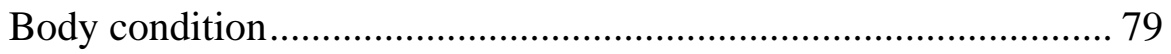

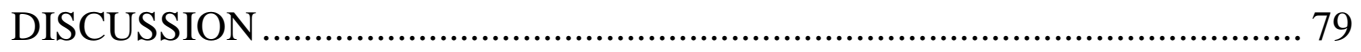

CORT implants elevated CORT in treatment snakes at

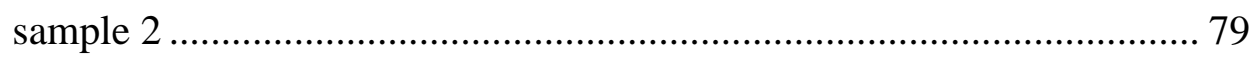

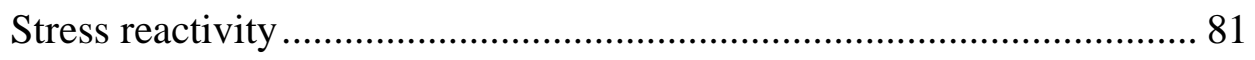

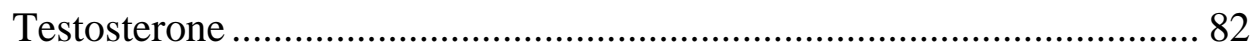

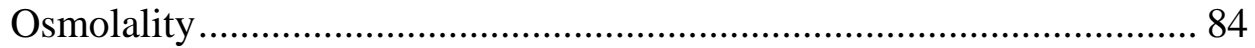

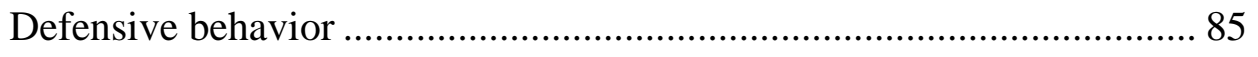

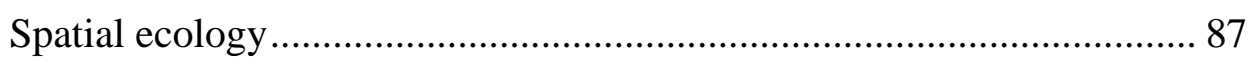

Body temperature $\left(\mathrm{T}_{\mathrm{b}}\right)$ and thermoregulation .......................................... 88

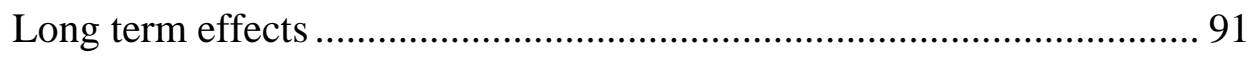

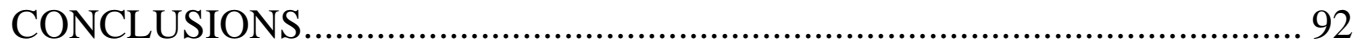

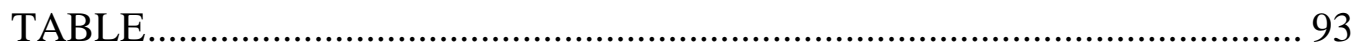

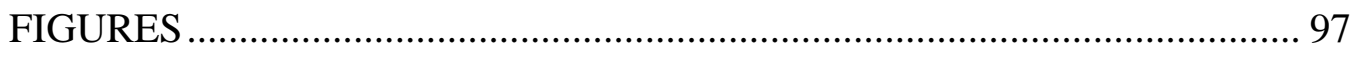

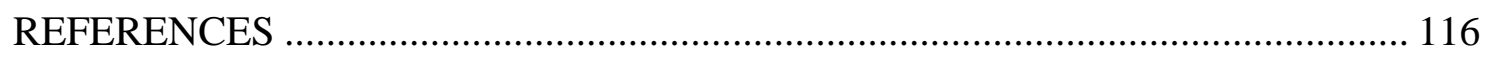

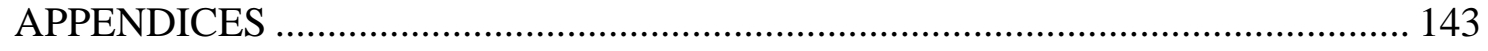




\section{LIST OF TABLES}

Table

Page

Table 1. Components 1 and 2 from principal component analysis of venom variation in Crotalus oreganus. The five highest loadings are bolded for component 1 ............... 19

Table 2. Results from various analyses. Significant factors and covariates are bolded. .. 93 


\section{LIST OF FIGURES}

Figure

Figure 1. Example vibrator device setup viewed from the back of the cages. Sonic Boom vibrator unit (A) is attached to the wooden ribs, which are fitted with plastic hooks that rest upon the back of the cage (B). The contraption is suspended via nylon string attached to the metal shelving unit $(\mathrm{C})$, and its vibrations are activated and controlled by the Alarm clock timer (D).

Figure 2. Representative HPLC chromatograms, showing venom profiles of control Crotalus oreganus subjected to captivity only (right) and a treatment snake subjected to vibration treatments (left) between the two venom extraction time points $($ pre-treatment $=$ black, post $=$ dotted red). Relative abundance of venom proteins are represented by individual peaks. The profiles overlap in a near-perfect fashion, indicating very little change in the relative abundance of the venom proteins within individuals' venoms over time, and no effect of vibration treatment.

Figure 3. Individual mean centered log-ratio transformed abundance for each RP-HPLC peak across vibration-treated and control Crotalus oreganus. The position on the $\mathrm{x}$-axis of each graph indicates the peak mean abundance during the first extraction of venom, and the position on the y-axis indicates that for the second extraction (post-treatment). High-expression proteins are represented by black circles, low-expression proteins by grey. Bars indicate one S.E.M., the solid line shows perfect agreement in peak relative abundance between initial and final sampling, and the dashed lines indicates the geometric mean of the dataset. Proteins with clr peak means below the geometric mean in both groups were classified as low expression proteins following Margres et al. (2016).

Figure 4. Plasma corticosterone (CORT) values of individual Crotalus oreganus at initial, 7 days post, and 15 days post vibration treatment. Black lines represent treatment snakes, gray lines control snakes. There was no effect of treatment or time on CORT levels $\left(\mathrm{F}_{2,6}=2.52, \mathrm{p}=0.13 ; \mathrm{F}_{2,6}=0.48, \mathrm{p}=0.63\right)$..

Figure 5. Association between movement through venom phenotype space and CORT for individual Crotalus oreganus. Biplot (left) of venom principle component 1 and 2, where the first and second venom samples from each snake are coded by the same symbol. Scatterplot (right) showing the association between the change in venom principle component 1 scores during captivity and the average baseline CORT concentration of individual control (circles) and vibration-treated (triangles) snakes $\left(\mathrm{R}^{2}=63.7 ; \mathrm{p}=0.02\right)$.

Figure 6. Baseline corticosterone (CORT) levels (ng/ml; +/- SEM) of control (light bars) and implant-treated (dark bars) Crotalus helleri across three sampling 
events. Baseline CORT was elevated in treatment snakes at the 15 day period after implant. Groups that do not share the same letter are significantly different.

Figure 7. Mean protein activities (A,B) and total protein concentration (C) of control (light bars) and CORT-implanted (dark bars) Crotalus helleri at each sampling event. There were no differences in SVMP activity based on treatment. SVMP activity was higher in sample 3 than the pre-implant sample. There were no differences in PLA 2 activity or total protein concentration based on treatment or repeated sampling. Asterisk indicates a significance difference. Error bars are \pm 1 SEM.

Figure 8. $\log _{10}$ transformed PLA2 activity of Crotalus helleri plotted against baseline CORT values across all three sampling periods (in sequence from light to dark). While there was no main effect of sampling period, baseline sampled CORT was a significant covariate in the RM ANCOVA, with activity increasing with baseline sampled CORT, regardless of treatment group or sample period. Although all three samples from each snake are shown, each within-snake sample was not considered independent. The RM ANCOVA accounted for repetition within individuals, so there was no pseudoreplication in our dataset.

Figure 9. Change in PLA2 activity from pre-implant sample to sample 2 in Crotalus helleri. Days between sampling events significantly impacted the changes in activity, driven by 3 snakes with samples greater than 20 days apart. With these excluded, the relationship was no longer significant.

Figure 10. Mean total protein concentration of Low SVMP activity phenotype (light hatched bars) and High SVMP activity phenotype (dark hatched bars) Crotalus helleri. High SVMP activity phenotype snakes had higher total protein concentration of venom at all timepoints. Different letters indicate significant differences between groups. Error bars are \pm 1 SEM.

Figure 11. Change in total protein concentration from pre-implant sampling to sample 2 after implant in Crotalus helleri. There was a positive (but not significant) trend for effect of days between sampling events on total protein concentration driven by three snakes with samples greater than 20 days apart. 56

Figure 12. Individual response of Crotalus helleri venom protein activity (A, B) and total protein concentration $(C)$ over time separated by treatment group. Each line depicts responses of an individual snake to repeated sampling across sampling periods. SVMP (A) and PLA2 (B) activity was highly repeatable within individual C. helleri, regardless of treatment. Total protein concentration was not reliably repeatable (C). Note obvious gap in activities between Log10 SVMP activity of 
2.3 and 3.3, leading to our designation of "low phenotype" and "high phenotype" respectively.

Figure 13. CORT plotted against days since implant administration (light= control, dark=CORT) for Crotalus helleri. Sample 2 fell between days 12-22. Sample 3 fell between days 27-35.

Figure 14. Individual CORT responses of Crotalus helleri to implantation of control (left) or CORT (right) implants across three samples. Each line represents one snake, while each point on the line represents a sampling event. Individual lines and points are shaded similar colors of gray.

Figure 15. Plasma T plotted against time of day for Crotalus helleri at each sample (coded sequentially from light to dark). There was a trend for $\mathrm{T}$ to decrease late in the day.

Figure 16. Plasma osmolality of treated (dark bars) and control (light bars) Crotalus helleri at each sampling period. Osmolality was not affected by treatment. Samples 2 and 3 for both groups had lower osmolality than the pre-implant samples. Groups marked with an asterisk are significantly different. Error bars are \pm 1 SEM.

Figure 17. Plasma osmolality plotted against time-to-bleed across all three sampling periods (coded sequentially from light to dark). Time-to-bleed was a significant covariate in the RM ANCOVA. Although all three samples from each snake are shown, each within-snake sample was not considered independent. The RM ANCOVA accounted for repetition within individuals, so there was no pseudoreplication in our dataset.

Figure 18. Stress reactivity of Crotalus oreganus after one hour of confinement in CORT-implanted (dark bars) and control snakes (light bars) at sampling period 3. Both groups showed a similar stress response, with no effect of treatment. Groups marked with an asterisk are significantly different. Error bars are \pm 1 SEM.

Figure 19. Magnitude of stress response in both control (light) and treatment (dark) Crotalus helleri after one hour of acute confinement stress. There was a trend for snakes with lower baseline CORT to exhibit a greater magnitude of CORT secretion in response to confinement stress than snakes with higher baseline CORT. 103

Figure 20. Relationship between change in $\mathrm{T}_{\mathrm{b}}$ and change in CORT in control (light) and treatment (dark) Crotalus helleri after one hour of acute confinement stress. There was a trend for snakes that increased in body temperature during confinement stress to exhibit a greater magnitude of CORT secretion. 104 
Figure 21. Average defensive behavior score during each tracking period for treatment (dark) and control (light) Crotalus helleri. Snakes with smaller ranges in $\mathrm{T}_{\mathrm{b}}$ were likely to have higher defensive behavior scores. Although all two datapoints from each snake are shown, each within-snake sample was not considered independent. The RM ANCOVA accounted for repetition within individuals, so there was no pseudoreplication in our dataset.

Figure 22. Average daily range in $\mathrm{T}_{\mathrm{b}}$ plotted against average $\mathrm{T}_{\mathrm{b}}$ for Crotalus helleri for each period. Range in $\mathrm{T}_{\mathrm{b}}$ strongly correlated to average $\mathrm{T}_{\mathrm{b}}$, with decreased range in $\mathrm{T}_{\mathrm{b}}$ associated with higher average $\mathrm{T}_{\mathrm{b}}$. Although two datapoints from each snake are shown, each within-snake sample was not considered independent. The RM ANCOVA accounted for repetition within individuals, so there was no pseudoreplication in our dataset. 106

Figure 23. Average defensive behavior score during sampling of Crotalus helleri across three sampling periods (coded sequentially from light to dark).

Testosterone was a significant covariate in the RM ANCOVA, with increasing Testosterone correlated with an increased defensive behavior score. Although all three samples from each snake are shown, each within-snake sample was not considered independent. The RM ANCOVA accounted for repetition within individuals, so there was no pseudoreplication in our dataset. 107

Figure 24. Spatial ecology parameters across each post-implant period in control (light bars) and treatment (dark bars) Crotalus helleri. Post-implant period 1: 0-15 days; Post-implant period 2: 15-20 days. Total distance moved (A), total area covered as calculated from $\log 100 \mathrm{MCP}(\mathrm{B})$, and Detectability (C) were all unaffected by treatment. All snakes moved more (A), covered more area (B) and were more detectable in period 1 than period 2 (C). Groups marked with an asterisk are significantly different. Error bars are \pm 1 SEM 108

Figure 25. Total distance moved plotted against body condition of Crotalus helleri for each sampling period. There was a trend for an interaction between sampling period and body condition, where snakes were unaffected by body condition in period 2. Body condition was a significant covariate, with snakes in lower body condition moving more. Although two datapoints from each snake are shown, each within-snake sample was not considered independent. The RM ANCOVA accounted for repetition within individuals, so there was no pseudoreplication in our dataset.

Figure 26. Average daily thermal parameters for Maximum $\mathrm{T}_{b}(\mathrm{~A})$. Minimum $\mathrm{T}_{\mathrm{b}}$ (B), Average $\mathrm{T}_{\mathrm{b}}(\mathrm{C})$, Range in $\mathrm{T}_{\mathrm{b}}$ (D) for treatment (dark bars) and control (light bars) Crotalus helleri at each sampling period. Maximum $\mathrm{T}_{\mathrm{b}}$ was unaffected by treatment or period. Minimum $\mathrm{T}_{\mathrm{b}}$ and Average $\mathrm{T}_{\mathrm{b}}$ were unaffected 
by treatment, but all snakes had lower minimum and average $T_{b}$ at pre-implant sampling compared to sample 2 . Range in $\mathrm{T}_{\mathrm{b}}$ was unaffected by treatment, but all snakes had greater range in $\mathrm{T}_{\mathrm{b}}$ at pre-implant sampling compared to sample 1 and 2. Groups marked with an asterisk are significantly different. Error bars are \pm 1 SEM

Figure 27. Average daily fluctuation in body temperature for control (open circles) and treatment (closed circles) Crotalus helleri at each sampling period. Maximum body temperatures reached were similar for each sampling period, while minimum temperatures and inflection points were higher in period 2 than pre-implant, and a few cool-down temperatures were different in period 1 than pre-implant. Period 2 and 1 were not significantly different from one another. Asterisks indicate significant differences compared to the same hours during the Pre-implant period. Error bars are \pm 1 SEM.

Figure 28. Average $\mathrm{T}_{\mathrm{b}}$ for treatment (dark bars) and control (light bars) Crotalus helleri for the week before implant and four weeks post-implant. Treatment snakes pre-implant had higher $\mathrm{T}_{\mathrm{b}}$, this effect was reversed in weeks 2 and 3 postimplant. Asterisks indicate significant differences between treatment groups. Error bars are \pm 1 SEM.

Figure 29. Home range size of control (light) and treatment (dark) Crotalus helleri as measured with $95 \%$ MCPs. There was no effect of treatment on home range size, but initial body condition at implantation was a significant covariate in the RM ANCOVA, with snakes in poorer body condition having larger home ranges.

Figure 30. Percent change in body condition from implant date for Crotalus helleri. All snakes decreased in body condition over the study, but there was no difference in body condition based on treatment in the one-way ANOVA. Error bars are \pm 1 SEM

Figure 31. Percent change in body condition from implant date to end of study for control (light) and treatment (dark) Crotalus helleri. SVL was marginally significant, with a trends for smaller snakes to have greater negative change in body condition.

Figure A1. $\log _{10}$ transformed SVMP activity of Crotalus helleri plotted against baseline CORT values across all three sampling periods (coded sequentially from light to dark). There was no relationship between baseline sampled CORT and SVMP activity in the RM ANCOVA. Although all three samples from each snake are shown, each within-snake sample was not considered independent. The 
RM ANCOVA accounted for repetition within individuals, so there was no pseudoreplication in our dataset

Figure A2. Log10 transformed SVMP activity of High SVMP phenotype (crosses) and Low SVMP phenotype (open circles) Crotalus helleri across the three sampling periods (in sequence from light to dark). SVL was not a significant covariate in the full model. Note opposing pattern between phenotypes and SVL. Note also that clusters of points along the $\mathrm{x}$-axis represent the samples from the same individual snake as SVL did not change appreciably during the study. Although all three samples from each snake are shown, each within-snake sample was not considered independent. The RM ANCOVA accounted for repetition within individuals, so there was no pseudoreplication in our dataset.

Figure A3. $\log _{10}$ transformed SVMP activity of High SVMP activity phenotype Crotalus helleri plotted against baseline CORT values across all three sampling periods (in sequence from light to dark). CORT was not a significant covariate. Although all three samples from each snake are shown, each within-snake sample was not considered independent. The RM ANCOVA accounted for repetition within individuals, so there was no pseudoreplication in our dataset.

Figure A4. $\log _{10}$ transformed SVMP activity of Low SVMP activity phenotype Crotalus helleri plotted against baseline CORT values across all three sampling periods (in sequence from light to dark). CORT was not a significant covariate. Although all three samples from each snake are shown, each within-snake sample was not considered independent. The RM ANCOVA accounted for repetition within individuals, so there was no pseudoreplication in our dataset. 146

Figure A5. Mean Log10 transformed SVMP activity of High SVMP Phenotype control (light bars) and CORT-implanted (dark bars) Crotalus helleri. There was no effect of treatment or repeated sampling in the High SVMP Phenotype group on SVMP activity. Error bars are \pm 1 SEM.

Figure A6. Mean Log10 transformed SVMP activity of Low SVMP Phenotype control (light bars) and CORT-implanted (dark bars) Crotalus helleri. There was no effect of treatment or repeated sampling in the Low SVMP Phenotype group on SVMP activity. Error bars are \pm 1 SEM.

Figure A7. $\log _{10}$ transformed SVMP activity of High SVMP activity phenotype Crotalus helleri plotted against SVL values across all three sampling periods. SVL was a significant covariate in the RM ANCOVA, with larger High Phenotype snakes tending to have higher SVMP activity. Although all three samples from each snake are shown, each within-snake sample was not 
considered independent. The RM ANCOVA accounted for repetition within individuals, so there was no pseudoreplication in our dataset.

Figure A8. $\log _{10}$ transformed SVMP activity of Low SVMP activity phenotype Crotalus helleri plotted against SVL across all three sampling periods (in sequence from light to dark). SVL was not a significant covariate. Although all three samples from each snake are shown, each within-snake sample was not considered independent. The RM ANCOVA accounted for repetition within individuals, so there was no pseudoreplication in our dataset.

Figure A9. Mean change in SVMP activity from pre-implant sampling to sample 2 in control and CORT-implanted Crotalus helleri. Both control and treatment snakes showed overall average increases in SVMP activity during the initial implant period. There was no effect of treatment on change in SVMP activity in an ANCOVA. Error bars are \pm 1 SEM.

Figure A10. Change in SVMP activity from pre-implant sampling to sample 2 in Crotalus helleri. Days between sampling events was not a significant covariate.

Figure A11. Change in SVMP activity from pre-implant sampling to sample 2 in Crotalus helleri against change in baseline CORT. Change in baseline CORT between the samples was not a significant covariate.

Figure A12. Change in SVMP activity from pre-implant sampling to 15 day after implant period in Crotalus helleri. SVL was not a significant covariate.

Figure A13. Change in SVMP activity from pre-implant sampling to sample2 in Low and High SVMP activity phenotype Crotalus helleri. Both phenotypes showed average increases in SVMP activity, but there was no effect of SVMP phenotype on change in SVMP activity during the initial implant period. Error bars are \pm 1 SEM.

Figure A14. Log10 transformed PLA2 activity of Crotalus helleri plotted against SVL across all three sampling periods (in sequence from light to dark). SVL was not a significant covariate in the RM ANCOVA. Note clusters of dots along the $\mathrm{X}$-axis represent the samples from the same individual snake as SVL did not change appreciably during the study. Although all three samples from each snake are shown, each within-snake sample was not considered independent. The RM ANCOVA accounted for repetition within individuals, so there was no pseudoreplication in our dataset.

Figure A15. Histogram showing mean change in PLA2 activity from pre-implant sampling to the second period (approximately 15 days after implants) in control and CORT-treated Crotalus helleri. Both control and treatment groups showed 
overall average increases in activity over the period. There was no effect of treatment on change in PLA2 activity in an ANCOVA. Error bars are \pm 1 SEM

Figure A16. Change in PLA2 activity from pre-implant sampling to 15 days after implant period in Crotalus helleri. Change in baseline CORT between the samples was not a significant covariate.

Figure A17. Change in PLA2 activity from pre-implant sampling to sample 2 in Crotalus helleri. SVL was not a significant covariate.

Figure A18. Change in PLA2 activity from pre-implant sampling to sample 2 in Crotalus helleri. Initial body condition was a significant covariate, with snakes in higher body condition tending to decrease in PLA2 activity from pre-implant to second sampling.

Figure A19. Change in PLA2 activity from pre-implant sampling to sample 2 in Crotalus helleri, excluding three snakes with greater than 20 days between sampling events. Days between sampling was no longer a significant covariate

Figure A20. Total protein concentration in Crotalus helleri plotted against SVL across all three time periods (in sequence from light to dark). There was no relationship between SVL and total protein concentration. Note clusters of dots along the $\mathrm{x}$-axis represent the samples from the same individual snake as SVL did not change appreciably during the study. Although all three samples from each snake are shown, each within-snake sample was not considered independent. The RM ANCOVA accounted for repetition within individuals, so there was no pseudoreplication in our dataset

Figure A21. Total protein concentration of Crotalus helleri plotted against baseline CORT values across all three sampling periods (colored sequentially from light to dark). CORT was not a significant covariate in the RM ANCOVA. Although all three samples from each snake are shown, each within-snake sample was not considered independent. The RM ANCOVA accounted for repetition within individuals, so there was no pseudoreplication in our dataset.

Figure A22. Histogram showing mean change in total protein concentration from pre-implant sampling to sample 2 (approximately 15 days after implants) in control and CORT-treated Crotalus helleri. While Control snakes showed overall average increases in total protein concentration and treatment snakes showed overall average decreases, there was no effect of treatment on change in total protein concentration in an ANCOVA. Error bars are \pm 1 SEM. 164 
Figure A23. Change in total protein concentration from pre-implant sampling to sample 2 after implant in Crotalus helleri. Change in baseline CORT between samples was not a significant covariate.

Figure A24. Change in total protein concentration from pre-implant sampling to sample 2 in Crotalus helleri. Initial body condition was not a significant covariate... 166

Figure A25. Change in total protein concentration from pre-implant sampling to sample 2 in Crotalus helleri. SVL was not a significant covariate.

Figure A26. Plasma osmolality of Crotalus helleri plotted against baseline CORT across all three sampling periods (coded sequentially from light to dark). CORT was not a significant covariate in the RM ANCOVA. Although all three samples from each snake are shown, each within-snake sample was not considered independent. The RM ANCOVA accounted for repetition within individuals, so there was no pseudoreplication in our dataset. 168

Figure A27. Mean plasma T of treatment (dark bars) and control (light bars) Crotalus helleri at each sampling period. T was not affected by treatment or sample number. Error bars are \pm 1 SEM.

Figure A28. Plasma testosterone plotted against time of day for Crotalus helleri at each sample (coded sequentially from light to dark). There was a trend for testosterone to decrease late in the day. Although all three samples from each snake are shown, each within-snake sample was not considered independent. The RM ANCOVA accounted for repetition within individuals, so there was no pseudoreplication in our dataset.

Figure A29. T plotted against CORT for Crotalus helleri across all three sampling periods (coded sequentially from light to dark). CORT was not a significant covariate in the RM ANCOVA. Although all three samples from each snake are shown, each within-snake sample was not considered independent. The RM ANCOVA accounted for repetition within individuals, so there was no pseudoreplication in our dataset

Figure A30. Magnitude of stress response in both control and treatment Crotalus helleri after one hour of acute confinement stress. There was no difference in stress response based on implant treatment. Error bars are \pm 1 SEM. 172

Figure A31. Average defensive behavior score during each tracking period for treatment (dark bars) and control (light bars) Crotalus helleri. Defensive behavior was not affected by treatment or sample number. Error bars are \pm 1 SEM. 
Figure A32. Average defensive behavior score during sampling for treatment (dark bars) and control (light bars) Crotalus helleri. Defensive behavior was not affected by treatment or sample number. Error bars are \pm 1 SEM.

Figure A33. Average defensive behavior score during sampling of Crotalus helleri across three sampling periods (coded sequentially from light to dark). CORT was not a significant covariate in the RM ANCOVA. Although all three samples from each snake are shown, each within-snake sample was not considered independent. The RM ANCOVA accounted for repetition within individuals, so there was no pseudoreplication in our dataset.

Figure A34. Average defensive behavior score during sampling of Crotalus helleri across three sampling periods (coded sequentially from light to dark). $\mathrm{T}_{\mathrm{b}}$ was not a significant covariate in the RM ANCOVA. Although all three samples from each snake are shown, each within-snake sample was not considered independent. The RM ANCOVA accounted for repetition within individuals, so there was no pseudoreplication in our dataset.

Figure A35. TDM of Crotalus helleri during the pre-implant tracking period. TDM was not significantly different based on treatment group in the one-way ANOVA. Error bars are \pm 1 SEM

Figure A36. Home range size as measured by $100 \% \mathrm{MCP}$ for control (light) and treatment (dark) Crotalus helleri over a three month period after implantation. There were no differences based on treatment. Error bars are \pm 1 SEM.

Figure A37. Home range size as measured by 95\% MCP for control (light) and treatment (dark) Crotalus helleri over a three month period after implantation. There were no differences based on treatment. Error bars are \pm 1 SEM. 


\section{GENERAL INTRODUCTION}

Stress affects many organisms, and is defined broadly as a state an organism experiences in coping with unpredictable or adverse conditions (Wingfield 2005). Stress can also be defined physiologically by the elevation of glucocorticoid hormone levels in response to such changes (Sapolsky, Romero \& Munck 2000). Within these broad definitions, stress can be further split into acute, short term responses, and chronic, sustained responses, with variation in effects based on the level of response (Dickens and Romero 2013). Acute responses are generally mounted during encounters with predators or aggressive conspecifics (Sapolsky, Romero \& Munck 2000), while chronic responses are often due to prolonged unfavorable environmental conditions including famine (Wingfield et al. 1998; Busch \& Hayward 2009). Chronic stress influences expression of a suite of traits in many species, including but not limited to reproduction (Wingfield \& Sapolsky 2003; Jones \& Bell 2004; Spée et al. 2011), immune function (Dhabhar 2000; Martin 2009; Seddon \& Klukowski 2012; McCormick \& Langkilde 2014), behavior (Baird, Lovern \& Shine 2014), thermoregulation (Cree et al. 2003; Preest \& Cree 2008), and protein metabolism (Romero, Strochlic \& Wingfield 2005; Lorenz et al. 2009) and does not necessarily produce consistent effects within a taxon (Cockrem 2013). Therefore, it is important to evaluate chronic stress in the context of individual species and populations for stress metrics to be useful in conservation biology (Breuner, Delehanty \& Boonstra 2013). Stress responses and glucocortoicoid levels are often used to assess the status of free-ranging populations of animals to predict future growth and reproduction, although the majority of studies focus on acute stress responses of

endothermic vertebrates (Busch \& Hayward 2009; Baker, Gobush \& Vynne 2013; 
Jessop, Woodford \& Symonds 2013). Fewer studies focus on the effects of chronic stress, which can be induced by sustained periods of unfavorable environmental conditions, such as drought (Wingfield et al. 1998).

In ectothermic vertebrates, such as reptiles, chronic stress may have different effects than in endotherms due to differences in metabolic rates. Ectotherms may endure chronic CORT elevation for longer periods of time than endotherms due to a generally slower metabolism (Landys et al. 2006). Despite this, evaluation of chronic elevation of CORT in terrestrial ectotherms has been limited (Miles et al. 2007; Cash \& Holberton 1999), and few studies have attempted to evaluate the effects of chronic stress in freeranging reptiles (DeNardo \& Licht 1993; DeNardo \& Sinervo 1994; Juneau et al. 2015).

We evaluated the effects of chronic stress on Pacific rattlesnake species (Crotalus oreganus and C. helleri), as these species are known to endure periods of low food availability and drought (McCue 2007). Rattlesnakes are also resilient to manipulation in the field (Taylor et al. 2005; Holding et al. 2014), such that observed responses are likely representative of treatment, rather than an effect of researcher presence. This thesis describes how the venoms and several behavioral, ecological, and physiological variables of rattlesnakes are affected, including both laboratory and field studies. We were interested in evaluating the effects of chronic stress in the context of unpredictable environmental disturbance, as well as in a physiological context through manipulation of CORT levels. In Chapter 1, we present the effects of researcher-induced disturbance on venom composition in captive male and female $C$. oreganus. Chapters 2 and 3 focus on the effects of CORT implantation in a radio-telemetered free-ranging population of male C. helleri. Chapter 2 evaluates the effects of chronic CORT on venom activity and total 
protein concentration. Chapter 3 evaluates the effects of chronic CORT on other aspects of rattlesnake ecology including physiology, behavior, spatial ecology, and thermal biology. Major tables and figures are included within the corresponding chapter, and supplementary tables and figures are included in a common Appendix for reference.

Rattlesnake response to disturbance and elevated CORT is important to understand from an ecological perspective to better understand how these organisms interact with a changing environment. To date, no studies have evaluated the effects of chronic stress in a free-ranging snake. Rattlesnake stress ecology is also important to understand from a medical perspective to evaluate potential changes in venom efficacy to inform treatment of snakebite and antivenom production (Calvete et al., 2009; Casewell et al. 2014; Núñez et al. 2009; Saviola et al. 2015), as well as to record spatial, thermoregulatory, and behavioral effects induced by chronic stress that may help in predicting snakebite incidence and morbidity for a given year (Akani et al. 2013; YañezArenas et al. 2016). 


\section{CHAPTER I: EFFECTS OF RESEARCHER-INDUCED DISTURBANCE ON VENOM COMPOSITION OF CROTALUS OREGANUS}

\section{INTRODUCTION}

Phenotypic plasticity contributes to intraspecific variation in many key traits organisms use to interact directly with their surroundings. The resulting trait variation can be adaptive for coping with temporally varying environmental challenges, such as the availability of key food sources, the presence and density of predators, the seasonal changes in energy budgets associated with mating or migration, and climatic fluctuations that directly or indirectly impact physiological performance (Pigliucci 2001).

Characterizing the changes in organismal phenotypes in response to changing demands or stressors can provide insights into the biological significance of phenotypic plasticity for a given trait, as well as establish a trait's repeatability during measurements taken over time.

The venom phenotype of snakes varies markedly within a species, with biological and biomedical consequences. Phenotypic plasticity of venom composition could contribute to functional variation in prey capture and digestion (Gibbs \& Mackessy 2009; Mackessy 1988), differential morbidity of snakebite in humans (Casewell et al. 2014; Massey et al. 2012; Calvete et al. 2011; Wasserberger et al. 2006; Bush et al. 2002), and the efficacy of antivenom used in treating snakebites from a single species (Calvete et al., 2009; Casewell et al. 2014; Núñez et al. 2009; Saviola et al. 2015). Most known instances of plasticity occur as ontogenetic shifts in venom protein composition, where venoms tend to shift from neurotoxic to myotoxic and proteolytic as snakes age (Alape-Girón et al. 2008; Mackessy et al. 1988; Saviola et al. 2015). These shifts are considered adaptive in that they mirror changes in diet that occur as snakes grow larger (Mackessy 1996). 
Current evidence for phenotypic plasticity in adult snake venoms is mixed. In captive rattlesnakes, seasonal changes in temperature or photoperiod did not alter venom composition measured through 1D gel banding patterns (Dwyer et al. 1986). To the contrary, two bands disappeared completely from pooled samples of sand vipers (Vipera ammodytes) during the summer (Gubensek et al., 1974), and monthly venom extraction of a single Eastern Brown Snake (Pseudonaja textilis) yielded trends in venom enzymatic activity that suggest seasonal upregulation of certain enzyme classes (Williams,1992). More recent studies have leveraged high resolution forms of analysis-namely chromatographic separation and mass spectrometry—and found that long-term alteration of rattlesnake diets (Gibbs et al. 2011) and long-term captivity (Freitas-de-Sousa et al., 2015) did induce modest changes in protein composition. Confounding effects of captivity stress, growth in captivity, diet, and season exist in each of the aforementioned studies, often paired with a lack of replication due to the use of pooled venom samples. Rigorous experimental procedures with before-after sampling design and statistical evaluations of resulting variation are required if we wish to gain a consensus understanding of the presence and magnitude of adult phenotypic plasticity in venom, and begin to pinpoint its proximate physiological causes.

On a practical level, it is important to identify any external factors that induce changes in snake venom and to quantify their specific impacts on protein concentrations. Venom researchers and antivenom producers often work with wild snakes that are subjected to a variety of novel, potentially stressful situations such as shaking and vibrations from transport in vehicles and temporary captivity before venom collection. The duration and exposure to these events differs within and among venom studies, 
potentially causing different states of physiological stress in the focal animals. Stressors are known to induce phenotypic plasticity in many vertebrate species, evidenced dramatically in smoltification of anadramous fishes in the transition from fresh to saltwater and in patterns of development and metamorphosis of amphibian larvae (reviewed in Wada 2008). Rattlesnakes show elevated levels of the stress hormone corticosterone (CORT) in response to transport in buckets (Holding et al. 2014a), and CORT is a known regulator of protein synthesis and activity in other animals (Duan et al. 2014; Lorenz et al. 2009; Shukla et al. 2015), suggesting that stress could affect the venom phenotype during venom re-synthesis and meriting investigation of CORT as a possible proximate mediator to expression changes in adult snake venoms.

To explore these possibilities, we quantified the extent of phenotypic plasticity in venom composition in the Northern Pacific rattlesnake (Crotalus oreganus) in a controlled experiment altering the captive conditions experienced prior to venom extraction. By subjecting wild-caught snakes to captivity, repeated venom extraction, and systematic vibratory treatments, we conducted an experiment that evaluated the extent to which venoms are affected by potential researcher-induced stressors experienced by snakes in both basic venom research and in antivenom production. Additionally, we measured plasma concentrations of CORT to assess the stressfulness of our experimental treatment and to assess potential associations between CORT levels and the changes observed in venom between successive extractions. We hypothesized that snakes venoms would be affected by novel, unpredictable situations, such as disturbance in the laboratory and predicted that snakes experiencing vibration treatments would have 
elevated CORT and altered venom composition, while control snakes would show no change in baseline CORT or venom composition.

\section{MATERIALS AND METHODS}

\section{Study animal}

We used nine adult $C$. oreganus ( 5 male, 4 female) from Montaña de Oro State

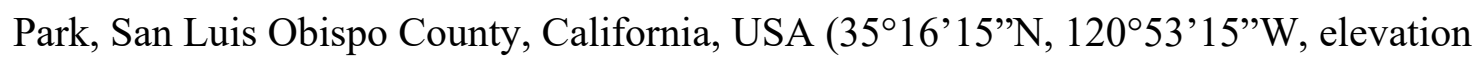
$45 \mathrm{~m}$ ), which were previously subjects of an unrelated radio-telemetry study. Snakes were housed individually in terraria with water and a heating pad on a $12 \mathrm{~L} / 12 \mathrm{D}$ cycle for at least 10 days prior to treatment after recovering from radio transmitter removal surgery. All snakes were offered a mouse two days after accession into the study, and all but one male treatment snake ate. We ran all analyses with and without the snake that did not eat, the results did not differ, and we report results from the full dataset. The California Polytechnic State University Institutional Animal Care and Use Committee (protocol \#1416), the California Department of Fish and Wildlife (scientific Collecting Permit \# SC-12963), and the California State Parks approved all procedures used. We released snakes at their original capture sites following the experiment.

\section{Researcher-induced disturbance}

Snakes were haphazardly divided into experimental groups by sex and size such that at least one size-matched pair of the same sex was available for analysis between groups. The treatment group consisted of three males and two females, and the control group consisted of two males and one female.

We aimed to create a novel stressor that mimicked the conditions a snake may experience when transported on unpaved roads before venom extraction, by creating a 
device that induced shaking and vibrations of the immediate captive environment. We attached a Sonic Bomb Alarm Clock with Super Shaker unit (Sonic Alert, Troy, MI, USA) to a wooden spine with wooden ribs in a $\mathrm{T}$ shape fitted with plastic Command hooks (3M, St. Paul, MN, USA, Figure 1). We suspended the vibrator-device with thick nylon string so each treatment cage had a row of plastic hooks resting against the back of the cage. Upon activation, the hooks knocked vigorously against the back of the cage, causing it to shake and vibrate and create a loud noise (see Supplemental Video, which demonstrates how the device agitates the snakes, as evidenced by the snakes' rattling). Vibrations began December 11, 2014 and occurred at least twice over each 24-hour period for 15 consecutive days for 20-50 minutes at a time, with duration and schedule changed haphazardly every 2-3 days in an attempt to prevent acclimation or habituation. Treatment ended December 26, 2014. Control snakes were housed in an adjacent room to isolate them from vibrations. Snakes were only handled during the treatment period for venom and blood sampling and to remove waste.

\section{Venom collection and analysis}

We had not extracted venom from these snakes prior to the current study. After 10-15 days of acclimation to the lab and before activation of the vibrator-device, an initial "before-treatment" sample of venom was collected. Venom stores were depleted during the first sampling to induce regeneration during the 15 days of treatment, which is the amount of time reported for complete replenishment of venom proteins (Currier 2012). After treatment, the final venom samples were collected. Venom was collected via a "hands off the head" technique similar to that described in Hogan (2015). Immediately after sampling, venom was stored in a $-80^{\circ} \mathrm{C}$ freezer until analysis. 
We analyzed the composition of each venom sample with reversed-phase highperformance liquid chromatography (RP-HPLC) on a Beckman System Gold HPLC (Beckman Coulter, Fullerton, CA) and used Beckman 32 Karat Software v.8.0 for peak abundance quantification. Venom sample preparation and running conditions were identical to those of Margres et al. (2014). We identified 26 separate RP-HPLC peaks in the samples, and quantified the area under each peak relative to the total area of all identified peaks, which is a useful proxy for the relative amount of protein in a specific peak by weight (Gibbs et al. 2009). To overcome the constant sum constraint of these compositional data, we used the robCompositions package (Templ et al. 2011) in $R$ ( $R$ development Core Team) to transform the data prior to statistical analysis. Specifically, we subjected the data to the isometric log-ratio (ilr) transformation, which takes the data from the simplex to real space and removes the sum constraint at the cost of one dimension of the dataset. Some of the peaks had zero values during quantification, so these were treated as trace values $(+0.01 \%)$ to permit ilr-transformation, which requires all value to be non-zero (Aitchison 1986).

We used a non-parametric MANOVA procedure, as applied by Margres et al. (2015), to compare multivariate venom composition in control and treatment snakes before and after the application of the vibration stressor. Briefly, we used the adonis function in the vegan package (Oksanen et al. 2013) in R with Euclidean distances, and ran 256 permutations (maximum number possible given our sample size) for our calculation of $\mathrm{p}$-values in the MANOVA analysis. Our model of multivariate venom composition included main effects of treatment (stress or control), time (pre-treatment or post-treatment sampling period), and a time by treatment interaction. 
To evaluate a possible relationship between baseline CORT and venom composition independent of treatment group, we summarized multivariate venom variation with the non-parametric "robust" principal components analysis (PCA) for compositional data of the robCompositions package in R (Filzmoser et al. 2009). This PCA included initial and final samples from the focal individuals of the current study, as well as 137 other $C$. oreganus venom profiles measured in the same manner (M. Holding, unpublished data), which allowed a robust estimate of components describing venom variation in these snakes. We obtained the first and second principle component (PC 1 and PC 2) scores from this analysis and determined the change in these scores for each individual between the consecutive venom extractions. We then ran a regression of individual baseline CORT (averaged from the initial, 7- and 15-day samples) against change in PC1 and change in PC 2 scores to determine whether CORT concentrations during venom regeneration are associated with the observed changes in venom composition, regardless of treatment group.

\section{Blood sampling and hormone analysis}

To evaluate the effect of vibration treatment on snake stress hormone levels, blood samples were collected from snakes to quantify levels of CORT, the major glucocorticoid hormone in reptiles (Moore, Lemaster \& Mason, 2000). A $0.5 \mathrm{ml}$ sample of blood was collected via puncture of the caudal vessels a few hours prior to treatment and at 7 and 15 days post-treatment. Time from beginning of handling to blood drawn (time-to-bleed) was recorded. Samples were collected between 09:30 and 11:48 AM to account for diel CORT changes (Lutterschmidt \& Mason 2010). The earliest sampling on day 7 occurred 12 hours after the most recent vibration bout and 3 hours after vibrations 
ended on day 15. Plasma was separated from whole blood via centrifugation at 10,000 rpm for 3 minutes and stored at $-20^{\circ} \mathrm{C}$ until radioimmunoassay.

CORT levels were determined via radioimmunoassay of plasma as described in Lind et al. (2010). Briefly, samples were extracted in dichloromethane, then dried in a $40^{\circ} \mathrm{C}$ water bath under nitrogen gas. Samples were incubated overnight in $100 \mu \mathrm{l}$ of antiserum (Esoterix Endocrinology, Calabasas Hills, CA) and 100 $\mu$ l of tritiated steroid. Unbound steroid was separated from bound steroid using dextran-coated charcoal. A liquid scintillation counter was used to count bound steroid in samples and final concentrations were corrected for extraction efficiency. Mean recovery for CORT was 78.6\%. Serial dilutions for the standard curve was performed in triplicate (CORT curve range $=2000-4 \mathrm{pg}$ ). The intra-assay coefficient of variation $(\mathrm{CV})$ was $20 \%$.

Repeated measures ANOVA was conducted in JMP Pro (12.1.0 SAS Institute) to assess effects of treatment on CORT levels. CORT and time-to-bleed variables were logtransformed to meet assumptions of normality and equal variances. Sex and time of day were not included in the final stress model, as their inclusion was non-significant.

\section{RESULTS}

\section{Vibration treatment and venom}

The vibration treatment had no detectable impact on the venom composition of the rattlesnakes. Visually, the RP-HPLC profiles of venom during the first and second venom extractions were nearly indistinguishable (Figure 2), for all control and treatment snakes. We confirmed this visual similarity with our non-parametric permutational MANOVA comparing the multivariate venom phenotype between groups (Figure 3). A significant effect of time would indicate general effects of captivity and venom extraction 
stress on venom composition, while a time by treatment interaction would indicate that vibration treatment leads to variation in venom as it is replenished after the first venom extraction. The MANOVA showed no effects of vibration treatment $\left(\mathrm{F}_{1,12}=2.19, \mathrm{P}=\right.$ $0.41)$, no impact of time $\left(\mathrm{F}_{1,12}=0.19, \mathrm{P}=0.45\right)$, and no evidence for an interaction between treatment and time $\left(\mathrm{F}_{1,12}=0.16, \mathrm{P}=0.40\right.$; Figure 2$)$, and as a result we did not attempt any post-hoc tests for differences in individual peak abundances.

\section{Vibration treatment and CORT}

Qualitatively, we observed that treatment snakes rattled upon vibrator activation (see Supplemental Video) and assumed defensive posture and rattled when an observer entered the room. These behaviors were not quantified, but we observed that control snakes did not react to researchers entering the room, and did not react to vibrator activation in the adjacent room. However, CORT levels did not appear to be affected by treatment. Initial CORT levels were similar between the treatment and control groups $\left(\mathrm{T}_{5.93}=-0.31, \mathrm{p}=0.77\right)$, and CORT was not significantly affected by the time-to-bleed $\left(\mathrm{F}_{2,6}=0.42, \mathrm{p}=0.53\right)$, by the treatment $\left(\mathrm{F}_{2,6}=2.52, \mathrm{p}=0.13\right)$, or by time $\left(\mathrm{F}_{2,6}=0.48, \mathrm{p}=0.63\right)$, and there was no detectable interaction between treatment group and time $\left(\mathrm{F}_{2,6}=0.18\right.$, $\mathrm{p}=0.84$ ). Time-to-bleed values ranged from one to seven minutes. CORT varied greatly among individuals in both groups across time, ranging from 16 to $110 \mathrm{ng} / \mathrm{ml}$ in the treatment group and 32 to $130 \mathrm{ng} / \mathrm{ml}$ in the control group (Figure 4). The most reliable predictor of CORT was the snake from which the sample was taken, as snake individual explained $69.3 \%$ in the rank order of CORT concentrations between sampling periods $\left(\mathrm{F}_{7,16}=5.2 ; \mathrm{p}<0.01\right)$ 


\section{CORT is associated with venom compositional change}

Although the changes in venom composition between the pre-treatment and posttreatment extractions appear minor and did not occur in a consistent way across time, plasma CORT concentration was associated with measured change in venom composition. Venom principle component 1 explained $26.3 \%$ of range-wide venom variation in C. oreganus, and the change in this venom PC 1 score between the two extractions showed a moderately strong, negative association with average CORT concentration in the snake $\left(\mathrm{R}^{2}=63.7 ; \mathrm{P}=0.02\right.$; Figure 5). Corticosterone concentration was not associated with the change in PC 2 scores $(\mathrm{P}=0.41)$ and we did not analyze PC 2 further.

The venom peaks with the heaviest loadings on venom PC 1 scores were peak 7 $\left(\right.$ Phospholipase A $2\left(\mathrm{PLA}_{2}\right)$; loading = 0.25), peak $8\left(\mathrm{PLA}_{2} ; 0.37\right)$, peak $11\left(\mathrm{PLA}_{2} ; 0.31\right)$, peak 12 (PLA2; 0.63), peak 20 (Snake venom serine protease (SVSP); -0.22), peak 31 (Snake venom metalloprotease (SVMP); -0.24), and peak 33 (SVMP; -0.25), Positive loadings indicate an increase as PC1 score increases, such that a negative correlation between PC1 score and CORT predicts decreases in the positively loaded proteins (PLA2)and increases in negatively loaded proteins (SVSP, SVMP) as CORT increases. The change in mean abundance in any of these individual peaks was not significantly correlated with CORT concentration $(\mathrm{P}>0.05$ for all cases, peak identification by M.L.H., unpublished data). Power was likely an issue in identifying specific peaks whose change was correlated with CORT, but the multivariate response to CORT in PC 1 was clear. 


\section{DISCUSSION}

Our study provides compelling evidence that venom composition does not change in a concerted fashion in response to disturbance in captivity during replenishment of venom stores. Specifically, we showed high repeatability of venom composition under typical laboratory conditions that is unaffected by vibration of a snake's enclosure. Repeatability of venom samples acquired through successive extractions in captive populations is a key assumption of studies that bring snakes into captivity and obtain multiple venom samples to answer a variety of biological and practical questions (Chippaux, Williams, \& White 1991; Currier et al. 2012; Salazar et al. 2009), and our work suggests that protein abundance is not grossly affected by captive conditions.

Our study used wild snakes from a single source population to investigate plasticity of venom composition, and found no effect of a potential stressor. We used newer techniques to revisit the conclusion of Gregory-Dwyer et al. (1986) that venom may not be a plastic trait in adult rattlesnakes. These authors used gel isoelectric focusing techniques to investigate plasticity of venom composition in multiple species of captive rattlesnakes exposed to seasonal fluctuations in light and temperature, with no changes observed within individuals. HPLC allowed us to investigate potential changes in relative abundance of proteins in venom which could not be visualized with iso-electric focusing, and our results also indicated that an individual's venom is repeatable. Freitas-de-Sousa et al. (2015) also used HPLC in a comparison of long-term captive and wild $B$. alternatus, and described differences they observed in pooled samples containing multiple distinct individuals as minor. Many years and possible slight geographic differences separated the B. alternatus samples, so our results add to theirs by 
experimentally altering laboratory conditions with pre- and post-treatment HPLC profiles for every individual, resulting in near-perfect similarity in paired HPLC profiles (Fig. 2). Functional and applied studies and antivenom producers using venoms from adult snakes can likely rely on repeatable samples from captive colonies (Simpson \& Jacobsen 2009; Whitaker \& Whitaker 2012), assuming diet is held constant (Gibbs et al., 2011).

As a caveat, relevant field studies have not been conducted to investigate adult venom plasticity, as all current studies have been conducted in the laboratory. The dietassociated plasticity in adult female dusky pigmy rattlesnake (Sistrurus miliarius barbouri) venom in response to a change in captive diet (Gibbs et al., 2011) suggests that venom composition of adult snakes is plastic only in response to certain cues like diet changes, a hypothesis that warrants further investigation. Laboratory settings lack the many environmental cues a snake experiences in the wild, such as fluctuating prey and water availability, changing weather patterns, and encounters with predators or conspecifics, suggesting that field manipulation studies of wild populations should be performed to investigate the possibility that relevant biological factors may lead to adult venom plasticity.

The vibration treatment did not induce measurable stress, as evidenced by the similar CORT levels between treatment and control groups. In both groups, CORT levels at all time points averaged below $100 \mathrm{ng} / \mathrm{ml}$, which is similar to baseline field levels previously reported for C. oreganus (Lind et al. 2010; Holding et al. 2014a). This is despite our observations that treatment snakes often rattled in response to activation of the shaker unit, and one treatment snake defecated after the first vibrator activation. One possibility is that the snakes were stressed by the initial vibrator activation but had 
acclimated or habituated to vibrations by mid-treatment, so baseline CORT was not affected. It is also possible the vibrator device may have acted as a multiple-exposure acute stressor (see Lutterschmidt et al. 2009; Holding et al. 2014a; Holding et al. 2014b) rather than a chronic stressor, with snakes' CORT elevating after a vibration bout and returning to baseline before blood was sampled. A recent study exposed captive garter snakes (Thamnophis elegans) to multiple-exposure acute stressors as a "chronic stress" treatment and also observed no change in baseline CORT, but observed differences in physiological responses such as wound healing and circulating metabolites in the “chronic stress" group compared to non-stressed controls (Neuman-Lee et al. 2015). Our treatment may have impacted snake physiology without sustaining elevated baseline CORT, but we still observed no change in venom composition. Taken together, venom synthesis mechanisms may be resistant to or unaffected by acute stress, assuming snakes mounted acute stress responses during treatment.

Despite a lack of concerted changes in venom or CORT concentration in response to our vibration treatment, we detected an association between averaged CORT concentrations of each snake and the small changes in venom composition that did occur. There was a negative relationship between CORT and the movement of the venom along PC 1 of venom phenotype space. This relationship was moderately strong despite our sample size of 8 snakes, suggesting a linear effect of CORT concentrations on regeneration of the venom phenotype. However, the individual proteins with high loading on PC1 were not significantly associated with CORT, possibly reflecting limited power of our small sample to detect associations at the level of single proteins. 
The proteins with high loadings on PC 1 included PLA 2 peaks that all increased and SVMP and SVSP peaks that decreased in relative abundance at higher values of PC 1. Therefore, the negative association between CORT and PC 1 suggests that higher CORT could cause a decrease in PLA2 and increase in SVMP abundance. Ontogenetic shifts in Crotaline venoms are characterized by general decreases in PLA 2 and increases in SVMP (Mackessy, 1988), as well as shifts in expression of specific proteins within the SVMP and PLA2 classes (Alape-Girón et al., 2008; Gibbs et al. 2011; Guércio et al. 2006; Saviola et al. 2015). Ontogenetic changes in venom composition are often attributed to observed shifts in diet (Alape-Girón et al. 2008; Guércio et al. 2006; Mackessy 1988), but the proximate physiological mediator of these shifts in expression is currently unknown. As CORT is implicated in many ontogenetic shifts in behavior, morphology, and physiology in vertebrates (reviewed in Wada 2008), the possibility that increased baseline CORT could direct significant changes in venom composition in snakes during maturation or under chronic stress deserves further attention. Direct manipulation of CORT levels in live snakes is possible (Lutterschmidt \& Mason, 2010), and must be done to definitively test for specific CORT-mediated changes in venom composition.

\section{CONCLUSIONS}

Our study explored the effect of researcher-induced disturbance experienced by individual snakes during venom synthesis using an experimental approach with snakes from the same source population. This study is the first to experimentally evaluate effects of short-term environmental factors on the venom phenotype in a way that is relevant to 
impacts of researchers' handling and transporting snakes. Our results suggest that snake venom synthesis mechanisms may not be affected by novel experiences such as shaking or vibrating, as is typically experienced by wild snakes when captured and transported for venom extraction. We have also shown that venom composition is highly repeatable within individual snakes after forced re-synthesis of venom in captivity, where RP-HPLC profiles taken 15 days apart are nearly indistinguishable. However, the correlation of CORT with the slight changes in venom composition that did occur raises the possibility that chronically elevated CORT may affect venom composition, and merits additional work on hormonally-mediated changes in venom protein expression. Our results provide reassuring evidence that the composition of proteins in individual rattlesnake venoms is reproducible in captive conditions and when exposed to novel disturbances, such as would occur when transporting wild snakes for venom sampling, while highlighting CORT as a target for future work on the proximate causes of venom phenotypic plasticity. 


\section{TABLE}

Table 1. Components 1 and 2 from principal component analysis of venom variation in Crotalus oreganus. The five highest loadings are bolded for component 1.

\begin{tabular}{|c|c|c|c|c|}
\hline $\begin{array}{c}\text { Principal } \\
\text { Component }\end{array}$ & Eigenvalue & $\begin{array}{c}\text { Proportion of } \\
\text { variance } \\
\text { explained }\end{array}$ & $\begin{array}{c}\text { Venom Peak } \\
\text { Number }\end{array}$ & Loading \\
\hline \multirow[t]{34}{*}{ PC 1} & \multirow[t]{34}{*}{18.30} & \multirow[t]{34}{*}{0.26} & 1 & -0.04 \\
\hline & & & 2 & -0.04 \\
\hline & & & 3 & -0.09 \\
\hline & & & 4 & -0.04 \\
\hline & & & 5 & 0.03 \\
\hline & & & 6 & -0.11 \\
\hline & & & 7 & 0.35 \\
\hline & & & 8 & 0.32 \\
\hline & & & 9 & 0.14 \\
\hline & & & 10 & 0.01 \\
\hline & & & 11 & 0.32 \\
\hline & & & 12 & 0.53 \\
\hline & & & 13 & -0.10 \\
\hline & & & 14 & 0.08 \\
\hline & & & 15 & -0.15 \\
\hline & & & 16 & 0.07 \\
\hline & & & 17 & 0.008 \\
\hline & & & 18 & -0.19 \\
\hline & & & 19 & -0.19 \\
\hline & & & 20 & -0.24 \\
\hline & & & 21 & -0.02 \\
\hline & & & 22 & -0.03 \\
\hline & & & 23 & -0.05 \\
\hline & & & 24 & -0.06 \\
\hline & & & 25 & -0.10 \\
\hline & & & 26 & -0.02 \\
\hline & & & 27 & 0.16 \\
\hline & & & 28 & 0.04 \\
\hline & & & 29 & -0.17 \\
\hline & & & 30 & -0.004 \\
\hline & & & 31 & -0.29 \\
\hline & & & 32 & -0.03 \\
\hline & & & 33 & -0.02 \\
\hline & & & 34 & -0.05 \\
\hline
\end{tabular}


Table 1 cont'd

\begin{tabular}{|c|c|c|c|c|}
\hline $\begin{array}{c}\text { Principal } \\
\text { Component }\end{array}$ & Eigenvalue & $\begin{array}{c}\text { Proportion of } \\
\text { variance } \\
\text { explained }\end{array}$ & $\begin{array}{c}\text { Venom Peak } \\
\text { Number }\end{array}$ & Loading \\
\hline \multirow[t]{34}{*}{ PC 2} & \multirow[t]{34}{*}{12.96} & \multirow[t]{34}{*}{0.19} & 1 & 0.005 \\
\hline & & & 2 & -0.005 \\
\hline & & & 3 & -0.03 \\
\hline & & & 4 & -0.05 \\
\hline & & & 5 & -0.05 \\
\hline & & & 6 & 0.05 \\
\hline & & & 7 & 0.16 \\
\hline & & & 8 & -0.09 \\
\hline & & & 9 & 0.04 \\
\hline & & & 10 & -0.03 \\
\hline & & & 11 & -0.12 \\
\hline & & & 12 & -0.16 \\
\hline & & & 13 & -0.09 \\
\hline & & & 14 & 0.02 \\
\hline & & & 15 & -0.01 \\
\hline & & & 16 & 0.04 \\
\hline & & & 17 & -0.04 \\
\hline & & & 18 & -0.17 \\
\hline & & & 19 & -0.11 \\
\hline & & & 20 & -0.13 \\
\hline & & & 21 & -0.03 \\
\hline & & & 22 & -0.007 \\
\hline & & & 23 & 0.27 \\
\hline & & & 24 & -0.14 \\
\hline & & & 25 & -0.11 \\
\hline & & & 26 & -0.04 \\
\hline & & & 27 & -0.07 \\
\hline & & & 28 & 0.12 \\
\hline & & & 29 & -0.12 \\
\hline & & & 30 & 0.02 \\
\hline & & & 31 & 0.05 \\
\hline & & & 32 & -0.007 \\
\hline & & & 33 & 0.84 \\
\hline & & & 34 & -0.005 \\
\hline
\end{tabular}




\section{FIGURES}

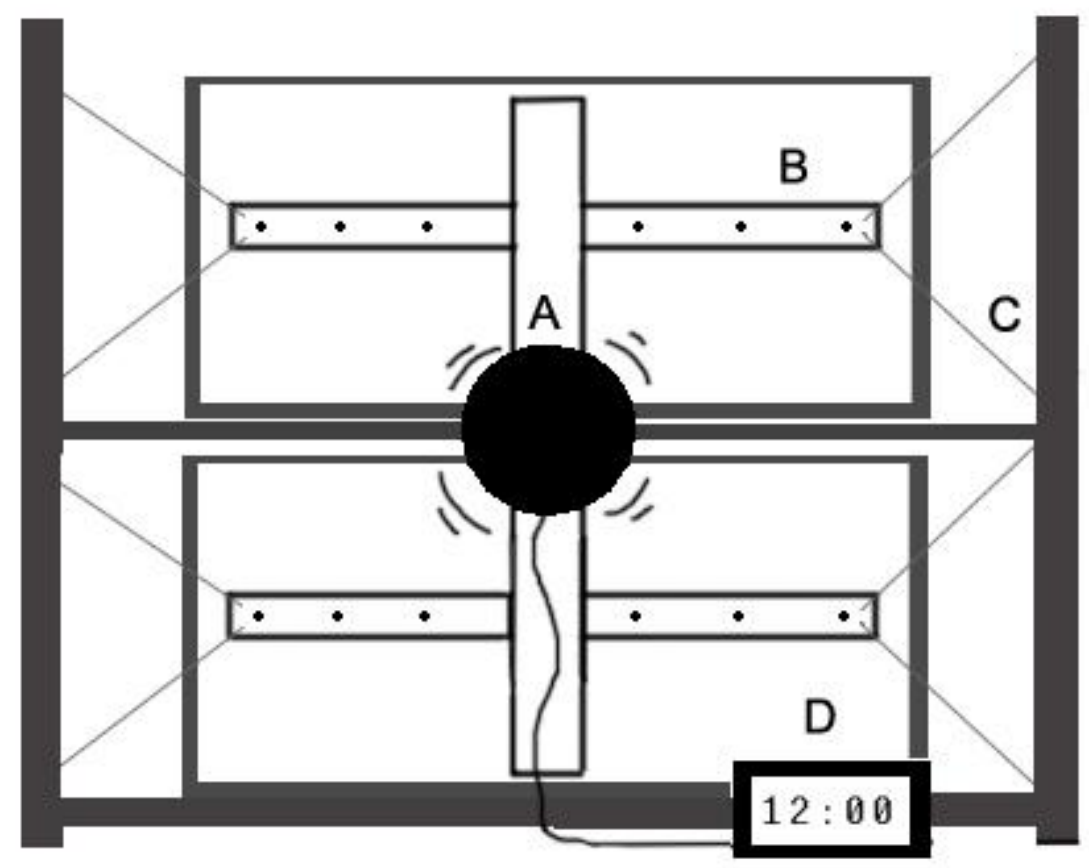

Figure 1. Example vibrator device setup viewed from the back of the cages. Sonic Boom vibrator unit (A) is attached to the wooden ribs, which are fitted with plastic hooks that rest upon the back of the cage (B). The contraption is suspended via nylon string attached to the metal shelving unit (C), and its vibrations are activated and controlled by the Alarm clock timer (D). 

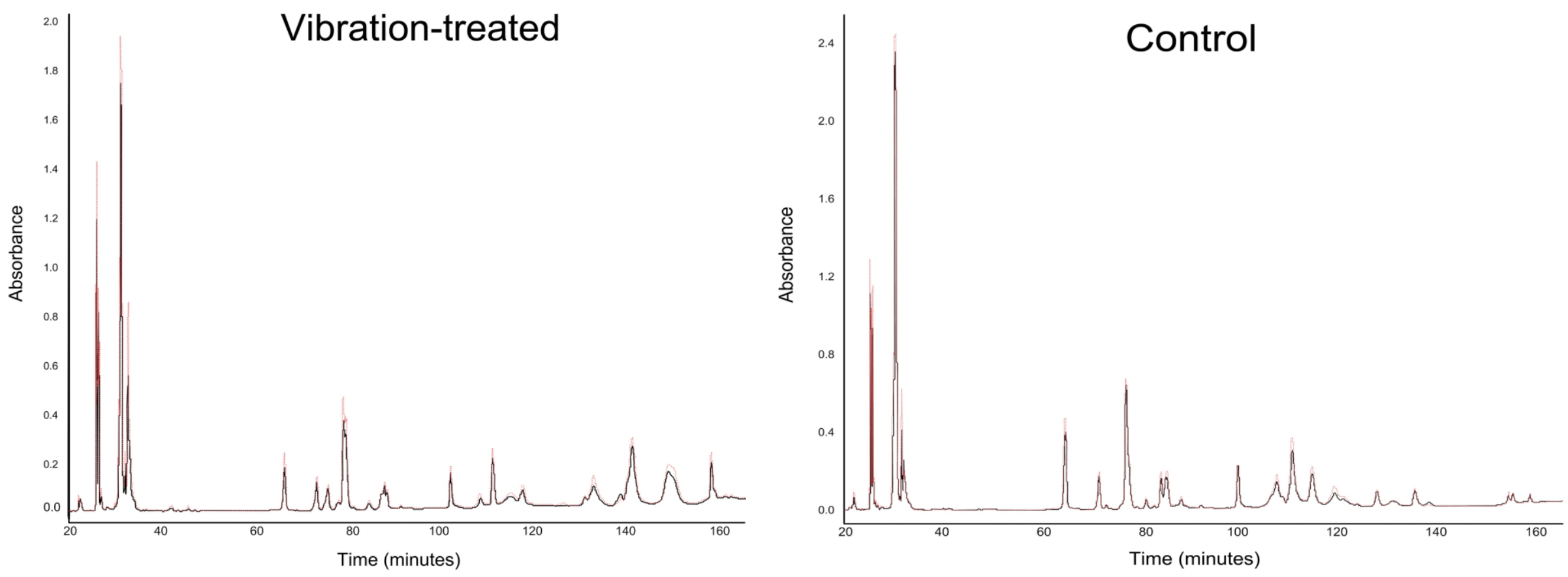

Figure 2. Representative HPLC chromatograms, showing venom profiles of control Crotalus oreganus subjected to captivity only (right) and a treatment snake subjected to vibration treatments (left) between the two venom extraction time points (pre-treatment $=$ black, post $=$ dotted red). Relative abundance of venom proteins are represented by individual peaks. The profiles overlap in a near-perfect fashion, indicating very little change in the relative abundance of the venom proteins within individuals' venoms over time, and no effect of vibration treatment. 
Vibration-treated

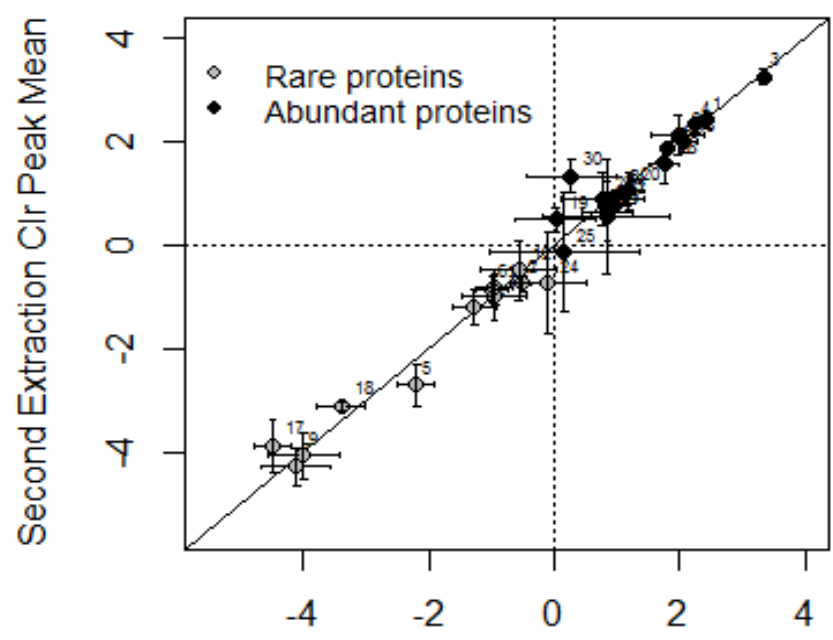

First Extraction Clr Peak Mean
Control

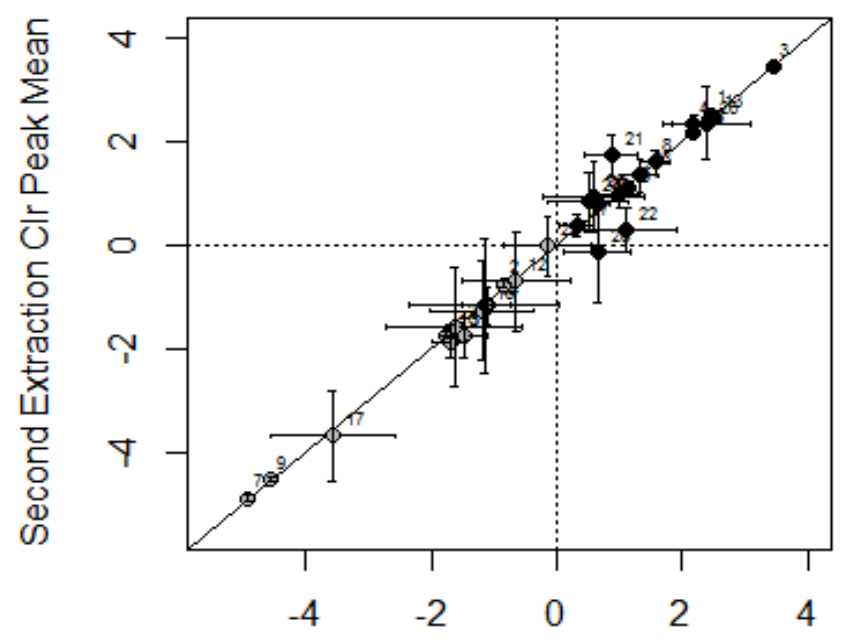

First Extraction Clr Peak Mean

Figure 3. Individual mean centered log-ratio transformed abundance for each RP-HPLC peak across vibration-treated and control Crotalus oreganus. The position on the $\mathrm{x}$-axis of each graph indicates the peak mean abundance during the first extraction of venom, and the position on the $\mathrm{y}$-axis indicates that for the second extraction (post-treatment). High-expression proteins are represented by black circles, low-expression proteins by grey. Bars indicate one S.E.M., the solid line shows perfect agreement in peak relative abundance between initial and final sampling, and the dashed lines indicates the geometric mean of the dataset. Proteins with clr peak means below the geometric mean in both groups were classified as low expression proteins following Margres et al. (2016). 


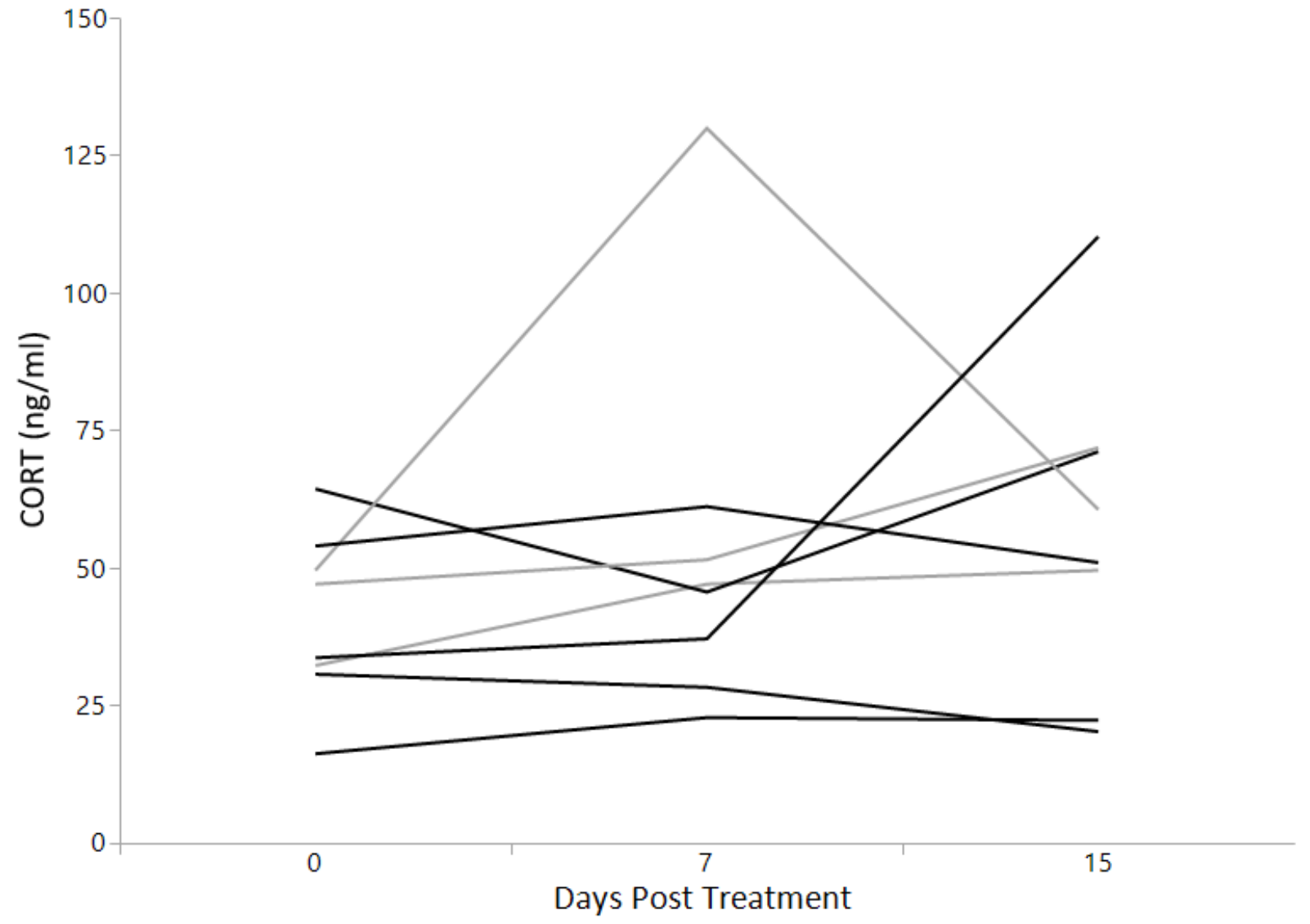

Figure 4. Plasma corticosterone (CORT) values of individual Crotalus oreganus at initial, 7 days post, and 15 days post vibration treatment. Black lines represent treatment snakes, gray lines control snakes. There was no effect of treatment or time on CORT levels $\left(F_{2,6}=2.52, p=0.13\right.$; $\left.\mathrm{F}_{2,6}=0.48, \mathrm{p}=0.63\right)$. 


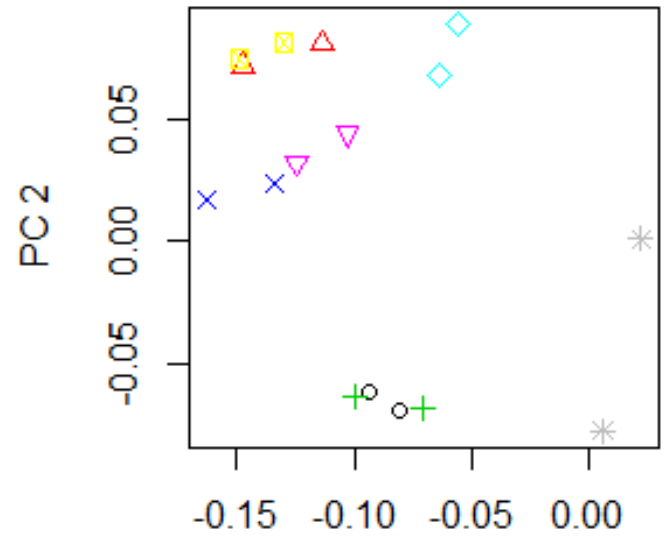

PC 1

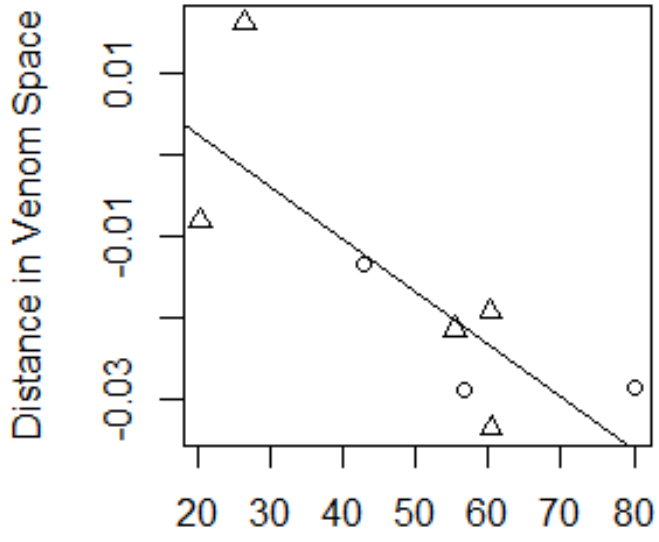

Average Corticosterone $(\mathrm{ng} / \mathrm{mL})$

Figure 5. Association between movement through venom phenotype space and CORT for individual Crotalus oreganus. Biplot (left) of venom principle component 1 and 2, where the first and second venom samples from each snake are coded by the same symbol. Scatterplot (right) showing the association between the change in venom principle component 1 scores during captivity and the average baseline CORT concentration of individual control (circles) and vibration-treated (triangles) snakes $\left(\mathrm{R}^{2}=63.7 ; \mathrm{p}=0.02\right)$. 


\section{CHAPTER II: EFFECTS OF CORTICOSTERONE IMPLANTS ON CROTALUS HELLERI VENOM TOTAL PROTEIN CONCENTRATION AND ACTIVITY}

\section{INTRODUCTION}

Natural variation in snake venom composition could potentially carry large biological and biomedical consequences. For example, variation in venom composition could lead to changes in prey capture success or digestion (Mackessy 1988; Gibbs \& Mackessy 2009), variable symptoms from snake envenomation in humans (Bush et al. 2002; Wasserberger et al. 2006; Calvete et al. 2011; Massey et al. 2012; Casewell et al. 2014), and differences in efficacy of antivenom treatment on snakebites from a single species (Calvete et al. 2009; Casewell et al. 2014; Núñez et al. 2009; Saviola et al. 2015). The most widely documented instances of venom plasticity occur as ontogenetic shifts in crotaline venom protein composition, which is marked by a shift from neurotoxic to myotoxic and proteolytic as snakes grow larger (Alape-Girón et al. 2008; Mackessy et al. 1988; Saviola et al. 2015). Venoms also exhibit shifts in expression of specific proteins within the snake venom metalloproteinase (SVMP) and phospholipase $\mathrm{A}_{2}$ (PLA2) classes (Alape-Girón et al., 2008; Gibbs et al. 2011; Guércio et al. 2006; Saviola et al. 2015), which are considered adaptive in that they often mirror size-associated changes in diet (Mackessy 1996 ; Alape-Girón et al., 2008; Guércio et al., 2006; Mackessy, 1988; Gibbs et al. 2011). Plasticity within adult venoms has been documented in response to a change in diet (Gibbs et al. 2011), season (Gubensek et al. 1974), and repeated sampling (Williams 1992). However, the proximal physiological mediators of ontogenetic shifts or of variation within adult snake venom protein composition and expression are currently unknown. 
One candidate is the glucocorticoid hormone, corticosterone (CORT), the main circulating stress hormone in reptiles (Moore, Lemaster \& Mason 2000). Across several vertebrate groups, CORT is implicated in ontogenetic shifts in behavior, morphology, and physiology (reviewed in Wada 2008), such as smolitification of anadramous fishes and metamorphosis of amphibian larvae. At high levels, CORT is known to decrease or inhibit protein synthesis by affecting transcription factor recruitment (Sapolsky, Romero \& Munck 2000, Romero et al. 2005; Duan et al. 2014; DesRochers et al. 2009) and to influence enzyme activity though upstream actions on protein kinase activation pathways (Kang et al. 2010; Li et al. 2001; Harms et al. 2007; Liu et al. 2010). Snake venoms exhibit a number of proteins and toxins homologous to those found in other vertebrates, such as matrix metalloproteases and PLA2 (Kini 2003; Fry 2005; Fry \& Wüster 2004). Homologs of venom metalloproteases and PLA 2 enzymes are negatively affected by CORT, which causes decreases in both activity and abundance (Chuang et al. 2015; Shikatani et al. 2012; Marqueti et al. 2006; Lorenz et al. 2009; Vishwanath et al. 1993). Recently, CORT was implicated as an in vitro high-affinity inhibitor of PLA2 type II in Russell's viper venom (Shukla et al. 2015). Despite strong evidence for CORT-regulation in homologous proteins, CORT has not been investigated as a possible proximate mediator of snake venom activity and synthesis in vivo.

CORT levels across many vertebrate species vary ontogenetically (Wada 2008), by season (Moore, Greene \& Mason 2001; Holding et al. 2012b; Lind et al. 2010), reproductive state (Berger et al. 2005; Cree, Amey \& Whittier 2000; Schuett \& Grober 2000; Lutterschmidt et al. 2009), and increase in response to chronic and acute stressors (Berger et al. 2005; Hopkins \& DuRant 2011; Lutterschmidt et al. 2009; Baugh et al. 
2014). During prolonged periods of chronic stress, such as drought or extensive fasting, glucocorticoids are implicated in directing organism survival, triggering an emergency life history stage to mobilize energy stores and downregulate nonessential processes, such as reproduction (Wingfield et al. 1998; Busch \& Hayward 2009). Prey availability for reptilian predators depends largely on the surrounding environmental conditions (Jessop, Woodford \& Symonds 2013; Sperry \& Weatherhead 2008). Rattlesnakes are known to survive prolonged drought conditions with low prey availability (McCue 2007), but next to nothing is known about how their most important feeding tool, venom, is affected by these periods of chronic stress, and whether this influences predatory success. If venom is affected by chronic stress, or high levels of CORT, this may also affect efficacy of antivenom in treating snakebite (Calvete et al. 2009; Casewell et al. 2014; Núñez et al. 2009; Saviola et al. 2015). From both an ecological and biomedical perspective, it is important to understand how snake venom is affected by periods of elevated CORT.

To explore the possibility that CORT affects venom synthesis, composition, and activity, we conducted a repeated-measures study with radio-telemetered Southern Pacific rattlesnakes (Crotalus helleri). Individual snakes were either treated with CORT implants of with blank implants and their venoms were compared using a before-after treatment study design. Venom proteins with known homologs affected by CORT were assessed. Additionally, plasma CORT levels were quantified to assess the effectiveness of the implants, and effects of baseline CORT on venom activity. If CORT directs significant changes in protein activity and concentration in snakes under chronic stress, we predicted that rattlesnakes implanted with CORT would exhibit lower activity in 
venom proteins PLA 2 and SVMP, as well as decreased overall protein concentration relative to controls.

\section{METHODS}

\section{Study animal and site}

Thirty adult male $C$. helleri were captured through visual encounter surveys at the University of California at Santa Barbara's Sedgwick Reserve in Santa Ynez, Santa Barbara County, California ( $34.6928^{\circ} \mathrm{N}, 120.0406^{\circ} \mathrm{W}$, elevation: $\left.290 \mathrm{~m}\right)$ from mid-April to early May 2015. Snakes received intra-muscular passive integrated transponder tags (MUSICC Chip, AVID Identification Systems, Inc, Norco, CA, USA), and the three basal rattle segments were filled with non-toxic acrylic paint to a predetermined color code for future identification. The snakes at the site were found mainly in cattle-disturbed valley oak savannah habitat, with areas of coastal sage scrub habitat. Rattlesnakes were collected under California Department of Fish and Wildlife Scientific Collecting Permit \# SC-13134, and experimental procedures were approved by the California Polytechnic State University Institutional Animal Care and Use Committee (Protocol \#1416) and the University of California at Santa Barbara Institutional Animal Care and Use Committee (Protocol \#Taylor 1415, Animal Activity \#027).

Snakes were transported to California Polytechnic State University for radiotransmitter and thermal data logger implantation. Snakes were housed individually in 30 " x 12" x 12" Visionarium cages (Vision Products) with a heat pad, hide box, and water ad libitum. Snakes were anesthestized via isofluorane inhalation (Vet One, MWI, 
USA) and received intra-coelomic implants of $3.25 \mathrm{~g}$ temperature data logger (Thermochron iButton model DS1922L\#F50, Maxim Integrated, San Jose, California) and a radiotransmitter weighing 5.3, 11, or $13.5 \mathrm{~g}$ (Holohil Systems Ltd., Carp, Ontario, Canada). Combined weights of implants totaled less than $5 \%$ of snake total body mass. Snakes were allowed to recover for 1-2 days before release at the site of capture.

\section{Experimental administration of exogenous corticosterone}

Snakes were allowed to recover in the field for at least two weeks post-surgery prior to experimental manipulation in late May 2015. Median mass of snakes was $800 \mathrm{~g}$; thus we divided the snakes into two groups (size classes) above and below this value. Snakes greater than $800 \mathrm{~g}$ were given two $15 \mathrm{~mm}$ (large) implants, and snakes less than $800 \mathrm{~g}$ received two $7.5 \mathrm{~mm}$ (small) implants. Snakes within each weight class were randomly assigned to either a treatment group or control group. Treatment snakes received implants filled with CORT, while control snakes received blank implants. Seven large snakes and eight small snakes were assigned to the CORT treatment group. Eight large and seven small snakes were assigned to the control group.

Corticosterone implants were made by plugging one end of a $15 \mathrm{~mm}$ or $7.5 \mathrm{~mm}$ silastic diffusion tubing (Dow Corning, Clarkesville, TN, USA: $1.47 \mathrm{~mm}$ inner, $1.96 \mathrm{~mm}$ outer diameter) with $2 \mathrm{~mm}$ silicone caulking (Momentive Performance Materials Inc, Huntersville, NC), which dried for 24 hours. The tubing was then filled with crystalline CORT (Sigma C2505-500 mg Lot\# SLBJ5337V), and plugged with 2mm silicone and allowed to dry for 24 hours. Control implants were left empty, then plugged with silicone. 
For each size class, triple the amount of implants required were created. Implants were weighed, with the upper and lower third of implants discarded to improve dosing accuracy. $15 \mathrm{~mm}$ treatment implants averaged $6.1 \mathrm{mg}$ CORT, while $7.5 \mathrm{~mm}$ treatment implants averaged $3.6 \mathrm{mg}$ CORT.

Implants were soaked in $0.9 \%$ saline solution for 12 hours prior to implantation to facilitate CORT release. Each snake received two implants. The first implant was designed as fast-release by poking one small hole in the tubing with an insulin syringe. The second implant was left intact as a slow-release dose. Implants were injected intracoelomically on each side of the lower third of the body via a sterilized $12 \mathrm{~g}$ PIT tag injector. Maximum total dosage of large CORT-treated snakes averaged 11.91+/-1.94 $\mathrm{mg} / \mathrm{kg}$, while small CORT-treated snakes ranged $14.71+/-4.23 \mathrm{mg} / \mathrm{kg}$.

\section{Sampling period - Capture data}

Each snake was sampled for blood and venom initially on the day of implantation immediately before implants were administered (pre-implant sample), then approximately 15 days (sample 2) and 30 days post-implant (sample 3).

Snakes were captured using tongs (Whitco Manufacturing Inc., Weatherford TX, USA ) and tubed for safe restraint (Plastic Restraining Tubes, Midwest Tongs, Greenwood, MO, USA). Only snakes above ground or visible in a burrow were captured to avoid disturbing habitat and inducing unequal stress-responses across individuals. A few snakes were unavailable for capture during the sampling period, as they were deep underground in burrow complexes. These snakes were sampled at the next possible opportunity, and then sampled within a ten to fifteen day period thereafter to maintain 
consistent venom regeneration time across individuals. Snakes were excavated from burrows if the process was estimated to take less than ten minutes from start of digging to blood sample collection. Two snakes were unavailable for sampling, and one snake died of an injury likely caused by predation. This resulted in seven small and seven large snakes in the treatment group, and eight small and five large snakes in the control group. One large control snake only had an initial sample, but its data were used in some parts of the analysis.

Snakes were recaptured in October and November 2015 for radiotransmitter and data logger removal and released at their sites of capture after recovery.

\section{Blood}

Time from beginning of handling (or disturbance of habitat) to blood drawn (time-to-bleed) was recorded. A $0.5 \mathrm{ml}$ sample of blood was collected via puncture of the caudal vessels with a 25 gauge $3 / 4$ " syringe at each capture. Within 48 hours, plasma was separated from whole blood via centrifugation at $10,000 \mathrm{rpm}$ for three minutes and stored at $-20^{\circ} \mathrm{C}$ until radioimmunoassay (see below). Data points with unusually high baseline CORT (>100 ng/mL) were excluded from analysis as outliers. Time of day was recorded to account for variation due to diel CORT cycling (Lutterschmidt \& Mason 2010).

\section{Radioimmunoassay of blood plasma}

CORT levels were determined via radioimmunoassay of plasma as described in Lind et al. (2010). Briefly, samples were extracted in dichloromethane, then dried in a $40^{\circ} \mathrm{C}$ water bath under nitrogen gas. Samples were incubated overnight in $100 \mu \mathrm{l}$ of antiserum (Esoterix Endocrinology, Calabasas Hills, CA) and $100 \mu 1$ of tritiated steroid. 
Unbound steroid was separated from bound steroid using dextran-coated charcoal. A liquid scintillation counter was used to count bound steroid in samples, and final concentrations were corrected for extraction efficiency. Mean recovery for CORT was 64\%. Serial dilutions for the standard curve was performed in triplicate (CORT curve range $=2000-4 \mathrm{pg}$ ). The limit of detection was $2.5 \mathrm{ng} / \mathrm{ml}$, and intra-assay coefficient of variation $(\mathrm{CV})$ was $7.3 \%$.

\section{Venom}

Venom stores were depleted at the each sampling to induce regeneration over approximately 15 days, which is the amount of time reported for complete replenishment of venom proteins (Currier 2012). Venom was collected via a "hands off the head" technique similar to that described in Hogan (2015). Venom was drawn from each snake by coaxing its head out of the end of the tube, gently gripping the neck with forceps, and inducing a bite onto a parafilm-wrapped beaker. Once the snake bit, rubber dipped tongs were used to massage venom glands by squeezing and massaging in a rolling motion from back to front. Two beakers were used to attempt to extract all venom from the snake, but most snakes appeared to yield all venom in the first attempt. Venom was immediately flash frozen and stored on dry ice, then transferred to a $-80^{\circ} \mathrm{C}$ freezer until analysis.

Some snakes expended venom on tongs or tubes during capture, so not all venom expended is represented in the sample. However, the goal was to extract all venom from the snake to initiate venom regeneration under the treatment protocols, so even with venom lost on tongs, this was still achieved. It is unlikely that the venom lost to the tongs represents a different composition than that expended into the beaker. Snakes with a 
recent meal (determined via palpation) gave visibly smaller venom samples, likely having spent venom recently on the meal. However, food bulges were only observed in snakes during the pre-implant sampling (e.g., meals are unlikely to impact the interpretation of the CORT implants). After venom was drawn, the snakes were released at the site of capture.

The initial venom sample from one small treatment snake was too small to quantify, and one large control snake had only an initial sample, so these snakes were excluded from venom analysis due to the repeated measures design.

\section{Venom protein concentration}

Venom was sent to Ohio State University for analysis. We determined the protein concentration of each venom sample using the microassay protocol of the Quick Start Bradford Protein Assay Kit (Bio-Rad Laboratories, Hercules, CA, USA) with a bovine serum albumen standard. Whole venom was diluted 1:200 in phosphate-buffered saline prior to determining the protein concentration.

\section{Venom activity levels}

We determined the phospholipase $\mathrm{A}_{2}\left(\mathrm{PLA}_{2}\right)$ and snake venom metalloproteinase (SVMP) activity of each venom sample as measures of venom function. We measured SVMP activity of each venom sample using the standard product protocol in the EnzChek Gelatinase/Collagenase Kit (Life Technologies, Carlsbad, CA, USA), as this kit has been previously shown to measure metalloproteinase activity of rattlesnake venoms (Biardi et al. 2011). The fluorescently-labelled gelatin substrate was diluted to a concentration of 1:50, and $0.5 \mathrm{ug}$ of venom diluted in PBS was placed in each reaction 
well on a 96-well microplate (Corning \#3912, Corning, Tewksbury, MA, USA). We measured fluorescence intensity in relative fluorescence units (RFU) every 1 minute after the addition to the gelatin substrate using a FLUOstar Omega microplate reader (BMG Labtech, Ortenberg, Germany), subtracting the background fluorescence of blank (substrate only) wells from each measurement. From these data, we calculated the slope (RFU/min) from the linear part of the reaction ( 0 - 9 minutes after substrate addition), which we used as our measure of venom SVMP activity. Each venom was measured in triplicate, and the average slope of the three reactions was used during data analysis. Larger slopes indicate that the venom is degrading the substrate more quickly, which means a higher SVMP enzymatic activity of the focal sample.

Venom PLA2 activity was assayed in a similar way to SVMP activity by using the EnzChek PLA2 Assay Kit (Life Technologies, Waltham, MA, USA), following standard product protocols. Again, 0.5 ug of venom was pipetted into each well, and fluorescence intensity was measured every minute. The linear part of the reaction (1-15 minutes) was used to calculate the slope of each reaction. Samples were run in duplicate, and the average of the two reactions was used for further analysis.

\section{Statistical analysis}

All statistical tests were performed in JMP Pro 12 (SAS Institute, 2015). Variables were tested for normality and homogeneity of variances. When these assumptions were violated, transformations were applied to correct the variables.

Repeated measures ANOVA was used to determine the effect of CORT implant treatment on baseline CORT levels, venom activity, and protein concentration. Relevant 
terms were included in the model, and non-significant terms were eliminated in order of least significance. If elimination did not change the significance of other variables, the full model is reported. In cases where Treatment was the least significant variable, the full model is reported. Repeatability analyses were conducted on venom variables to determine the proportion of variation observed due to between-snake variance versus within-snake variance. This was calculated using the mean squares values from a oneway analysis of variance using Snake ID as the predictor, based on a previously established method (Lessels and Boag 1987; Narayan and Hero 2013). Repeatability analyses were used to determine whether the 'individual' predicts response in venom variables to a greater degree than the among-snake variables used in the repeated measures ANCOVA (such as SVL and treatment group).

\section{All models}

Due to the repeated measures design where the first sample from all snakes is preimplant, 'Treatment by sampling period interaction' refers to the effects associated with CORT implants over time. 'Treatment' will refer to the main effect of treatment, and 'Repeated sampling' will refer to the main effect of sampling period, or effects due to time alone.

Size class was included in all models as a main effect in the analyses, to account for the method of experimental randomization, but these results are not reported. SVL was included as a covariate to account for continuous variation due size differences. Body condition was calculated by dividing $\log ($ mass $)$ by $\log (\mathrm{SVL})$. 
Tukey's Post-hoc tests were used to determine groups that were significantly different from one another.

\section{Baseline Circulating CORT}

Time of day, time-to-bleed, and body temperature were included as covariates in the repeated measures ANCOVA. One treatment snake was excluded from CORT analysis, as only a pre-implant sample was collected, and no other samples were available from this individual for comparison. Dixon's Q test identified one outlier in the dataset with exceedingly high CORT levels at sample 3 (Dean and Dixon 1951; SAS Institute, Cary, NC 2014). This sample was excluded from the dataset for analysis.

\section{Venom}

SVMP and PLA2 activity were log10-transformed, and total protein concentration was squared to improve homogeneity of variances.

\section{RESULTS}

\section{Implants elevated baseline CORT in treatment snakes}

Compared to pre-implant treatment levels, CORT was elevated in treatment group snakes at sample $2\left(\mathrm{~F}_{2,41.35}=3.33, \mathrm{p}=0.046\right)$, and was an average of $60 \%$ higher than control snakes during the same period (Figure 6). By sample 3, CORT had returned to pre-implant levels in treatment snakes. There was no effect of repeated sampling $\left(\mathrm{F}_{2,43.3}=1.34, \mathrm{p}=0.27\right)$, but treatment had a marginal effect, meaning across all timepoints (including pre-implant), treatment snakes trended towards higher $\operatorname{CORT}\left(\mathrm{F}_{1,22.43}=3.58\right.$, 
$\mathrm{p}=0.07$ ). Tukey's post hoc tests revealed that this is likely driven by the higher CORT levels of treatment snakes at sample 2. Time to blood (Log), Time of day, Body temperature, and $\operatorname{SVL}\left({ }^{\wedge} 2\right)$ were included in the model as covariates, but were not

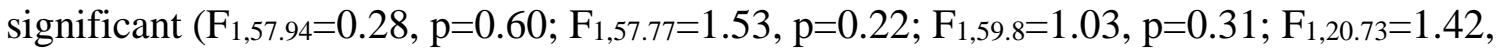
$\mathrm{p}=0.25)$.

\section{SVMP activity}

SVMP activity was affected by repeated sampling $\left(\mathrm{F}_{2,39.06}=3.98, \mathrm{p}=0.03\right)$, with all snakes averaging $400 \mathrm{RFU} / \mathrm{min}$ higher SVMP activity in sample 3 than pre-implant sample, regardless of treatment group (Figure 7A). There was no main effect of treatment

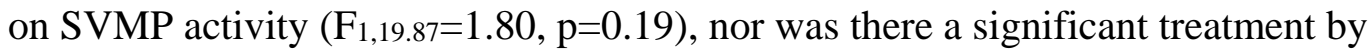
sampling period interaction $\left(\mathrm{F}_{2,39.1}=0.07, \mathrm{p}=0.93\right)$. Baseline CORT level at sampling and SVL were not significant covariates $\left(\mathrm{F}_{1,40.42}=0.65\right.$, $\mathrm{p}=0.42$, Figure $A 1 ; \mathrm{F}_{1,19.98}=3.04$, $\mathrm{p}=0.10 ;$ Figure A2).

Distribution of SVMP activity was split with snakes falling into two categories of low (252-740 RFU/min) and high (2267-5750 RFU/min) phenotypes. No snake crossed from one phenotype to the next during the sampling period.

Investigation of each phenotype group separately did not change results regarding treatment by sampling period or CORT levels (Figure A3, Figure A4). However, there was no longer an effect of repeated sampling (High: $\mathrm{F}_{1,21.89}=1.13$, $\mathrm{p}=0.34$, Figure A5; Low: $\mathrm{F}_{2,12.02}=2.65$, $\mathrm{p}=0.11$, Figure A6). SVL was a significant covariate within the high SVMP phenotype group only (High: $F_{1,10.83}=6.82$, $p=0.02$, Figure A7; Low: $F_{1,5.96}=0.29$, 
p=0.61, Figure A8), with larger High SVMP phenotype snakes having higher SVMP activity.

To account for the possibility that treatment snakes experienced a higher dose of CORT between pre-implant sample and sample 2, we ran a one-way ANCOVA investigating the change in activity over this time period. There was no effect of treatment $\left(\mathrm{F}_{1,17}=0.94, \mathrm{p}=0.35\right.$, Figure $\left.\mathrm{A} 9\right)$, or an effect of days between samples $\left(\mathrm{F}_{1,17}=0.01, \mathrm{p}=0.92\right.$, Figure A10). Change in baseline CORT was not a significant covariate $\left(F_{1,17}=0.07, p=0.80\right.$, Figure A11), and neither was $\operatorname{SVL}\left(F_{1,17}=0.06, p=0.81\right.$, Figure A12). SVMP phenotype did not appear to have an influence in change in SVMP activity $\left(\mathrm{F}_{1,17}=0.39, \mathrm{p}=0.54\right.$, Figure A13), indicating that both phenotypes respond similarly to repeated sampling, and are unaffected by treatment.

\section{$\mathrm{PLA}_{2}$ Activity}

There was no treatment by sampling period interaction $\left(\mathrm{F}_{2,39.81}=0.57, \mathrm{p}=0.57\right)$ or effect of repeated sampling $\left(\mathrm{F}_{2,39.7}=0.40, \mathrm{p}=0.67\right)$ on $\mathrm{PLA}_{2}$ activity (Figure $\left.2 \mathrm{~B}\right)$. There was no main effect of treatment $\left(\mathrm{F}_{1,21.28}=0.20, \mathrm{p}=0.66\right)$. Baseline $\mathrm{CORT}$ at sampling was a significant covariate $\left(\mathrm{F}_{1,42.9}=4.48, \mathrm{p}=0.04\right.$, Figure 8$)$, with higher baseline CORT associated with higher PLA2 activity. SVL was not a significant covariate for PLA2 activity $\left(\mathrm{F}_{1,21.48}=0.01, \mathrm{p}=0.91\right.$, Figure A14 $)$.

To account for the possibility that treatment snakes experienced a higher dose of CORT between pre-Implant and sample 2, we ran a one-way ANCOVA investigating the change in activity over this time period. No effect of treatment was found $\left(\mathrm{F}_{1,17}=0.31\right.$, $\mathrm{p}=0.59$, Figure A15. Change in baseline CORT was not a significant covariate 
$\left(F_{1,17}=0.16, p=0.69\right.$, Figure A16) SVL was not a significant covariate $\left(F_{1,17}=2.88, p=0.11\right.$, Figure A17), but initial body condition weakly negatively correlated with change in activity $\left(\mathrm{F}_{1,17}=6.42, \mathrm{p}=0.048\right.$, Figure A18) There was a strong effect of time between samples, with activity increasing as days between samples increased $\left(\mathrm{F}_{1,17}=22.58\right.$, $\mathrm{p}=0.0002$, Figure 9). This effect appeared to be driven by three snakes for which samples were $>20$ days apart. Analysis was re-run with these snakes removed, and time between samples was no longer a significant covariate $\left(\mathrm{F}_{1,14}=0.22, \mathrm{p}=0.65\right.$, Figure A19). None of the other variables reached significance in the reduced model.

\section{Total protein concentration of venom}

There was no treatment by sampling interaction $\left(\mathrm{F}_{2,44.08}=2.21, \mathrm{p}=0.12\right)$ or a main

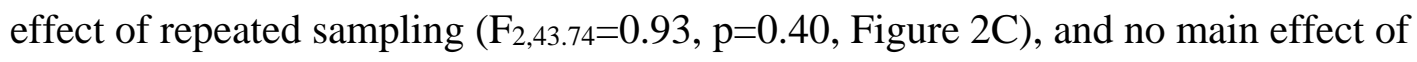
treatment $\left(\mathrm{F}_{1,19.56}=0.38, \mathrm{p}=0.54\right)$ on total protein concentration in venom. Neither SVL $\left(\mathrm{F}_{1,22.23}=0.04, \mathrm{p}=0.85\right.$, Figure A20 $)$ nor baseline CORT were significant covariates $\left(F_{1,60.97}, p=0.38, p=0.54\right.$, Figure A21) SVMP activity phenotype was a significant predictor of total protein concentration, with low phenotype SVMP snakes have lower total protein than high SVMP phenotype snakes, regardless of sampling period $\left(\mathrm{F}_{1,20.5}=12.18, \mathrm{p}=0.002\right.$, Figure 10$)$.

Treatment did not affect change in total protein concentration from pre-implant to sampling $2\left(\mathrm{~F}_{1,16}=1.72, \mathrm{p}=0.21\right.$, Figure A22). There was a nonsignificant positive trend $\left(\mathrm{F}_{1,16}=4.25, \mathrm{p}=0.056\right)$ between time between samples and total protein concentration (Figure 11). Change in baseline CORT between samplings was not a significant covariate $\left(\mathrm{F}_{1,16}=0.12, \mathrm{p}=0.73\right.$, Figure A23). Neither body condition nor $\operatorname{SVL}\left(\mathrm{F}_{1,16}=1.23, \mathrm{p}=0.73\right.$, Figure A24) were significant $\left(\mathrm{F}_{1,16}=1.35, \mathrm{p}=0.26\right.$, Figure A25). 


\section{Repeatability analyses}

$\log _{10}$ SVMP activity within individual snakes was almost perfectly statistically repeatable $(r=0.992, p<0.0001$, Figure $12 \mathrm{~A})$. As the repeatability statistic could be inflated due to wide variation between phenotypes, repeatability analysis was also conducted on each phenotype separately. Nonetheless, each phenotype was found to be highly repeatable (Low: $r=0.844, p<0.0001$; High: $r=0.967, p<0.0001$ ). $\log _{10}$ PLA2 activity levels were found to be highly statistically repeatable within individual snakes $(\mathrm{r}=0.7668, \mathrm{p}<0.0001$, Figure 12B $)$. Total protein concentration of venom is not reliably repeatable within individual snakes $(r=0.208, \mathrm{p}=0.0457)$, indicating that there is a great deal of variation within snakes that remains unaccounted for in this type of analysis (Figure 12C).

\section{DISCUSSION}

\section{CORT implant treatment elevated blood CORT levels in Treatment snakes}

CORT implant treatment in rattlesnakes elevated baseline plasma CORT levels in treatment snakes at the second sampling event, roughly 15 days after implantation. The magnitude of elevated baseline CORT in the treatment snakes at this sample is within

normal baseline levels based on reports from several species of free-ranging rattlesnakes, including the sister species C. oreganus (Holding et al. 2014a; Schuett et al. 2004; Lutterschmidt et al. 2009, Lind et al. 2010). As baseline CORT varies within inidividuals, within a population, and across seasons in these studies, it is difficult to attribute a specific value of baseline CORT to achieve a general classification of 
"chronically stressed". Thus, classification of "chronically stressed" animals only fit within a context of animals within the same population under the same environmental conditions. In this study, CORT-implanted snakes had elevated baseline CORT and thus can be considered to be exposed to levels of CORT indicative of chronically stressed animals, relative to the control group, for at least an average of 15 days. In endothermic avian reptiles, plasma CORT after implant can be elevated up to 15 days (Spee et al. 2011; Salvante \& Williams 2003), but also for as little as three to five days (Müller et al. 2009; Romero, Strochlic \& Wingfield 2005; DesRochers et al. 2009). In reptilian ectotherms, evidence for time release effects in CORT implants is mostly limited to before-after sampling. Some have been shown to elevate plasma CORT between three and four times baseline for ten days to three months (DeNardo \& Licht 1993; Cash \& Holberton 1999) but others report results without verifying elevation of plasma CORT levels during the study (Miles et al. 2007; DeNardo \& Sinervo 1994). One study attempted to investigate time-effects of CORT implant release, but failed to detect elevation in plasma CORT from implants (Juneau et al. 2015). This was likely due to a low recapture rate of CORT-implanted individuals due to opportunistic re-sampling rather than ineffectiveness of the implants, as the authors propose. Ours is the first study to experimentally elevate CORT via silastic implants for up to 15 days in a snake. Because CORT returned to baseline levels in the CORT-implanted group by the third sample (approx. 30 days), it is likely that the implant released most of the CORT between days 0 and 15 and had been depleted by day 30. Based on avian responses to CORT implants, there is a strong likelihood that plasma CORT in treated rattlesnakes between pre-implant and sample 2 was elevated to higher levels than detected at the second 
sampling (Müller et al. 2009; Romero, Strochlic \& Wingfield 2005; DesRochers et al. 2009). This means that the snakes in our study may have experienced higher CORT levels that the modest increase in CORT we observed at sample 2.

\section{Elevated CORT during synthesis did not affect venom activity or total protein}

\section{concentration}

Chronically elevated CORT due to implant treatment during venom re-synthesis did not affect ultimate venom activity of SVMP or PLA2 groups, and did not affect total protein concentration. Chapter 1 predicted increases in relative abundance of SVMP and decreases of PLA 2 with increased CORT, but the measured activities of these proteins did not correspond to CORT implant treatment. The change in activity and protein concentration from the pre-implant to second sample was also not correlated to change in CORT. We elevated baseline levels of treatment snakes by an average of $86 \%$, and while plasma CORT was likely elevated above this prior to the second blood sampling, we do not know how high these levels were, and whether they were sustained. Threshold levels of CORT required to induce fitness effects are not known (Jessop, Woodford \& Symonds 2013), and although increased CORT is implicated in many ontogenetic shifts, the magnitude and duration of CORT increase required to induce these changes likely differs across species and individuals (Wada 2008). It is possible that venom ontogeny in crotalids is an ultimate and irreversible change, similar to metamorphosis of amphibians (Wada 2008), such that no level of CORT would induce a change in adult snake venom because the change had already occurred. This hypothesis warrants investigation in neonate or juvenile snakes. Our study quantified the activity levels, but not abundances, of two protein families; it is possible that CORT implants affect relative abundance of 
venom proteins, but not activities (discussed in detail below). While we did not see an effect of implant treatment on venom protein concentration, this measurement cannot assess fine-scale changes in protein groups, as venom concentration is a measure of the amount of protein per unit fluid. The liquid components of venom appear to be regulated at a different time scale than protein production, and may be influenced by external factors as well. Protein concentration of venom is high early in the replenishment phase compared to several days later (Willemse et al. 1979; Currier et al. 2012), which is not likely due to destruction of just-synthesized proteins (Rokyta et al. 2015), but rather due to liquid components of venom increasing over time. CORT can cross-react with mineralocorticoid receptors, such as aldosterone receptors, which leads to water reabsorption into the blood stream (Thurnhorst, Beltz \& Johnson, 2007; Gaeggeler et al. 2005; Morris et al. 2000). If water is withheld from venom fluid when CORT is high, this could lead to an increased protein concentration of venom. However, if CORT also decreases relative protein abundance in venom (see Chapter 1, PLA2), no effect on total protein concentration would be detected, because both fluid and proteins would decrease. This type of response to elevated CORT could be adaptive, as it may ensure similar venom potency despite decreased overall venom yield during periods of starvation. Comparisons of venom yield, activity, total protein concentration, and relative protein abundances are necessary to fully investigate the potential regulatory effects of CORT on venom synthesis.

\section{Baseline CORT is associated with increased PLA $\mathrm{A}_{2}$ activity}

While implant treatment did not affect PLA2 activity, the baseline plasma CORT corresponding with each venom sample was a significantly positively related to PLA2 
activity. In Chapter 1, we demonstrated a possible negative relationship between CORT and PLA2 abundance. We predicted that protein activity would be correlated to protein abundance, that PLA2 would decrease in abundance with high CORT, and thus PLA2 activity would also decrease. While protein expression and abundance does not necessarily correlate to activity (Currier et al. 2010), CORT was recently demonstrated as a powerful in vitro substrate-binding inhibitor of PLAz type IIA isolated from Russell's viper venom (Shukla et al. 2015). Our results suggest an opposite, positive effect of baseline CORT on PLA2 activity. While we demonstrated elevated CORT in treatment snakes, it is possible that circulating CORT in a biological system does not reach the in vitro thresholds that elicited the magnitude of response on PLA2 activity demonstrated by Shukla et al. (2015). There are a variety of PLA2 proteins present in venoms (Sunagar et al. 2014), and it is possible that CORT-inhibition may be limited to the type IIA isoform of $\mathrm{PLA}_{2}$, but other isoforms remain to be tested. While it has been posited that posttranscriptional mechanisms do not contribute to differences in protein abundance (Rokyta et al. 2015), and that variation is largely attributed to a variety of coding sequences (Sunagar et al. 2014), differences in relative protein function due to post-translational modifications or inhibitors have not been thoroughly investigated in snake venoms.

In any given biological system, the proportion of active to inactive isoforms of a given protein may change to compensate for a change in protein abundance, and changes in protein abundance and in proportions of active proteins can be independent of one another (Glaneman et al. 2003; Currier et al. 2010). Activation of proteins is often achieved through phosphorylation by various protein kinases (Molecular Biology of the Cell, Alberts $6^{\text {th }}$ Ed). While CORT is known to influence gene expression through 
interactions with cofactors, it is also known to exhibit rapid, nongenomic effects, particularly in activation of protein kinase pathways in various biological systems (Li et al. 2001; Harms et al. 2007; Liu et al. 2010). CORT may exert dual actions within the same system, having a negative effect on transcription or translation of PLA2 proteins, but also a positive, nongenomic effect on PLA 2 activation pathways though kinase activation. In essence, an overall low abundance of $\mathrm{PLA}_{z}$ could be compensated for by activating a greater proportion of the existing PLA2 proteins. Further investigation is necessary to elucidate the mechanism behind the observed association of CORT and $\mathrm{PLA}_{2}$ activity.

From a functional perspective, increased activity of $\mathrm{PLA}_{2}$ may benefit a snake during periods of chronic stress. Due to the variety in number and structure, members of the snake venom family of PLA2 proteins collectively affect almost every body system when injected into a prey item (Kini 2003). Ubiquitous among PLA2 proteins are myotoxic effects, such as rapid necrosis of muscle tissue, while some also act as potent pre-synaptic neurotoxins (Lomonte et al. 2003; Kini 2003), and others have anticoagulant properties (Kini 2005). Increased relative activity may improve the toxicity of venom and the chance of securing a meal when baseline CORT is expected to be high, such as during periods of prolonged fasting or long-distance dispersal (Wingfield 2013; Busch \& Hayward 2009). In this context, upregulation of existing PLA2 in response to baseline CORT levels could be adaptive, especially if activation also compensates for low $\mathrm{PLA}_{2}$ abundance. To understand the broader implications of these relationships, quantification of protein abundance via HPLC techniques venom is necessary to 
determine if abundance and activation of venom proteins is changing concurrently in $C$. helleri.

\section{Effects of time and repeated sampling on protein activity and concentration}

Overall, SVMP and PLA2 activities were highly repeatable within individual snakes, in concordance with conclusions of Gregory-Dwyer et al (1986) and Chapter 1 of this thesis. This bodes well for researchers that rely on consistency of multiple samples from the same individuals for research or antivenom production (Chippaux, Williams, \& White 1991; Currier et al. 2012; Salazar et al. 2009). Although SVMP and PLA2 activity was highly repeatable within individual snakes, variation was evident among samples from the same individuals. Weak effects of time and repeated sampling were detected in all venom variables measured. SVMP activity was increased at third sampling relative to pre-implant sampling, but no difference was observed in PLA2 activity or total protein concentration across the three sampling periods. Repeated sampling within a span of days can affect the overall protein concentration of venom (Willemse et al. 1979), but does not appear to affect whole-venom activity (Currier 2012). At intervals of one month, overall protein composition is not affected by repeated sampling (Gregory-Dwyer 1986).

In this study, time between venom samples spanning 13 to 23 days was associated with a statistically significant increase in $\mathrm{PLA}_{2}$ activity and a non-significant increase in total protein concentration, but not with changes in SVMP activity. As both total protein concentration and PLA2 activity increased in these snakes, it is possible that complete translation of PLA2 proteins occurs over a longer timeline than SVMP, leading to a greater abundance of PLA2 in the venom of snakes with longer time to re-synthesize. These results, however, should be interpreted with caution as they were driven by three 
individuals. As significance disappeared when these snakes were excluded from analysis, this correlation should not be fully considered until protein abundance data are available. Other explanation for observed variation could be due to factors beyond researcher control in a field study. Gibbs et al. (2011) reported substantial changes in PLA2 composition in rattlesnake venom in response to a change in diet (Gibbs et al., 2011). Snakes in our study were not observed with food bulges between sampling periods, so all snakes likely fasted between samplings. Therefore, while inherent differences in diet may still explain variation among snakes, consumed prey likely did not affect repeatability of samples during the course of this experiment. However, we cannot rule out the possibility that snakes expended venom in defense or during unsuccessful predatory attempts, which may account for observed variation in activity or protein concentration.

\section{Association of snake size and SVMP activity adds complexity to current literature}

Ontogenetic shifts in expression of specific proteins within the SVMP and PLA2 classes are well documented (Alape-Girón et al., 2008; Gibbs et al. 2011; Guércio et al., 2006; Saviola et al., 2015), and are often attributed to the observed differences in SVMP activity phenotypes. In the current study, though we did not observe changes within snakes, we observed two distinct SVMP activity phenotypes. We detected a positive correlation between SVL and SVMP activity in the high SVMP phenotype group, which aligns with current evidence that Crotaline venoms are characterized by general increases in SVMP abundance as snakes grow larger (Mackessy 1988, but see Mackessy et al. 2003). However, there was no correlation between SVL and SVMP activity when both phenotypes of SVMP activity were included, showing that this relationship is not as clear-cut as predicted by previous literature. That is, equally large snakes were present in 
both observed high and low phenotype groups. Evidence also suggests decreases in PLA2 abundance with size for C. helleri (Mackessy 1988), but no correlation between PLA2 activity and SVL was detected in this study. Total protein concentration also appears to be unrelated to size differences. It is likely that a variety of biological cues influence observed differences in venom phenotypes.

\section{SVMP phenotype is related to difference in total SVMP protein content}

Our observation of two distinct SVMP activity phenotypes is consistent with evidence for wide variation in the specific SVMPs expressed and their corresponding levels of expression within individuals of the same species (Gibbs et al. 2009; Currier et al. 2010). It is likely that the observed gap in SVMP activity between the phenotypes is based on genetic variation in expression or expression levels (French et al. 2004; Currier et al. 2010; Mackessy 1996; Rokyta et al. 2015). Because total protein concentration was higher in high SVMP phenotype snakes, it seems likely that the difference in observed phenotypes could be attributed to differences in genotype, leading to expression of SVMP-encoding genes in high phenotype snakes that are not expressed in the low phenotype snakes. Current evidence, however, suggests that SVMP abundance or expression level does not correlate with activity differences (Currier et al. 2010). As we only investigated activities of two protein groups, and did not quantify abundances, the difference in protein concentration could be attributed to changes in abundance of other venom proteins. HPLC techniques identifying individual protein peaks within each family would be helpful in understanding whether presence of specific SVMP proteins in 'high phenotype' individuals drives the differences in SVMP activity within snakes in this population. 


\section{CONCLUSIONS}

To our knowledge, this is the first study to exogenously manipulate CORT via implants in snakes and the first to assess repeated venom samples from free-ranging individuals of any venomous snake. We show that SVMP, PLA2 activity, and protein concentration of $C$. helleri venom is largely unaffected when replenished while CORT is elevated due to the implants. However, we found a positive association of baseline CORT at sampling and PLA2 activity, regardless of implant treatment. The contrasting relationships between baseline CORT and PLA 2 relative abundance (Chapter 1) versus $\mathrm{PLA}_{2}$ activity raises the possibility that CORT may affect this proteins in this family at different levels of regulation, or that compensatory activation mechanisms exist to combat possible decreases in protein abundance due to CORT. Relative protein abundance measures from these data are necessary to understand this complex relationship. Total protein concentration appeared to be largely driven by phenotype differences observed in SVMP activity, suggesting that SVMP phenotype differences could be due to a difference in SVMP protein abundance. Ultimately, we found SVMP and $\mathrm{PLA}_{2}$ activity, but not total protein concentration, to be highly repeatable within individual snakes, although there were slight effects of time or repeated sampling in all venom variables tested. Even after exposure to a period of chronic stress via elevated baseline CORT, venom activity was highly repeatable. In the context of venom research for biomedical purposes, this result is reassuring that representative samples can be collected repeatably from the same individuals. However, much remains to be investigated about the potential nongenomic effects of CORT on venom activity. 


\section{FIGURES}

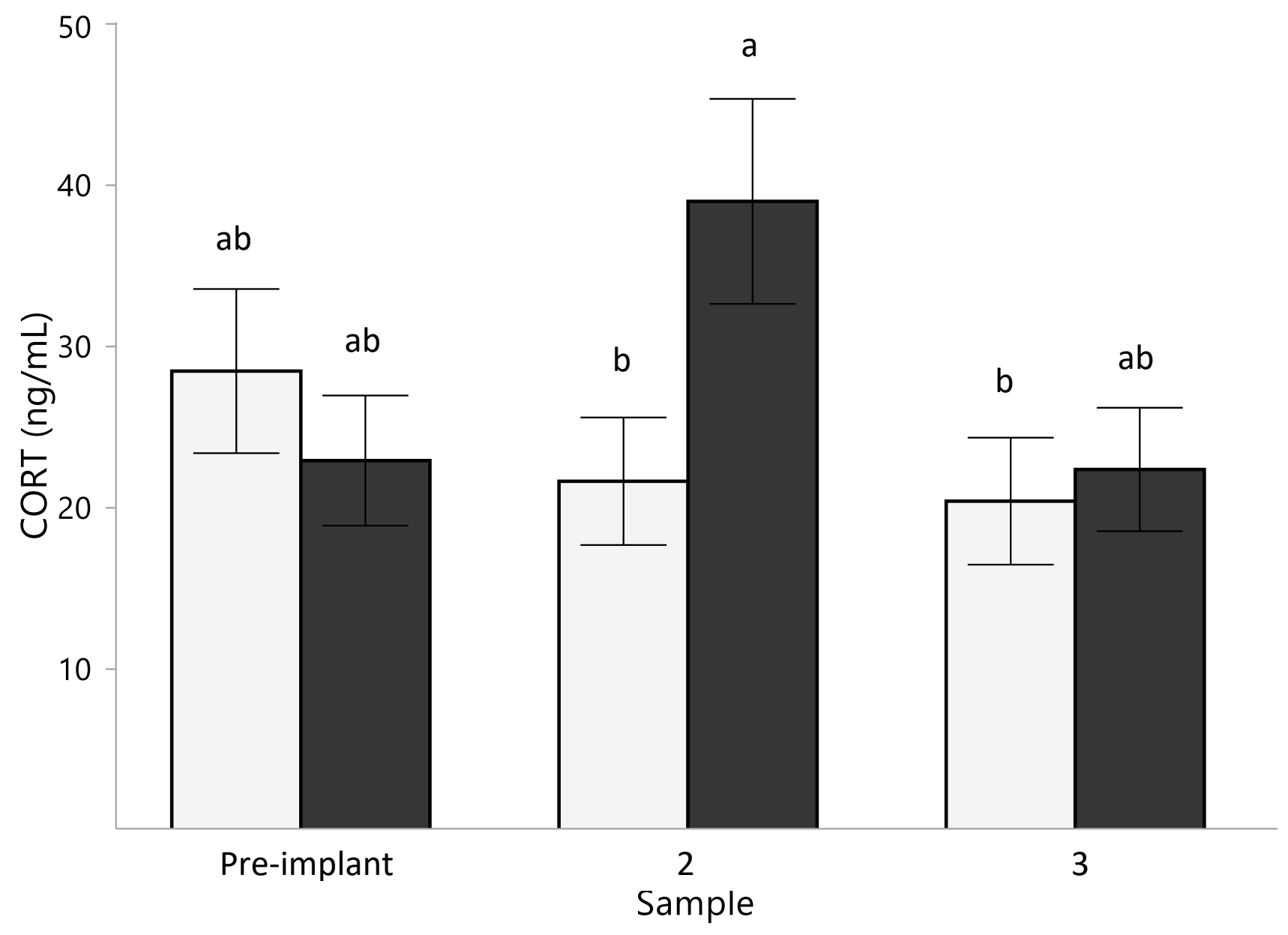

Figure 6. Baseline corticosterone (CORT) levels (ng/ml; +/- SEM) of control (light bars) and implant-treated (dark bars) Crotalus helleri across three sampling events. Baseline CORT was elevated in treatment snakes at the 15 day period after implant. Groups that do not share the same letter are significantly different. 

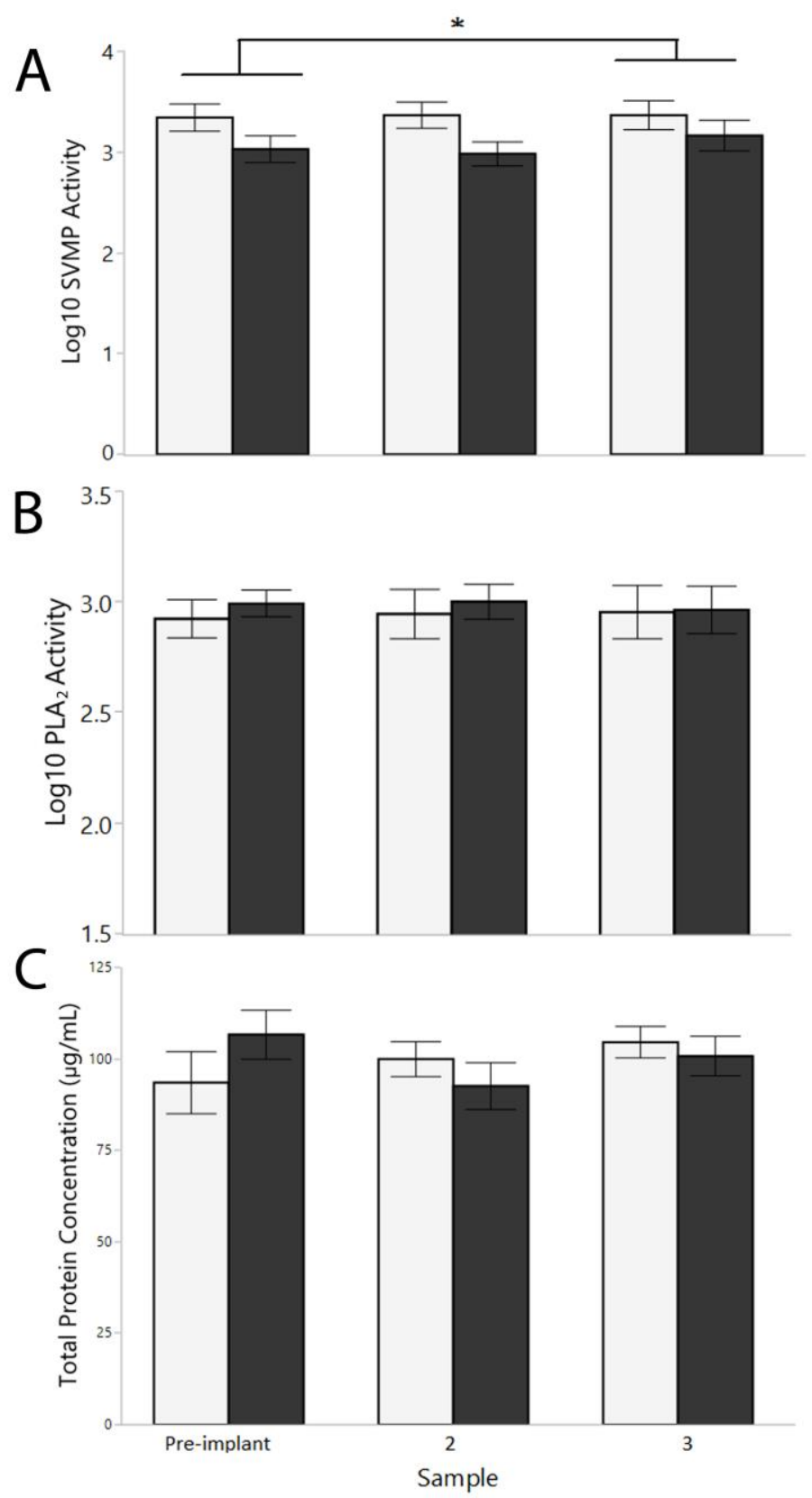

Figure 7. Mean protein activities (A,B) and total protein concentration (C) of control (light bars) and CORT-implanted (dark bars) Crotalus helleri at each sampling event. There were no differences in SVMP activity based on treatment. SVMP activity was higher in sample 3 than the pre-implant sample. There were no differences in $\mathrm{PLA}_{2}$ activity or total protein concentration based on treatment or repeated sampling. Asterisk indicates a significance difference. Error bars are \pm 1 SEM. 


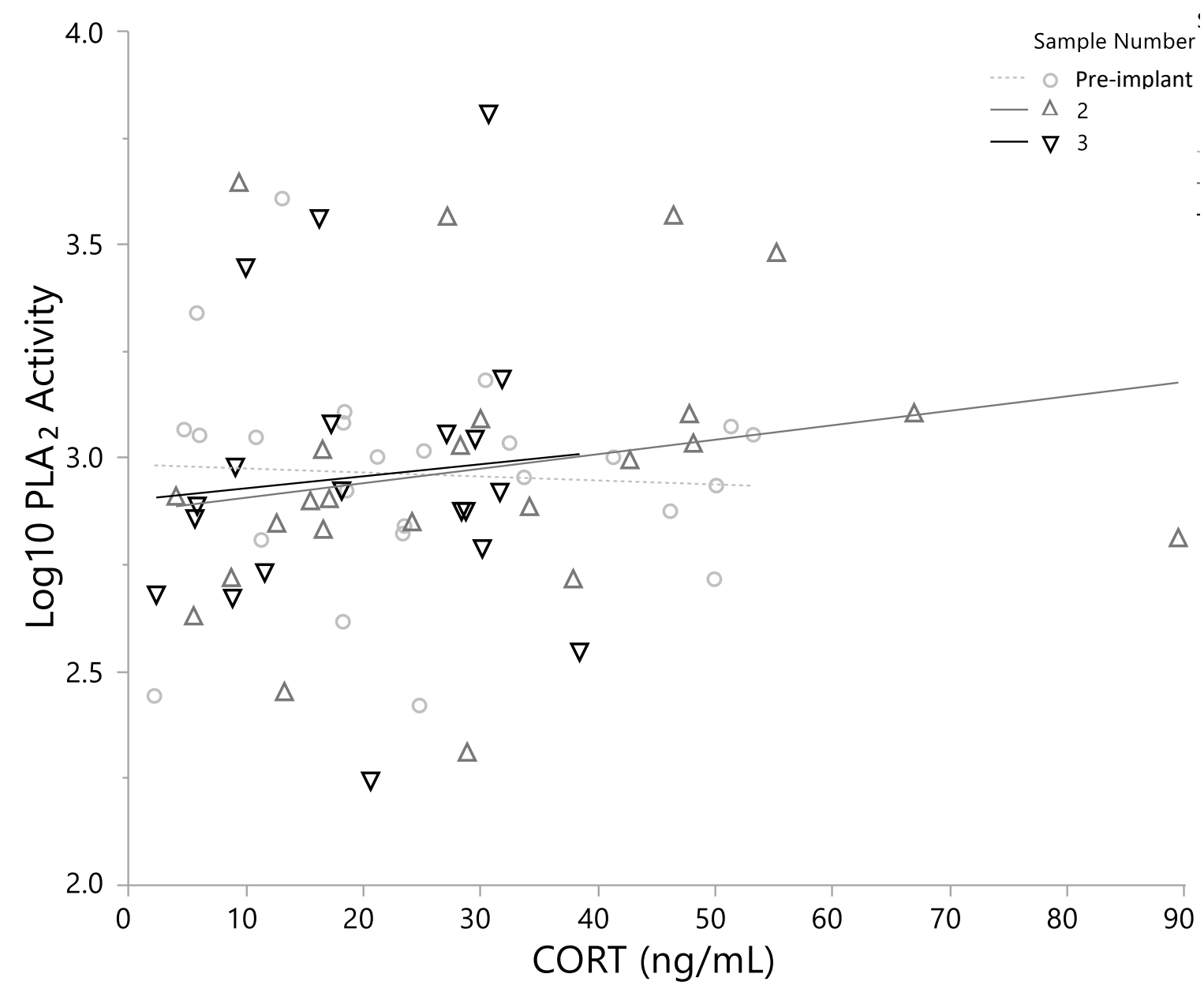

Figure 8. $\log _{10}$ transformed PLA 2 activity of Crotalus helleri plotted against baseline CORT values across all three sampling periods (in sequence from light to dark). While there was no main effect of sampling period, baseline sampled CORT was a significant covariate in the RM ANCOVA, with activity increasing with baseline sampled CORT, regardless of treatment group or sample period. Although all three samples from each snake are shown, each within-snake sample was not considered independent. The RM ANCOVA accounted for repetition within individuals, so there was no pseudoreplication in our dataset. 


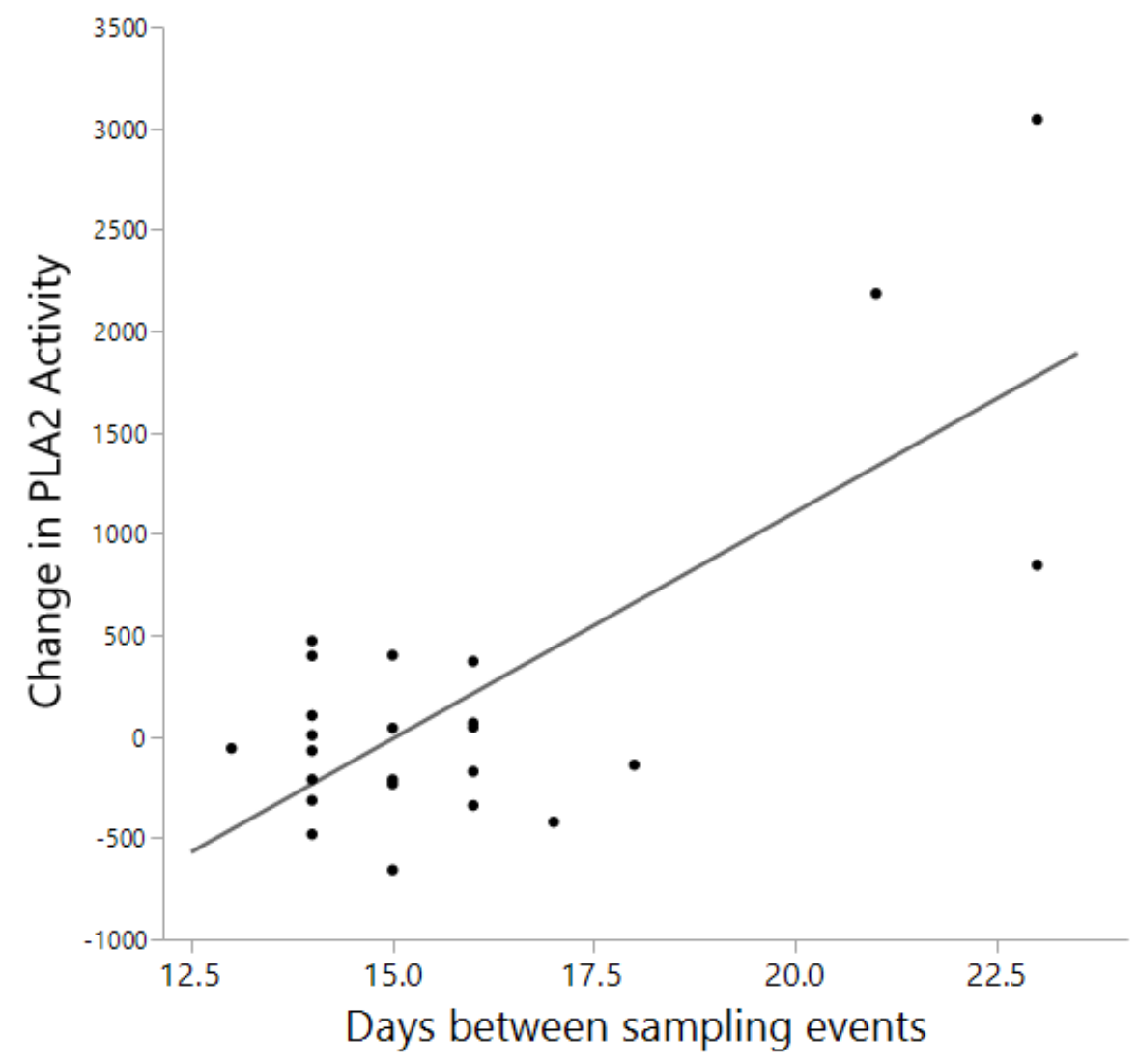

Figure 9. Change in $\mathrm{PLA}_{2}$ activity from pre-implant sample to sample 2 in Crotalus helleri. Days between sampling events significantly impacted the changes in activity, driven by 3 snakes with samples greater than 20 days apart. With these excluded, the relationship was no longer significant. 


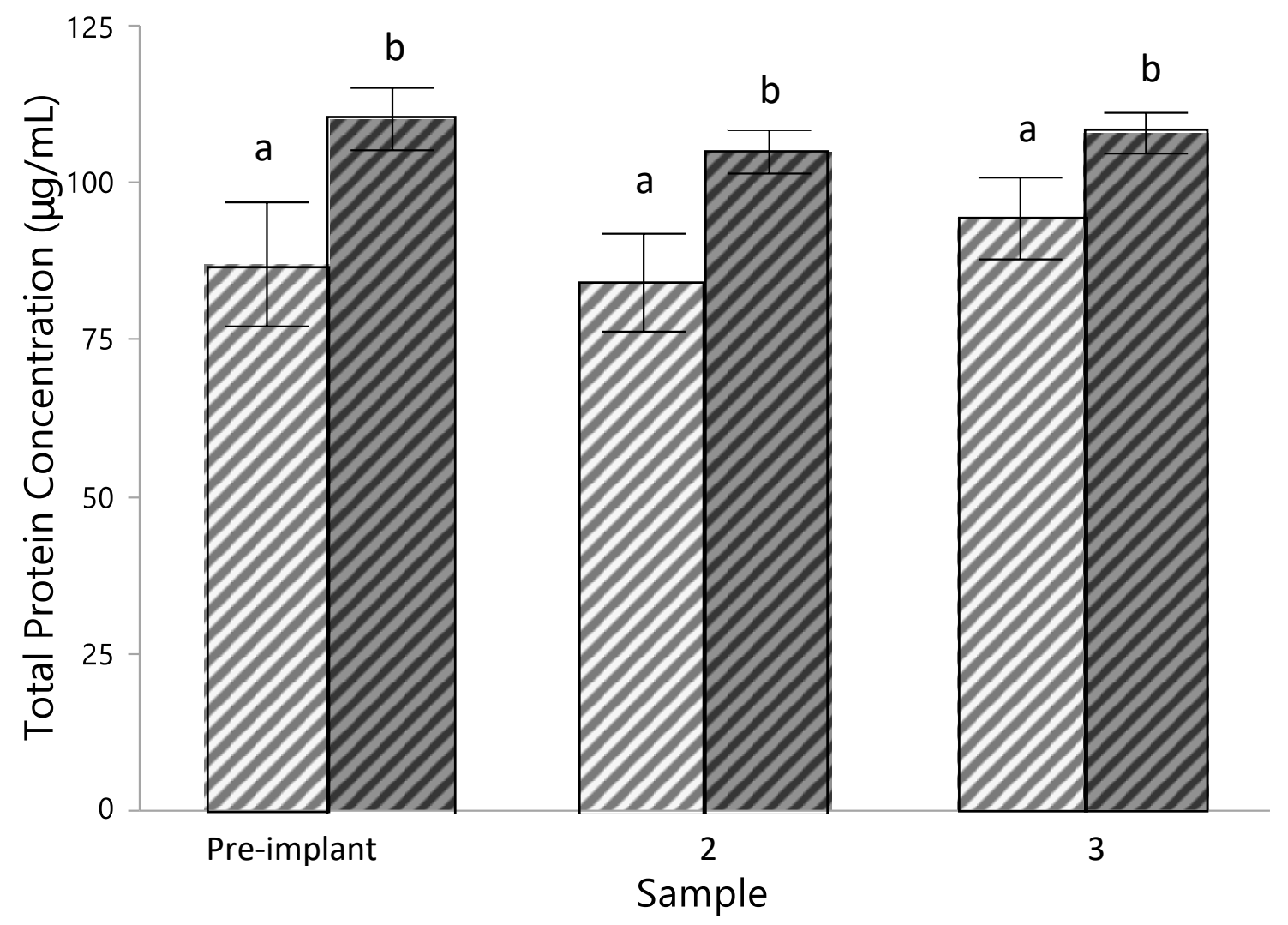

Figure 10. Mean total protein concentration of Low SVMP activity phenotype (light hatched bars) and High SVMP activity phenotype (dark hatched bars) Crotalus helleri. High SVMP activity phenotype snakes had higher total protein concentration of venom at all timepoints. Different letters indicate significant differences between groups. Error bars are \pm 1 SEM. 


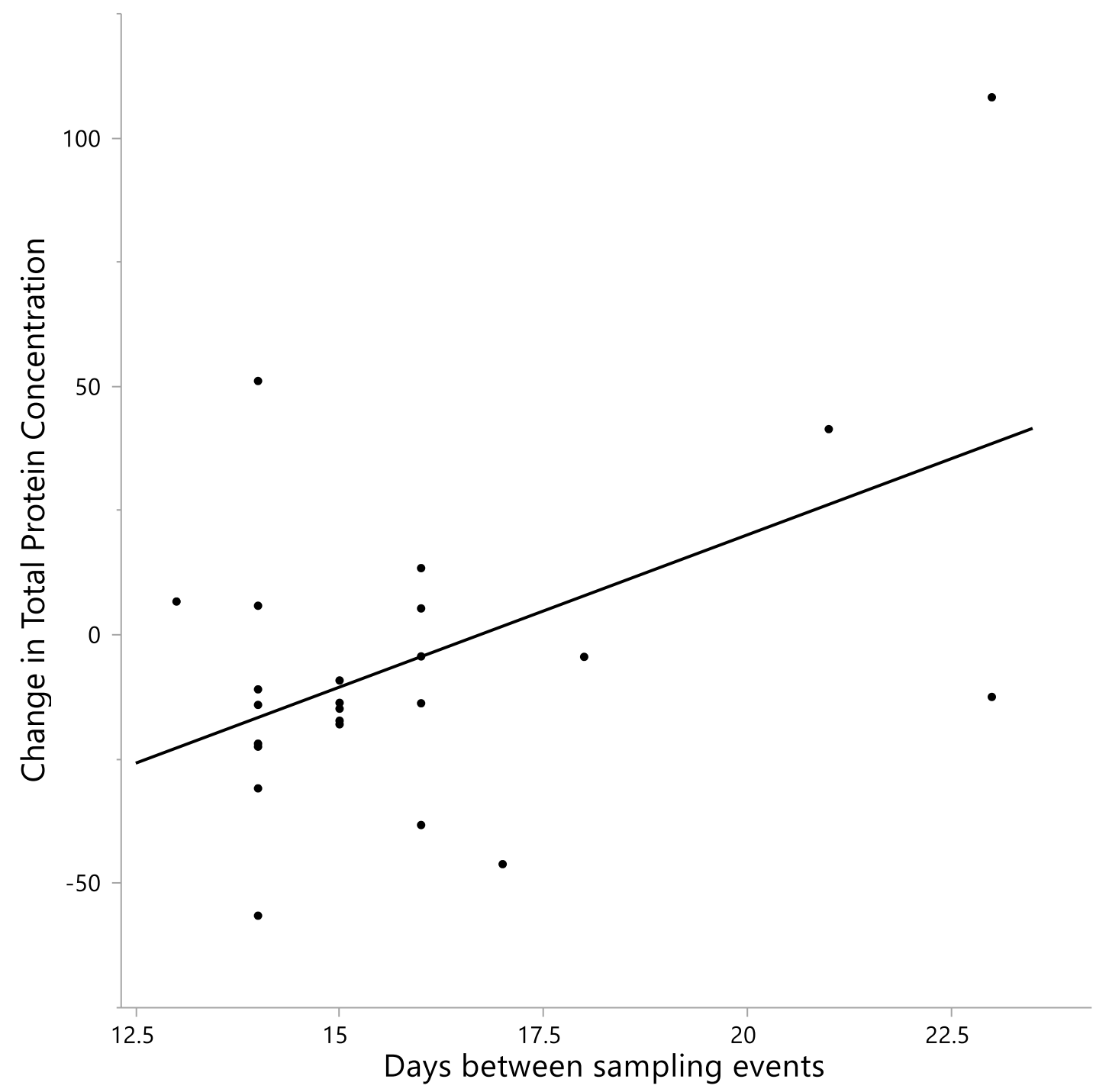

Figure 11. Change in total protein concentration from pre-implant sampling to sample 2 after implant in Crotalus helleri. There was a positive (but not significant) trend for effect of days between sampling events on total protein concentration driven by three snakes with samples greater than 20 days apart. 


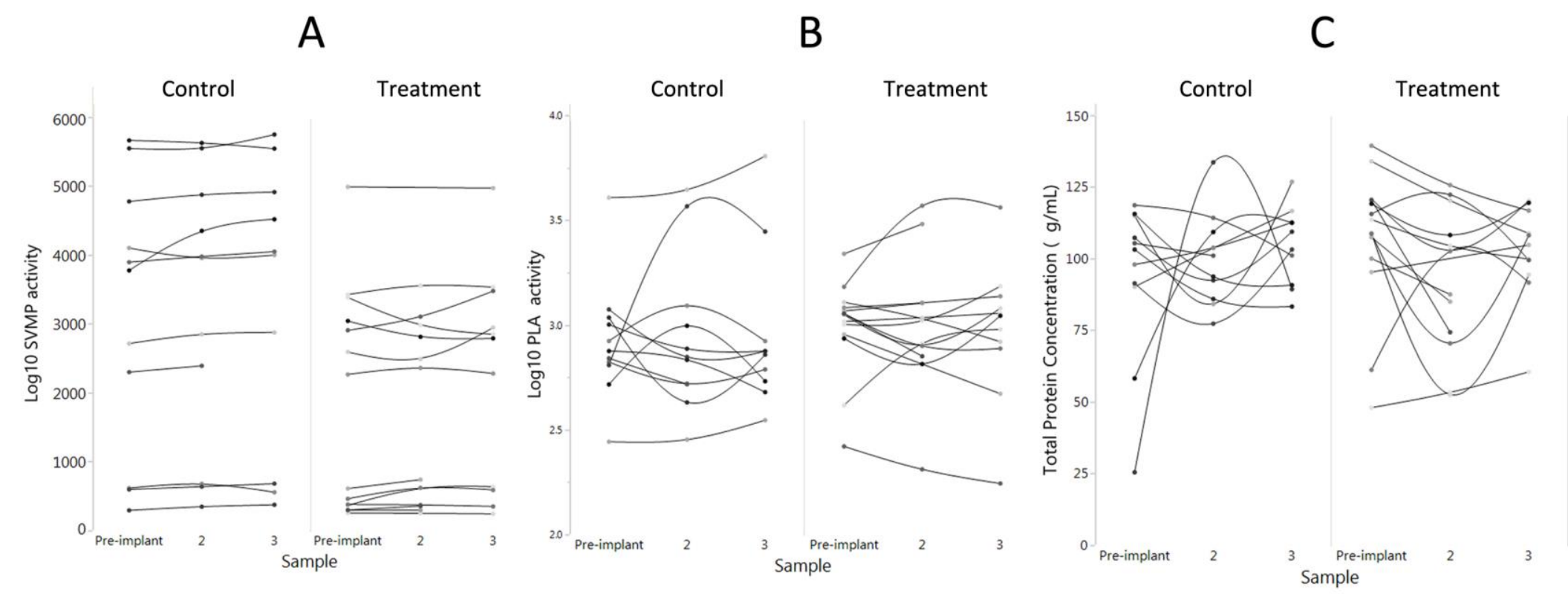

Figure 12. Individual response of Crotalus helleri venom protein activity (A, B) and total protein concentration (C) over time separated by treatment group. Each line depicts responses of an individual snake to repeated sampling across sampling periods. SVMP (A) and PLA 2 (B) activity was highly repeatable within individual $C$. helleri, regardless of treatment. Total protein concentration was not reliably repeatable (C). Note obvious gap in activities between $\log _{10}$ SVMP activity of 2.3 and 3.3, leading to our designation of "low phenotype" and "high phenotype" respectively. 


\section{CHAPTER III: EFFECTS OF CORTICOSTERONE IMPLANTS ON PHYSIOLOGICAL, BEHAVIORAL, SPATIAL, AND THERMOREGULATORY ECOLOGY OF CROTALUS HELLERI}

\section{INTRODUCTION}

Stress is a physiological state experienced by organisms in response to unpredictable or adverse conditions (Wingfield 2005). Glucocorticoid hormones including corticosterone (CORT) are elevated when an organism must cope with a stressor such as an encounter with a predator or low food availability (Moore, Lemaster \& Mason 2000; Berger et al. 2005; Hopkins \& DuRant 2011; Lutterschmidt et al. 2009; Baugh et al. 2014). Stress can be defined as acute-managed with short-term elevation of CORT — or chronic, resulting in long-term elevation of CORT levels above baseline (Dickens \& Romero 2013). During prolonged periods of chronic stress, such as during drought or extensive fasting, glucocorticoids are implicated in directing organism survival, triggering an emergency life history stage to mobilize energy stores and downregulate nonessential processes (Wingfield et al. 1998; Busch \& Hayward 2009). CORT levels can therefore be important indicators of the physiological state of freeranging animals and have been used to assess the health and status of vertebrate populations (Busch \& Hayward 2009; Jessop, Woodford \& Symonds 2013; but see Dickens \& Romero 2013), but the impacts of chronically elevated CORT are usually assumed, and have not been assessed in many taxa. 
Long-term elevated CORT has been reported to affect a suite of traits within a single organism, including reproduction (Wingfield \& Sapolsky 2003; Jones \& Bell 2004; Spée et al. 2011), immune function (Dhabhar 2000; Martin 2009; Seddon \& Klukowski 2012; McCormick and Langkilde 2014), behavior (Baird, Lovern \& Shine 2014), stamina (Cash \& Holberton 1999; Miles, Calsbeek \& Sinervo 2007), thermoregulation (Cree et al. 2003; Preest and Cree 2008), osmolality (Thunhorst, Beltz \& Johnson, 2007; Gaeggeler et al. 2005; Morris et al. 2000), and protein metabolism (Romero, Strochlic \& Wingfield 2005; Lorenz et al. 2009). Significant changes in any of these traits could affect survival or fitness of individuals (Escribano-Avila et al. 2013; Jessop, Woodford \& Symonds 2013) and their offspring (Cree et al. 2003; Salvante \& Williams 2003). However, many studies that attempt to induce chronic stress to observe its effects on animals actually use repeated acute stressors to elevate CORT, considering this to be the best approximation of chronic stress (reviewed in Dickens \& Romero 2013). However, sustained elevated CORT levels may represent a more physiologically relevant state of chronic stress. It is important from a physiological standpoint to understand how chronic stress is implicated in survival by understanding the many ways in which organisms respond to chronically elevated CORT, which may be different than the responses to many brief elevations in CORT (Dhabhar 2009; Seddon \& Klukowski, 2012). Studies on the effects of chronic stress on free-ranging ectotherms are largely lacking from the literature, largely due to difficulties in inducing chronically elevated CORT in wild animals.

We aimed to investigate effects of elevated CORT in the Southern Pacific rattlesnake (Crotalus helleri) due to its ability, like other rattlesnakes, to survive 
prolonged periods of drought and low food availability (McCue 2007). Ecologically, it is important to understand how arid-adapted ectotherms such as rattlesnakes cope with chronic stressors such as prolonged fasting, and whether CORT plays a major role in moderating trait expression for survival. Understanding responses of $C$. helleri to periods of chronic stress may also be important in predicting activity levels and relative risk of snakebite in a given year (Akani et al. 2013; Yañez-Arenas et al. 2016). To investigate the effects of CORT on rattlesnake physiological, spatial, thermoregulatory, and behavioral ecology, we exogenously manipulated CORT via implants in a repeatedmeasures study with radio-telemetered male $C$. helleri. Individual snakes were either treated with CORT implants or with blank implants, and effects on the snakes' behavior, sex hormones, movements, and body temperatures $\left(\mathrm{T}_{\mathrm{b}}\right)$ were assessed for 30 days. If CORT directs significant changes in aspects of rattlesnake ecology and follows general trends observed in other animals, we predicted that rattlesnakes implanted with CORT would have decreased testosterone due to previously reported suppressive effects of CORT on reproduction to prioritize immediate survival (Wingfield \& Sapolsky 2003, Jones \& Bell 2004); increased osmolality due to interaction of CORT with mineralocorticoid receptors (Thurnhorst, Beltz \& Johnson, 2007; Gaeggeler et al. 2005; Morris et al. 2000), increased defensive behavior to prioritize immediate survival, increased movements, home range sizes, and detectability due to mobilized energy stores and CORT stimulating dispersal from the area (Wada 2008; Hamann, Jessop \& Schäuble 2007), increased stress responses due to an increased baseline CORT (Dupoué et al. 2013; Sykes \& Klukowski 2009), and increased Tb relative to controls to facilitate mobilization of energy stores due to increased metabolic rates (Landys et al. 2006). 


\section{METHODS}

\section{Study animal and site}

Thirty adult male $C$. helleri were captured through visual encounter surveys at the University of California at Santa Barbara's Sedgwick Reserve in Santa Ynez, Santa Barbara County, California (34.6928 $\mathrm{N}, 120.0406^{\circ} \mathrm{W}$, elevation: $\left.290 \mathrm{~m}\right)$ from mid-April to early May 2015. This field site was chosen because fresh water sources are available for wildlife year-round, thus snakes were unlikely to be chronically stressed due to reduced foraging options prior to treatment (Jessop et al. 2013; Sperry \& Weatherhead 2008) compared to other sites in California, which suffered extreme drought for several years prior to the study. Snakes received intra-muscular passive integrated transponder tags (MUSICC Chip, AVID Identification Systems, Inc., Norco, CA, USA), and the three basal rattle segments were filled with non-toxic acrylic paint to a predetermined color

code for future identification. The snakes at the site were found mainly in cattle-disturbed valley oak savannah habitat, with areas of chaparral and coastal sagebrush. Rattlesnakes were collected under California Department of Fish and Wildlife Scientific Collecting Permit \# SC-13134, and experimental procedures were approved by the California Polytechnic State University Institutional Animal Care and Use Committee (Protocol \#1416) and the University of California at Santa Barbara Institutional Animal Care and Use Committee (Protocol \#Taylor 1415, Animal Activity \#027).

Snakes were transported to California Polytechnic State University for radiotransmitter and thermal data logger implantation. Snakes were housed individually in 30" x 12" x 12" Visionarium cages (Vision Products) with heat pads, hide boxes, and water ad libitum. Snakes were anesthestized via isofluorane inhalation (Vet One, MWI, 
USA) and received intra-coelomic implants of a $3.25 \mathrm{~g}$ temperature data logger (Thermochron iButton model DS1922L\#F50, Maxim Integrated, San Jose, California) and a radiotransmitter weighing 5.3, 11, or $13.5 \mathrm{~g}$ (Holohil Systems Ltd., Carp, Ontario, Canada) depending on snake body size. Combined weights of implants totaled less than $5 \%$ of snake total body mass. Snakes were released at the site of capture 1-2 days after surgery.

\section{Experimental administration of exogenous corticosterone}

Snakes were allowed to recover in the field for at least two weeks post-surgery prior to experimental manipulation in late May 2015. Snakes were divided into two mass classes at $800 \mathrm{~g}$, which was the average and median weight for all snakes captured. Snakes greater than $800 \mathrm{~g}$ were given two $15 \mathrm{~mm}$ (large) implants, and snakes less than $800 \mathrm{~g}$ received two $7.5 \mathrm{~mm}$ (small) implants. Snakes within each weight class were randomly assigned to either a treatment group or control group. Treatment snakes received implants filled with CORT, while control snakes received blank implants. Seven large snakes and eight small snakes were assigned to the CORT treatment group. Eight large and seven small snakes were assigned to the control group.

Corticosterone implants were made by plugging one end of a $15 \mathrm{~mm}$ or $7.5 \mathrm{~mm}$ silastic diffusion tubing (Dow Corning, Clarkesville, TN, USA: $1.47 \mathrm{~mm}$ inner, $1.96 \mathrm{~mm}$ outer diameter) with $2 \mathrm{~mm}$ silicone caulking (Momentive Performance Materials Inc., Huntersville, NC), then allowed to dry for 24 hours. The tubing was then filled with crystalline CORT (Sigma C2505-500 mg Lot\# SLBJ5337V), and plugged with 2mm of silicone on each end and allowed to dry for 24 hours. Control implants were left empty, then plugged with silicone. For each size class, triple the amount of implants required 
were created. Implants were weighed, with the upper and lower third of implants discarded to improve dosing accuracy. $15 \mathrm{~mm}$ treatment implants averaged $6.1 \mathrm{mg}$ CORT, while $7.5 \mathrm{~mm}$ treatment implants averaged $3.6 \mathrm{mg}$ CORT.

Implants were soaked in $0.9 \%$ saline solution for 12 hours prior to implantation to facilitate CORT release. Each snake received two implants. The first implant was designed as fast-release by poking one small hole in the tubing with an insulin syringe. The second implant was left intact as a slow-release dose. In the field, snakes were captured and implants were injected intra-coelomically on each side of the lower third of the body via a sterilized $12 \mathrm{~g}$ PIT tag injector. Maximum total dosage of large CORTtreated snakes averaged $11.9+/-1.9 \mathrm{mg} / \mathrm{kg}$, while small CORT-treated snakes ranged 14.7 $+/-4.2 \mathrm{mg} / \mathrm{kg}$.

\section{Sampling period - Capture data}

Each snake was sampled initially on the day of implantation immediately before implants were administered (pre-implant sample), then approximately 15 days (sample 2) and 30 days post-implant (sample 3). Observers were blind to treatment assignment of snakes. Prior to each capture event, a behavior assay was performed. Snakes were then captured using tongs (Whitco Manufacturing Inc., Weatherford TX, USA) and tubed for safe restraint (Plastic Restraining Tubes, Midwest Tongs, Greenwood, MO, USA). Immediately after tubing, cloacal temperature was measured with a thermometer (SmartHomes Digital Cooking Thermometer, Divinity In Motion, location unknown), snakes were bled, snout-vent-length (SVL) and mass were measured, and venom was collected for a separate study (See Chapter 2). Snakes were immediately released at the 
site of capture, except for sample 3, where snakes were held captive for one hour to perform an acute stress series test.

Only snakes above ground or visible in a burrow were captured to avoid disturbing habitat and inducing unequal stress responses across individuals. A few snakes were unavailable for capture during the sampling period, as they were deep underground in burrow complexes. These snakes were sampled at the next possible opportunity, and then sampled within a ten to fifteen day period thereafter to maintain consistent venom regeneration time across individuals for a separate study (See Chapter 2). Snakes closer to the surface were excavated from burrows if the process was estimated to take less than ten minutes from start of digging to blood sample collection. Samples from snakes that were excavated are not included in behavior assay analysis. Two snakes were unavailable for sampling, and one snake died of an injury likely caused by predation. This resulted in seven small and seven large snakes in the treatment group, and eight small and five large snakes in the control group. One large control snake only had an initial sample, but its data were used in some parts of the analysis.

Snakes were recaptured in October and November 2015 for radiotransmitter and data logger removal and released at their sites of capture after recovery.

\section{Blood}

Time from beginning of handling (or disturbance of habitat) to blood drawn (time-to-bleed) was recorded. A $0.5 \mathrm{ml}$ sample of blood was collected via puncture of the caudal vessels with a 25 gauge $3 / 4$ " syringe at each capture. Within 48 hours, plasma was separated from whole blood via centrifugation at 10,000 rpm for three minutes. Plasma 
osmolality was determined with a freezing point depression osmometer (model 3320, Advanced Instruments, Inc., Norwood, Massachusetts). Plasma was stored at $-20^{\circ} \mathrm{C}$ until radioimmunoassay (see below). Time of day was recorded to account for variation due to diel CORT cycling (Lutterschmidt \& Mason 2010).

\section{Radioimmunoassay of blood plasma}

CORT and testosterone (T) levels were determined via radioimmunoassay of plasma as described in Lind et al. (2010). Briefly, samples were extracted in dichloromethane, then dried in a $40^{\circ} \mathrm{C}$ water bath under nitrogen gas. Samples were incubated overnight in $100 \mu \mathrm{l}$ of antiserum (CORT: Esoterix Endocrinology, Calabasas Hills, CA; T: T-3003, Wien Laboratories, Flanders, NJ 07876) and 100 $\mu$ l of tritiated steroid. Unbound steroid was separated from bound steroid using dextran-coated charcoal. A liquid scintillation counter was used to count bound steroid in samples, and final concentrations were corrected for extraction efficiency. Mean recovery for CORT was $64 \%$, $\mathrm{T}$ was $60 \%$. Serial dilutions for the standard curves were performed in triplicate $($ CORT curve range $=2000-4 \mathrm{pg}, \mathrm{T}: 500-1 \mathrm{pg})$. The limit of detection for CORT was $2.5 \mathrm{ng} / \mathrm{ml}$, T was $0.75 \mathrm{ng} / \mathrm{ml}$, and the intra-assay coefficient of variation (CV) was $7.3 \%$ for CORT and $11.14 \%$ for $\mathrm{T}$.

Repeated measures ANCOVA were performed with baseline CORT, Osmolality, and Testosterone as response variables using sample number (pre-implant, sample 1, sample 2) to account for changes in time, and Snake ID included as a random factor. Time-to-bleed was log-transformed, and SVL was squared to meet assumptions of normality and homogeniety of variances Time of day, time-to-bleed, and body temperature were included as covariates in the repeated measures ANCOVA. One 
treatment snake was excluded from CORT analysis, as only a pre-implant sample was collected, and no other samples were available from this individual for repeated-measures analysis. Dixon's Q test identified one outlier in the dataset with exceedingly high CORT levels at sample 3 (Dean and Dixon 1951; SAS Institute, Cary, NC 2014). This sample was excluded from the dataset for analysis. Sampling data were organized into three events: pre-implant, sample 1 (about 15 days post-implant), sample 2 (about 30 days post-implant). Separate repeated measures ANCOVA were used to determine the effects of CORT implant.

\section{Acute stress series}

To evaluate potential effects of implant treatment on stress reactivity, a standardized acute stress series (Holding et al. 2014a) was performed on each snake after collecting blood and $\mathrm{Tb}$ at sample 3 by placing each snake in a pillowcase in opaque buckets while the search for additional snakes continued. After one hour in the bucket, snakes had cloacal temperatures measured and were bled to obtain stressed samples.

A repeated measures ANCOVA was performed with sample number (initial vs. stressed) accounting for time, and Snake ID included as a random factor. We also calculated the magnitude of CORT change due to acute confinement stress (by subtracting baseline CORT from stressed CORT) and used this variable as a response in a separate one-way ANOVA to examine fine-scale differences in acute CORT-secretion.

\section{Defensive behavior}

Specific snake behaviors were recorded as they occurred during standardized capture events. These behaviors included tongue flicking, retreating (attempting to flee 
down a burrow or move in a direction opposite of the observer), rattling, striking (lunging open-mouthed at the observer, tongs, or tube), and head hiding (placing head underneath coils of body). These behaviors were noted upon initial sighting of the snake, during 5 seconds where the snake was squeezed with tongs (tong hold), during 5 seconds of lifting the snake several inches off of the ground, and during tubing attempts (variable lengths of time). Behaviors were ranked and assigned a point value according to their degree of defensiveness, on a scale from alertness to self-preservation (Tongue Flick=1, Retreat=2, Rattle=3, Strike=4, Head Hide=5). Scores were added for each event, with higher scores representing more behaviors exhibited (ex: If snake displayed both rattle and tongue flick, the score equaled 4), and thus a higher level of defensiveness. Defensive scores from each event (sighting, tong hold, lift, tubing) were correlated to the event preceding it, so the average of the score across the entire assay was used for analysis. If a snake was under cover, behavior was not scored. If a snake was dug out of a burrow or out of vegetation, its behavior was not included in the analysis.

\section{Defensive behavior during tracking period}

Defensive behavior scores at sighting were recorded during all tracking events, but the remainder of the assay (eg. tong touch, lift, tubing) was only performed on days that snakes were sampled for blood to avoid disturbing the snakes unnecessarily between sampling periods. Due to this, defensive behaviors recorded on sighting during tracking days were pooled and averaged by period (Period 1: 0-15 days post-implant, Period 2:1630 days post-implant) for analysis via repeated measures ANCOVA. Models were tried with each of the $\mathrm{T}_{\mathrm{b}}$ variables (maximum, minimum, average, range) from each period, including only one at a time to prevent multicollinearity. Only one $\mathrm{T}_{\mathrm{b}}$ variable (minimum 
$\mathrm{T}_{\mathrm{b}}$ ) reached significance as a covariate, and none of the other factors' or covariates' significance was affected by substituting the different $T_{b}$ variables, so minimum $T_{b}$ is reported as a covariate in the final model.

Defensive behavior at sampling

A separate RM ANCOVA analysis was performed for snakes from the three sampling times, as at these times, snakes were subjected to the full behavior assay. For the behavior-at-sample analysis, each step in the assay was correlated to the value of the next (i.e. a snake that rattled during lift was likely to continue rattling during tubing). Therefore, the average score of all four steps was used to calculate the average defensive behavior score at each sample. In both the tracking period and sampling analyses, defensive behavior score was square-root transformed.

\section{Movement, home range, detection probability}

Prior to implantation, snakes were tracked weekly or bi-weekly. Snakes were tracked daily during the time between initial implant and sample 3 (about 30 days) but were not tracked on days immediately after a sampling day. Snakes were tracked biweekly to weekly thereafter to assess any long term effects of implantation on home range size. Snakes were tracked an average of $42+/-3.8$ times (S.D.) throughout the duration of the study over an average of $150.8+/-20.9$ days (S.D.). The variation in tracking events was due to radio signals being lost on several snakes throughout the study period, perhaps due to blocked signals when snakes sheltered in metal piping and debris present at the field site. Variation in number of total tracking days is due to variation in

final collection date of snakes for radiotransmitter removal, which began October 3 and 
lasted until Nov 22 when the last snakes were captured. In all analyses, number of GPS points was included as a covariate to account for any bias in tracking effort. One snake's signal was permanently lost in mid-July, and another snake was found dead in August, which reduced sample sizes further to seven small and seven large snakes in the treatment group, and seven small and four large snakes in the control group for the long-term analyses. Both of these snakes are included in sampling period data analyses, but excluded from long term analyses.

During each tracking event, observers visually searched for the snakes, but did not move vegetation or overturn cover to avoid physically disturbing unseen snakes. Detectability of snakes was recorded based on whether the snake was visually located after tracking effort. Radiotelemetry allowed each snake's general location to be found, but we were interested in whether snakes were above ground or exposed, thus being visually detectable to an observer. Once a snake was visually located, the snake was marked as "detected", and body positions and defensive behaviors (see above) were recorded for each detected snake. Snakes were not handled upon detection unless required for sampling. If the snake was not seen (i.e., underground, or under dense cover), it was marked as "not detected". For each snake, detectability was calculated as the proportion of total detections divided by the number of tracking events for each period.

Snake locations were recorded using a Garmin eTrex Legend H model and GPSmap 62stc (Garmin Ltd) and converted for use in geographic information system (GIS) software. Spatial analysis was conducted in ArcMap 9.3 (ERSI, Redlands, CA, USA). Snake home ranges were calculated as $100 \%$ or $95 \%$ minimum convex polygons 
(MCP) using the Home Range Tools extension for ArcGIS (Rodgers et al. 2007). These polygons are commonly used with rattlesnakes, as they are relatively sedentary and do not move far beyond each sighting event (Holding et al. 2014a). MCPs for two periods post-implant (0-15 days and 16-30 days) were calculated to assess total area covered by snakes during this period, but only $100 \%$ MCP was used in the RM ANCOVA analysis, as $100 \%$ and $95 \%$ MCPs did not differ. Pre-implant home ranges were not assessed, due to a shortage of GPS points for MCP calculation. We also calculated home range sizes over the entire field season, reporting both $100 \%$ and $95 \%$ MCPs as responses in separate one-way ANCOVAs. In both short and long-term analyses, MCPs were log-transformed to meet assumptions of normality and homogeneity of variances.

Total distance moved was calculated with the measure tool with 'snap-to' feature enabled between each location. Only distances of greater than 10 meters between points were used to calculate total distance moved to account for the error in the GPS. A repeated measures ANCOVA was used to evaluate total distance moved and total area covered between Periods 1 and 2 post-implant. To evaluate distances moved before treatment, a separate one-way ANCOVA was performed on the 15 days prior to treatment. Pre-implant analysis was conducted separately due to differences in tracking effort between pre- and post-implant periods. In both cases, total distance moved was square-root transformed to meet assumptions of normality and homogeneity of variances.

\section{Temperature}

Hourly $\mathrm{T}_{\mathrm{b}}$ were recorded with surgically implanted iButton thermal data loggers in each snake. Daily maximum, minimum, range (minimum subtracted from maximum), and average $\mathrm{T}_{\mathrm{b}}$ were averaged and analyzed with RM ANCOVA by period (Pre-implant: 
-15 to 0 days, Post-implant 1: 0-15 days and Post-implant 2: 16-30 days). Range in $\mathrm{T}_{\mathrm{b}}$ was log-transformed to meet assumptions of homogeneity of variances. To better understand thermoregulatory ability, we also modeled daily fluctuation in $\mathrm{T}_{\mathrm{b}}$ by hour for each pre and post-implant period using sine and cosine of hour to model the circular movement of time (i.e., temperature at hour 0 is related to hour 23). If significant effects were detected, the model was run with "hour" replacing "sine time" and "cos time" to understand at which hours differences were occurring. $T_{b}$ fluctuation analysis was also conducted at weekly intervals to account for small-scale differences within post-implant periods. In both fluctuation analyses, $\mathrm{T}_{\mathrm{b}}$ was squared to meet assumptions for homogeneity of variances. Air temperature $\left(T_{a}\right)$ data were collected from Sedgwick

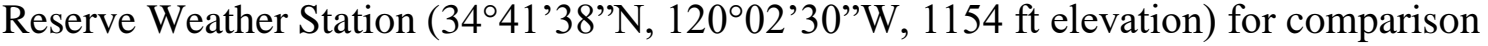
to $\mathrm{T}_{\mathrm{b}}$ data.

\section{Model selection and organization of data}

All statistical tests were performed in JMP Pro 12 (SAS Institute, 2015). The following transformations were applied to covariates to meet assumptions of homogeneity of variances: time-to-bleed: log; SVL: squared. Relevant terms were included in the model, and non-significant terms were systematically eliminated only if this improved the model's significance. If Treatment was the least significant term in the model, all of the relevant covariates were kept in the model. Only significant results are reported in the text. Non-significant factors and covariates included in final models are reported in Table 2. 


\section{Sampling data}

Sampling data were organized into three events: pre-implant, sample 1, sample 2. Post-implant sample 1 was usually 15 days and post-implant sample 2 was usually 30 days after initial implant, but due to variation in time between samples (i.e. for postimplant sample 1, some snakes were underground on day 15 , and were not available for sampling until day 17), we refer to each by sample by numbers 1 and 2 .

\section{All models}

Due to the repeated measures design where the first sample and period 0 from all snakes is pre-implant, 'Treatment by sample or treatment by period interaction' refers to the effects associated with CORT implants over time. 'Treatment' refers to the main effect of the CORT implant treatment, "sample" refers to the main effect of time based on samples collected at pre-implant, sample 2 and sample 3. Data collected continuously (i.e., not only at the three sampling periods, such as movement and $\mathrm{T}_{\mathrm{b}}$ ) are grouped and analyzed by 15-day periods. In these data, "Period" will refer to a main effect of time collected between sampling events.

Size class was included in all models as a main effect in the analyses, to account for the method of experimental randomization, but these results are not reported. SVL was included as a covariate to account for continuous variation due size differences. Body condition was calculated by dividing $\log ($ mass $)$ by $\log (\mathrm{SVL})$. If an interaction or categorical variable was significant, Tukey's Post-hoc tests were used to determine groups that were significantly different from one another. Student's t-test was used when there were only two groups to compare. 


\section{RESULTS}

\section{Effect of CORT implants on blood hormone levels}

CORT levels were elevated by treatment at sample 2

Compared to pre-implant treatment levels, CORT was elevated in treatment group snakes at Sample $2\left(\mathrm{~F}_{2,41.35}=3.33, \mathrm{p}=0.046\right)$ by an average of $60 \%$ higher than control snakes (Figure 6). By Sample 3, CORT had returned to pre-implant levels in treatment snakes. The main effect of treatment was marginally non-significant $\left(\mathrm{F}_{1,22.43}=3.58\right.$, $\mathrm{p}=0.07$ ). Figure 13 shows the overall dose-response curve of CORT levels in treatment versus control snakes, while figure 14 shows individual snake response to implant treatment over time.

Implants did not affect $T$ levels

There was no sample by treatment interaction on $\mathrm{T}$ levels, nor was there a main effect of treatment assignment or sample number (Figure A27). Size class was a significant main effect $\left(\mathrm{F}_{1,21.99}=8.64, \mathrm{p}=0.008\right)$, with larger snakes showing higher testosterone levels. However, SVL was not a significant covariate, so this effect is only seen when snakes are binned into coarse measurements of size. Time of day was a marginally non-significant covariate $\left(\mathrm{F}_{1,46}=3.05, \mathrm{p}=0.09\right)$, with a trend for decreasing $\mathrm{T}$ late in the day (Figure A28).

\section{Osmolality}

Sample was a significant main effect $\left(\mathrm{F}_{2,45.09}=8.16, \mathrm{p}=0.009\right)$, with pre-implant osmolality higher than the two subsequent samples for both treatment groups (Figure 16). 
There was no treatment by sample interaction, nor a main effect of treatment on plasma osmolality. Time-to-bleed was a significant covariate $\left(\mathrm{F}_{1,56.65}=8.04, \mathrm{p}=0.006\right)$, with osmolality increasing with increasing time-to-bleed (Figure 17).

\section{Trend for baseline CORT affecting magnitude of stress reactivity}

All snakes elicited a stress response to the bucket procedure $\left(\mathrm{F}_{1,19.69}=50.30\right.$, $\mathrm{p}<0.0001$ ), but there was no main effect of treatment and no effect of treatment by sample interaction (Figure 18). We also analyzed the magnitude of the stress response using a one-way ANCOVA. Initially, time of day, time in bucket, and initial $\mathrm{T}_{\mathrm{b}}$ were included in the model as covariates, but none were significant, so were removed to improve model power. There was no effect of treatment. Baseline CORT (before snakes were exposed to the acute confinement stress $)$ was marginally non-significant $\left(\mathrm{F}_{1,15}=3.36\right.$, $\mathrm{p}=0.09$ ), with a trend for snakes with lower baseline CORT to show a greater magnitude

of stress response (Figure 19). Body temperature change between samples was marginally non-significant $\left(\mathrm{F}_{1,15}=2.30, \mathrm{p}=0.09\right)$, with snakes that increased in body temperature tending to show a greater magnitude of stress response (Figure 20).

\section{Defensive behavior}

\section{During tracking period}

There was no effect of treatment, post-implant period, or a treatment by sampling period interaction on defensive behavior observed during tracking (Figure A31). Average range in $\mathrm{T}_{\mathrm{b}}$ was a significant covariate $\left(\mathrm{F}_{1,18.93}=5.20, \mathrm{p}=0.03\right)$, with snakes exhibiting smaller fluctuations in daily $\mathrm{T}_{\mathrm{b}}$ exhibiting greater defensive behavior (Figure 21). 


\section{Defensive behavior assay during sampling}

There was no main effect of treatment, sample number, or a treatment by sample number interaction (Figure A32). Testosterone was a significant covariate $\left(\mathrm{F}_{1,26.86}=5.87\right.$, $\mathrm{p}=0.02$ ), snakes with higher testosterone had higher defensive behavior scores (Figure 23).

\section{Total distance moved}

During the pre-implant period, snake movement was not significantly different between treatment groups. Snakes moved more during period 1 than during period 2 after implantation $\left(\mathrm{F}_{1,21.19}=7.96, \mathrm{p}=0.01\right.$; Figure $\left.24 \mathrm{~A}\right)$. Body condition was a significant

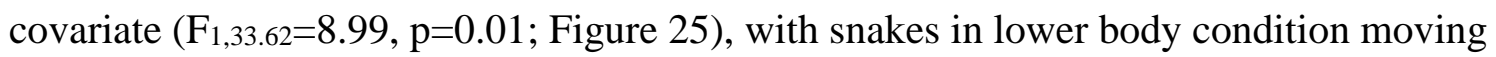
greater total distances during both periods. There was a trend for a body condition by sampling period interaction $\left(\mathrm{F}_{1,19.25}=3.88, \mathrm{p}=0.07\right)$, with snakes in the second period not influenced by body condition, and snakes in the first period influenced as described above. There was no effect of treatment and no interaction between treatment and period. Due to some snakes being unavailable for sampling, two snakes during time period 2 lacked a body condition measurement, and these data points were excluded from this analysis. Significance of other variables did not change when body condition was left out of the model and the two snake data points were included.

\section{Home range size}

Snakes had larger home ranges during the first period after implant treatment compared to period $2\left(\mathrm{~F}_{1,25.18}=5.78, \mathrm{p}=0.02\right)$ regardless of treatment (Figure 24B). There was no treatment by time period interaction. Due to some snakes being unavailable for 
sampling, two snakes during period 2 lacked a body condition measurement and were excluded from this analysis. We ran models with and without body condition as a covariate, and significance did not change, so ultimately report the model including body condition.

\section{Detectability}

There was a significant main effect of period $\left(\mathrm{F}_{1,25}=16.54, \mathrm{p}=0.0004\right.$; Figure 24C), with all snakes almost twice as likely to be detected in earlier period. There was no interaction between treatment and period and no main effect of treatment on detectability.

\section{Thermal Ecology}

Tb by period

Minimum, average, and range in $\mathrm{T}_{\mathrm{b}}$ all showed significant main effects of period $\left(F_{2,46.1}=6.10, p=0.005 ; F_{2,46.39}=11.3726, p<0.0001 ; F_{2,44.36}=5.4355, p=0.008 ;\right.$ Figure 26). Tukey's post-hoc tests revealed that snakes had lower minimum and average $\mathrm{T}_{\mathrm{b}}$ during the pre-implant period compared to post-implant period 2, and a larger range in $\mathrm{T}_{\mathrm{b}}$ during the pre-implant period compared to both post-implant periods. None of the predictors or covariates included in the model predicting maximum $T_{b}$ were significant. There were no significant main effects of treatment or treatment by period interactions in the $\mathrm{T}_{\mathrm{b}}$ by period models.

\section{Hourly fluctuation by period}

To better understand thermoregulatory ability, we modeled average fluctuation in body temperature by hour using sine and cosine to model circular movement of body temperature over a 24 hour period. There was a significant overall main effect of time of day (sine: $F_{1,1717}=4060.53$, $p<0.0001$; cosine: $F_{1,1717}=821.47, p<0.0001$ ). $\mathrm{T}_{b}$ was higher in 
the late afternoon and lowest in the early morning, as expected with an ectotherm conforming to environmental temperatures. There was an interaction between period and time of day (sine: $\mathrm{F}_{2,1717}=11.77$, $\mathrm{p}<0.0001$; cosine: $\mathrm{F}_{2,1717}=55.28$, $\mathrm{p}<0.0001$ ), driven by a significant main effect of period $\left(\mathrm{F}_{2,67}=14.56, \mathrm{p}<0.0001\right)$. Tukey's post hoc test revealed snakes had lower mean $T_{b}$ during the pre-implant period. There was no treatment by time of day interaction, nor was there a treatment by period interaction or main effect of treatment.

To understand fine-scale changes over the 24 hour period, the model above was run with hour of the day as a categorical variable in place of the sine and cosine variables. Again, time of day (hour), period, and period by time of day interaction were the only significant variables $\left(\mathrm{F}_{23,1633}=276.94, \mathrm{p}<0.0001 ; \mathrm{F}_{2,67}=14.53, \mathrm{p}<0.0001 ; \mathrm{F}_{46,1633}=4.65\right.$, $\mathrm{p}<0.0001)$. Highest $\mathrm{T}_{\mathrm{b}}$ were between 15:00-17:00, and coolest body temperatures were between 06:00-07:00 (Figure 27). In the pre-implant period, both treatment and control snakes had significantly lower body temperatures than period 1 for hours 21:00 and 0:002:00, and period 2 for hours 1:00-10:00, 19:00, and 21:00-23:00. Periods 1 and 2 were not significantly different for any body temperature. There were no significant differences between periods for body temperatures reached during 11:00 to 18:00, indicating that snakes were able to reach the same maximum $T_{b}$ regardless of period.

\section{Hourly temperature fluctuation by week}

To look at finer scale differences that may be due to effect of CORT release on a timescale different than detectable in 15 day periods, we modeled average hourly body temperature by week. There was a significant interaction between treatment and week

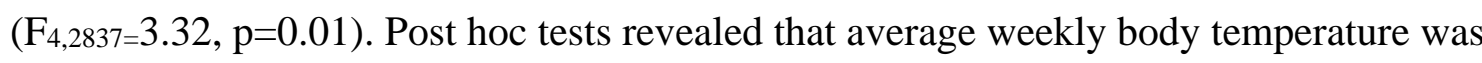


significantly different in the two treatment groups at the pre-implant and weeks 2 and 3, but not during weeks 1 or 4 . The week before implantation, treatment snakes had significantly higher body temperatures than control snakes, but after implant treatment the direction of effect was reversed; treatment snakes had lower body temperatures than controls in both weeks 2 and 3 (Figure 28). There was no main effect of treatment. There was a significant interaction between treatment and time, but only in the cosine time variable (sine: $\mathrm{F}_{1,2836}=0.70, \mathrm{p}=0.40$; cosine: $\mathrm{F}_{1,2836}=0.037, \mathrm{p}=0.037$ ). Time of day was

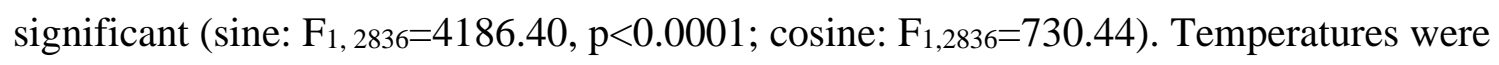
higher in the late afternoon and lowest in the early morning, as in the fluctuation in $\mathrm{T} b$. by period model. There was an interaction between week and time of day (sine:

$\mathrm{F} 4,2836=49.7331, \mathrm{p}<0.0001$; cosine: $\mathrm{F} 4,2836=25.2516, \mathrm{p}<0.0001)$, driven by a significant main effect of week $(F 14,2837=250.5771, \mathrm{p}<0.001)$.

Due to the small effect size differences in week 2 and 3 based on treatment group, we analyzed these weeks separately from the others, replacing sine and cosine of time with the categorical variable "hour" to elucidate if the snakes had an overall average difference in body temperature or if this was driven by maximum or minimum temperature differences at certain hours. There was no treatment by hour interaction detected, nor a main effect of treatment, so these models are not reported.

\section{Evaluating long term effects of CORT implants}

Spatial ecology

To determine long-term effects after a period of CORT elevation, home range sizes of snakes were analyzed post-implantation from late May 2015 to October 2015. 
These analyses exclude two snakes which disappeared or were depredated after month 1 in the study. Using both $95 \%$ and $100 \%$ MCPs, there was no effect of treatment (Figure A36-37). Initial body condition was a significant covariate in the $95 \% \mathrm{MCP}$ model only $\left(\mathrm{F}_{1,19}=5.83, \mathrm{p}=0.03\right.$; Figure 29$)$, with snakes in lower initial body condition having larger home ranges over the course of the study.

\section{Body condition}

Snakes decreased in body condition from the beginning of the study $\left(\mathrm{T}_{1,16}=-6.8\right.$, $\mathrm{p}<0.0001$; Figure 30), but there was no effect of treatment in the one-way ANOVA. SVL was marginally non-significant $\left(\mathrm{F}_{1,13}=4.3461, \mathrm{p}=0.0574\right)$ with smaller snakes showing a greater decrease in body condition over the study period (Figure 31).

\section{DISCUSSION}

\section{CORT implants elevated CORT in treatment snakes at sample 2}

CORT implant treatment in rattlesnakes elevated baseline plasma CORT levels in treatment snakes at the second sampling event, roughly 15 days after implantation. The magnitude of elevated baseline CORT in the treatment snakes at sample 2 falls within previously reported baseline levels of free-ranging rattlesnakes, including the sister species C. oreganus (Holding et al. 2014; Schuett et al. 2004; Lutterschmidt et al. 2009, Lind et al. 2010). Compared to acute elevation during the stress series, CORT was elevated to a much smaller degree via implants. However, chronically elevated CORT may have different effects than acute elevation, and may also affect change at different threshold levels (Dhabhar 2009; Seddon \& Klukowski 2012). Considering that CORT 
varies within individuals, populations, and across seasons in the aforementioned studies, we must evaluate "chronic stress" in the context of our study population. Therefore, we consider treatment snakes "chronically stressed" relative to the control group, at least for an average of 15 days post-implantation.

Ours is the first study to report experimentally elevated CORT via silastic implants for up to 15 days in a snake. Although we did not assess CORT levels between pre-implant and sample 2, there is a strong likelihood that plasma CORT between the samples was elevated to higher levels in treated snakes than detected at the sample 2, based on avian responses to CORT-implantation (Müller et al. 2009; Romero, Strochlic \& Wingfield 2005; DesRochers et al. 2009). This means that the snakes in our study may have experienced higher CORT levels that the modest increase in CORT we observed at sample 2. There is variation within the efficacy and duration of CORT-release from implants in both avian (Spee et al. 2011; Salvante \& Williams 2003; Müller et al. 2009; Romero, Strochlic \& Wingfield 2005; DesRochers et al. 2009) and reptilian studies (DeNardo \& Sinervo 1994; Miles et al. 2007; Juneau et al. 2015), with a range in effects from ten days to three months in reptiles (DeNardo \& Licht 1993; Cash \& Holberton 1999). We have shown it is possible to elevate baseline CORT via implants in rattlesnakes, although future studies may consider using a higher dosage to achieve higher CORT or re-implantation after 15 days to evaluate effects of chronically elevated CORT at a longer time scale.

Many studies emphasize the importance of drawing blood within a few minutes to ensure a proper baseline sample before a stress response is mounted (Romero \& Reed 2005), and must truncate the dataset due to observed significant relationships with CORT 
and time-to-bleed (Dunlap \& Wingfield 1995; Holding et al. 2014a; Sparkman et al. 2014). Sometimes, as in this study, no relationship between time-to-bleed and CORT is observed (Holding et al, 2014b; Hopkins \& DuRant 2011), which is likely because samples were collected within an adequate timeframe before circulating CORT increased due to stress response to handling (Schuett et al. 2004).

\section{Stress reactivity}

Stress reactivity was not affected by CORT implant treatment in rattlesnakes, but there was a trend for snakes with lower baseline CORT to mount larger stress responses. While we elevated CORT at sample 2 in treatment snakes, baseline CORT had decreased to control levels by sample 3 , when we conducted the stress reactivity assay. While we anticipated long-term effects on the HPA-axis after chronic CORT elevation, we did not detect any differences in stress response based on treatment group. This adds to the complicated literature assessing stress responses of chronically stressed animals (Jessop, Woodford \& Symonds 2013). In garter snakes, eleven days of multiple acute stressor exposure resulted in an elevated stress response (Neuman-Lee et al. 2015), while this led to a dampened stress response in European starlings (Rich and Romero 2005), even though baseline CORT levels in both studies were not different between 'stressed' animals and controls. Unlike the aforementioned studies, the stress response observed in this study was the similar for both CORT-treated and control snakes. It is possible that had we performed the stress reactivity assay at sample 2, when baseline CORT was higher in the treated snakes, we would have observed a difference between groups, which emphasizes the importance of investigating changes in both baseline and stressed CORT at many time points rather than at one final sample. 
We observed that all snakes reached similar levels of acute stressed CORT regardless of baseline levels, but that the magnitude of the response tended to be greatest in snakes with lower baseline CORT. Generally, higher baseline CORT is associated with higher stressed CORT (Dupoué et al. 2013; Sykes \& Klukowski 2009), although the magnitude of the response varies among species (reviewed in Cockrem 2013). At very high baseline levels, it is possible that no detectable stress response is mounted (Moore, Greene \& Mason 2001). To our knowledge, this is the first report of lower baseline CORT trending for a higher magnitude of stress response. Given that all snakes reached similar stressed CORT levels, perhaps this result indicates that rattlesnakes have a threshold range of stressed CORT that is reached from any baseline value after exposure to acute confinement stress.

We found a trend for snakes with greater increases in $T_{b}$ during the acute stress series to mount higher magnitude stress responses. This trend is well supported in the literature, as secretion of CORT by ectotherms is correlated with metabolic rate (Landys et al. 2006) and this secretion increases with temperature in many reptiles (Cree et al. 2003; Tyrrell \& Cree 1998; Jones \& Bell 2004; Romero \& Wikelski 2006). In some snakes, CORT release appears to be thermally independent (Sykes \& Klukowski 2009) or is increased at colder $\mathrm{T}_{\mathrm{b}}$ (Dupoué et al. 2013), but this does not appear to be the case in $C$. helleri. Our study provides some evidence for the thermal dependence of CORT secretion in rattlesnakes responding to acute confinement stress.

\section{Testosterone}

While the classic assumption is that stress exerts negative effects on reproduction (Wingfield \& Sapolsky 2003, Jones \& Bell 2004), it is not a hard-and-fast rule (Dayger et 
al. 2013; Narayan and Hero 2013). Baseline CORT was negatively correlated with testosterone in male Timber Rattlesnakes (Crotalus horridus) (Lutterschmidt et al. 2009), but acute stressed CORT led to increased testosterone. In a different population of the same species, no relationship between CORT and testosterone was observed (Lind \& Beaupre 2015). Contrasting relationships between CORT and testosterone are also observed in different populations of the garter snake Thamnophis sirtalis (Moore, Lemaster \& Mason 2000; Moore et al. 2000). We found no relationship between testosterone and treatment or CORT levels, which is supported by many other studies on rattlesnakes (Taylor, DeNardo \& Jennings 2004; Lind et al. 2010; Holding et al. 2014a; Lind \& Beaupre 2015) and other reptiles (Cree, Amey \& Whittier 2000; Moore \& Mason 2001). Our study was conducted shortly after the active breeding season for C. oreganus (Lind et al. 2010), which is likely similar for northern populations of C. helleri. As the relationship between CORT and testosterone can change based on reproductive season in some reptiles (Moore, Greene \& Mason 2001; Berger et al. 2005), repeating this study with implants administered during different seasons would be an important step in understanding if CORT and testosterone are independent, or if this relationship is seasondependent in this population.

Testosterone decreased in both treatment and control rattlesnakes approaching nightfall. This is a phenomenon observed in avian reptiles (Kempenaers, Peters \& Foerster 2008), but to our knowledge has not been reported in non-avian reptiles. One explanation for this phenomenon is that increased CORT during the morning takes several hours to show effects on testosterone suppression (Kempenaers, Peters \& Foerster 2008). However, we did not observe a time-of-day effect with CORT or a 
significant relationship between CORT and testosterone, which is expected if CORT was truly affecting changes in testosterone. It appears that diel cycling of testosterone is regulated by some other mechanism in these rattlesnakes, which warrants further investigation.

\section{Osmolality}

While osmolality was expected to increase in snakes with elevated CORT, no effect of treatment was observed. CORT can cross-react with aldosterone receptors, leading to fluid retention via increased solutes in the blood plasma (Thurnhorst, Beltz \& Johnson, 2007; Gaeggeler et al. 2005; Morris et al. 2000). It is possible that while CORT was elevated at sample 2, this level of CORT was not high enough to cause increased interaction with mineralocorticoid receptors, thus no effect on osmolality was observed. We did observe an overall effect of time on osmolality, however. At sample 3, snakes were more hydrated than at the pre-implant sample. As there was no rainfall or predation attempts during the sampling period, the most likely explanation for the observed hydration is in response to repeated sampling. We depleted venom from snakes at each sampling event for a separate study (see Chapter 2 of this thesis). Depleting fluids from the body from both venom and blood may have elicited a response for fluid retention via arginine vasotocin (AVT) or another factor leading to an observed decrease in blood osmolality (reviewed in Bradshaw 2007). However, snakes must have ingested water for this to be the case. Snakes did not have obvious food boluses during the post-implant periods, indicating food was not likely the source. Some snake home ranges overlapped with a small pond on the site, but many never accessed this area during the study. On June 9th, during post-implant period 1 , the field site received 0.25 inches of rain, and one 
snake was observed drinking droplets that accumulated on its head. Due to the lack of rain during the other days, we find it most likely that snakes were increasing osmolality by drinking dew droplets from the marine fog layer, which reliably covered the field site in the evening and receded by mid-morning. Many arid-adapted species drink dew formed from fog (Chouhan \& Sharma 2009) On several occasions, we witnessed snakes resting on the surface on cool mornings that would have had access to the droplets. We did not quantify levels of AVT or aldosterone in this study, but it may be important to account for in future repeated sampling studies conducted in arid environments.

\section{Defensive behavior}

Defensive behavior during tracking and sampling was not affected by treatment or baseline CORT, but defensive behavior at sampling was correlated to baseline T levels. While several studies have examined relationships between CORT and aggressive behavior (DeNardo \& Sinervo 1994; Yang \& Wilcynski 2003; Baird, Lovern \& Shine 2014), there have been surprisingly few studies investigating the relationship between stress and defensive behavior in terrestrial ectotherms, despite the common assumption that greater defensive behavior is exhibited by a 'stressed' animal. One study assessed the propensity of brown tree snakes (Boiga irregularis) to strike and found that strike frequency increased in sites with frequent and long-term survey activity (Spencer et al. 2015), which may be related to stress or baseline CORT levels. We found no effect of elevated CORT on defensive behavior in rattlesnakes, indicating that CORT does not appear to influence defensive behavior in this species. We found that snakes with higher testosterone, however, were likely to exhibit more defensive behaviors during the sampling period. The opposite effect was observed in garter snakes implanted with $\mathrm{T}$ 
(King 2001), indicating that effect of testosterone on defensive behavior may differ by species or general activity levels. Elevated testosterone is implicated in aggressive behaviors displayed between conspecifics in many reptiles during breeding contests or territorial disputes (Moore 1987; Kabelik, Weiss \& Moore 2006; While et al. 2010; Golinski et al. 2014), but does not necessarily predict the outcome of the contest in pitvipers (Schuett et al. 1996). The effects of testosterone on defensive behavior have received less attention, though our results suggest testosterone levels are important to consider in future defensive behavior studies.

During tracking periods, snakes with lower daily range in body temperature showed greater defensive behavior, but no effect of temperature on defensive behavior was observed during sampling. The temperature at sampling gives a better picture of the direct relationship between $T_{b}$ and behavior, while the $T_{b}$ across each period represents a coarser measurement comprised of daily temperature averages. Ectotherms' metabolic rate and thus other processes are strongly correlated to $\mathrm{T}_{b}$ (Lillywhite 1987), thus $\mathrm{Tb}_{\mathrm{b}}$ at sampling is a more direct indicator of effects on defensive behavior. In active-foraging reptiles, propensity to flee is correlated to increased $T_{b}$, while lower $T_{b}$ led to defense (Fitch 1965; Hertz et al. 1982; Arnold \& Bennet 1984; Bauder et al. 2015; but see Keogh \& DeSerto 1994). Based on ours and other studies, sit-and-wait foragers do not appear to show a temperature-dependent relationship with defensive behavior (Goode \& Duvall 1988; Gibbons \& Dorcas 2002). However, when viperid snakes are exposed or moving from one location to the next, they are more likely to flee (Maritz 2012) or exhibit active defensive behavior (Glaudas, Farrell \& May 2005). We did not observe snakes moving or elongated at a high enough incidence rate to allow investigation into this hypothesis. It is 
likely that even when warm, rattlesnakes and other viperids are too slow to mount an escape from a predator (Goode \& Duvall 1988), thus these types of snakes would benefit from relying on crypsis (Cooper, Caldwell, \& Vitt 2008; Maritz 2012) instead of drawing the attention of a predator with active defense (Hayes et al. 2002).

\section{Spatial ecology}

Total distance moved, home range size, and detectability of all snakes was greater in period 1 than period 2. Although CORT was elevated in treatment snakes during period 1, no differences were observed between treatment groups in spatial ecology parameters. We expected greater movement in the treatment group during period 1, because CORT mobilizes energy stores (Busch \& Hayward 2009), and is associated with dispersal and migration in many species (Wada 2008; Hamann, Jessop \& Schäuble 2007). CORT increases locomotor activity in captive lizards and turtles (Belliure \& Clobert 2004; Cash \& Holberton 1999), but to our knowledge, total distance moved and home range size in response to elevated CORT in terrestrial ectotherms had not been assessed until now. We did not detect a difference in total distance moved, home range size, or detectability based on treatment, indicating that rattlesnake spatial ecology is not greatly influenced by CORT.

In this study, all animals were outfitted with radiotransmitters, so the snakes were almost always 'detectable' in that they could be re-located. We were interested in the visual detectability of snakes, which relates to their use of the immediate habitat. Snakes under dense cover, in woodrat nests, or in burrows were not visually detectable. We interpret undetectable snakes as not foraging, prioritizing a stable thermal environment, or avoiding exposure to predators. Snakes in period 1 were more detectable, and also 
moved more and covered more ground. As all three of these variables follow the same time-dependent pattern, it is possible that the reproductive season for this population ended during our sampling period. Male rattlesnakes move more during the reproductive season in search of mates (Klauber 1972; Duvall \& Schuett 1997; Putman, Lind \& Taylor 2013) and have larger home ranges to maximize the chance of finding a mate. During these movements, it is likely the snakes encounter unfamiliar habitat and may be detected on the surface more often. The reproductive season may be taxing on snakes for this reason, leading to snakes settling in one spot to forage and replenish lost energy stores after the season ends. Greater movement is not necessarily always correlated to a greater home range size, as an animal could make many movements between two locations. In this case, snakes that moved more also covered greater area. Lower body condition snakes were associated with more movements, but not over a greater area- so low body condition snakes appear to be moving back and forth between a few locations, perhaps trying to maximize a foraging strategy (Wasko \& Sasa 2009).

\section{Body temperature $\left(T_{b}\right)$ and thermoregulation}

We detected an effect of treatment on average $T_{b}$ when analyzed by week. During the pre-implant period, treatment snakes held significantly higher average $T_{b}$ than controls, despite random assignment snakes to treatment groups. It is possible that due to the small sample size, this difference was driven by a few individuals. Although treatment snakes maintained higher $\mathrm{T}_{\mathrm{b}}$ than controls before implantation, after 2 and 3 weeks of implant treatment snakes had significantly lower $T_{b}$ than controls. While it is unclear why this effect was not observed during week 1 of implants, the switch in directionality shows that sustained, elevated CORT led to lower average $\mathrm{T}_{\mathrm{b}}$ in CORT- 
treated rattlesnakes. We predicted that snakes would maintain higher $\mathrm{T}_{\mathrm{b}}$ during elevated CORT, because elevated $T_{b}$ would drive an increased metabolic rate and thus increase the rate of energy mobilized (Landys et al. 2006), sustaining a snake through a search for better resources (Wingfield et al. 1998; Busch \& Hayward 2009). Several studies have reported positive correlations between CORT and $\mathrm{T}_{\mathrm{b}}$ in reptiles (Tyrrell and Cree 1998; Jessop et al. 2000; Cree et al. 2003; Woodley et al. 2003) but due to the relationship between $\mathrm{T}_{\mathrm{b}}$, metabolic rate, and CORT secretion (Landys et al. 2006), it is unclear in these studies whether CORT is driving $\mathrm{T}_{\mathrm{b}}$ through effects on behavioral thermoregulation or whether $\mathrm{T}_{\mathrm{b}}$ drives CORT secretion through its effects on metabolic rate. Exogenous CORT treatment has revealed that in female geckos (Hoplodactylus maculatus), heat seeking behavior is increased following treatment (Preest \& Cree 2008), while in wall lizards (Podarcis muralis) heat avoidance behavior is observed (Belliure \& Clobert 2004). In support of our result, a study on translocated rattlesnakes reported lower mean $\mathrm{T}_{\mathrm{b}}$ during the week immediately after translocation than controls (Holding, Owen \& Taylor 2014), which may be related to differences in circulating CORT, although this was not directly assessed (Holding et al. 2014a). Our treatment of CORT in rattlesnakes led to lower $\mathrm{T}_{\mathrm{b}}$, suggesting that elevated CORT impacts thermoregulatory behavior and not the reverse, as all snakes experienced similar ambient temperature regimes. This may indicate that rattlesnakes actively resist the effects of elevated CORT at higher metabolic rates by choosing to operate at lower $\mathrm{T}_{\mathrm{b}}$. When rattlesnakes enter a state of chronic stress, conditions are likely inescapable and do not improve for a long duration of time (i.e. drought, no food available), so snakes that operate at lower $\mathrm{Tb}$ and thus lower metabolisms could prioritize survival by metabolizing energy stores at a lower rate 
(Landys et al. 2006). This trait could eventually be selected for in a population of rattlesnakes that experiences frequent drought and famine. It is important to note, however, that we did not observe thermoregulatory behavior or thermal preferences directly, and this hypothesis deserves further study and attention in a carefully designed thermal preference experiment.

We observed hourly temperature differences in all snakes, with higher $\mathrm{T}_{\mathrm{b}}$ in the late afternoon (15:00-17:00) and lowest $\mathrm{T}_{\mathrm{b}}$ in the early morning hours (04:00-08:00). This is typical for a ectotherm that conforms to the temperature of its environment (Seebacher 2005). Snakes in the pre-implant period had significantly lower $T_{b}$ during the morning hours than periods 1 and 2, which is expected as the ambient temperature increased between each period, approaching mid-summer. Interestingly, $\mathrm{T}_{\mathrm{b}}$ in the afternoon was not different between periods, indicating that snakes were able to achieve the similar $\mathrm{T}_{\mathrm{b}}$ by basking during all periods, regardless of morning $\mathrm{T}_{\mathrm{b}} \mathrm{s}$. The observed afternoon high $\mathrm{T}_{\mathrm{b}}$ may also represent preferred body temperature for this species, although thermal preferences were not directly assessed in this study. The average daily $\mathrm{T}_{\mathrm{b}}$ analysis of minimum, range, average and maximum $\mathrm{T}_{\mathrm{b}}$ for each period mirrors the results observed in the hourly analysis by period. Minimum and average $\mathrm{T}_{\mathrm{b}}$ were lower in the pre-implant period than post-implant period 2 , and range in $T_{b}$ was higher than the pre-implant period in post-implant periods 1 and 2 . $\mathrm{T}_{\mathrm{a}}$ at the field site was not significantly related to snake $\mathrm{T}_{\mathrm{b}}$, but this is likely due to snakes using habitat creating a different thermal environment from the ambient temperature, such as burrows, woodrat nests, concrete slabs, or dense vegetative cover. We did not account for fine-scale movements among thermal 
environments during the day, but all snakes were observed in some of these micro-habitat types throughout the study.

\section{Long term effects}

We did not detect long term effects of treatment on home range size or body condition. Chronic stress can alter behavior and physiology long after CORT has returned to baseline (Rich \& Romero 2005; Neuman-Lee et al. 2015). We predicted that a period of elevated CORT in rattlesnakes would result in overall decreased body condition, and that movement would increase due to the effect of CORT mobilizing energy stores (Wingfield et al. 1998; Busch \& Hayward 2009), and acting as a signal for dispersal and migration in some species (Hamann, Jessop \& Schäuble 2007; Wada 2008). In Western fence lizards, long-term elevated CORT decreased home range sizes if aggressive conspecifics were present (DeNardo \& Sinervo 1994). We did not detect long term effects of treatment in these parameters, although CORT was elevated for at least 15 days in treatment snakes. Again, it is possible that long term effects of CORT result from sustained levels of CORT higher than reached in this study. Despite this, we observed that all snakes decreased in body condition over the course of the study, and smaller snakes showed greater decreases. These effects could be attributed to repeated venom sampling preventing successful prey capture (see Chapter 2 of this thesis), leaving snakes essentially fasted during the study period. While possible effects of frequent tracking could have impacted snake foraging leading to decreased body condition, these impacts have been deemed negligible in another species of snake (Fauvel et al. 2012). 


\section{CONCLUSIONS}

To our knowledge, this is the first study to exogenously manipulate CORT via implants in snakes and to evaluate effects of elevated CORT over time in a wild population of snakes. We show that although baseline CORT was successfully elevated in treatment snakes, T levels, osmolality, stress reactivity, defensive behavior, and spatial ecology remain largely unaffected. We report an effect of treatment on thermoregulation, with treatment snakes having lower average $T_{b}$ than control snakes at weeks 2 and 3 postimplant. Whether this is a direct effect of CORT influencing thermoregulatory behavior and metabolism is unknown. No long-term effects of treatment were observed on body condition or home range size. We observed trends between baseline CORT levels and magnitude of stress response mounted. We also report correlations between T levels and defensive behavior, suggesting that $\mathrm{T}$ should be assessed in addition to CORT in future defensive behavior studies. Overall, rattlesnakes appear resistant to effects of elevated CORT. While we did not see effects of treatment on most parameters, it is important to note that raising CORT to higher levels and for a longer period may be necessary to better mimic chronic stress conditions and induce effects on the parameters we measured. It may be that for CORT to induce measurable effects in ectotherms with low metabolic rates, it must be elevated to levels much higher and longer than in endotherms. Our study provides evidence for stress-tolerance in rattlesnakes, a group of ectotherms that regularly tolerates periods of drought and low-food availability. Much remains to be studied about the effects of chronic stress on terrestrial ectotherm ecology. 


\section{TABLE}

Table 2. Results from various analyses. Significant factors and covariates are bolded

\begin{tabular}{|c|c|c|c|}
\hline Response & Factor & F-statistic & $p$-value \\
\hline \multirow{7}{*}{ Baseline CORT } & Treatment & $\mathrm{F}_{1,22.43}=3.58$ & $\mathrm{p}=0.07$ \\
\hline & Sample & $\mathrm{F}_{2,43.3}=1.34$ & $\mathrm{p}=0.27$ \\
\hline & Treatment*Sample & $F_{2,41.35}=3.33$ & $p=0.046$ \\
\hline & Time-to-bleed & $\mathrm{F}_{1,57.94}=0.28$ & $\mathrm{p}=0.60$ \\
\hline & Time of day & $\mathrm{F}_{1,57.77}=1.53$ & $\mathrm{p}=0.22$ \\
\hline & $\mathrm{T}_{\mathrm{b}}$ & $\mathrm{F}_{1,59.8}=1.03$ & $\mathrm{p}=0.31$ \\
\hline & SVL & $\mathrm{F}_{1,20.73}=1.42$ & $\mathrm{p}=0.25$ \\
\hline \multirow[t]{7}{*}{ Testosterone } & Treatment & $\mathrm{F}_{1,24.4}=0.16$ & $\mathrm{p}=0.69$ \\
\hline & Sample & $\mathrm{F}_{2,41.77}=0.86$ & $\mathrm{p}=0.43$ \\
\hline & Treatment*Sample & $F_{2,40.97}=2.24$ & $\mathrm{p}=0.12$ \\
\hline & Size Class & $F_{1,21.99}=8.64$ & $p=0.0076$ \\
\hline & SVL & $\mathrm{F}_{1,22.64}=1.35$ & $\mathrm{p}=0.26$ \\
\hline & $\mathrm{T}_{\mathrm{b}}$ & $\mathrm{F}_{1,53.77}=1.82$ & $\mathrm{p}=0.18$ \\
\hline & Time of day & $F_{1,46}=3.05$ & $\mathrm{p}=0.09$ \\
\hline \multirow[t]{8}{*}{ Osmolality } & Treatment & $\mathrm{F}_{1,23.39}=2.00$ & $\mathrm{p}=0.17$ \\
\hline & Sample & $F_{2,45.09}=8.16$ & $p=0.009$ \\
\hline & Treatment*Sample & $F_{2,40.72}=0.996$ & $\mathrm{p}=0.39$ \\
\hline & CORT & $F_{1,60}=0.46$ & $\mathrm{p}=0.50$ \\
\hline & Body condition & $\mathrm{F}_{1,47.79}=1.55$ & $\mathrm{p}=0.22$ \\
\hline & SVL & $\mathrm{F}_{1,19.62}=0.65$ & $\mathrm{p}=0.43$ \\
\hline & Time of Day & $\mathrm{F}_{1,57.75}=2.32$ & $\mathrm{p}=0.13$ \\
\hline & Time-to-bleed & $F_{1,56.65}=8.04$ & $p=0.006$ \\
\hline \multirow{6}{*}{$\begin{array}{l}\text { Acute Stressed } \\
\text { CORT }\end{array}$} & Treatment & $\mathrm{F}_{1,14.01}=0.01$ & $\mathrm{p}=0.91$ \\
\hline & Sample & $F_{1,19.69}=50.30$ & $p<0.0001$ \\
\hline & Treatment*Sample & $\mathrm{F}_{1,17.01}=0.01$ & $\mathrm{p}=0.91$ \\
\hline & Time of Day & $\mathrm{F}_{1,13.69}=0.91$ & $\mathrm{p}=0.36$ \\
\hline & Initial $\mathrm{T}_{\mathrm{b}}$ & $\mathrm{F}_{1,22.5}=0.62$ & $\mathrm{p}=0.44$ \\
\hline & Testosterone & $\mathrm{F}_{1,15.26}=0.30$ & $\mathrm{p}=0.60$ \\
\hline \multirow{3}{*}{$\begin{array}{l}\text { Acute Change in } \\
\text { CORT } \\
\text { Magnitude of Stress } \\
\text { Response }\end{array}$} & Treatment & $\mathrm{F}_{1,15}=0.01$ & $\mathrm{p}=0.91$ \\
\hline & Initial CORT & $\mathrm{F}_{1,15}=3.36$ & $\mathrm{p}=0.09$ \\
\hline & Change in $T_{b}$ & $\mathrm{~F}_{1,15}=2.30$ & $\mathrm{p}=0.09$ \\
\hline
\end{tabular}


Table 2 cont'd

\begin{tabular}{|c|c|c|c|}
\hline Response & Factor & F-statistic & $p$-value \\
\hline \multirow{6}{*}{$\begin{array}{l}\text { Defensive Behavior } \\
\text { Tracking period }\end{array}$} & Treatment & $\mathrm{F}_{1,18.47}=0.15$ & $\mathrm{p}=0.71$ \\
\hline & Period & $\mathrm{F}_{1,20.12}=1.02$ & $\mathrm{p}=0.32$ \\
\hline & Treatment*Period & $F_{1,20.26}=0.009$ & $\mathrm{p}=0.92$ \\
\hline & Change in CORT & $\mathrm{F}_{1,18.93}=1.4360$ & $\mathrm{p}=0.24$ \\
\hline & SVL & $\mathrm{F}_{1,16.36}=1.44$ & $\mathrm{p}=0.25$ \\
\hline & Range in $\mathbf{T}_{\mathbf{b}}$ & $F_{1,18.93}=5.20$ & $p=0.03$ \\
\hline \multirow{7}{*}{$\begin{array}{l}\text { Defensive Behavior } \\
\text { During Sampling }\end{array}$} & Treatment & $\mathrm{F}_{1,12.54}=1.41$ & $\mathrm{p}=0.26$ \\
\hline & Sample & $\mathrm{F}_{2,37.3}=0.26$ & $\mathrm{p}=0.78$ \\
\hline & Treatment*Sample & $\mathrm{F}_{2,37.59}=1.60$ & $\mathrm{p}=0.22$ \\
\hline & CORT & $\mathrm{F}_{1,52.99}=0.01$ & $\mathrm{p}=0.91$ \\
\hline & $\mathrm{T}_{\mathrm{b}}$ & $\mathrm{F}_{1,40.55}=0.73$ & $\mathrm{p}=0.40$ \\
\hline & SVL & $\mathrm{F}_{1,13.53}=1.27$ & $\mathrm{p}=0.28$ \\
\hline & Testosterone & $F_{1,26.86}=5.87$ & $p=0.02$ \\
\hline \multirow{4}{*}{$\begin{array}{l}\text { Total Distance Moved } \\
\text { Pre-Implant Period }\end{array}$} & Treatment & $\mathrm{F}_{1,20}=1.64$ & $\mathrm{p}=0.21$ \\
\hline & SVL & $\mathrm{F}_{1,20}=0.70$ & $\mathrm{p}=0.41$ \\
\hline & Body Condition & $\mathrm{F}_{1,20}=0.08$ & $\mathrm{p}=0.78$ \\
\hline & $\begin{array}{l}\text { Number of GPS } \\
\text { points }\end{array}$ & $\mathrm{F}_{1,20}=0.01$ & $\mathrm{p}=0.94$ \\
\hline \multirow{7}{*}{$\begin{array}{l}\text { Total Distance Moved } \\
\text { By Post-Implant Period }\end{array}$} & Treatment & $\mathrm{F}_{1,19.18}=0.09$ & $\mathrm{p}=0.77$ \\
\hline & Period & $F_{1,21.19}=7.96$ & $p=0.01$ \\
\hline & Treatment*Period & $\mathrm{F}_{1,17.76}=0.21$ & $\mathrm{p}=0.66$ \\
\hline & Body Condition & $F_{1,33.62}=8.99$ & $\mathrm{p}=0.01$ \\
\hline & Body Condn*Period & $\mathrm{F}_{1,19.25}=3.88$ & $\mathrm{p}=0.07$ \\
\hline & SVL & $\mathrm{F}_{1,19.03}=0.01$ & $\mathrm{p}=0.91$ \\
\hline & Number GPS Points & $\mathrm{F}_{1,24.73}=1.17$ & $\mathrm{p}=0.29$ \\
\hline \multirow{6}{*}{$\begin{array}{l}\text { Home Range Size } \\
\text { By Post-Implant Period }\end{array}$} & Treatment & $F_{1,19.99}=0.21$ & $\mathrm{p}=0.66$ \\
\hline & Period & $F_{1,25.18}=5.78$ & $p=0.02$ \\
\hline & Treatment*Period & $\mathrm{F}_{1,20.24}=0.96$ & $\mathrm{p}=0.34$ \\
\hline & SVL & $\mathrm{F}_{1,19.68}=0.08$ & $\mathrm{p}=0.78$ \\
\hline & Number GPS Points & $\mathrm{F}_{1,31.35}=0.45$ & $\mathrm{p}=0.51$ \\
\hline & Body Condition & $\mathrm{F}_{1,43.74}=1.72$ & $\mathrm{p}=0.20$ \\
\hline
\end{tabular}


Table 2 cont' $d$

\begin{tabular}{|c|c|c|c|}
\hline Response & Factor & F-statistic & $p$-value \\
\hline \multirow{5}{*}{$\begin{array}{l}\text { Detectability } \\
\text { By Post-Implant Period }\end{array}$} & Treatment & $\mathrm{F}_{1,23.4}=1.20$ & $\mathrm{p}=0.28$ \\
\hline & Period & $\mathrm{F}_{1,25}=16.54$ & $\mathrm{p}=0.0004$ \\
\hline & Treatment*Period & $\mathrm{F}_{1,24.2}=0.96$ & $\mathrm{p}=0.34$ \\
\hline & Number GPS Points & $\mathrm{F}_{1,29.66}=0.17$ & $\mathrm{p}=0.68$ \\
\hline & SVL & $\mathrm{F}_{1,23.55}=0.49$ & $\mathrm{p}=0.49$ \\
\hline \multirow{6}{*}{$\begin{array}{l}\text { Maximum } \mathbf{T}_{b} \\
\text { By Period }\end{array}$} & Treatment & $\mathrm{F}_{1,26.32}=0.01$ & $\mathrm{p}=0.92$ \\
\hline & Period & $\mathrm{F}_{2,52.86}=0.62$ & $\mathrm{p}=0.55$ \\
\hline & Treatment*Period & $\mathrm{F}_{2,44.68}=1.21$ & $\mathrm{p}=0.31$ \\
\hline & SVL & $\mathrm{F}_{1,20.83}=0.004$ & $\mathrm{p}=0.95$ \\
\hline & Body Condition & $\mathrm{F}_{1,34.03}=0.10$ & $\mathrm{p}=0.76$ \\
\hline & $\mathrm{T}_{\mathrm{a}}(\max )$ & $\mathrm{F}_{1,61.92}=1.20$ & $\mathrm{p}=0.28$ \\
\hline \multirow{6}{*}{$\begin{array}{l}\text { Minimum } \mathbf{T}_{\mathrm{b}} \\
\text { By Period }\end{array}$} & Treatment & $\mathrm{F}_{1,31.52}=0.40$ & $\mathrm{p}=0.53$ \\
\hline & Period & $F_{2,46.1}=6.10$ & $p=0.005$ \\
\hline & Treatment*Period & $\mathrm{F}_{2,42.93}=0.04$ & $\mathrm{p}=0.96$ \\
\hline & SVL & $\mathrm{F}_{1,21.3}=1.40$ & $\mathrm{p}=0.25$ \\
\hline & Body Condition & $F_{1,59.73}=0.16$ & $\mathrm{p}=0.69$ \\
\hline & $\mathrm{T}_{\mathrm{a}}(\min )$ & $\mathrm{F}_{1,48.77}=0.71$ & $\mathrm{p}=0.40$ \\
\hline \multirow{6}{*}{$\begin{array}{l}\text { Average } \mathbf{T}_{\mathrm{b}} \\
\text { By Period }\end{array}$} & Treatment & $\mathrm{F}_{1,31.25}=0.1718$ & $\mathrm{p}=0.6813$ \\
\hline & Period & $F_{2,46.39}=11.3726$ & $\mathrm{p}<0.0001$ \\
\hline & Treatment*Period & $\mathrm{F}_{2,43.13}=0.02$ & $\mathrm{p}=0.98$ \\
\hline & SVL & $\mathrm{F}_{1,21.38}=0.50$ & $\mathrm{p}=0.49$ \\
\hline & Body condition & $\mathrm{F}_{1,60.02}=0.14$ & $\mathrm{p}=0.71$ \\
\hline & $\mathrm{T}_{\mathrm{a}}(\min )$ & $\mathrm{F}_{1,49.26}=0.04$ & $\mathrm{p}=0.83$ \\
\hline \multirow{6}{*}{$\begin{array}{l}\text { Range in } T_{b} \\
\text { By Period }\end{array}$} & Treatment & $\mathrm{F}_{1,30.39}=0.1694$ & $\mathrm{p}=0.68$ \\
\hline & Period & $F_{2,44.36}=5.4355$ & $p=0.008$ \\
\hline & Treatment*Period & $\mathrm{F}_{2,43.23}=0.24$ & $\mathrm{p}=0.79$ \\
\hline & SVL & $\mathrm{F}_{1,21.52}=0.60$ & $\mathrm{p}=0.45$ \\
\hline & Body Condition & $\mathrm{F}_{1,61.08}=0.08$ & $\mathrm{p}=0.78$ \\
\hline & $\mathrm{T}_{\mathrm{a}}$ (range) & $\mathrm{F}_{1,45.88}=1.62$ & $\mathrm{p}=0.21$ \\
\hline
\end{tabular}




\begin{tabular}{|c|c|c|c|}
\hline Response & Factor & F-statistic & $p$-value \\
\hline \multirow{10}{*}{$\begin{array}{l}\text { Daily Fluctuation } \\
\text { By Period }\end{array}$} & Treatment & $F_{1,67}=0.0021$ & $\mathrm{p}=0.96$ \\
\hline & Period & $F_{2,67}=14.56$ & $p<0.0001$ \\
\hline & Treatment*Period & $\mathrm{F}_{2,67}=0.12$ & $\mathrm{p}=0.89$ \\
\hline & Sine time & $F_{1,1717}=4060.53$ & $p<0.0001$ \\
\hline & Cos time & $\mathbf{F}_{1,1717}=821.47$ & $p<0.0001$ \\
\hline & Treatment* sine time & $\mathrm{F}_{1,1717}=0.34$ & $\mathrm{p}=0.56$ \\
\hline & Treatment*cos time & $\mathrm{F}_{1,1717}=1.47$ & $\mathrm{p}=0.23$ \\
\hline & SVL & $F_{1,67}=1.60$ & $\mathrm{p}=0.21$ \\
\hline & Period*sine time & $F_{2,1717}=11.77$ & $\mathrm{p}<0.0001$ \\
\hline & Period*cos time & $F_{2,1717}=55.28$ & $p<0.0001$ \\
\hline \multirow{6}{*}{$\begin{array}{l}\text { Hourly Fluctuation } \\
\text { By Period }\end{array}$} & Treatment & $\mathrm{F}_{1,67}=0.01$ & $\mathrm{p}=0.92$ \\
\hline & Period & $\mathrm{F}_{2,67}=14.53$ & p $<0.0001$ \\
\hline & Treatment*Period & $\mathrm{F}_{2,67}=0.12$ & $\mathrm{p}=0.89$ \\
\hline & Hour & $\mathrm{F}_{23,1633}=276.94$ & $\mathrm{p}<0.0001$ \\
\hline & Hour*Period & $F_{46,1633}=4.65$ & p $<0.0001$ \\
\hline & SVL & $\mathrm{F}_{1,67}=1.45$ & $\mathrm{p}=0.23$ \\
\hline \multirow{7}{*}{$\begin{array}{l}\text { Hourly Fluctuation } \\
\text { by Week }\end{array}$} & Treatment & $\mathrm{F}_{1,20}=0.14$ & $\mathrm{p}=0.71$ \\
\hline & Week & $\mathrm{F}_{14,2837}=250.58$ & $\mathrm{p}<0.001$ \\
\hline & Treatment*Week & $\mathrm{F}_{4,2837=3.32}$ & $p=0.01$ \\
\hline & Cosine Time & $\mathrm{F}_{1,2836}=730.44$ & $\mathrm{p}<0.0001$ \\
\hline & Sine Time & $\mathrm{F}_{1,2836}=4186.40$ & $\mathrm{p}<0.0001$ \\
\hline & Week*Sine Time & $\mathrm{F}_{4,2836}=49.7331$ & $\mathrm{p}<0.0001$ \\
\hline & Week*Cos Time & $\mathrm{F}_{4,2836}=25.2516$ & $\mathrm{p}<0.0001$ \\
\hline \multirow{4}{*}{$\begin{array}{l}\text { Home Range Size } \\
\text { 100\% MCP, long term }\end{array}$} & Treatment & $\mathrm{F}_{1,19}=1.35$ & $\mathrm{p}=0.26$ \\
\hline & SVL & $\mathrm{F}_{1,19}=0.003$ & $\mathrm{p}=0.96$ \\
\hline & Number GPS points & $\mathrm{F}_{1,19}=1.99$ & $\mathrm{p}=0.18$ \\
\hline & Initial body cond. & $\mathrm{F}_{1,19}=2.05$ & $\mathrm{p}=0.17$ \\
\hline \multirow{4}{*}{$\begin{array}{l}\text { Home Range Size } \\
95 \% \text { MCP, long term }\end{array}$} & Treatment & $\mathrm{F}_{1,19}=0.17$ & $\mathrm{p}=0.68$ \\
\hline & SVL & $F_{1,19}=0.01$ & $\mathrm{p}=0.92$ \\
\hline & Number GPS points & $\mathrm{F}_{1,19}=3.00$ & $\mathrm{p}=0.10$ \\
\hline & Initial body cond & $F_{1,19}=5.83$ & $p=0.03$ \\
\hline Body condition & Treatment & $\mathrm{F}_{1,13}=1.6006$ & $\mathrm{p}=0.2280$ \\
\hline Change over entire study & SVL & $F_{1,13}=4.3461$ & $\mathrm{p}=0.0574$ \\
\hline
\end{tabular}




\section{FIGURES}

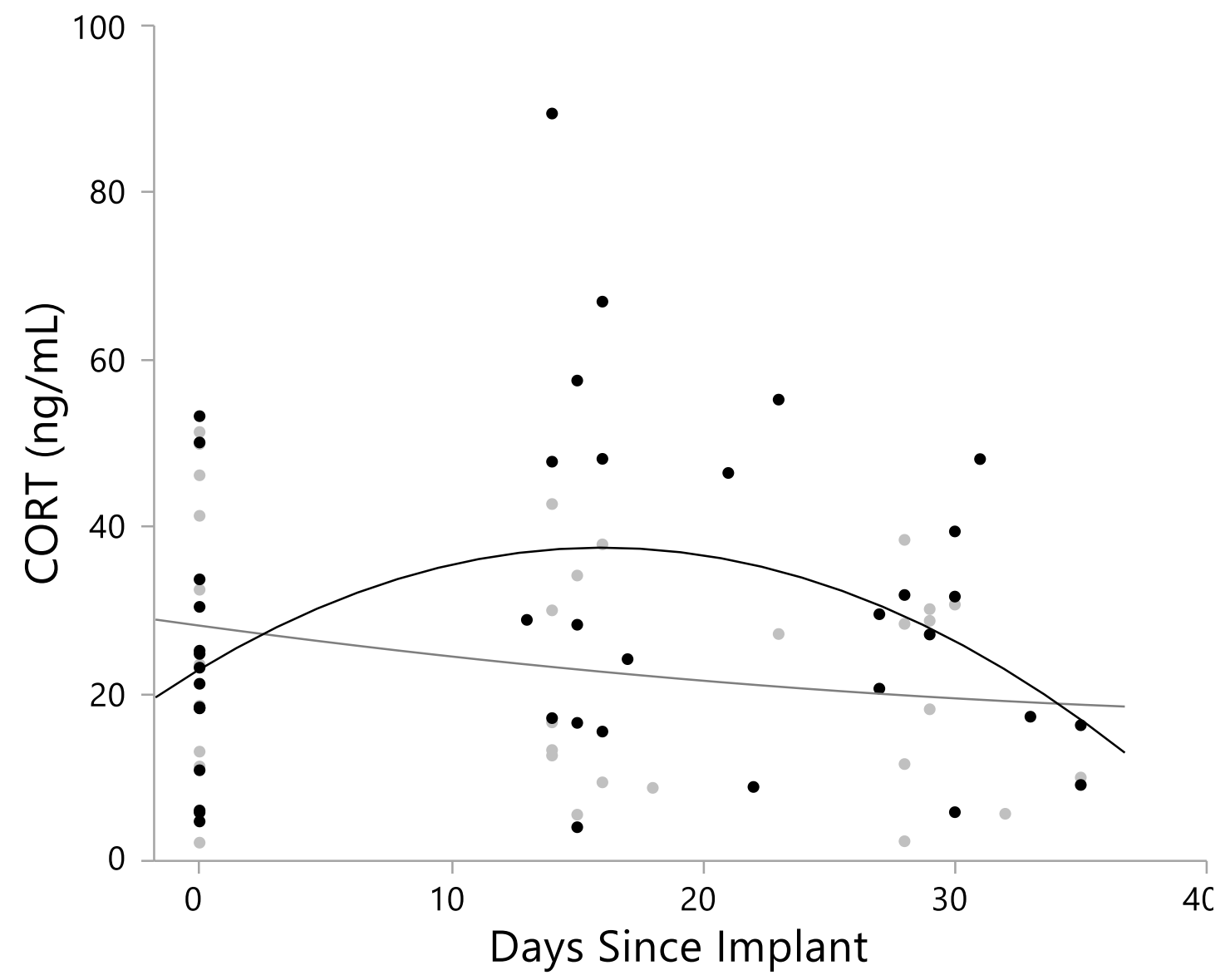

Figure 13. CORT plotted against days since implant administration $($ light $=$ control, dark $=$ CORT $)$ for Crotalus helleri. Sample 2 fell between days 12-22. Sample 3 fell between days 27-35. 


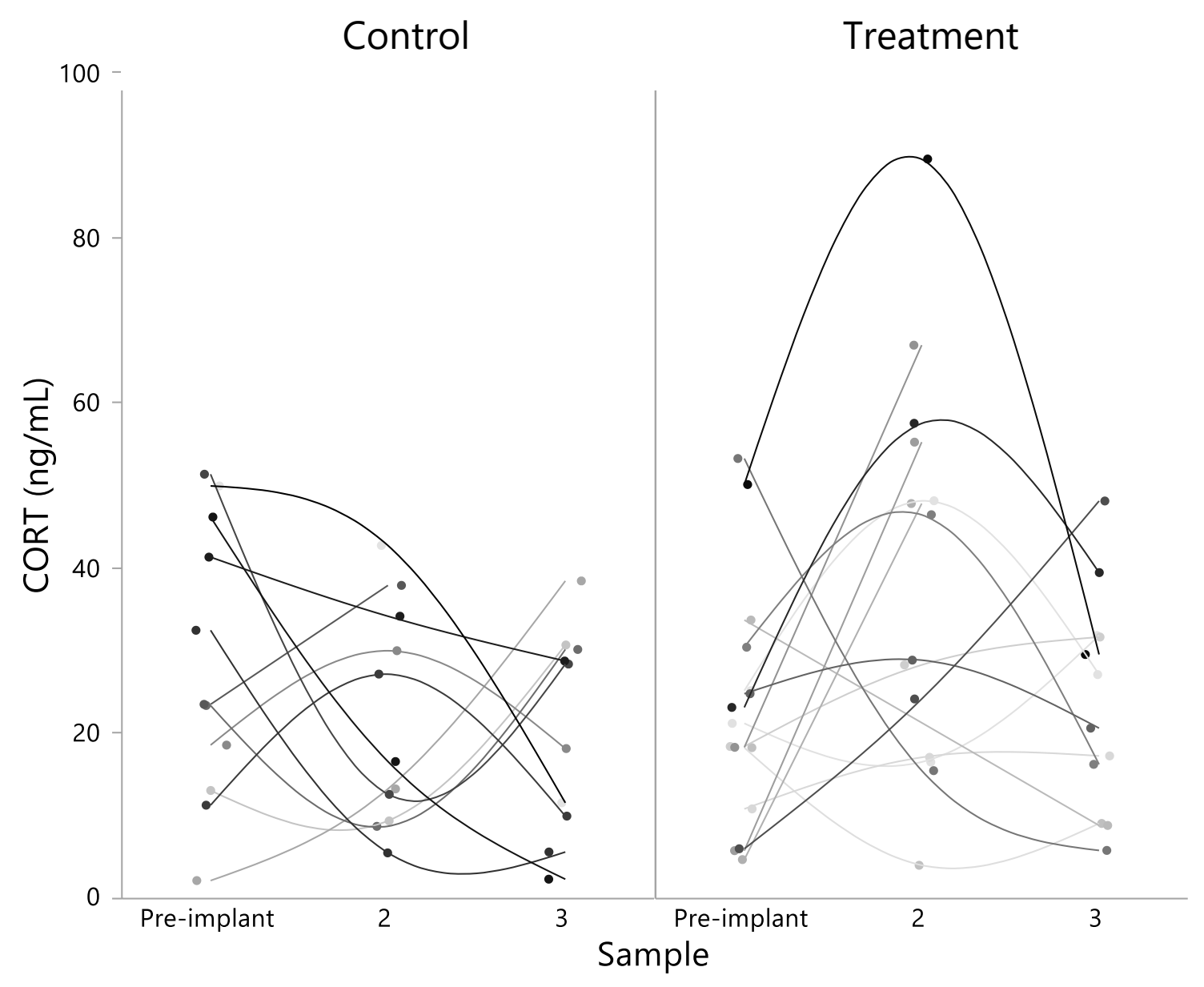

Figure 14. Individual CORT responses of Crotalus helleri to implantation of control (left) or CORT (right) implants across three samples. Each line represents one snake, while each point on the line represents a sampling event. Individual lines and points are shaded similar colors of gray. 


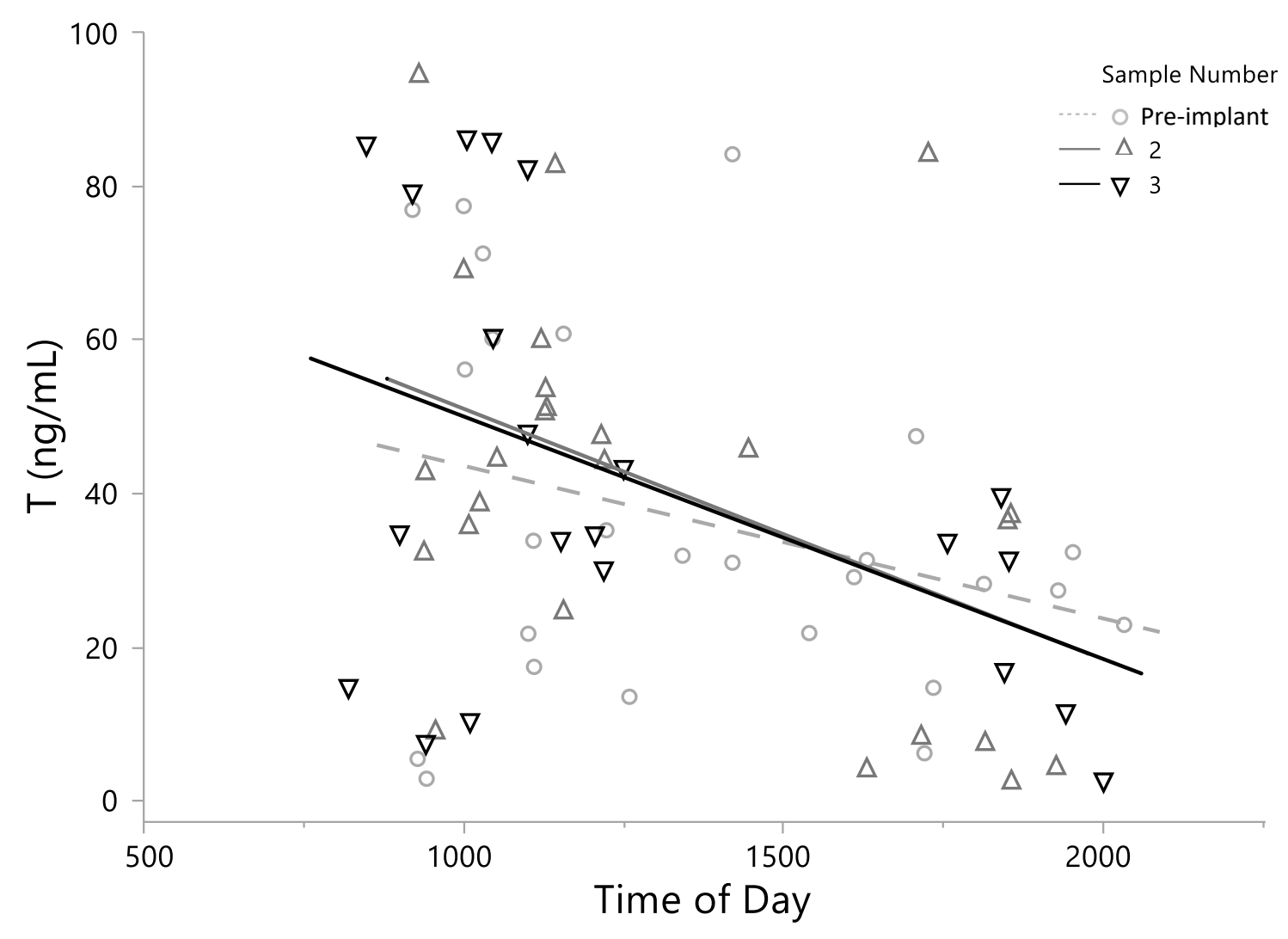

Figure 15. Plasma T plotted against time of day for Crotalus helleri at each sample (coded sequentially from light to dark). There was a trend for $\mathrm{T}$ to decrease late in the day. 


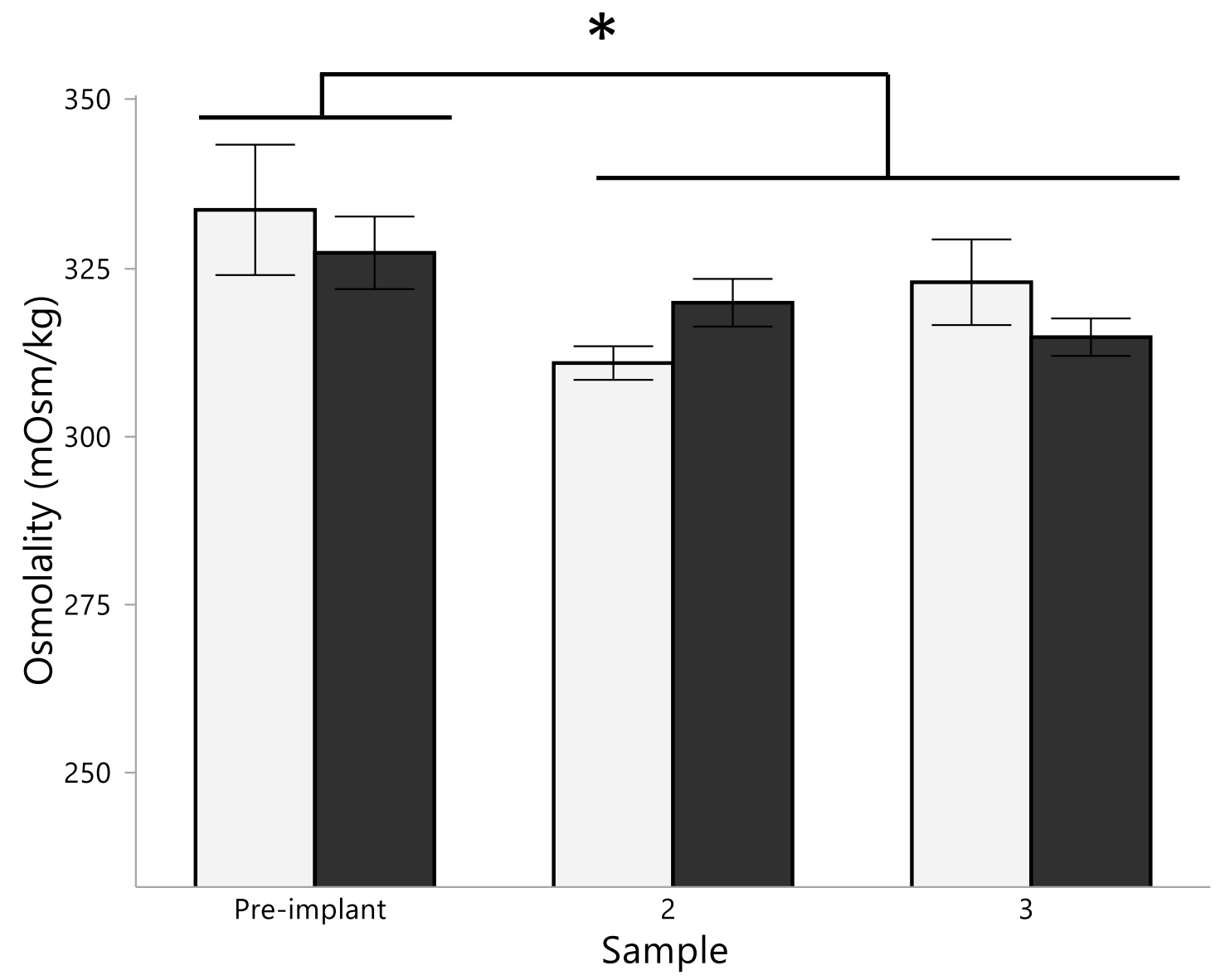

Figure 16. Plasma osmolality of treated (dark bars) and control (light bars) Crotalus helleri at each sampling period. Osmolality was not affected by treatment. Samples 2 and 3 for both groups had lower osmolality than the pre-implant samples. Groups marked with an asterisk are significantly different. Error bars are \pm 1 SEM. 


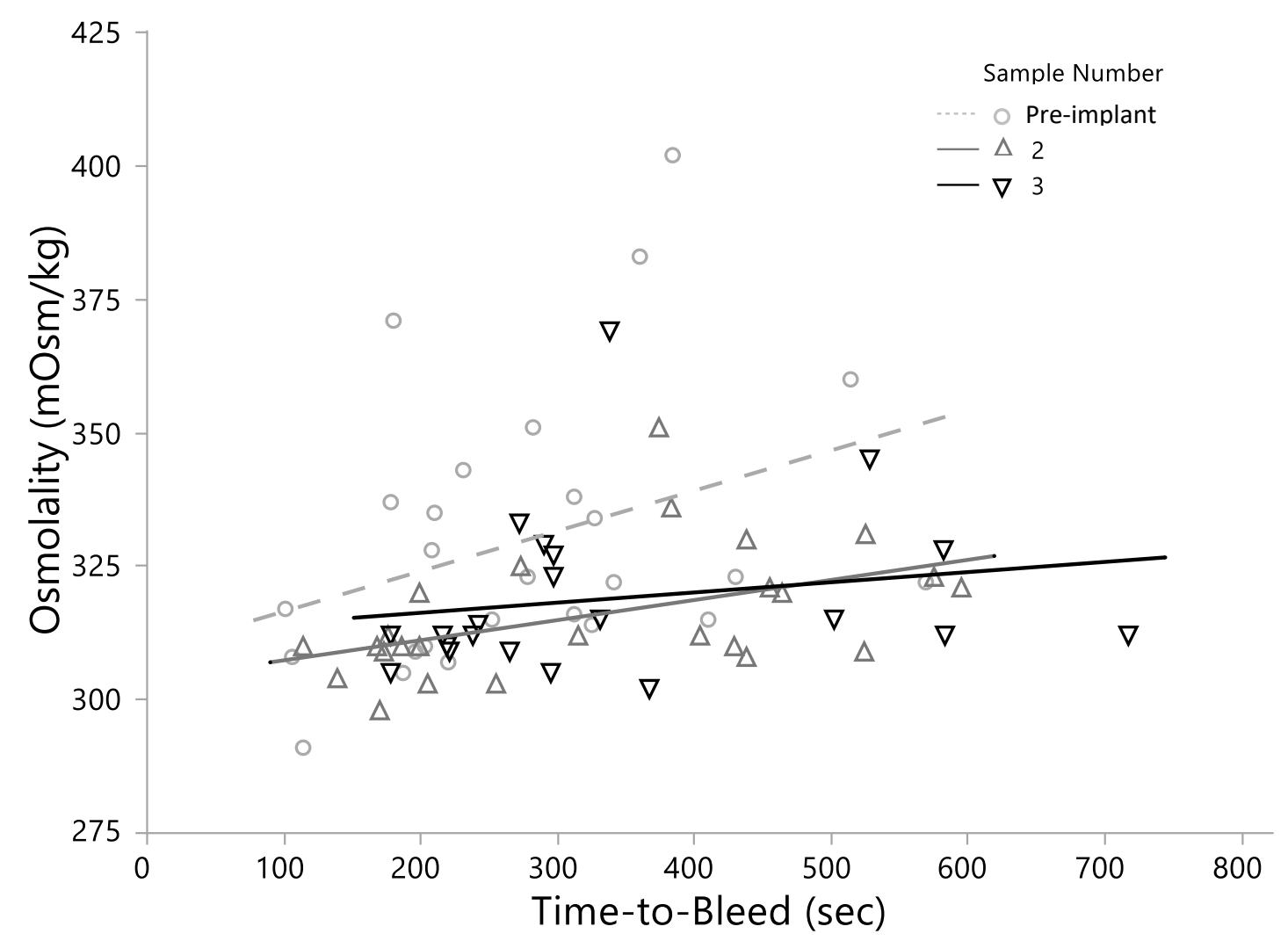

Figure 17. Plasma osmolality plotted against time-to-bleed across all three sampling periods (coded sequentially from light to dark). Time-to-bleed was a significant covariate in the RM ANCOVA. Although all three samples from each snake are shown, each within-snake sample was not considered independent. The RM ANCOVA accounted for repetition within individuals, so there was no pseudoreplication in our dataset. 


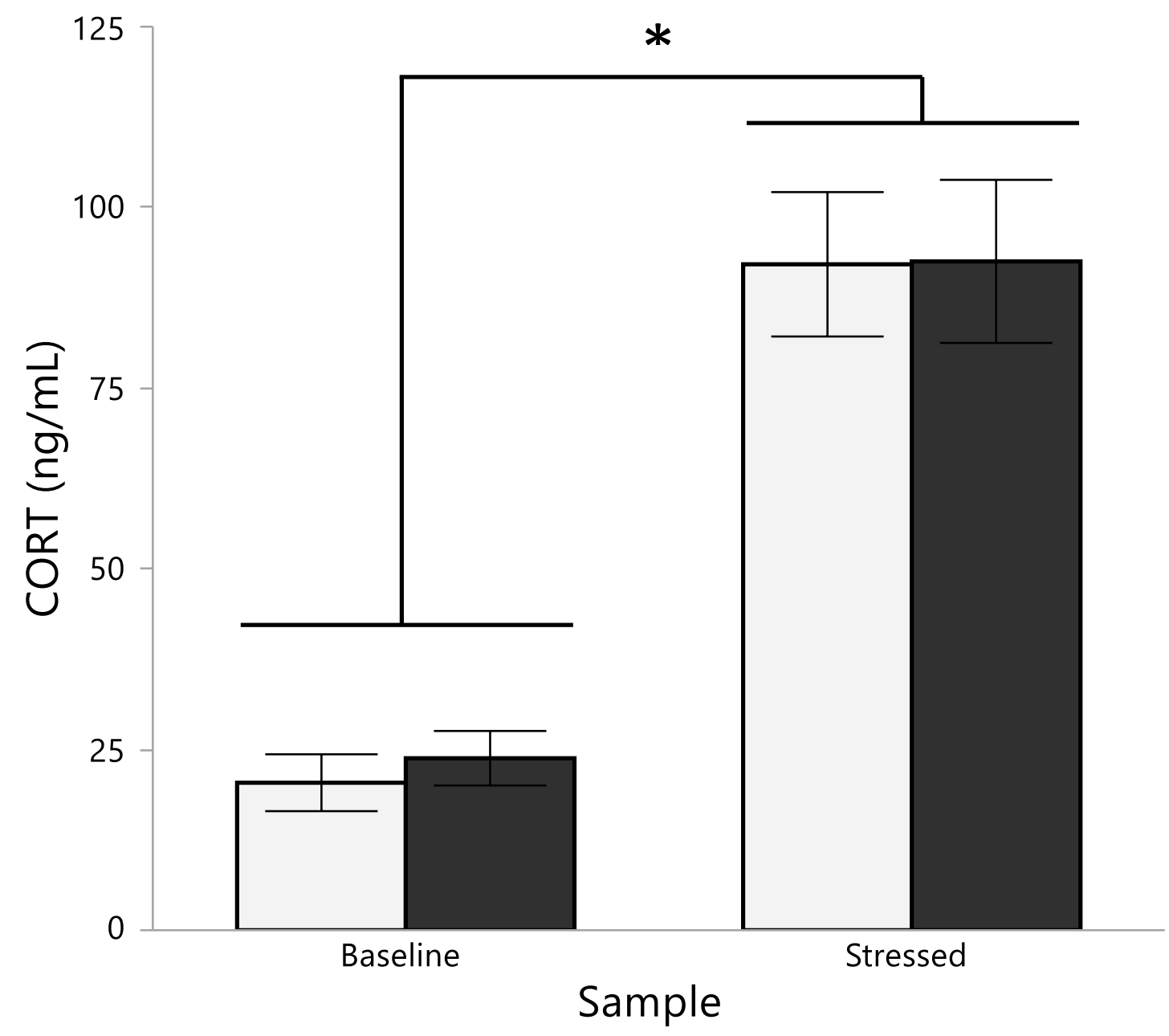

Figure 18. Stress reactivity of Crotalus oreganus after one hour of confinement in CORTimplanted (dark bars) and control snakes (light bars) at sampling period 3. Both groups showed a similar stress response, with no effect of treatment. Groups marked with an asterisk are significantly different. Error bars are \pm 1 SEM. 


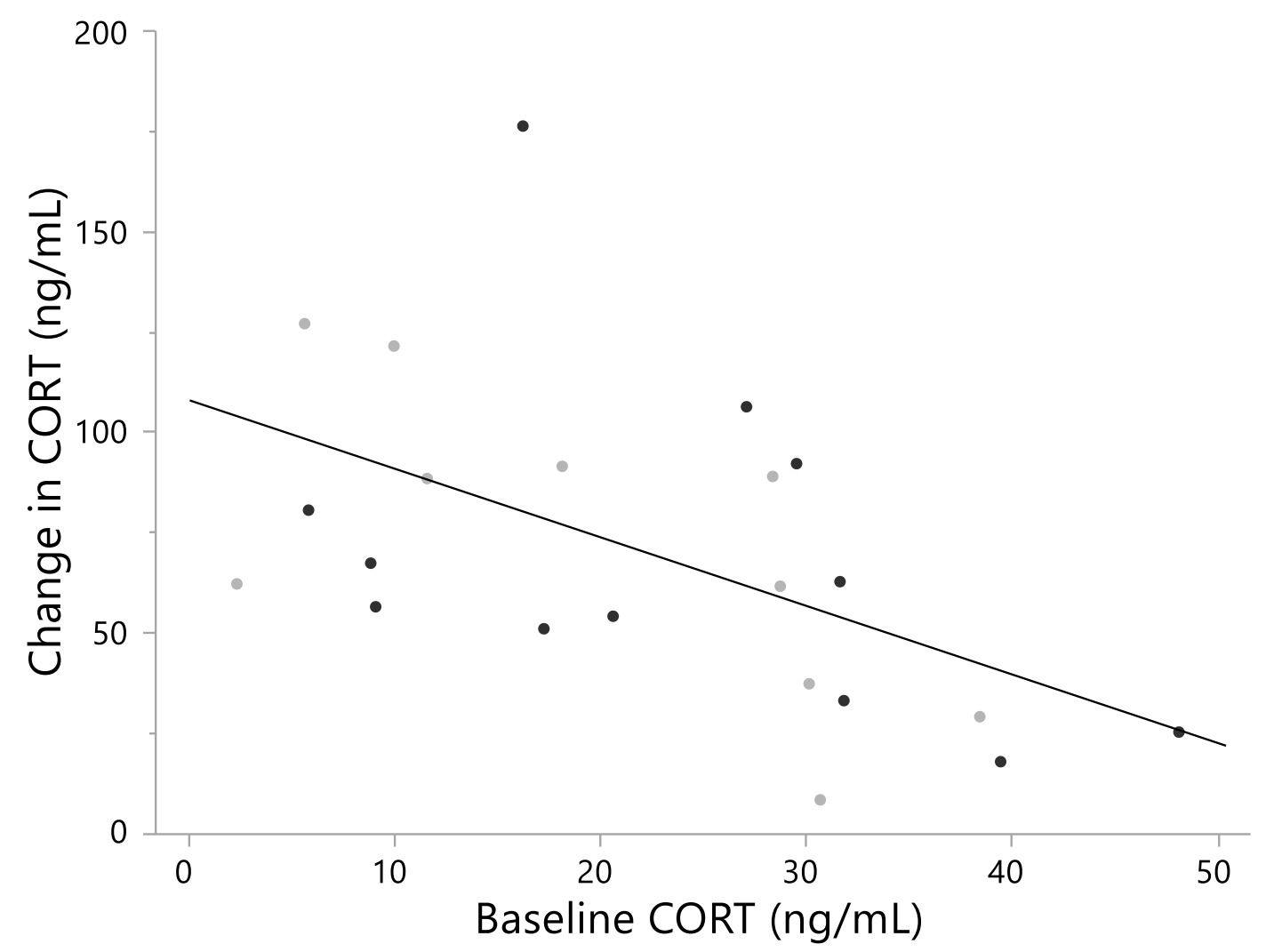

Figure 19. Magnitude of stress response in both control (light) and treatment (dark) Crotalus helleri after one hour of acute confinement stress. There was a trend for snakes with lower baseline CORT to exhibit a greater magnitude of CORT secretion in response to confinement stress than snakes with higher baseline CORT. 


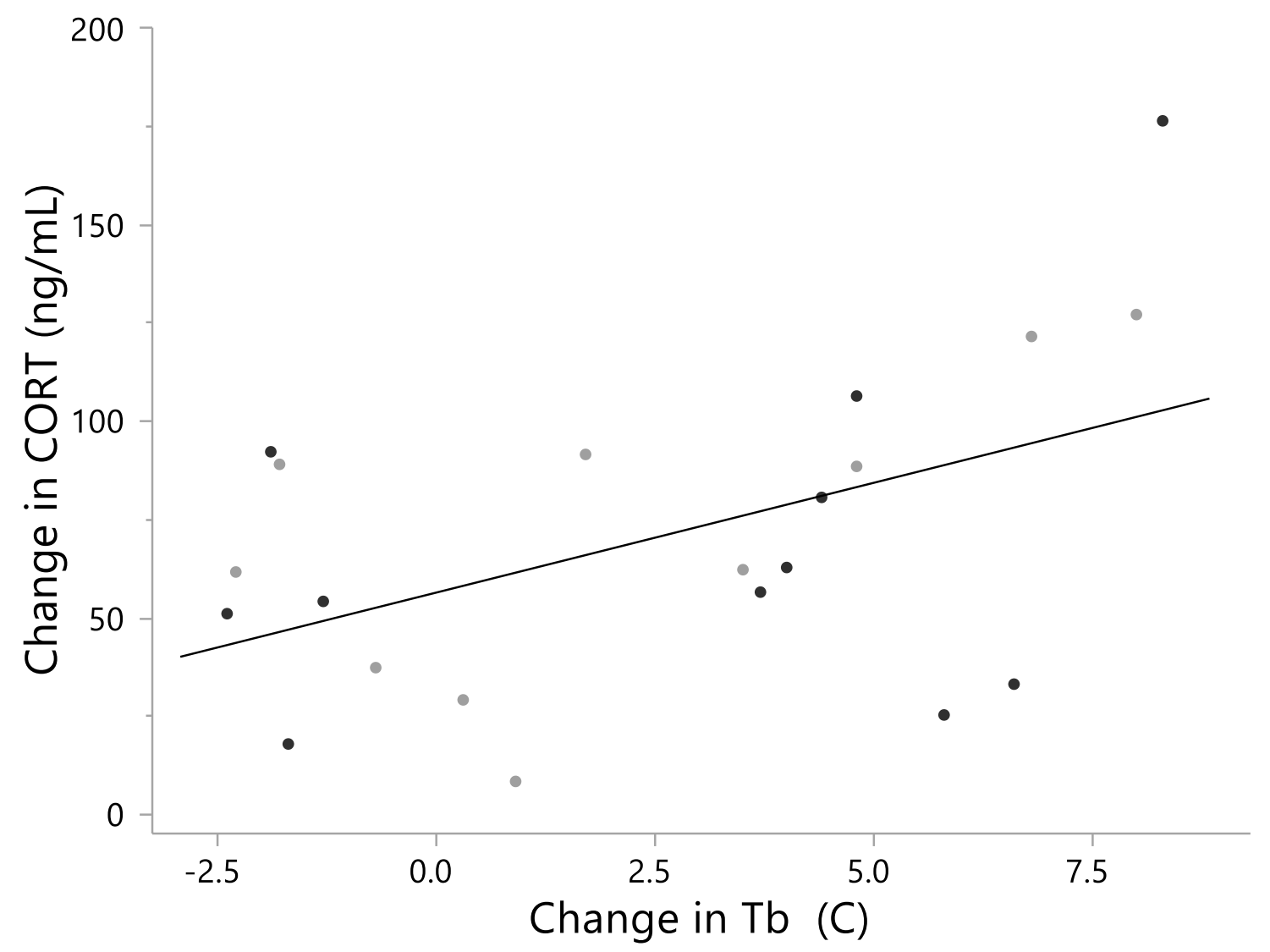

Figure 20. Relationship between change in $\mathrm{T}_{\mathrm{b}}$ and change in CORT in control (light) and treatment (dark) Crotalus helleri after one hour of acute confinement stress. There was a trend for snakes that increased in body temperature during confinement stress to exhibit a greater magnitude of CORT secretion. 


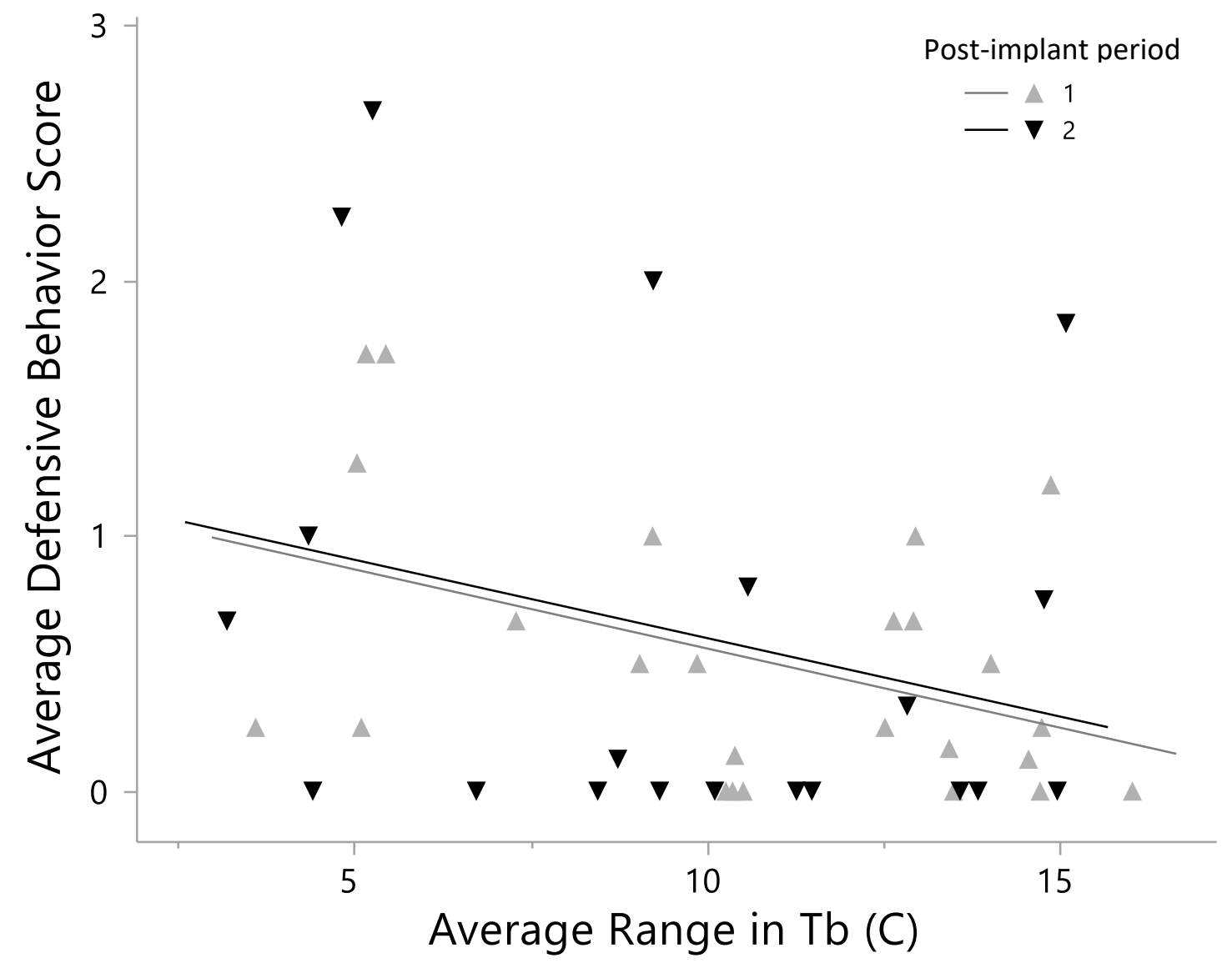

Figure 21. Average defensive behavior score during each tracking period for treatment (dark) and control (light) Crotalus helleri. Snakes with smaller ranges in $\mathrm{T}_{\mathrm{b}}$ were likely to have higher defensive behavior scores. Although all two datapoints from each snake are shown, each withinsnake sample was not considered independent. The RM ANCOVA accounted for repetition within individuals, so there was no pseudoreplication in our dataset. 


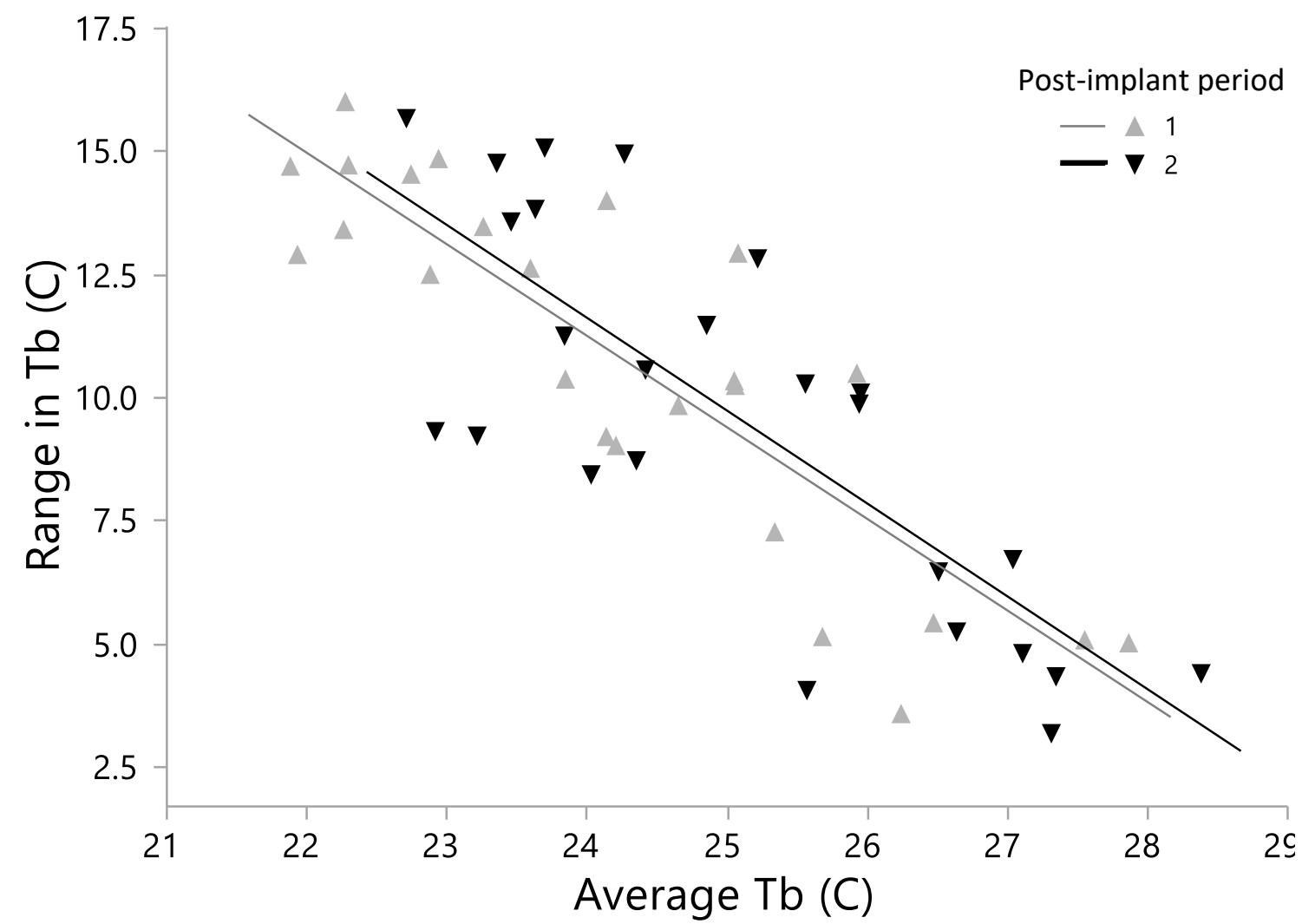

Figure 22. Average daily range in $\mathrm{T}_{\mathrm{b}}$ plotted against average $\mathrm{T}_{\mathrm{b}}$ for Crotalus helleri for each period. Range in $T_{b}$ strongly correlated to average $T_{b}$, with decreased range in $T_{b}$ associated with higher average $T_{b}$. Although two datapoints from each snake are shown, each within-snake sample was not considered independent. The RM ANCOVA accounted for repetition within individuals, so there was no pseudoreplication in our dataset. 


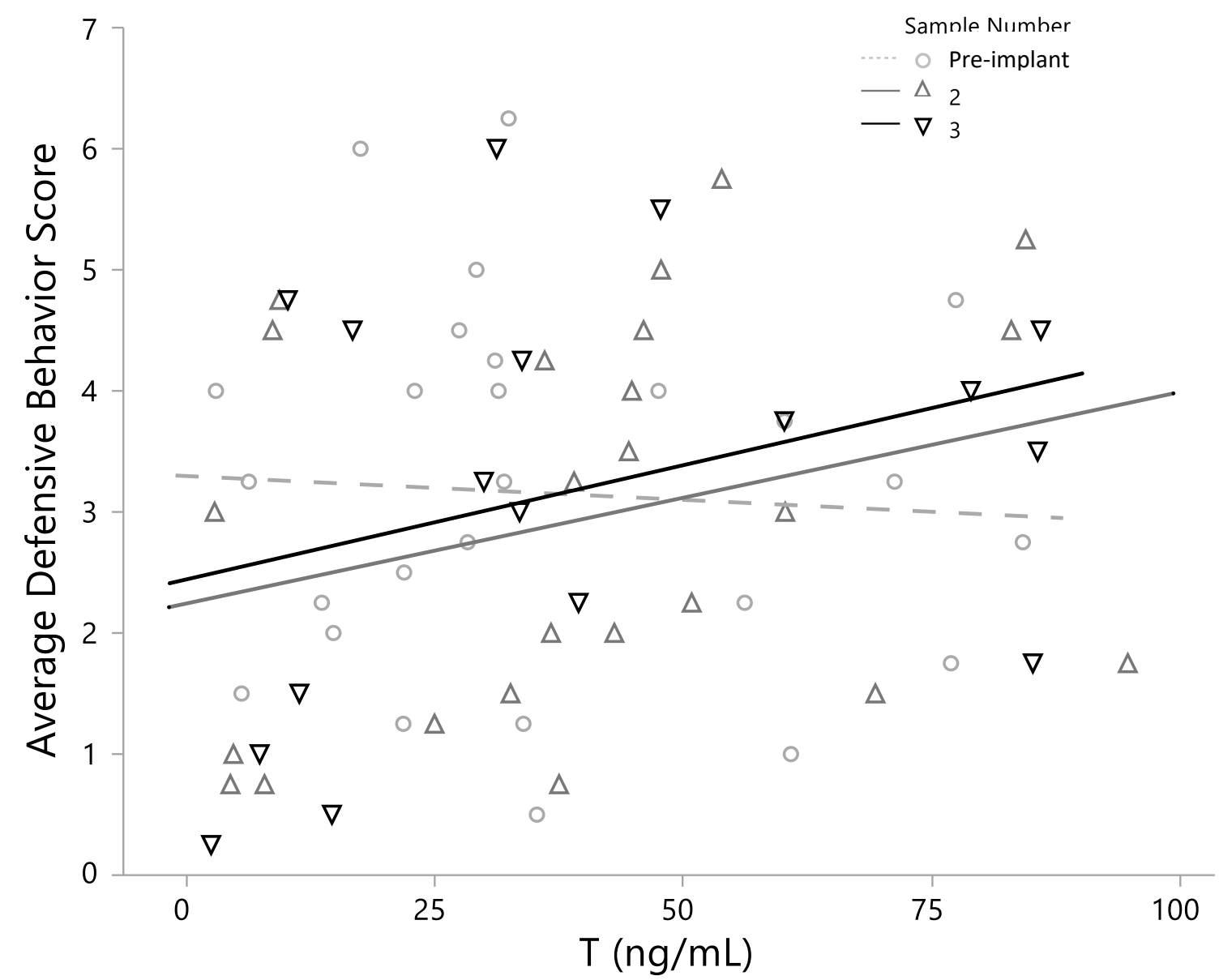

Figure 23. Average defensive behavior score during sampling of Crotalus helleri across three sampling periods (coded sequentially from light to dark). Testosterone was a significant covariate in the RM ANCOVA, with increasing Testosterone correlated with an increased defensive behavior score. Although all three samples from each snake are shown, each within-snake sample was not considered independent. The RM ANCOVA accounted for repetition within individuals, so there was no pseudoreplication in our dataset. 

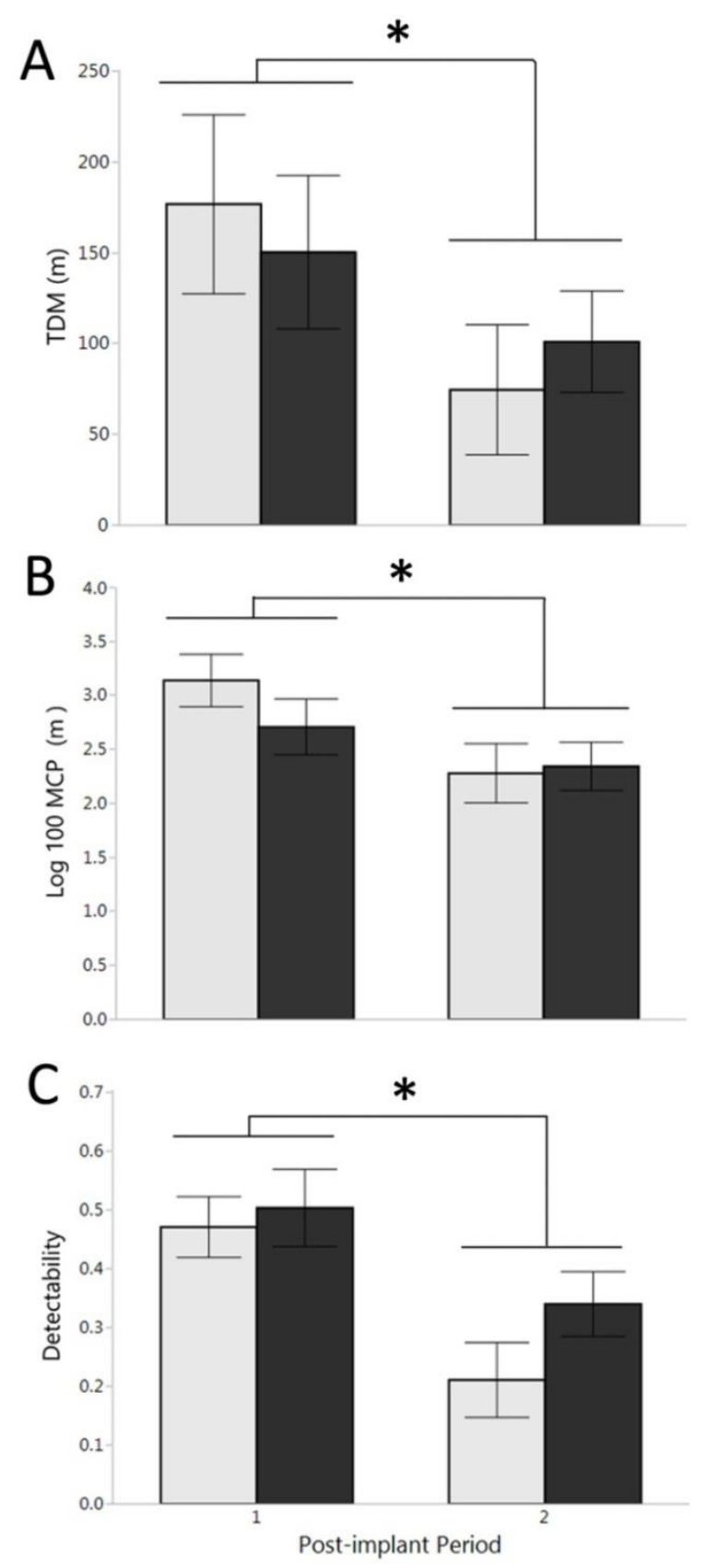

Figure 24. Spatial ecology parameters across each post-implant period in control (light bars) and treatment (dark bars) Crotalus helleri. Post-implant period 1: 0-15 days; Post-implant period 2: 15-20 days. Total distance moved (A), total area covered as calculated from Log $100 \mathrm{MCP}$ (B), and Detectability (C) were all unaffected by treatment. All snakes moved more (A), covered more area (B) and were more detectable in period 1 than period 2 (C). Groups marked with an asterisk are significantly different. Error bars are \pm 1 SEM. 


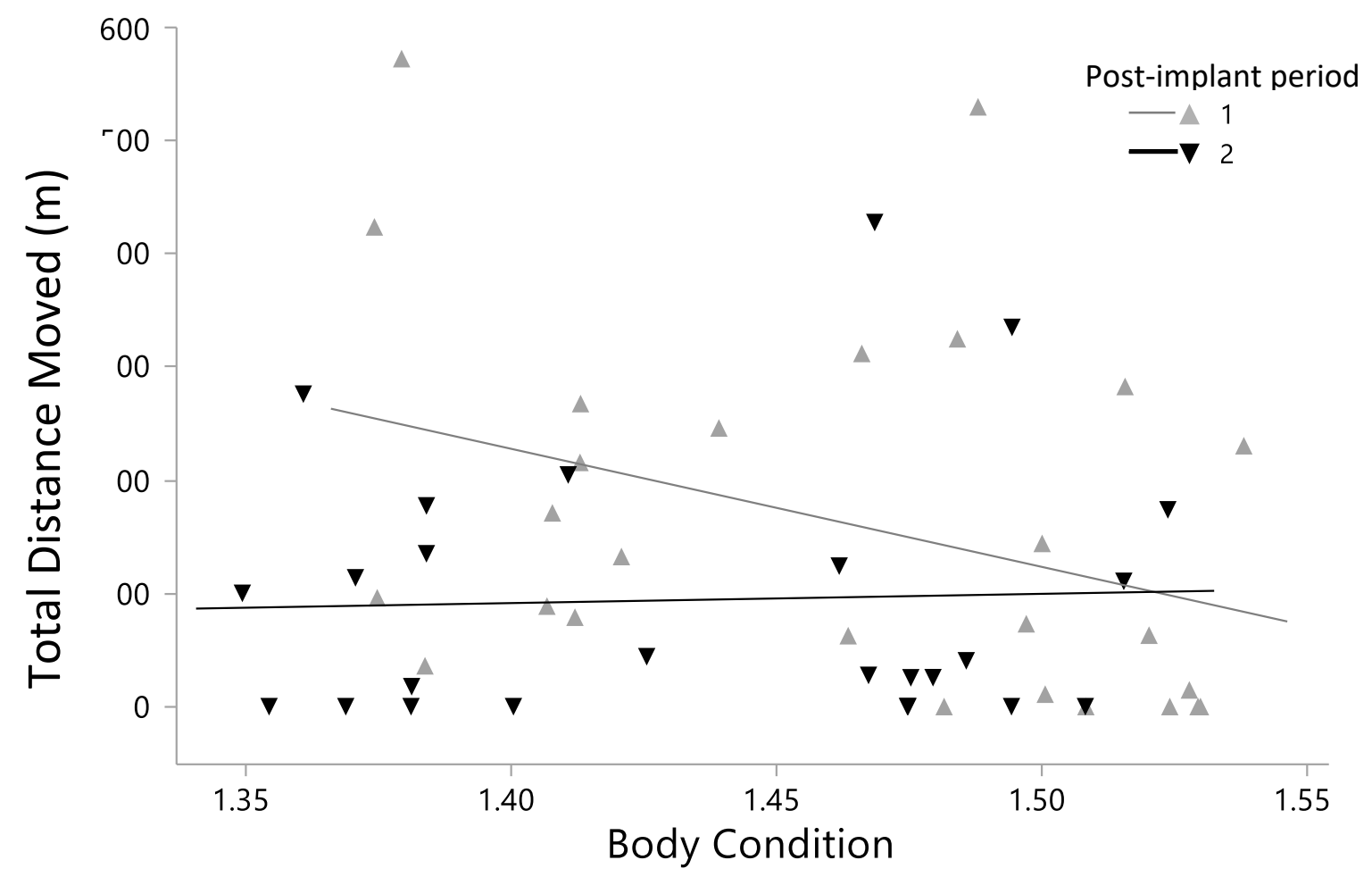

Figure 25. Total distance moved plotted against body condition of Crotalus helleri for each sampling period. There was a trend for an interaction between sampling period and body condition, where snakes were unaffected by body condition in period 2. Body condition was a significant covariate, with snakes in lower body condition moving more. Although two datapoints from each snake are shown, each within-snake sample was not considered independent. The RM ANCOVA accounted for repetition within individuals, so there was no pseudoreplication in our dataset. 
A

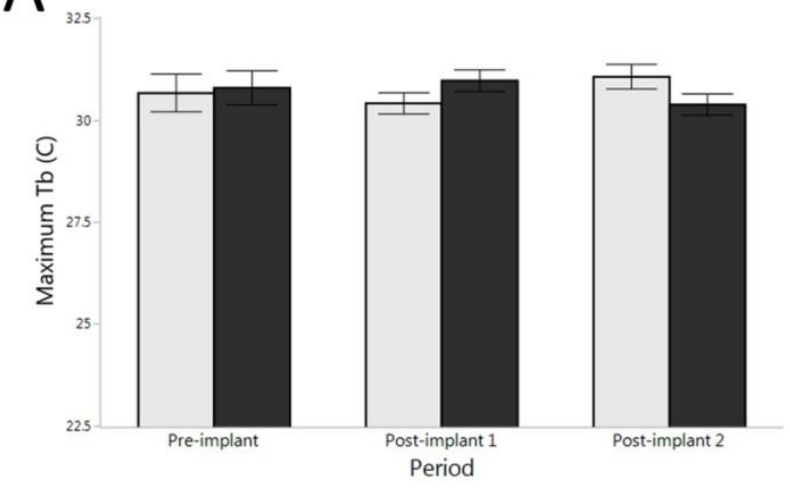

C

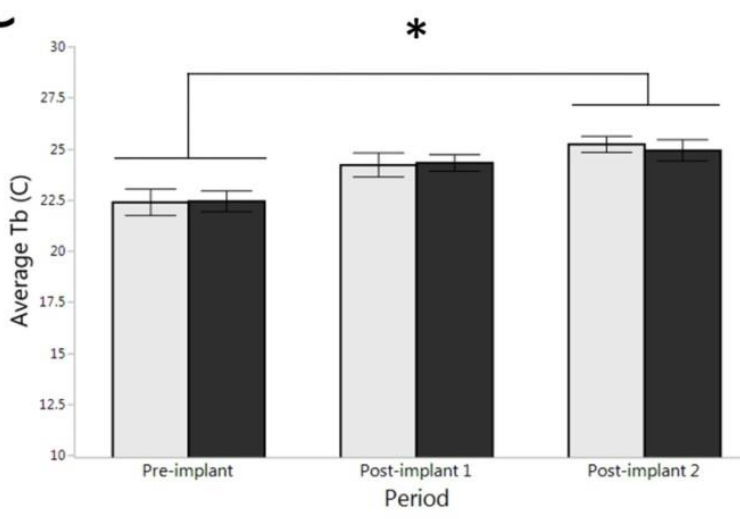

B

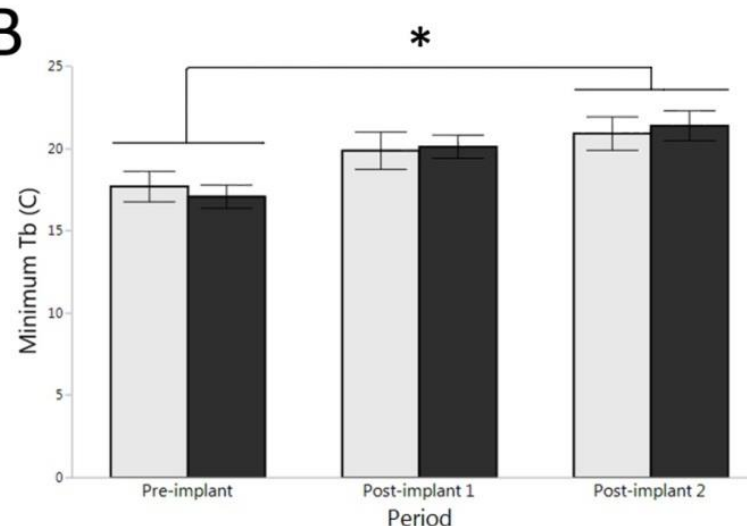

D

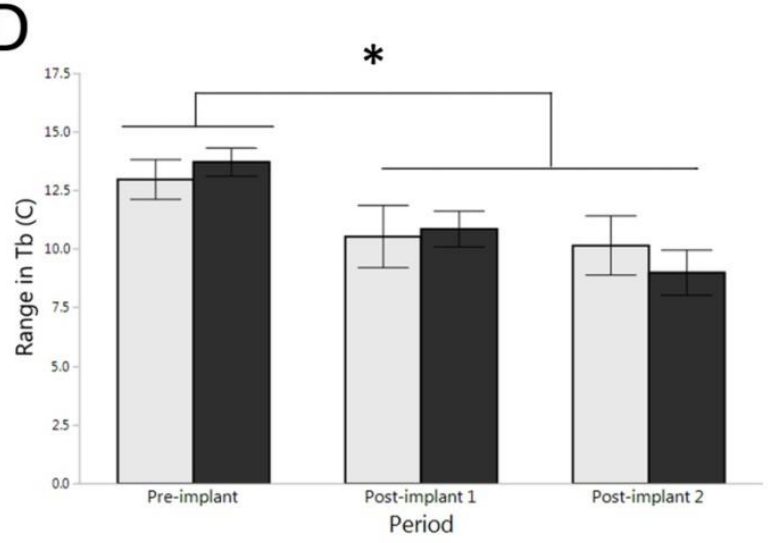

Figure 26. Average daily thermal parameters for Maximum $T_{b}(A)$. Minimum $T_{b}(B)$, Average $T_{b}(C)$, Range in $T_{b}(D)$ for treatment (dark bars) and control (light bars) Crotalus helleri at each sampling period. Maximum $\mathrm{T}_{\mathrm{b}}$ was unaffected by treatment or period. Minimum $\mathrm{T}_{\mathrm{b}}$ and Average $T_{b}$ were unaffected by treatment, but all snakes had lower minimum and average $T_{b}$ at pre-implant sampling compared to sample 2. Range in $T_{b}$ was unaffected by treatment, but all snakes had greater range in $T_{b}$ at pre-implant sampling compared to sample 1 and 2. Groups marked with an asterisk are significantly different. Error bars are \pm 1 SEM 


\section{Period}

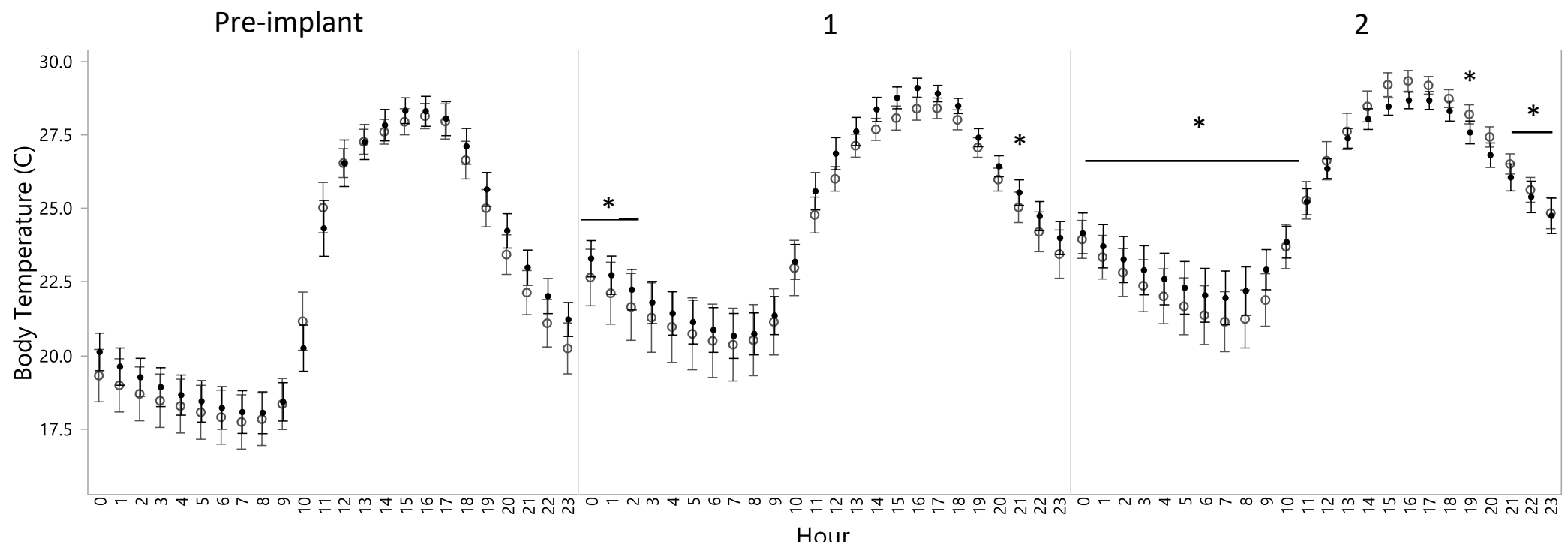

Figure 27. Average daily fluctuation in body temperature for control (open circles) and treatment (closed circles) Crotalus helleri at each sampling period. Maximum body temperatures reached were similar for each sampling period, while minimum temperatures and inflection points were higher in period 2 than pre-implant, and a few cool-down temperatures were different in period 1 than pre-implant.

Period 2 and 1 were not significantly different from one another. Asterisks indicate significant differences compared to the same hours during the Pre-implant period. Error bars are \pm 1 SEM. 


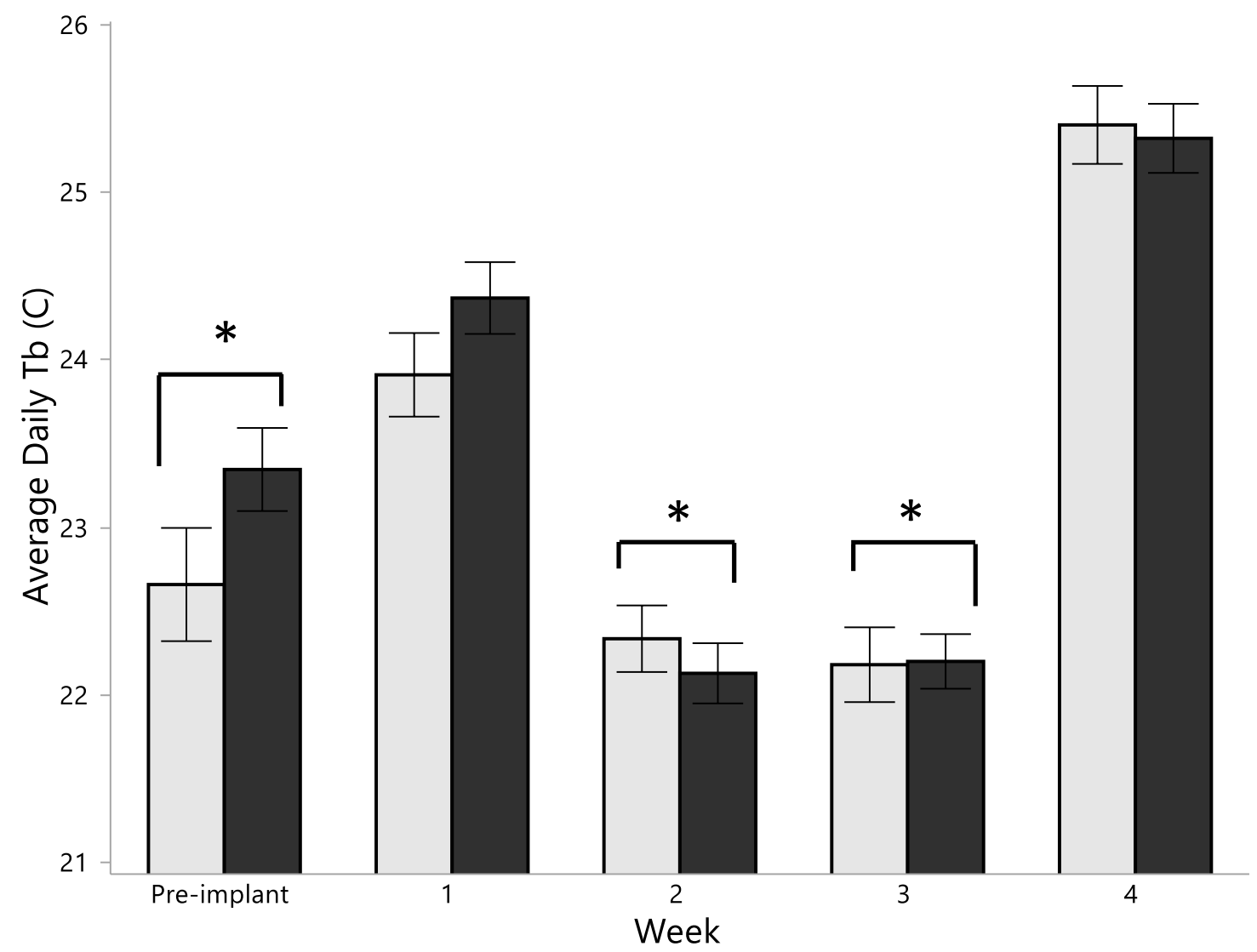

Figure 28. Average $\mathrm{T}_{\mathrm{b}}$ for treatment (dark bars) and control (light bars) Crotalus helleri for the week before implant and four weeks post-implant. Treatment snakes pre-implant had higher $\mathrm{T}_{\mathrm{b}}$, this effect was reversed in weeks 2 and 3 post-implant. Asterisks indicate significant differences between treatment groups. Error bars are \pm 1 SEM. 


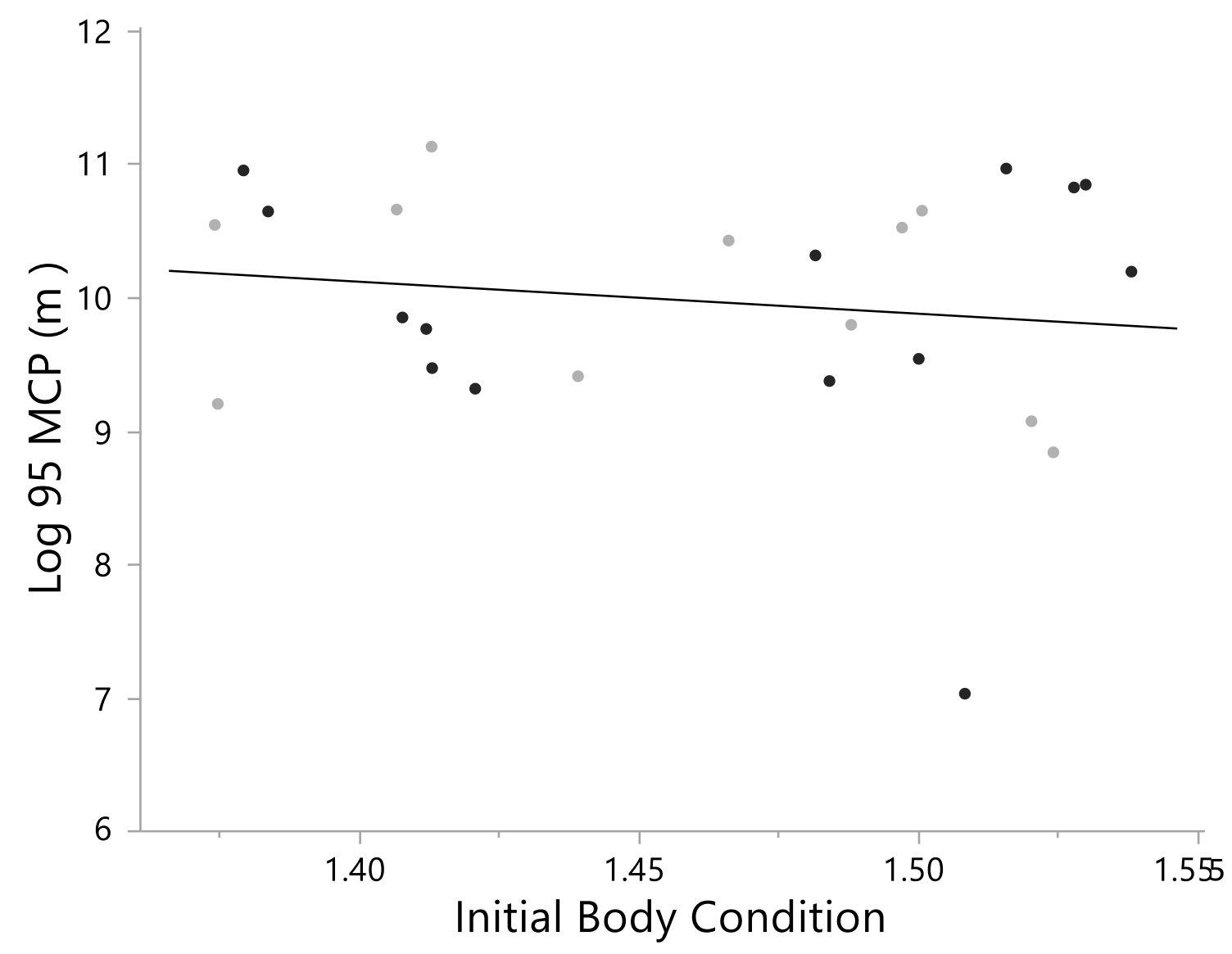

Figure 29. Home range size of control (light) and treatment (dark) Crotalus helleri as measured with 95\% MCPs. There was no effect of treatment on home range size, but initial body condition at implantation was a significant covariate in the RM ANCOVA, with snakes in poorer body condition having larger home ranges. 


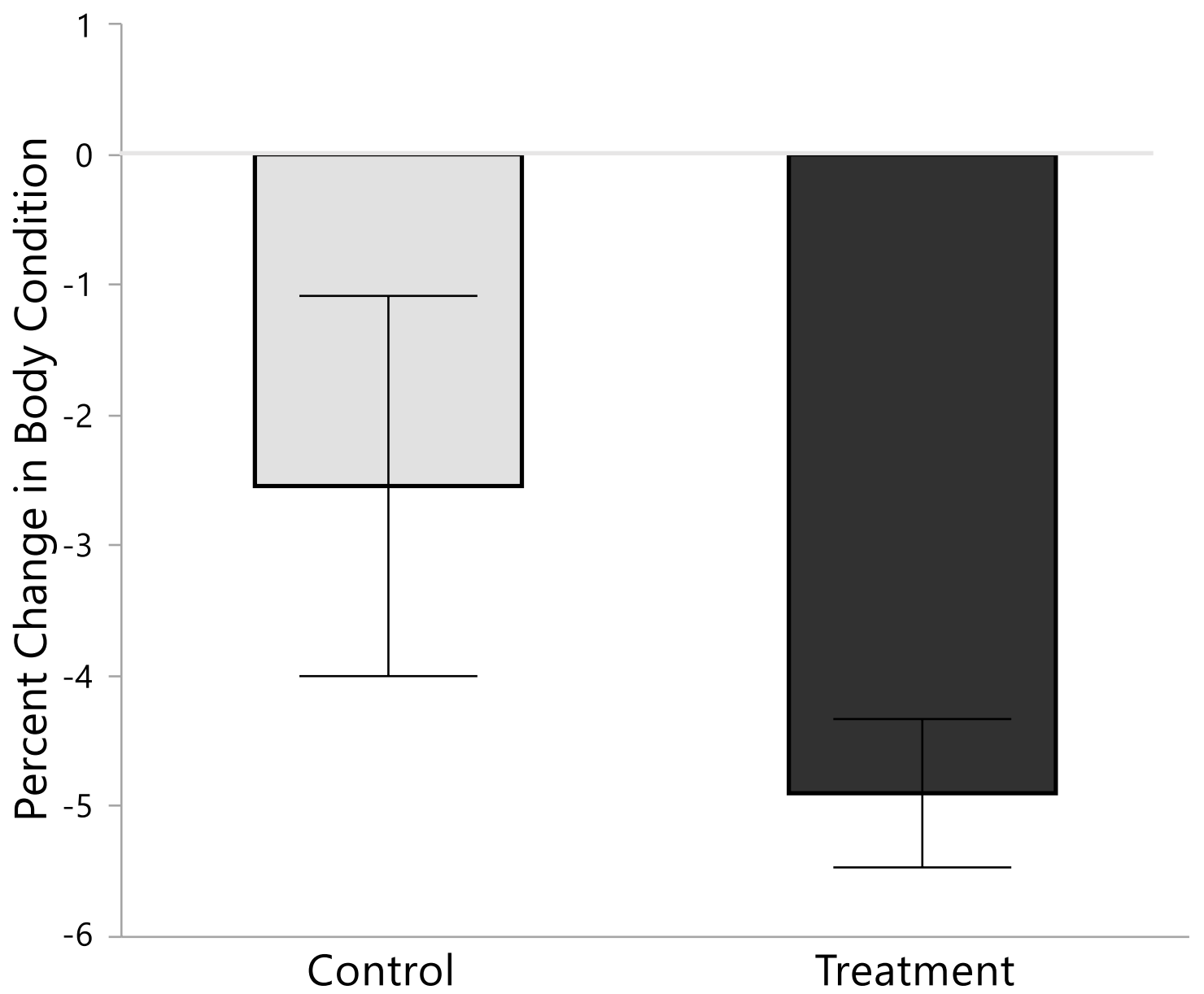

Figure 30. Percent change in body condition from implant date for Crotalus helleri. All snakes decreased in body condition over the study, but there was no difference in body condition based on treatment in the one-way ANOVA. Error bars are \pm 1 SEM. 


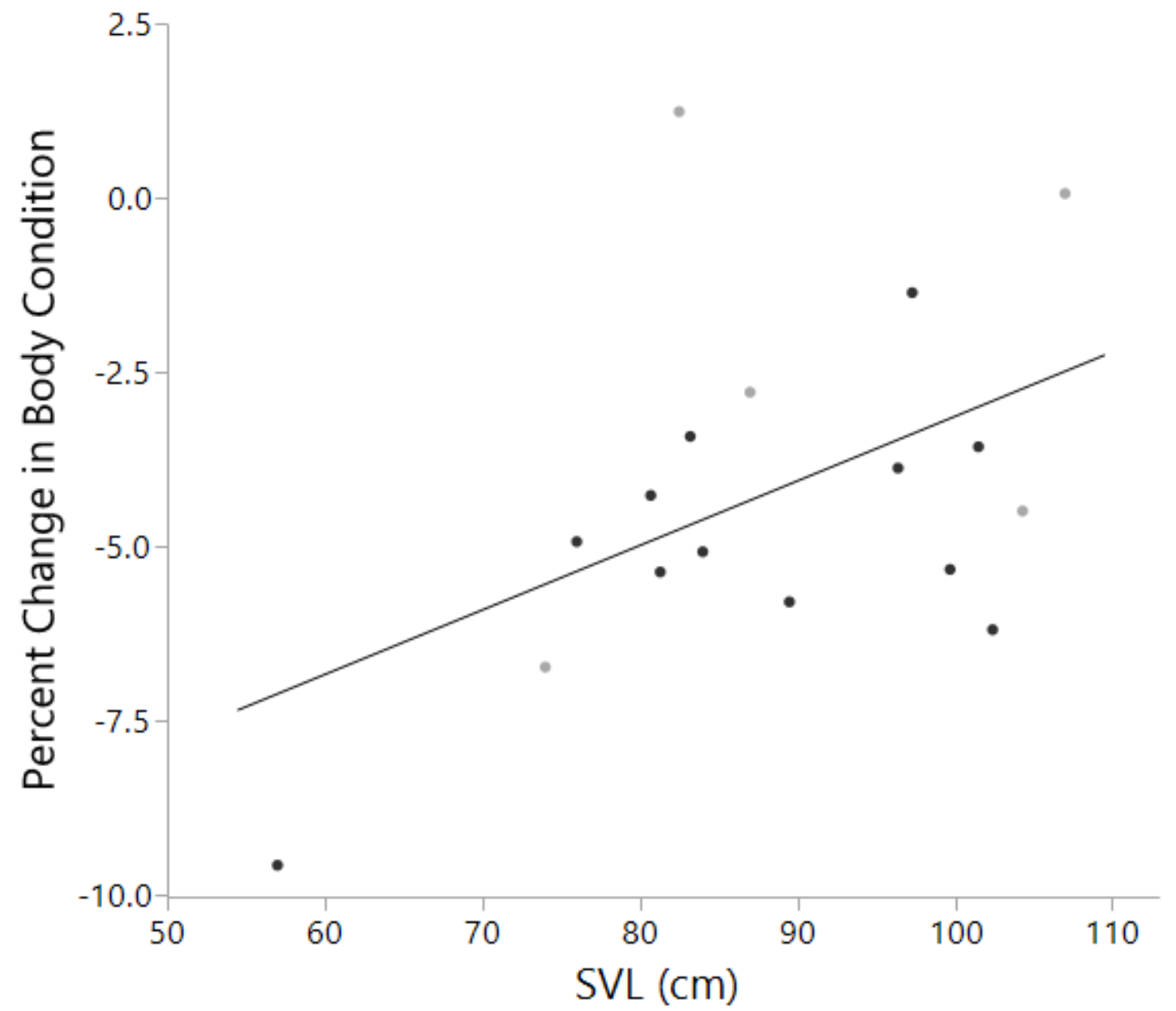

Figure 31. Percent change in body condition from implant date to end of study for control (light) and treatment (dark) Crotalus helleri. SVL was marginally significant, with a trends for smaller snakes to have greater negative change in body condition. 


\section{REFERENCES}

Aitchison, J. (1986). The statistical analysis of compositional data. Chapman\&Hall:

London, United Kingdom: Reprinted in 2003 by Blackburn Press.

Akani, G. C., Ebere, N., Franco, D., Eniang, E. a, Petrozzi, F., Politano, E., \& Luiselli, L. (2013). Correlation between annual activity patterns of venomous snakes and rural people in the Niger Delta, southern Nigeria. The Journal of Venomous Animals and Toxins Including Tropical Diseases, 19(1), 2. http://doi.org/10.1186/1678-9199-19-2

Alape-Girón, A., Sanz, L., Flores-Díaz, M., Madrigal, M., Sasa, M., \& Calvete, J. J. (2008). Snake venomics of the lancehead pitviper Bothrops asper: Geographic, individual, and ontogenetic variations research articles. Journal of Proteome Research, 7, 3556-3571.

Arnold, B. Y. S. J., \& Bennett, A. F. (1984). Antipredator Displays in the Garter Snake, $1108-1118$.

Baird, T. A., Lovern, M. B., \& Shine, R. (2014). Heightened aggression and winning contests increase corticosterone but decrease testosterone in male Australian water dragons. Hormones and Behavior, 66(2), 393-400.

http://doi.org/10.1016/j.yhbeh.2014.05.008

Baker, M. R., Gobush, K. S., \& Vynne, C. H. (2013). Review of factors influencing stress hormones in fish and wildlife. Journal for Nature Conservation, 21(5), 309-318. http://doi.org/10.1016/j.jnc.2013.03.003 
Bauder, J. M., Macey, J. N., Stohlgren, K. M., Day, A., Snow, F., Safer, A., ...

Stevenson, D. J. (2015). Factors influencing the display of multiple defensive behaviors in Eastern indigo snakes (Drymarchon couperi). Herpetological Conservation and Biology, 10(2), 559-571.

Baugh, A. T., Oers, K. van, Dingemanse, N. J., \& Hau, M. (2014). Baseline and stressinduced glucocorticoid concentrations are not repeatable but covary within individual great tits (Parus major). General and Comparative Endocrinology, 208, 154-163. http://doi.org/10.1016/j.ygcen.2014.08.014

Belliure, J., \& Clobert, J. (2004). Behavioral sensitivity to corticosterone in juveniles of the wall lizard, Podarcis muralis. Physiology and Behavior, 81(1), 121-127. http://doi.org/10.1016/j.physbeh.2004.01.008

Berger, S., Martin, L. B., Wikelski, M., Romero, L. M., Kalko, E. K. V, Vitousek, M. N., \& Rödl, T. (2005). Corticosterone suppresses immune activity in territorial Galápagos marine iguanas during reproduction. Hormones and Behavior, 47(4), 419-429. http://doi.org/10.1016/j.yhbeh.2004.11.011

Biardi, J. E., Nguyen, K. T., Lander, S., Whitley, M., \& Nambiar, K. P. (2011). A rapid and sensitive fluorometric method for the quantitative analysis of snake venom metalloproteases and their inhibitors. Toxicon, 57(2), 342-347. http://doi.org/10.1016/j.toxicon.2010.12.014

Bradshaw, D. (2007). Environmental endocrinology. General and Comparative Endocrinology, 152(2-3), 125-141. http://doi.org/10.1016/j.ygcen.2006.12.026

Breuner, C. W., Greenberg, a L., \& Wingfield, J. C. (1998). Noninvasive corticosterone 
treatment rapidly increases activity in Gambel's white-crowned sparrows (Zonotrichia leucophrys gambelii). General and Comparative Endocrinology, 111, 386-394. http://doi.org/10.1006/gcen.1998.7128

Breuner, C. W., Delehanty, B., \& Boonstra, R. (2013). Evaluating stress in natural populations of vertebrates: Total CORT is not good enough. Functional Ecology, 27(1), 24-36. http://doi.org/10.1111/1365-2435.12016

Busch, D. S., \& Hayward, L. S. (2009). Stress in a conservation context: A discussion of glucocorticoid actions and how levels change with conservation-relevant variables. Biological Conservation, 142(12), 2844-2853. http://doi.org/10.1016/j.biocon.2009.08.013

Bush, S. P., Green, S. M., Moynihan, J. A., Hayes, W. K., \& Cardwell, M. D. (2002). Crotalidae polyvalent immune fab (ovine) antivenom is efficacious for envenomations by Southern Pacific rattlesnakes (Crotalus helleri). Annals of Emergency Medicine, 409(6), 619-624. http://doi.org/10.1067/mem.2002.129939

Calvete, J., Sanz, L., Angulo, Y., Lomonte, B., Gutiérrez, J.M., (2009). Venoms, venomics, antivenomics. FEBS Letters, 583, 1736-1743.

Calvete, J., Sanz, L., Pérez, A., Borges, A., Vargas, A.M., Lomonte, B., Angulo, Y., Gutiérrez,J., Chalkidis, H., Mourão, R., Furtado, M., Moura-Da-Silva, A., (2011). Snake population venomics and antivenomics of Bothrops atrox: Paedomorphism along its transamazonian dispersal and implications of geographic venom variability on snakebite management. Journal of Proteomics, 74, 510-527.

Casewell, N. R., Wagstaff, S. C., Wüster, W., Cook, D. A. N., Bolton, F. M. S., King, S. 
I., ... Harrison, R. A. (2014). Medically important differences in snake venom composition are dictated by distinct postgenomic mechanisms. PNAS, 111(25), 9205-9210. http://doi.org/10.1073/pnas.1405484111

Cash, W B, Holberton, R. L. (1999). Effects of exogenous corticosterone on locomotor activity in the red-eared slider turtle,Trachemys scripta elegans. Journal of Experimental Zoology, 644(July 1998), 284:637-644. Retrieved from https://www.researchgate.net/publication/12768178_Effects_of_exogenous_corticos terone_on_locomotor_activity_in_the_redeared_slider_turtleTrachemys_scripta_elegans

Chippaux, J.-P., Williams, V., \& White, J. (1991). Snake venom variability: methods of study, results and interpretation. Toxicon, 29(11), 1279-1303. http://doi.org/10.1016/j.limno.2013.04.005

Chouhan and Sharma, (2009). Ecology of tropical deserts with special reference to biogeography and evolution of desert animals. In: Tropical Biology and Conservation Management, Volume IX. Chouhan and Sharma (Eds). Eolss Publishers Co., Oxford, United Kingdom.

Chuang, T.-D., Pearce, W. J., \& Khorram, O. (2015). miR-29c induction contributes to downregulation of vascular extracellular matrix proteins by glucocorticoids. American Journal of Physiology. Cell Physiology, 309(2), C117-25. http://doi.org/10.1152/ajpcell.00254.2014

Cockrem, J. F. (2013). Individual variation in glucocorticoid stress responses in animals. General and Comparative Endocrinology, 181, 45-58. 
http://doi.org/10.1016/j.ygcen.2012.11.025

Cooper, W., Caldwell, J., \& Vitt, L. (2008). Effective crypsis and its maintenance by immobility in Craugastor frogs. Copeia, 2008(3), 527-532. http://doi.org/10.1643/CE-O7-056

Cree, A., Amey, A. P., \& Whittier, J. M. (2000). Lack of consistent hormonal responses to capture during the breeding season of the bearded dragon, Pogona barbata. Comparative Biochemistry and Physiology - A Molecular and Integrative Physiology, 126(2), 275-285. http://doi.org/10.1016/S1095-6433(00)00210-5

Cree, A., Tyrrell, C. L., Preest, M. R., Thorburn, D., \& Guillette, L. J. (2003). Protecting embryos from stress: Corticosterone effects and the corticosterone response to capture and confinement during pregnancy in a live-bearing lizard (Hoplodactylus maculatus). General and Comparative Endocrinology, 134(3), 316-329. http://doi.org/10.1016/S0016-6480(03)00282-X

Currier, R. (2012). Investigating venom synthesis: exploring the composition, variation and gene expression dynamics of Bitis arietans venom, (August). Retrieved from http://research-archive.liv.ac.uk/8153/

Currier, R. B., Calvete, J. J., Sanz, L., Harrison, R. A., Rowley, P. D., \& Wagstaff, S. C. (2012). Unusual stability of messenger RNA in snake venom reveals gene expression dynamics of venom replenishment. PloS One, 7(8), e41888. http://doi.org/10.1371/journal.pone.0041888

Currier, R. B., Harrison, R. A., Rowley, P. D., Laing, G. D., \& Wagstaff, S. C. (2010). Intra-specific variation in venom of the African puff adder (Bitis arietans): 
Differential expression and activity of snake venom metalloproteinases (SVMPs). Toxicon, 55(4), 864-873. http://doi.org/10.1016/j.toxicon.2009.12.009

Dayger, C. A., Cease, A. J., \& Lutterschmidt, D. I. (2013). Hormones and Behavior Responses to capture stress and exogenous corticosterone vary with body condition in female red-sided garter snakes ( Thamnophis sirtalis parietalis ). Hormones and Behavior, 64, 748-754. http://doi.org/10.1016/j.yhbeh.2013.09.003

Dean, R. B., \& Dixon W. J. (1951). Simplified Statistics for Small Numbers of Observations. Analytical Chemistry, 23(4), 636-638.

DeNardo, D. F., \& Licht, P. (1993). Effects of corticosterone on social behavior of male lizards. Hormones and Behavior, 27, 184-199.

DeNardo, D. F., \& Sinervo, B. (1994). Effects of corticosterone on activity and home range size of free-ranging male lizards. Hormones and Behavior, 28, 53-65.

DesRochers, D. W., Reed, J. M., Awerman, J., Kluge, J. A., Wilkinson, J., van Griethuijsen, L. I., ... Romero, L. M. (2009). Exogenous and endogenous corticosterone alter feather quality. Comparative Biochemistry and Physiology Part A: Molecular \& Integrative Physiology, 152(1), 46-52. http://doi.org/10.1016/j.cbpa.2008.08.034

Dhabhar, F. S. (2009). Enhancing versus suppressive effects of stress on immune function: Implications for immunoprotection and immunopathology. NeuroImmunoModulation, 16(5), 300-317. http://doi.org/10.1159/000216188

Dickens, M. J., \& Romero, L. M. (2013). A consensus endocrine profile for chronically 
stressed wild animals does not exist. General and Comparative Endocrinology, 191, 177-189. http://doi.org/10.1016/j.ygcen.2013.06.014

Duan, Y., Fu, W., Wang, S., Ni, Y., \& Zhao, R. (2014). Effects of tonic immobility ( TI ) and corticosterone ( CORT ) on energy status and protein metabolism in pectoralis major muscle of broiler chickens. Comparative Biochemistry and Physiology, Part A, 169, 90-95. http://doi.org/10.1016/j.cbpa.2013.12.019

Dunlap, K. D., \& Wingfield, J. C. (1995). External and internal influences on indices of physiological stress. I. Seasonal and population variation in adrenocortical secretion of free-living lizards, Sceloporus occidentalis. The Journal of Experimental Zoology, 27l(1), 36-46. http://doi.org/10.1002/jez.1402710105

Dupoué, A., Brischoux, F., Lourdais, O., \& Angelier, F. (2013). Influence of temperature on the corticosterone stress-response: An experiment in the Children's python (Antaresia childreni). General and Comparative Endocrinology, 193, 178-184. http://doi.org/10.1016/j.ygcen.2013.08.004

Duvall, D., \& Schuett, G. W. (1997). Straight-line movement and competitive mate searching in prairie rattlesnakes, Crotalus viridis viridis. Animal Behaviour, 54(2), 329-334. http://doi.org/10.1006/anbe.1996.0418

Escribano-Avila, A., Pettorelli, N., Virgós, E., Lara-Romero, C., Lozano, J., Barja, I., ... Puerta, M. (2013). Testing Cort-Fitness and Cort-Adaptation hypotheses in a habitat suitability gradient for roe deer. Acta Oecologica, 53, 38-48. http://doi.org/10.1016/j.actao.2013.08.003

Fauvel, T., Brischoux, F., Briand, M. J., \& Bonnet, X. (2012). Do researchers impact 
their study populations? Assessing the effect of field procedures in a long term population monitoring of sea kraits. Amphibia-Reptilia, 33(3-4), 365-372. http://doi.org/10.1163/15685381-00002839

Filzmoser, P., Hron, K., Reimann, C. (2009) Principal component analysis for compositional data with outliers. Environmetrics, 20, 621-632.

Fitch, H. S. (1965). An ecological study of the garter snake, Thamnophis sirtalis. University of Kansas Public Museum of Natural History, 15, 493-564.

Freitas-de-Sousa, L., Amazonas, D., Sousa, L., Sant'Anna, S., Nishiyama Jr, M., Serrano, S., Junqueira-de-Azevedo, I., Chalkidis, H., Moura-da-Silva, A., Mourão, R., 2015. Comparison of venoms from wild and long-term captive Bothrops atrox snakes and characterization of Batroxrhagin, the predominant class PIII metalloproteinase from the venom of this species. Biochimie, 118, 60-70.

French, W. J., Hayes, W. K., Bush, S. P., Cardwell, M. D., Bader, J. O., \& Rael, E. D. (2004). Mojave toxin in venom of Crotalus helleri (Southern Pacific Rattlesnake): molecular and geographic characterization. Toxicon, 44(7), 781-791. http://doi.org/10.1016/j.toxicon.2004.08.008

Fry, B. (2005). From genome to "venome": molecular origin and evolution of the snake venom proteome inferred from phylogenetic analysis of toxin sequences and related body proteins. Genome Research, 15(3), 403-420. http://doi.org/10.1101/gr.3228405

Fry, B. G., \& Wüster, W. (2004). Assembling an arsenal: origin and evolution of the snake venom proteome inferred from phylogenetic analysis of toxin sequences. 
Molecular Biology and Evolution, 21(5), 870-883.

http://doi.org/10.1093/molbev/msh091

Gaeggeler, H.-P. (2005). Mineralocorticoid versus glucocorticoid receptor occupancy

mediating aldosterone-stimulated sodium transport in a novel renal cell line. Journal of the American Society of Nephrology, 16(4), 878-891.

http://doi.org/10.1681/ASN.2004121110

Gibbons, J. W., \& Dorcas, M. E. (2002). Defensive Behavior of Cottonmouths

(Agkistrodon piscivorus) toward Humans. Copeia, 2002(1), 195-198. http://doi.org/10.1643/0045-8511(2002)002[0195:DBOCAP]2.0.CO;2

Gibbs, H. L., Sanz, L., \& Calvete, J. J. (2009). Snake population venomics: proteomicsbased analyses of individual variation reveals significant gene regulation effects on venom protein expression in Sistrurus rattlesnakes. Journal of Molecular Evolution, 68(2), 113-25. http://doi.org/10.1007/s00239-008-9186-1

Gibbs, H. L., Sanz, L., Chiucchi, J. E., Farrell, T. M., \& Calvete, J. J. (2011). Proteomic analysis of ontogenetic and diet-related changes in venom composition of juvenile and adult Dusky Pigmy rattlesnakes (Sistrurus miliarius barbouri). Journal of Proteomics, 74(10), 2169-2179. http://doi.org/10.1016/j.jprot.2011.06.013

Glanemann, C., Loos, a, Gorret, N., Willis, L. B., O’Brien, X. M., Lessard, P. a, \& Sinskey, a J. (2003). Disparity between changes in mRNA abundance and enzyme activity in Corynebacterium glutamicum: implications for DNA microarray analysis. Applied Microbiology and Biotechnology, 61(1), 61-68.

http://doi.org/10.1007/s00253-002-1191-5 
Glaudas, X., Farrell, T. M., \& May, P. G. (2005). Defensive behavior of free-ranging pygmy rattlesnakes (Sistrurus miliarius). Copeia, 2005(1), 196-200. http://doi.org/10.1643/CH-03-253R1

Goessling, J. M., Kennedy, H., Mendonca, M. T., \& Wilson, A. E. (2015). A metaanalysis of plasma corticosterone and heterophil: Lymphocyte ratios - is there conservation of physiological stress responses over time? Functional Ecology, 29, 1189-1196. http://doi.org/10.1111/1365-2435.12442

Golinski, A., Kubicka, L., John-Alder, H., \& Kratochvíl, L. (2014). Elevated testosterone is required for male copulatory behavior and aggression in Madagascar ground gecko (Paroedura picta). General and Comparative Endocrinology, 205, 133-141. http://doi.org/10.1016/j.ygcen.2014.05.012

Goode, M. J., \& Duvall, D. (1988). Body Temperature and Defensive Behaviour of Freeranging Prairie Rattlesnakes, Crotalus viridis viridis. Animal Behaviour, 38(2), 360362.

Gregory-Dwyer, V., Egen, N., Bianchi Bosisio, A., Righetti, P., \& Russell, F. (1986). An isoelectric focusing study of seasonal variation in rattlesnake venom proteins. Toxicon, 24(10), 995-1000.

Gubensek, F., Sket, D., Turk, V., Lebez, D., 1974. Fractionation of Vipera ammodytes venom and seasonal variation of its composition. Toxicon 12, 167-171.

Guércio, R. A. P., Shevchenko, A., Shevchenko, A., López-Lozano, J. L., Paba, J., Sousa, M. V, \& Ricart, C. A. O. (2006). Ontogenetic variations in the venom proteome of the Amazonian snake Bothrops atrox. Proteome Science, 4(1), 11. 
http://doi.org/10.1186/1477-5956-4-11

Hamann, M., Jessop, T. S., \& Schäuble, C. S. (2007). Fuel use and corticosterone dynamics in hatchling green sea turtles (Chelonia mydas) during natal dispersal. Journal of Experimental Marine Biology and Ecology, 353(1), 13-21. http://doi.org/10.1016/j.jembe.2007.08.017

Harms, C., Albrecht, K., Harms, U., Seidel, K., Hauck, L., Baldinger, T., ... Hörtnagl, H. (2007). Phosphatidylinositol 3-Akt-kinase-dependent phosphorylation of $\mathrm{p} 21$ (Waf1/Cip1) as a novel mechanism of neuroprotection by glucocorticoids. The Journal of Neuroscience: The Official Journal of the Society for Neuroscience, 27(17), 4562-4571. http://doi.org/10.1523/JNEUROSCI.5110-06.2007

Hayes, W. K., Herbert, S. S. , Rehling, G. C. , \& Gennaro, J. F. (2002). Factors that influence venom expenditure in viperids and other snake species during predatory and defensive contexts: 207-233. In: Biology of the vipers. G. W. Schuett, M. Hoggren, M. E. Douglas, and H. W. Greene (eds.). Eagle Mountain Publ., Eagle Mountain, UT, USA.

Hertz, P. E., Huey, R. B., \& Nevo, E. (1982). Fight versus flight: Body temperature influences defensive responses of lizards. Animal Behaviour, 30(3). http://doi.org/10.1016/S0003-3472(82)80137-1

Hogan, M. P. (2015). Field venom extractions: saving fingers with tubes, forceps, and nerf bullets. Herpetological Review, 46(3), 339-342.

Holding, M. L., Owen, D. A. S., \& Taylor, E. N. (2014). Evaluating the thermal effects of translocation in a large-bodied pitviper. Journal of Experimental Zoology Part A: 
Ecological Genetics and Physiology, 321(A), 442-449.

http://doi.org/10.1002/jez.1876

Holding, M. L., Frazier, J. A., Dorr, S. W., Henningsen, S. N., Moore, I. T., \& Taylor, E. N. (2014a). Physiological and behavioral effects of repeated handling and shortdistance translocations on free-ranging Northern Pacific rattlesnakes ( Crotalus oreganus oreganus ). Journal of Herpetology, 48(2), 233-239. http://doi.org/10.1670/11-314

Holding, M. L., Frazier, J. A., Dorr, S. W., Pollock, N. B., Branske, A., Henningsen, S. N., ... Taylor, E. N. (2014b). Wet- and dry-season steroid hormone profiles and stress reactivity of an insular dwarf snake, the hog island boa ( Boa constrictor imperator ). Physiological and Biochemical Zoology, 87(3), 363-373. http://doi.org/10.1086/675938

Jessop, T. S., Hamann, M., Read, M. a, \& Limpus, C. J. (2000). Evidence for a hormonal tactic maximizing green turtle reproduction in response to a pervasive ecological stressor. General and Comparative Endocrinology, 118(3), 407-417. http://doi.org/10.1006/gcen.2000.7473

Jessop, T. S., Letnic, M., Webb, J. K., \& Dempster, T. (2013). Adrenocortical stress responses influence an invasive vertebrate's fitness in an extreme environment. Proceedings. Biological Sciences / The Royal Society, 280(1768), 20131444. http://doi.org/10.1098/rspb.2013.1444

Jones, S. M., \& Bell, K. (2004). Plasma corticosterone concentrations in males of the skink Egernia whitii during acute and chronic confinement, and over a diel period. 
Comparative Biochemistry and Physiology - A Molecular and Integrative

Physiology, 137(1), 105-113. http://doi.org/10.1016/S1095-6433(03)00267-8

Juneau, V., Gilmour, K., \& Blouin-Demers, G. (2015). Cocoa butter injections, but not sealed or perforated silastic implants, of corticosterone can be used to chronically elevate corticosterone in free-living painted turtles (Chrysemys picta). Journal of Herpetology, 49(4), 662-670.

Kabelik, D., Weiss, S. L., \& Moore, M. C. (2006). Steroid hormone mediation of limbic brain plasticity and aggression in free-living tree lizards, Urosaurus ornatus. Hormones and Behavior, 49(5), 587-597. http://doi.org/10.1016/j.yhbeh.2005.12.004

Kang, M., Ryu, J., Kim, J. H., Na, H., Zuo, Z., \& Do, S. H. (2010). Corticosterone decreases the activity of rat glutamate transporter type 3 expressed in Xenopus oocytes. Steroids, 75(13-14), 1113-1118. http://doi.org/10.1016/j.steroids.2010.07.003

Kempenaers, B., Peters, A., \& Foerster, K. (2008). Sources of individual variation in plasma testosterone levels. Philosophical Transactions of the Royal Society BBiological Sciences, 363(1497), 1711-1723. http://doi.org/10.1098/rstb.2007.0001

Keogh, S., \& DeSerto, F. P. (1994). Temperature Dependent Defensive Behavior in Three Species of North American Colubrid Snakes. Journal of Herpetology, 28(2), $258-261$.

Kini, R. M. (2003). Excitement ahead: Structure, function and mechanism of snake venom phospholipase A2 enzymes. Toxicon, 42(8), 827-840. 
http://doi.org/10.1016/j.toxicon.2003.11.002

Kini, R. M. (2005). Structure-function relationships and mechanism of anticoagulant phospholipase A2 enzymes from snake venoms. Toxicon, 45(8), 1147-1161. http://doi.org/10.1016/j.toxicon.2005.02.018

King, R. B. (2002). Family, sex and testosterone effects on garter snake behaviour. Animal Behaviour, 64(3), 345-359. http://doi.org/10.1006/anbe.2002.3046

Klauber, L.M., (1972). Rattlesnakes: Their Habits, Life Histories, and Influence on Mankind. University of California Press, Berkeley, CA, USA.

Landys, M. M., Ramenofsky, M., \& Wingfield, J. C. (2006). Actions of glucocorticoids at a seasonal baseline as compared to stress-related levels in the regulation of periodic life processes. General and Comparative Endocrinology, 148(2), 132-149. http://doi.org/10.1016/j.ygcen.2006.02.013

Lessells, C. M., \& Boag, P. T. (1987). Unrepeatable repeatabilities : a common mistake. The Auk, 104(1), 116-121. http://doi.org/10.2307/4087240

Li, X., Qiu, J., Wang, J., Zhong, Y., Zhu, J., \& Chen, Y. (2001). Corticosterone-induced rapid phosphorylation of p38 and JNK mitogen-activated protein kinases in PC12 cells. FEBS Letters, 492(3), 210-214. http://doi.org/10.1016/S0014-5793(01)022542

Lillywhite, H.B. (1987). Temperature, energetics, and physiological ecology. In Snakes: Ecology and Evolutionary Biology. Seigel, Collins, and Novak (Eds) pp. 422-477. Macmillan, New York, USA. 
Lind, C. M., Husak, J. F., Eikenaar, C., Moore, I. T., \& Taylor, E. N. (2010). The relationship between plasma steroid hormone concentrations and the reproductive cycle in the Northern Pacific rattlesnake, Crotalus oreganus. General and Comparative Endocrinology, 166(3), 590-599. http://doi.org/10.1016/j.ygcen.2010.01.026

Lind, C. M., \& Beaupre, S. J. (2015). Male Snakes Allocate Time and Energy according to Individual Energetic Status: Body Condition, Steroid Hormones, and Reproductive Behavior in Timber Rattlesnakes, Crotalus horridus. Physiological and Biochemical Zoology: Ecological and Evolutionary Approaches, 88(6), 624633. http://doi.org/10.1086/683058

Liu, W., Yuen, E. Y., \& Yan, Z. (2010). The stress hormone corticosterone increases synaptic alpha-amino-3- hydroxy-5-methyl-4-isoxazolepropionic acid (AMPA) receptors via serum- and glucocorticoid-inducible kinase (SGK) regulation of the GDI-Rab4 complex. Journal of Biological Chemistry, 285(9), 6101-6108. http://doi.org/10.1074/jbc.M109.050229

Lomonte, B., Angulo, Y., \& Calderón, L. (2003). An overview of lysine-49 phospholipase A2 myotoxins from crotalid snake venoms and their structural determinants of myotoxic action. Toxicon, 42(8), 885-901. http://doi.org/10.1016/j.toxicon.2003.11.008

Lorenz, C., Opitz, R., Lutz, I., \& Kloas, W. (2009). Corticosteroids disrupt amphibian metamorphosis by complex modes of action including increased prolactin expression. Comparative Biochemistry and Physiology, Part C, 150(2), 314-321. 
http://doi.org/10.1016/j.cbpc.2009.05.013

Lutterschmidt, D. I., \& Mason, R. T. (2010). Temporally distinct effects of stress and corticosterone on diel melatonin rhythms of red-sided garter snakes (Thamnophis sirtalis). General and Comparative Endocrinology, 169(1), 11-17. http://doi.org/10.1016/j.ygcen.2010.06.013

Lutterschmidt, W. I., Lutterschmidt, D. I., Mason, R. T., \& Reinert, H. K. (2009). Seasonal variation in hormonal responses of timber rattlesnakes (Crotalus horridus) to reproductive and environmental stressors. Journal of Comparative Physiology B, 179(6), 747-757. http://doi.org/10.1007/s00360-009-0356-2

Mackessy, S. P. (1988). Venom ontogeny in the Pacific rattlesnakes Crotalus viridis helleri and C. v. oreganus. Copeia, (1), 92-101.

Mackessy, S. P. (1996). Characterization of the major metalloprotease isolated from the venom of the Northern Pacific rattlesnake, Crotalus viridis oreganus. Toxicon, 34(11/12), 1277-1285.

Mackessy, S. P., Williams, K., \& Ashton, K. G. (2003). Ontogenetic variation in venom composition and diet of crotalus oreganus concolor : a case of venom paedomorphosis? Copeia, 2003(4), 769-782.

Margres, M., McGivern, J., Seavy, M., Wray, K., Facente, J., Rokyta, D. (2015).

Contrasting modes and tempos of venom expression evolution in two snake species. Genetics, 199, 165-176.

Margres, M., McGivern, J., Wray, K., Seavy, M., Calvin, K., Rokyta, D. (2014). Linking 
the transcriptome and proteome to characterize the venom of the eastern diamondback rattlesnake (Crotalus adamanteus). Journal of Proteomics, 96, $145-$ 158.

Maritz, B. (2012). To run or hide?: escape behaviour in a cryptic African snake. African Zoology, 47(2), 270-274. http://doi.org/10.3377/004.047.0215

Marqueti, R. C., Parizotto, N. A., Chriguer, S. R., Perez, S. E. A., \& Selistre-de-Araujo, H. S. (2006). Androgenic-anabolic steroids associated with mechanical loading inhibit matrix metallopeptidase activity and affect the remodeling of the achilles tendon in rats. The American Journal of Sports Medicine, 34(8), 1274-1280.

Martin, L. B. (2009). Stress and immunity in wild vertebrates: Timing is everything. General and Comparative Endocrinology, 163(1-2), 70-76. http://doi.org/10.1016/j.ygcen.2009.03.008

Massey, D. J., Calvete, J. J., Sánchez, E. E., Sanz, L., Richards, K., Curtis, R., \& Boesen, K. (2012). Venom variability and envenoming severity outcomes of the Crotalus scutulatus scutulatus (Mojave rattlesnake) from Southern Arizona. Journal of Proteomics, 75(9), 2576-2587. http://doi.org/10.1016/j.jprot.2012.02.035

McCormick, G. L., \& Langkilde, T. (2014). Immune responses of eastern fence lizards (Sceloporus undulatus) to repeated acute elevation of corticosterone. General and Comparative Endocrinology, 204(May), 135-140. http://doi.org/10.1016/j.ygcen.2014.04.037

McCue, M. D. (2007). Western diamondback rattlesnakes demonstrate physiological and biochemical strategies for tolerating prolonged starvation. Physiological and 
Biochemical Zoology, 80(1), 25-34. http://doi.org/10.1086/509057

Miles, D. B., Calsbeek, R., \& Sinervo, B. (2007). Corticosterone, locomotor performance, and metabolism in side-blotched lizards (Uta stansburiana). Hormones and Behavior, 51(4), 548-554. http://doi.org/10.1016/j.yhbeh.2007.02.005

Moore, I. T., Greene, M. J., \& Mason, R. (2001). Environmental and seasonal adaptations of the adrenocortical and gonadal responses to capture stress in two populations of the male garter snake, Thamnophis sirtalis. Journal of Experimental Zoology, 289(2), 99-108. http://doi.org/10.1002/1097-010X(20010201)289:2<99::AIDJEZ3>3.0.CO;2-Z

Moore, I. T., Lemaster, M. P., \& Mason, R. T. (2001). Behavioural and hormonal responses to capture stress in the male red-sided garter snake, Thamnophis sirtalis parietalis. Physiology \& Behavior, 72, 669-674. http://doi.org/10.1006/anbe.1999.1344

Moore, I. T., Lerner, J. P., Lerner, D. T., \& Mason, R. T. (2000). Relationships between annual cycles of testosterone, corticosterone, and body condition in male red-spotted garter snakes, Thamnophis sirtalis concinnus. Physiological and Biochemical Zoology : PBZ, 73(3), 307-312. http://doi.org/10.1086/316748

Moore, M. C. (1987). Castration affects territorial and sexual behaviour of free-living male lizards, Sceloporus jarrovi. Animal Behaviour, 35(4), 1193-1199. http://doi.org/10.1016/S0003-3472(87)80176-8

Morris, D. J., Latif, S. A., \& Brem, A. S. (2009). Interactions of mineralocorticoids and glucocorticoids in epithelial target tissues revisited. Steroids, 74(1), 1-6. 
http://doi.org/10.1016/j.steroids.2008.10.005

Müller, C., Almasi, B., Roulin, A., Breuner, C. W., Jenni-Eiermann, S., \& Jenni, L. (2009). Effects of corticosterone pellets on baseline and stress-induced corticosterone and corticosteroid-binding-globulin. General and Comparative Endocrinology, 160(1), 59-66. http://doi.org/10.1016/j.ygcen.2008.10.018

Murphy, J. B. (2014). Studies on venomous reptiles in zoos and aquariums. Part I. Introduction, rear-fanged colubrid snakes, elapids, sea kraits and seasnakes. Herpetological Review, 45(1), 158-178.

Narayan, E. J., \& Hero, J. M. (2013). Repeatability of baseline corticosterone and acute stress responses to capture, and patterns of reproductive hormones in vitellogenic and non-vitellogenic female fijian ground frog (platymantis vitiana). Journal of Experimental Zoology Part A: Ecological Genetics and Physiology, 319(8), 471481. http://doi.org/10.1002/jez.1810

Neuman-Lee, L., Fokidis, H., Spence, A., Van der Walt, G., Durham, S. \& French, S. (2015). Food restriction and chronic stress alter energy use and affect immunity in an infrequent feeder. Functional Ecology,29, 1453-1462.

Núñez, V., Cid, P., Sanz, L., De La Torre, P., Angulo, Y., Lomonte, B., Gutiérrez, J., Calvete, J. (2009). Snake venomics and antivenomics of Bothrops atrox venoms from Colombia and the Amazon regions of Brazil, Perú and Ecuador suggest the occurrence of geographic variation of venom phenotype by a trend towards paedomorphism. Journal of Proteomics 73, 57-78.

Oksanen, J., Blanchet, F., Kindt, R., Legendre, P., Minchin, P., O'Hara, R., Simpson, G., 
Solymos, P., Stevens, M., Wagner, H. \& Oksanen, M. (2013). Package 'vegan'. Community ecology package, version, 2(9).

Pigliucci, M. (2001). Phenotypic Plasticity: Beyond Nature and Nurture. Baltimore: Maryland, USA: Johns Hopkins University Press

Preest, M. R., \& Cree, A. (2008). Corticosterone treatment has subtle effects on thermoregulatory behavior and raises metabolic rate in the New Zealand common gecko, Hoplodactylus maculatus. Physiological and Biochemical Zoology, 81(5), 641-650. http://doi.org/10.1086/590371

Press, A. (2016). Relations of Body Temperature, Size, and Crawling Speed of the Common Garter Snake, Thamnophis s . sirtalis Author ( s ): Carlton Heckrotte Published by : American Society of Ichthyologists and Herpetologists ( ASIH ) Stable URL : http://www.jstor.org/, 1967(4), 759-763.

Putman, B. J., Lind, C., \& Taylor, E. N. (2013). Does Size Matter? Factors Influencing the Spatial Ecology of Northern Pacific Rattlesnakes (Crotalus oreganus oreganus) in Central California. Copeia, 2013(3), 485-492. http://doi.org/10.1643/ce-12-048

Rich, E. L., \& Romero, L. M. (2005). Exposure to chronic stress downregulates corticosterone responses to acute stressors. American Journal of Physiology. Regulatory, Integrative and Comparative Physiology, 288(6), R1628-R1636. http://doi.org/10.1152/ajpregu.00484.2004

Rodgers, A. R., Carr, A.P., Beyer, H.L., Smith, L., Kie, J.G. (2007). HRT: home range tools forArcGIS. 
Rokyta, D. R., Margres, M. J., \& Calvin, K. (2015). Post-transcriptional mechanisms contribute little to phenotypic variation in snake venoms. G3: Genes Genomes Genetics, 5, 2375-2382. http://doi.org/10.1534/g3.115.020578

Romero, L. M., \& Reed, J. M. (2005). Collecting baseline corticosterone samples in the field: Is under 3 min good enough? Comparative Biochemistry and Physiology - A Molecular and Integrative Physiology, 140(1), 73-79. http://doi.org/10.1016/j.cbpb.2004.11.004

Romero, L. M., Strochlic, D., \& Wingfield, J. C. (2005). Corticosterone inhibits feather growth: Potential mechanism explaining seasonal down regulation of corticosterone during molt. Comparative Biochemistry and Physiology Part A: Molecular \& Integrative Physiology, 142(1), 65-73. http://doi.org/10.1016/j.cbpa.2005.07.014

Romero, L. M., \& Wikelski, M. (2006). Diurnal and nocturnal differences in hypothalamic-pituitary-adrenal axis function in Gal??pagos marine iguanas. General and Comparative Endocrinology, 145(2), 177-181. http://doi.org/10.1016/j.ygcen.2005.09.011

Salazar, A. M., Guerrero, B., Cantu, B., Cantu, E., Rodríguez-acosta, A., Pérez, J. C., ... Sánchez, E. E. (2009). Venom variation in hemostasis of the southern Pacific rattlesnake (Crotalus oreganus helleri ): Isolation of hellerase. Comparative Biochemistry and Physiology, Part C, 149(3), 307-316.

http://doi.org/10.1016/j.cbpc.2008.08.007

Salvante, K. G., \& Williams, T. D. (2003). Effects of corticosterone on the proportion of breeding females, reproductive output and yolk precursor levels. General and 
Comparative Endocrinology, 130(3), 205-214. http://doi.org/10.1016/S00166480(02)00637-8

Sapolsky, R. M., Romero, L. M., \& Munck, a. U. (2000). How do glucocorticoids influence stress responses? Integrating permissive, suppressive, stimulatory, and preparative actions *. Endocrine Reviews, 21(April), 55-89. http://doi.org/10.1210/er.21.1.55

Saviola, A. J., Pla, D., Sanz, L., Castoe, T. A., Calvete, J. J., \& Mackessy, S. P. (2015). Comparative venomics of the Prairie Rattlesnake ( Crotalus viridis viridis ) from Colorado : Identification of a novel pattern of ontogenetic changes in venom composition and assessment of the immunoreactivity of the commercial antivenom CroFab. Journal of Proteomics, 121, 28-43. http://doi.org/10.1016/j.jprot.2015.03.015

Schuett, G. W., Harlow, H. J., Rose, J. D., Van Kirk, E. a, \& Murdoch, W. J. (1996). Levels of plasma corticosterone and testosterone in male copperheads (Agkistrodon contortrix) following staged fights. Hormones and Behavior, 30(1), 60-68. http://doi.org/10.1006/hbeh.1996.0009

Schuett, G. W., \& Grober, M. S. (2000). Post-fight levels of plasma lactate and corticosterone in male copperheads, Agkistrodon contortrix (Serpentes, Viperidae): Differences between winners and losers. Physiology and Behavior, 71(3-4), 335341. http://doi.org/10.1016/S0031-9384(00)00348-6

Schuett, G. W., Taylor, E. N., Van Kirk, E. A., \& Murdoch, W. J. (2004). Handling Stress and Plasma corticosterone levels in captive male Western diamond-backed 
rattlesnakes ( Crotalus atrox ). Herpetological Review, 35(3), 229-233.

Seddon, R. J., \& Klukowski, M. (2012). Influence of Stressor Duration on Leukocyte and Hormonal Responses in Male Southeastern Five-Lined Skinks (Plestiodon inexpectatus). Journal of Experimental Zoology Part A: Ecological Genetics and Physiology, 317(8), 499-510. http://doi.org/10.1002/jez.1742

Seebacher, F. (2005). A review of thermoregulation and physiological in reptiles: what is the role of phenotypic flexibility? Journal of Comparative Physiology. B, Biochemical, Systemic, and Environmental Physiology, 175(7), 453-61. http://doi.org/10.1007/s00360-005-0010-6

Shikatani, E. A., Trifonova, A., Mandel, E. R., Liu, S. T. K., Roudier, E., Krylova, A., ... Haas, T. L. (2012). Inhibition of proliferation, migration and proteolysis contribute to corticosterone-mediated inhibition of angiogenesis. PloS One, 7(10). http://doi.org/10.1371/journal.pone.0046625

Shukla, P. K., Gautam, L., Sinha, M., Kaur, P., Sharma, S., \& Singh, T. P. (2015). Structures and binding studies of the complexes of phospholipase A2 with five inhibitors. BBA - Proteins and Proteomics, 1854(4), 269-277. http://doi.org/10.1016/j.bbapap.2014.12.017

Simpson, I., \& Jacobsen, I. (2009). Antisnake venom production crisis--who told us it was uneconomic and unsustainable? Wilderness \& Environmental Medicine, 20(2), 144-55.

Sparkman, A. M., Bronikowski, A. M., Williams, S., Parsai, S., Manhart, W., \& Palacios, M. G. (2014). Physiological indices of stress in wild and captive garter snakes: 
Correlations, repeatability, and ecological variation. Comparative Biochemistry and Physiology-Part A : Molecular and Integrative Physiology, 174, 11-17. http://doi.org/10.1016/j.cbpa.2014.03.023

Spée, M., Marchal, L., Lazin, D., Le Maho, Y., Chastel, O., Beaulieu, M., \& Raclot, T. (2011). Exogenous corticosterone and nest abandonment: A study in a long-lived bird, the Adélie penguin. Hormones and Behavior, 60(4), 362-370. http://doi.org/10.1016/j.yhbeh.2011.07.003

Spencer, M. M., Lardner, B., Mazurek, M., \& Reed, R. N. (2012). Factors affecting defensive strike behavior in brown treesnakes (Boiga irregularis) provoked by humans. Herpetological Conservation and Biology, 10(2), 703-710.

Sperry, J. H., \& Weatherhead, P. J. (2008). Prey-mediated effects of drought on condition and survival of a terrestrial snake. Ecology, 89(10), 2770-2776. http://doi.org/10.1890/07-2017.1

Sunagar, K., Undheim, E. a B., Scheib, H., Gren, E. C. K., Cochran, C., Person, C. E., ... Fry, B. G. (2014). Intraspecific venom variation in the medically significant Southern Pacific Rattlesnake (Crotalus oreganus helleri): Biodiscovery, clinical and evolutionary implications. Journal of Proteomics, 99(July), 68-83. http://doi.org/10.1016/j.jprot.2014.01.013

Sykes, K. L., \& Klukowski, M. (2009). Effects of acute temperature change, confinement and housing on plasma corticosterone in water snakes, Nerodia sipedon (Colubridae: Natricinae). Journal of Experimental Zoology Part A: Ecological Genetics and Physiology, 311(3), 172-181. http://doi.org/10.1002/jez.515 
Taylor, E. N., Denardo, D. F., \& Jennings, D. H. (2004). Seasonal steroid hormone levels and their relation to reproduction in the Western Diamond-backed Rattlesnake, Crotalus atrox (Serpentes: Viperidae). General and Comparative Endocrinology, 136(3), 328-337. http://doi.org/10.1016/j.ygcen.2004.01.008

Templ, M., Filzmoser, P., \& Hron, K. (2011). Robust imputation of missing values in compositional data using the R-package robCompositions. New Techniques and Technologies for Statistics, Brussels.

Thunhorst, R. L., Beltz, T. G., \& Johnson, A. K. (2007). Glucocorticoids increase salt appetite by promoting water and sodium excretion. American Journal of Physiology. Regulatory, Integrative and Comparative Physiology, 293(3), R1444-R1451. http://doi.org/10.1152/ajpregu.00294.2007

Tyrrell, C. L., \& Cree, A. (1998). Relationships between corticosterone concentration and season, time of day and confinement in a wild reptile (tuatara, Sphenodon punctatus). Gen Comp Endocr, 110(2), 97-108. http://doi.org/10.1006/gcen.1997.7051

Vishwanath, B. S., Frey, F. J., Bradbury, M. J., Dallman, M. F., \& Frey, B. M. (1993). Glucocorticoid deficiency increases phospholipase A2 activity in rats. The Journal of Clinical Investigation, 92(4), 1974-1980. http://doi.org/10.1172/JCI116791

Wack, C. L., DuRant, S. E., Hopkins, W. a., Lovern, M. B., Feldhoff, R. C., \& Woodley, S. K. (2012). Elevated plasma corticosterone increases metabolic rate in a terrestrial salamander. Comparative Biochemistry and Physiology - A Molecular and Integrative Physiology, 161(2), 153-158. http://doi.org/10.1016/j.cbpa.2011.10.017 
Wada, H. (2008). Glucocorticoids: mediators of vertebrate ontogenetic transitions.

General and Comparative Endocrinology, 156(3), 441-53.

http://doi.org/10.1016/j.ygcen.2008.02.004

Wasko, D. K., \& Sasa, M. (2009). Activity Patterns of a Neotropical Ambush Predator : Spatial Ecology of the Fer-de-lance ( Bothrops asper, Serpentes : Viperidae ) in Costa Rica. Biotropica, 41(2), 241-249.

Wasserberger, J., Ordog, G., \& Merkin, T. (2006). Southern Pacific rattlesnake bite : a unique clinical challenge. The Journal of Emergency Medicine, 31(3), 263-266. http://doi.org/10.1016/j.jemermed.2005.09.018

While, G. M., Isaksson, C., McEvoy, J., Sinn, D. L., Komdeur, J., Wapstra, E., \& Groothuis, T. G. G. (2010). Repeatable intra-individual variation in plasma testosterone concentration and its sex-specific link to aggression in a social lizard. Hormones and Behavior, 58(2), 208-213. http://doi.org/10.1016/j.yhbeh.2010.03.016

Whitaker, R., \& Whitaker, S. (2012). Venom, antivenom production and the medically important snakes of India. Current Science, 103(6), 635-643.

Willemse, G. ., Hattingh, J., Karlsson, R. M., Levy, S., \& Parker, C. (1979). Changes in composition and protein concentration of puff adder (Bitis arietans) venom due to frequent milking. Toxicon, 17, 37-42.

Wingfield, J. C. (2005). The Concept of Allostasis: Coping With a Capricious Environment. Journal of Mammalogy, 86(2), 248-254. http://doi.org/10.1644/BHE004.1 
Wingfield, J. C., \& Sapolsky, R. M. (2003). Reproduction and resistance to stress: when and how. Journal of Neuroendocrinology, 15(8), 711-724. http://doi.org/10.1046/j.1365-2826.2003.01033.x

Wingfield, J. C. (2013). Ecological processes and the ecology of stress: The impacts of abiotic environmental factors. Functional Ecology, 27(1), 37-44. http://doi.org/10.1111/1365-2435.12039

Woodley, S. K., Painter, D. L., Moore, M. C., Wikelski, M., \& Michael Romero, L. (2003). Effect of tidal cycle and food intake on the baseline plasma corticosterone rhythm in intertidally foraging marine iguanas. General and Comparative Endocrinology, 132(2), 216-222. http://doi.org/10.1016/S0016-6480(03)00085-6

Yañez-Arenas, C., Townsend Peterson, A., Rodríguez-Medina, K., \& Barve, N. (2016). Mapping current and future potential snakebite risk in the new world. Climatic Change, 134, 697-711. http://doi.org/10.1007/s10584-015-1544-6

Yang, E. J., \& Wilczynski, W. (2003). Interaction effects of corticosterone and experience on aggressive behavior in the green anole lizard. Hormones and Behavior, 44(3), 281-292. http://doi.org/10.1016/S0018-506X(03)00139-9 


\section{APPENDICES}

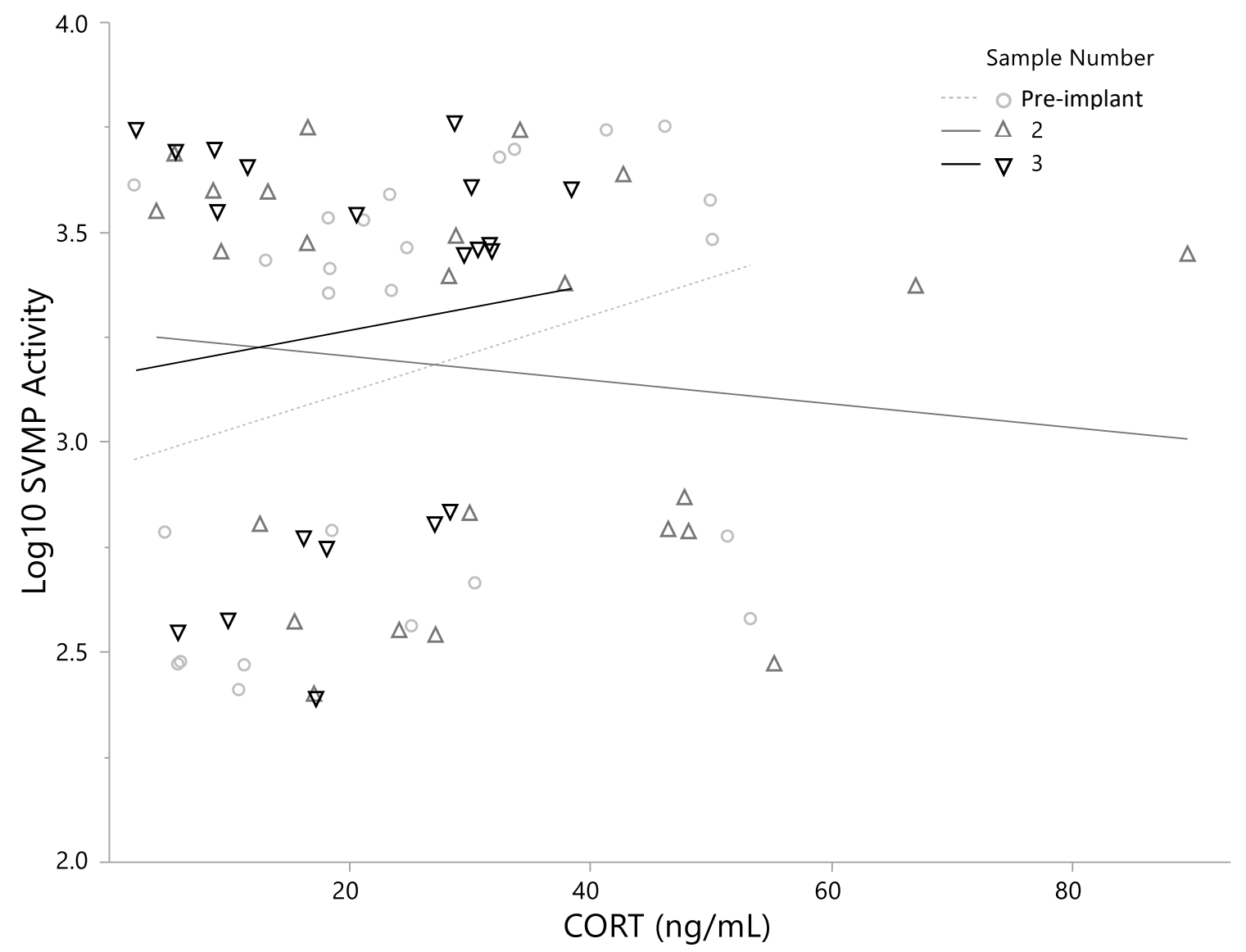

Figure A1. $\log _{10}$ transformed SVMP activity of Crotalus helleri plotted against baseline CORT values across all three sampling periods (coded sequentially from light to dark). There was no relationship between baseline sampled CORT and SVMP activity in the RM ANCOVA.

Although all three samples from each snake are shown, each within-snake sample was not considered independent. The RM ANCOVA accounted for repetition within individuals, so there was no pseudoreplication in our dataset. 


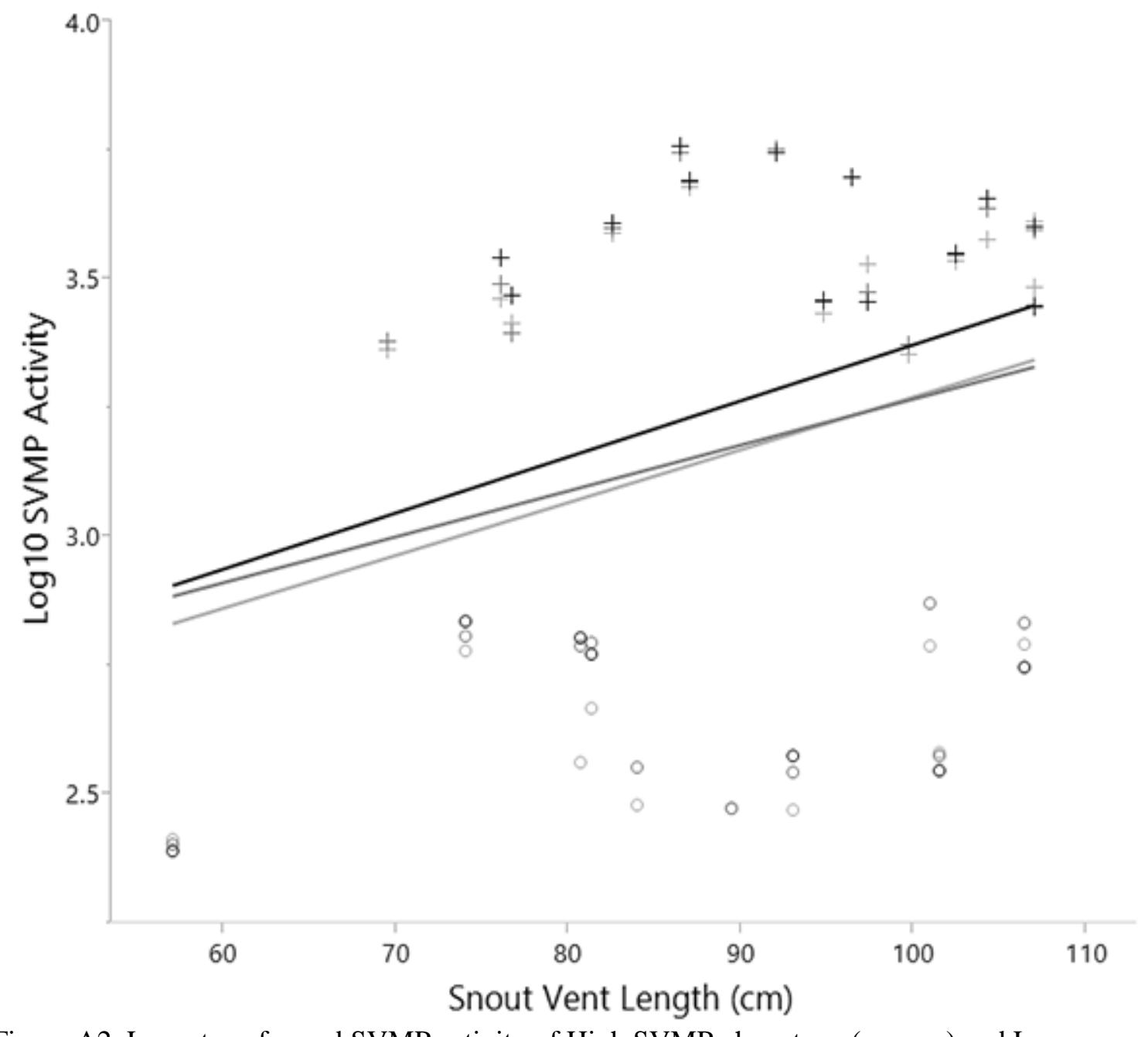

Figure A2. $\log _{10}$ transformed SVMP activity of High SVMP phenotype (crosses) and Low SVMP phenotype (open circles) Crotalus helleri across the three sampling periods (in sequence from light to dark). SVL was not a significant covariate in the full model. Note opposing pattern between phenotypes and SVL. Note also that clusters of points along the $\mathrm{x}$-axis represent the samples from the same individual snake as SVL did not change appreciably during the study. Although all three samples from each snake are shown, each within-snake sample was not considered independent. The RM ANCOVA accounted for repetition within individuals, so there was no pseudoreplication in our dataset. 


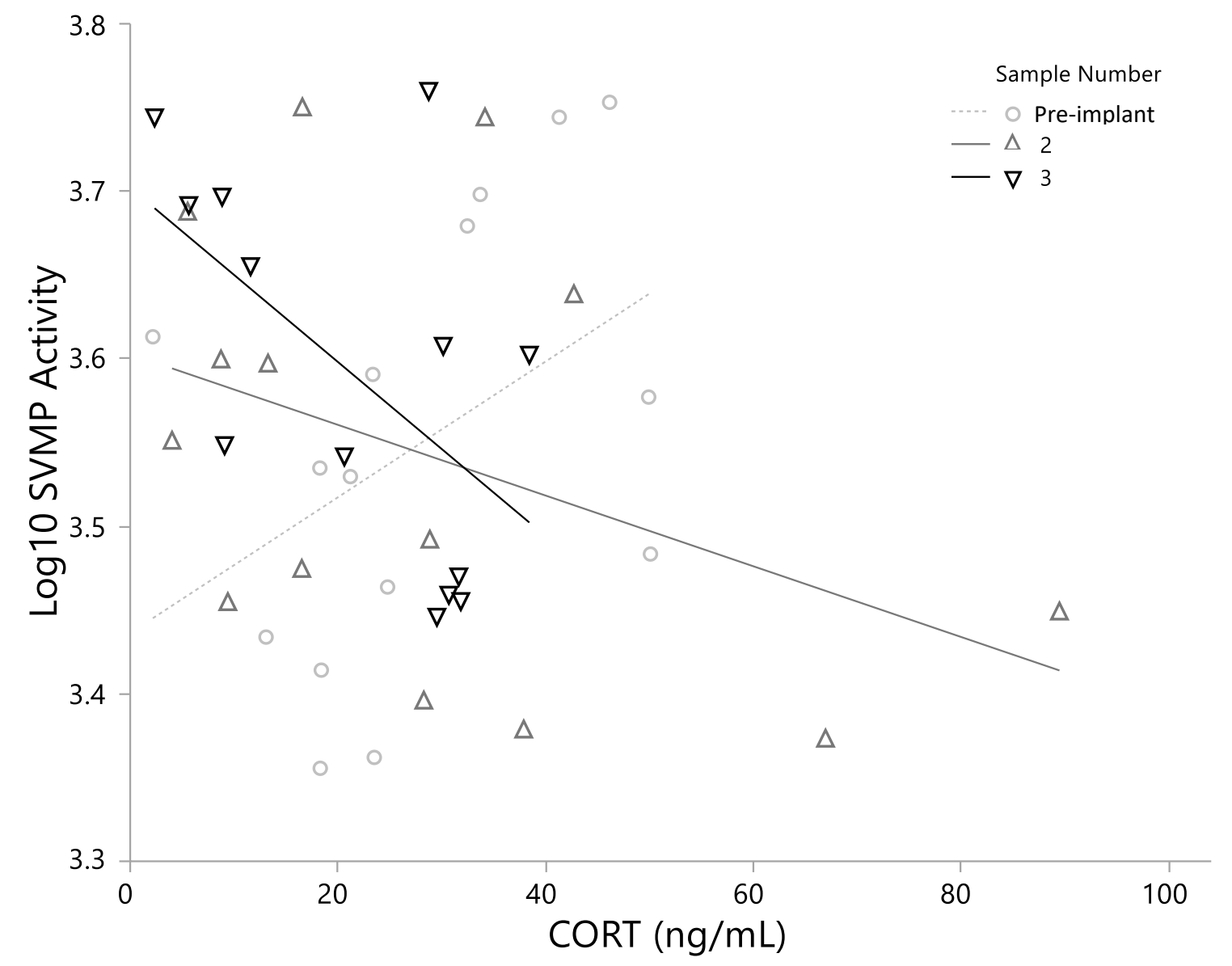

Figure A3. $\log _{10}$ transformed SVMP activity of High SVMP activity phenotype Crotalus helleri plotted against baseline CORT values across all three sampling periods (in sequence from light to dark). CORT was not a significant covariate. Although all three samples from each snake are shown, each within-snake sample was not considered independent. The RM ANCOVA accounted for repetition within individuals, so there was no pseudoreplication in our dataset. 


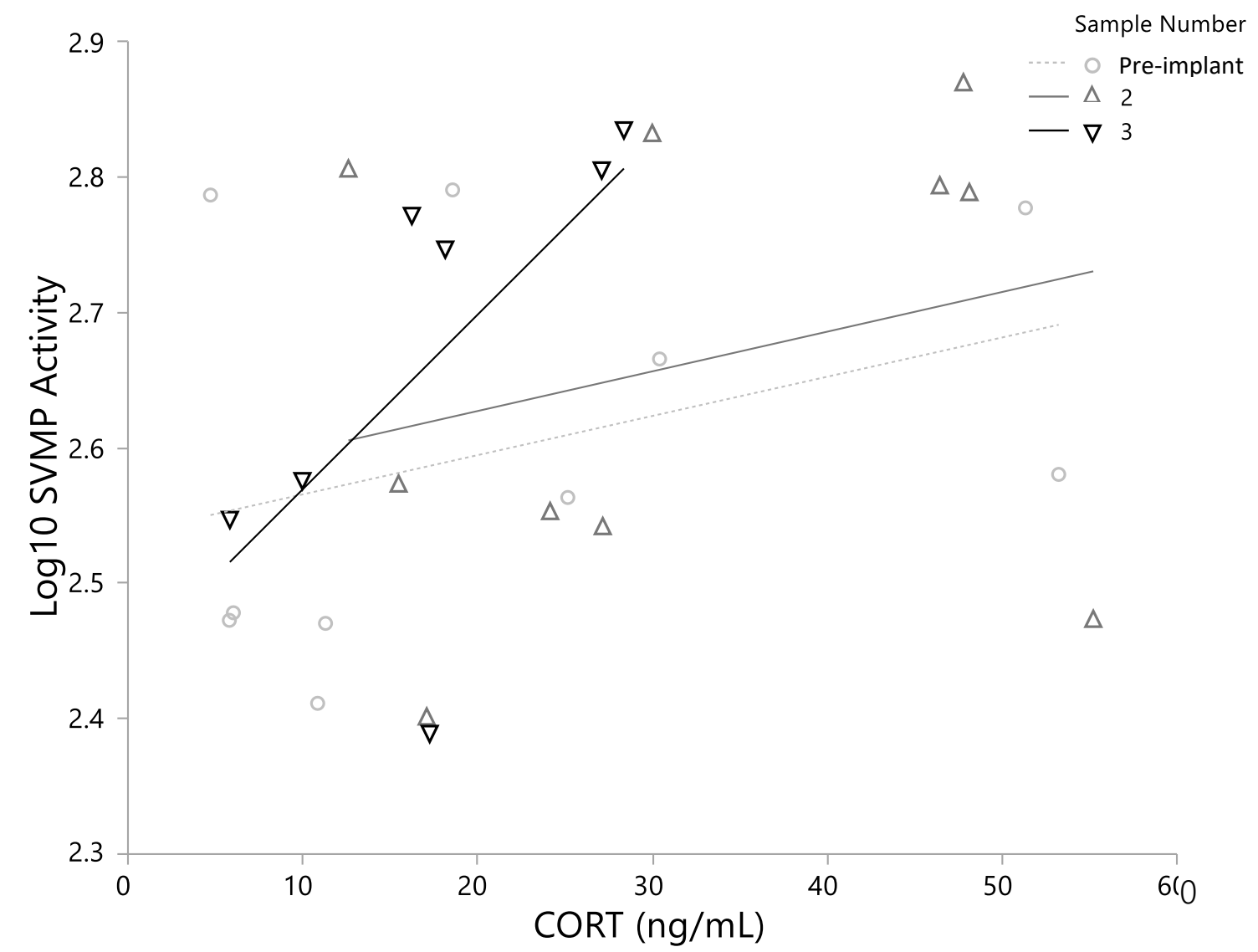

Figure A4. $\log _{10}$ transformed SVMP activity of Low SVMP activity phenotype Crotalus helleri plotted against baseline CORT values across all three sampling periods (in sequence from light to dark). CORT was not a significant covariate. Although all three samples from each snake are shown, each within-snake sample was not considered independent. The RM ANCOVA accounted for repetition within individuals, so there was no pseudoreplication in our dataset. 


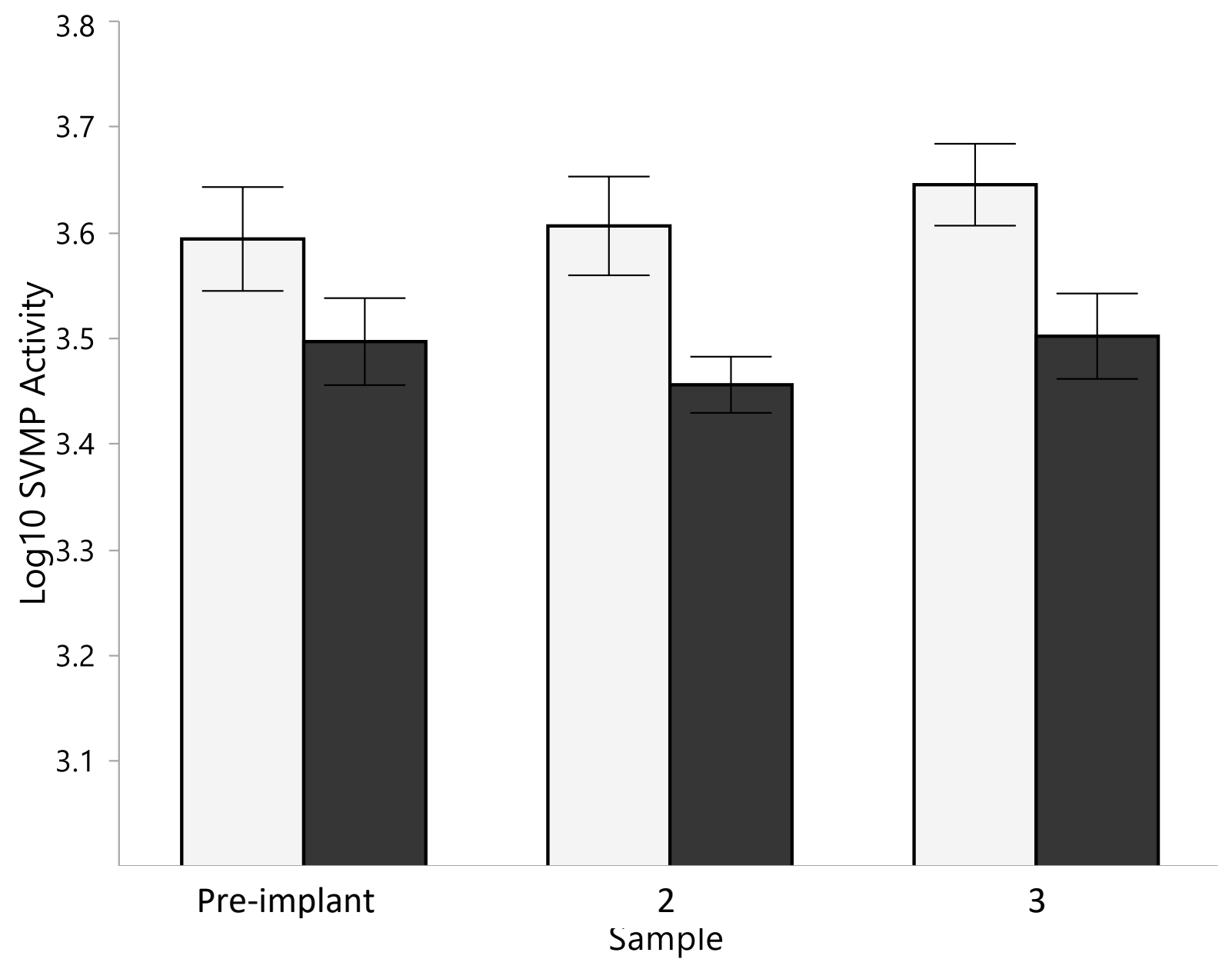

Figure A5. Mean $\log _{10}$ transformed SVMP activity of High SVMP Phenotype control (light bars) and CORT-implanted (dark bars) Crotalus helleri. There was no effect of treatment or repeated sampling in the High SVMP Phenotype group on SVMP activity. Error bars are \pm 1 SEM. 


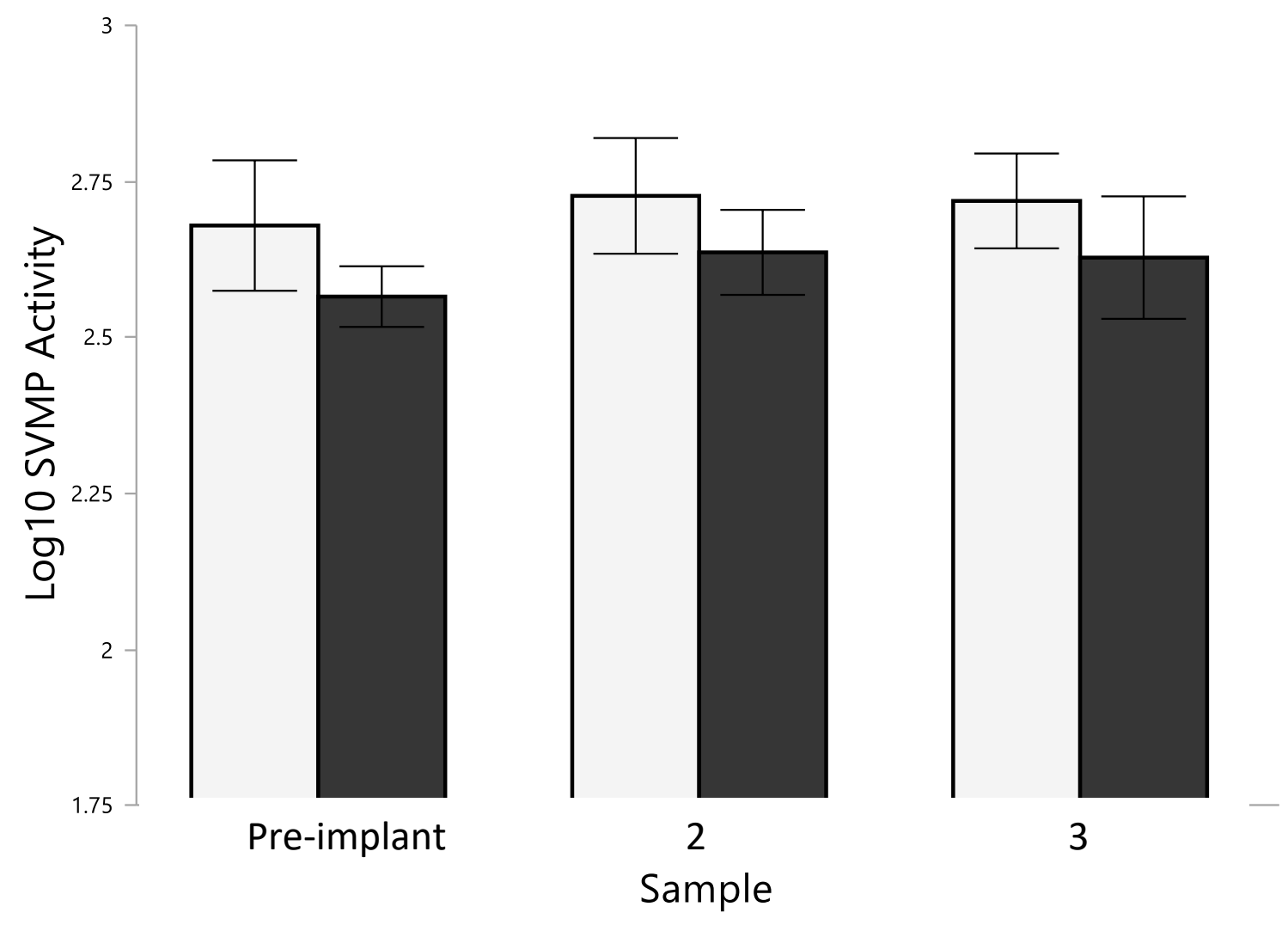

Figure A6. Mean $\log _{10}$ transformed SVMP activity of Low SVMP Phenotype control (light bars) and CORT-implanted (dark bars) Crotalus helleri. There was no effect of treatment or repeated sampling in the Low SVMP Phenotype group on SVMP activity.

Error bars are \pm 1 SEM. 


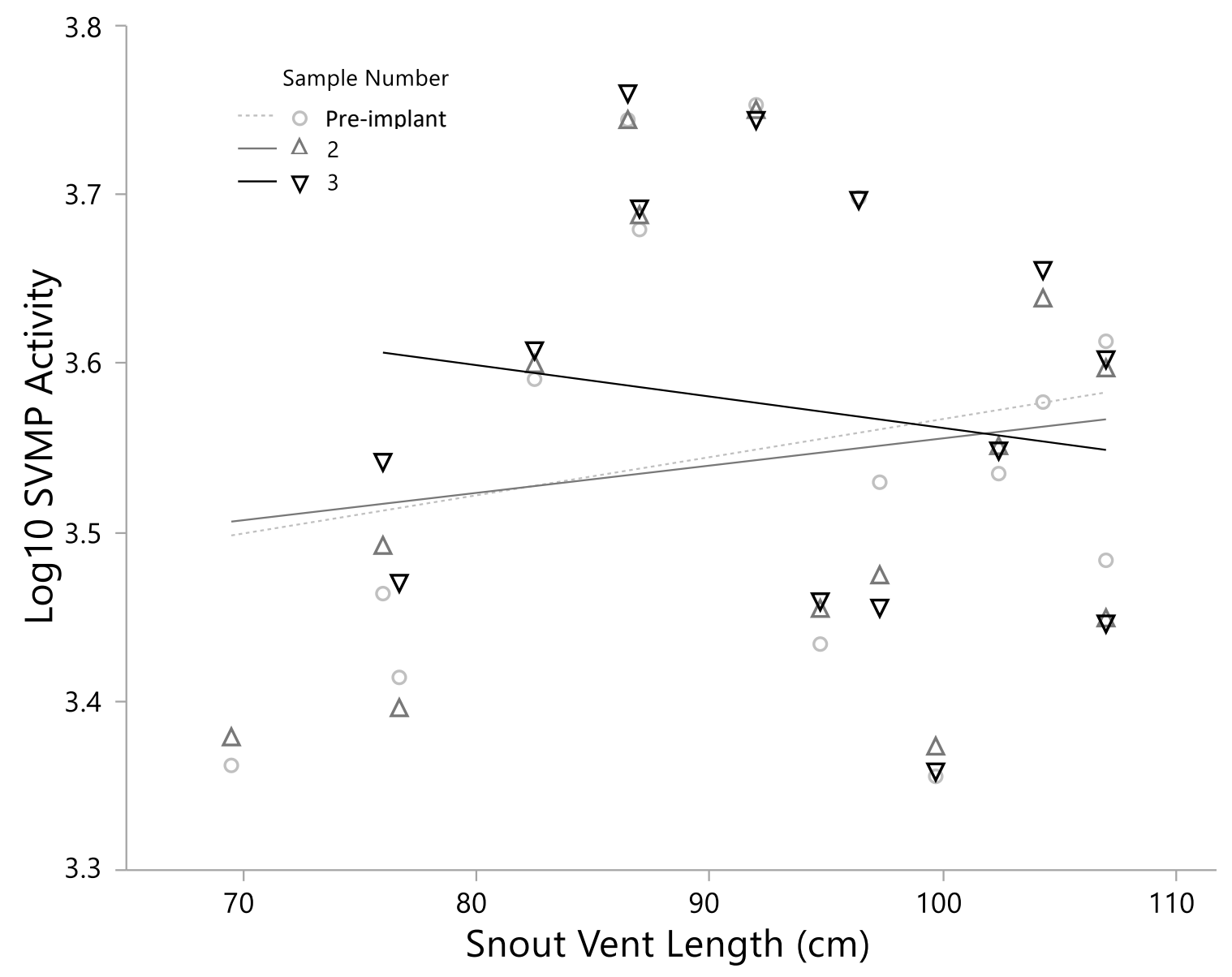

Figure A7. $\log _{10}$ transformed SVMP activity of High SVMP activity phenotype Crotalus helleri plotted against SVL values across all three sampling periods. SVL was a significant covariate in the RM ANCOVA, with larger High Phenotype snakes tending to have higher SVMP activity. Although all three samples from each snake are shown, each within-snake sample was not considered independent. The RM ANCOVA accounted for repetition within individuals, so there was no pseudoreplication in our dataset. 


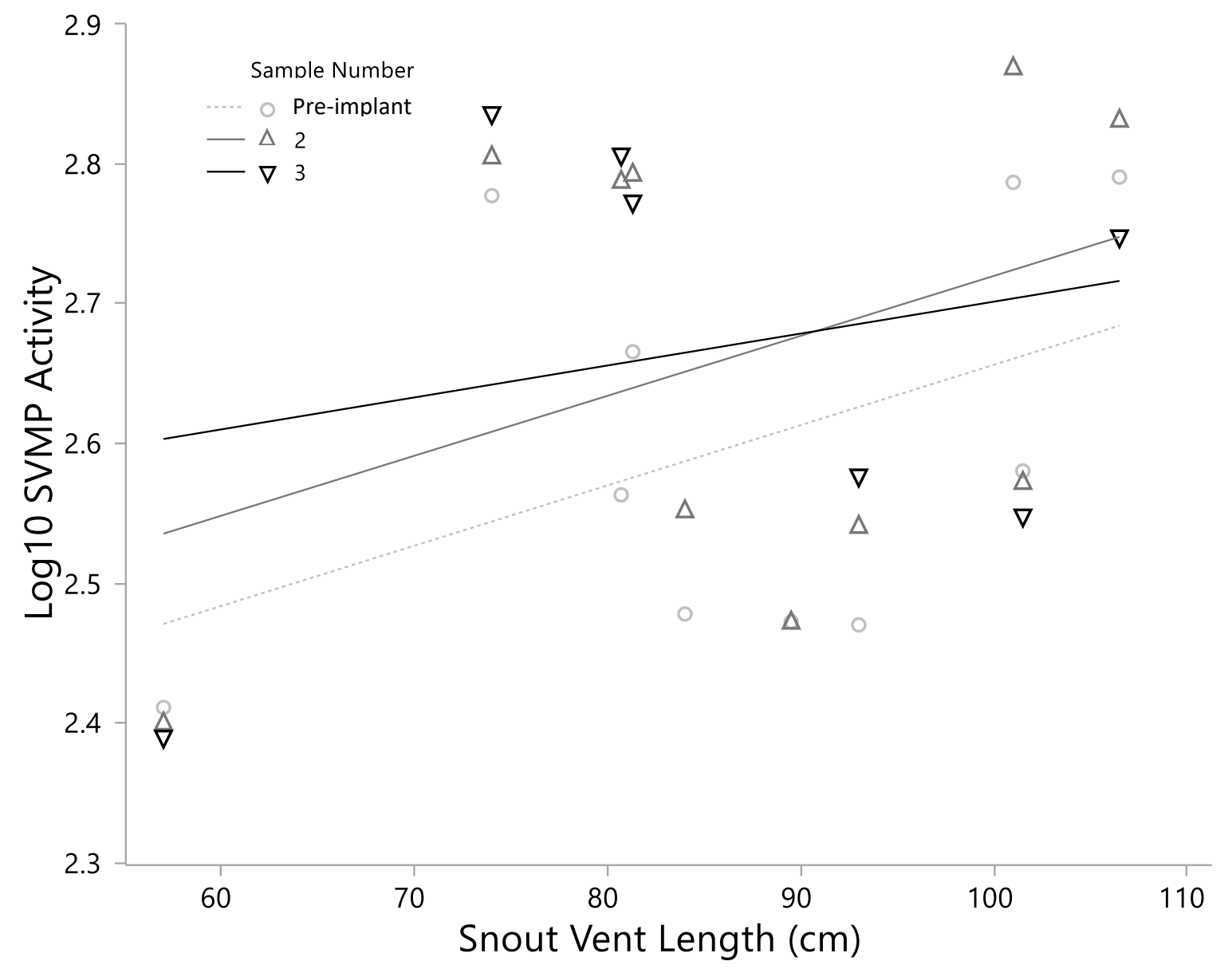

Figure A8. $\log _{10}$ transformed SVMP activity of Low SVMP activity phenotype Crotalus helleri plotted against SVL across all three sampling periods (in sequence from light to dark). SVL was not a significant covariate. Although all three samples from each snake are shown, each withinsnake sample was not considered independent. The RM ANCOVA accounted for repetition within individuals, so there was no pseudoreplication in our dataset. 


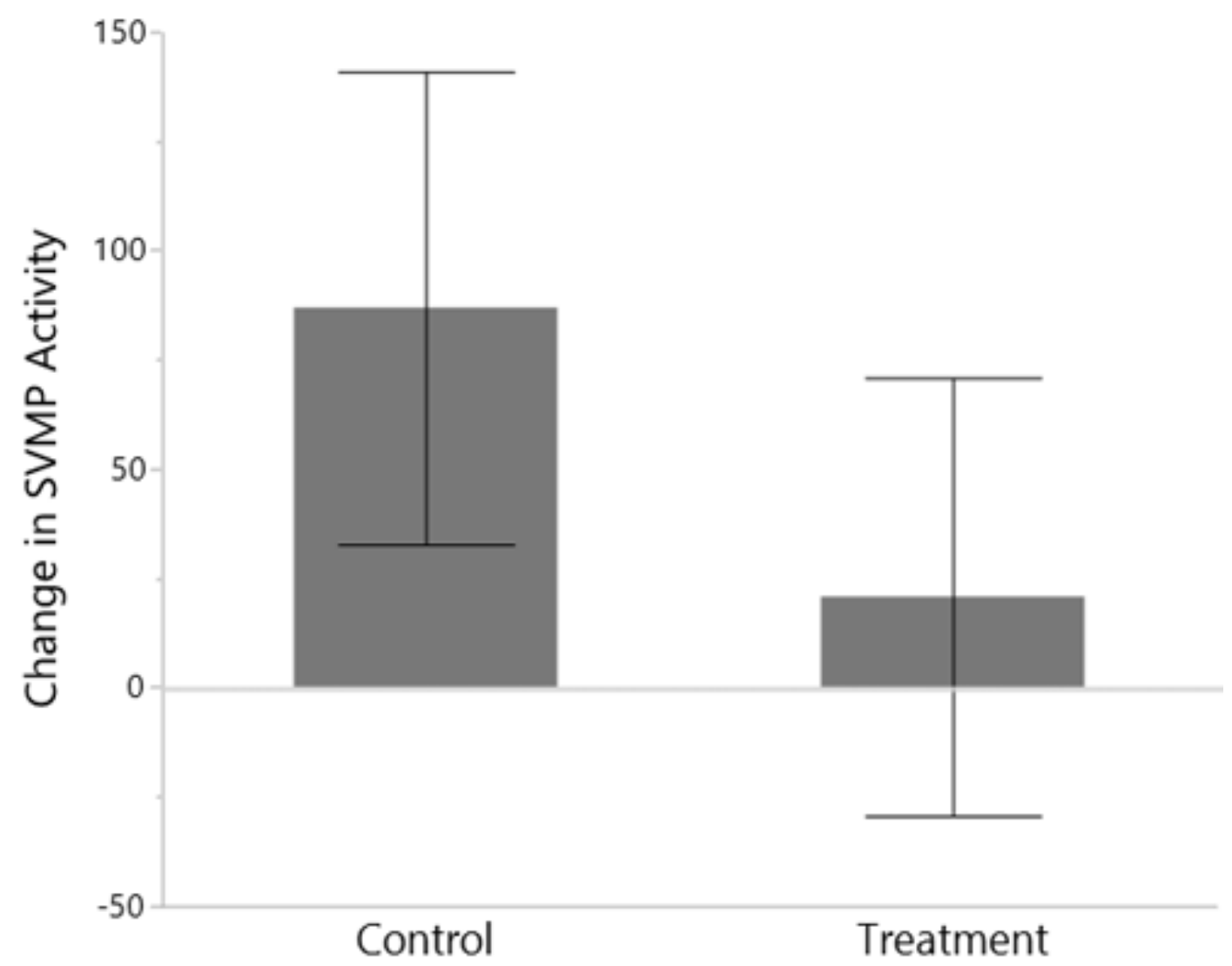

Figure A9. Mean change in SVMP activity from pre-implant sampling to sample 2 in control and CORT-implanted Crotalus helleri. Both control and treatment snakes showed overall average increases in SVMP activity during the initial implant period. There was no effect of treatment on change in SVMP activity in an ANCOVA. Error bars are \pm 1 SEM. 


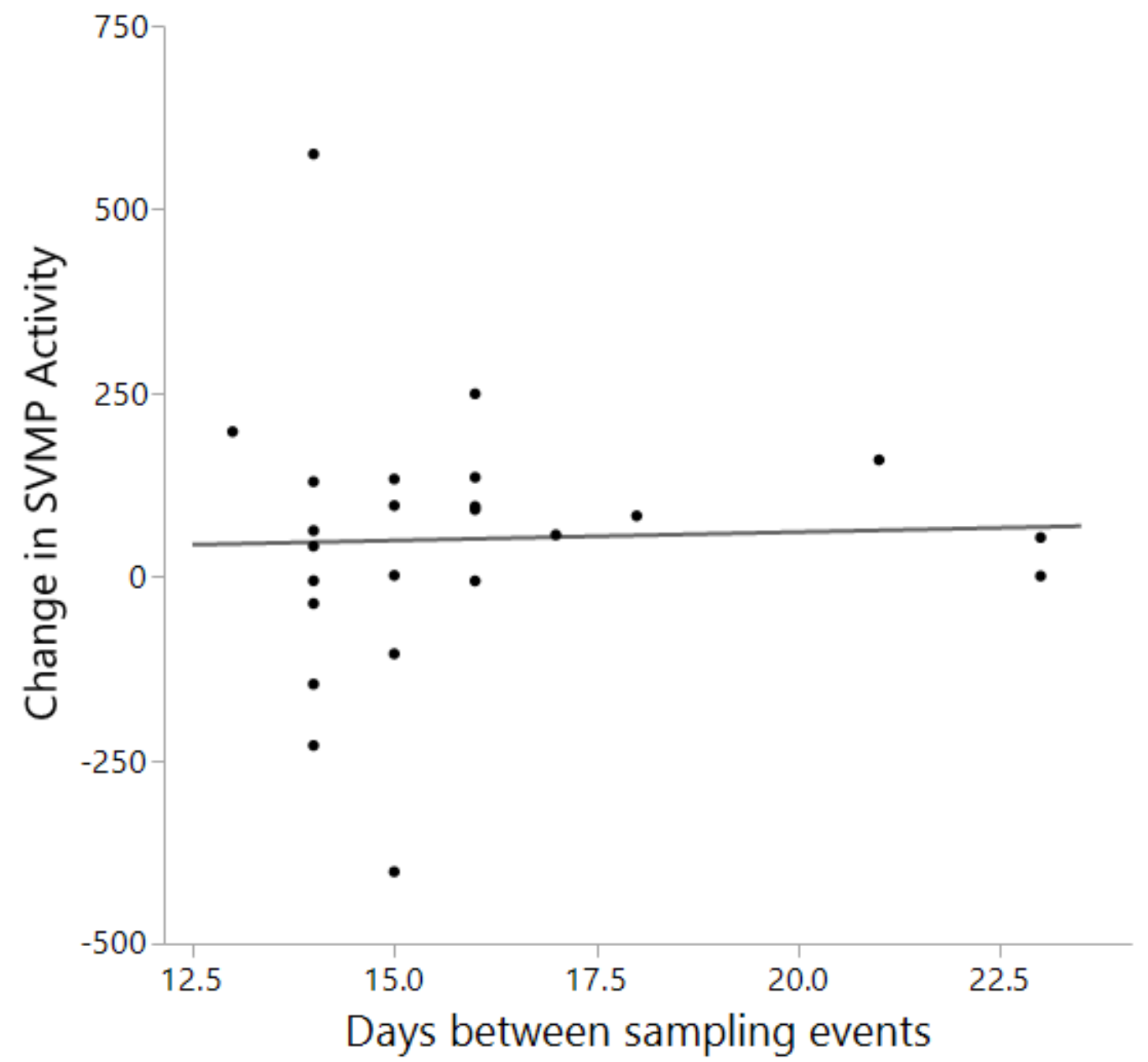

Figure A10. Change in SVMP activity from pre-implant sampling to sample 2 in Crotalus helleri. Days between sampling events was not a significant covariate. 


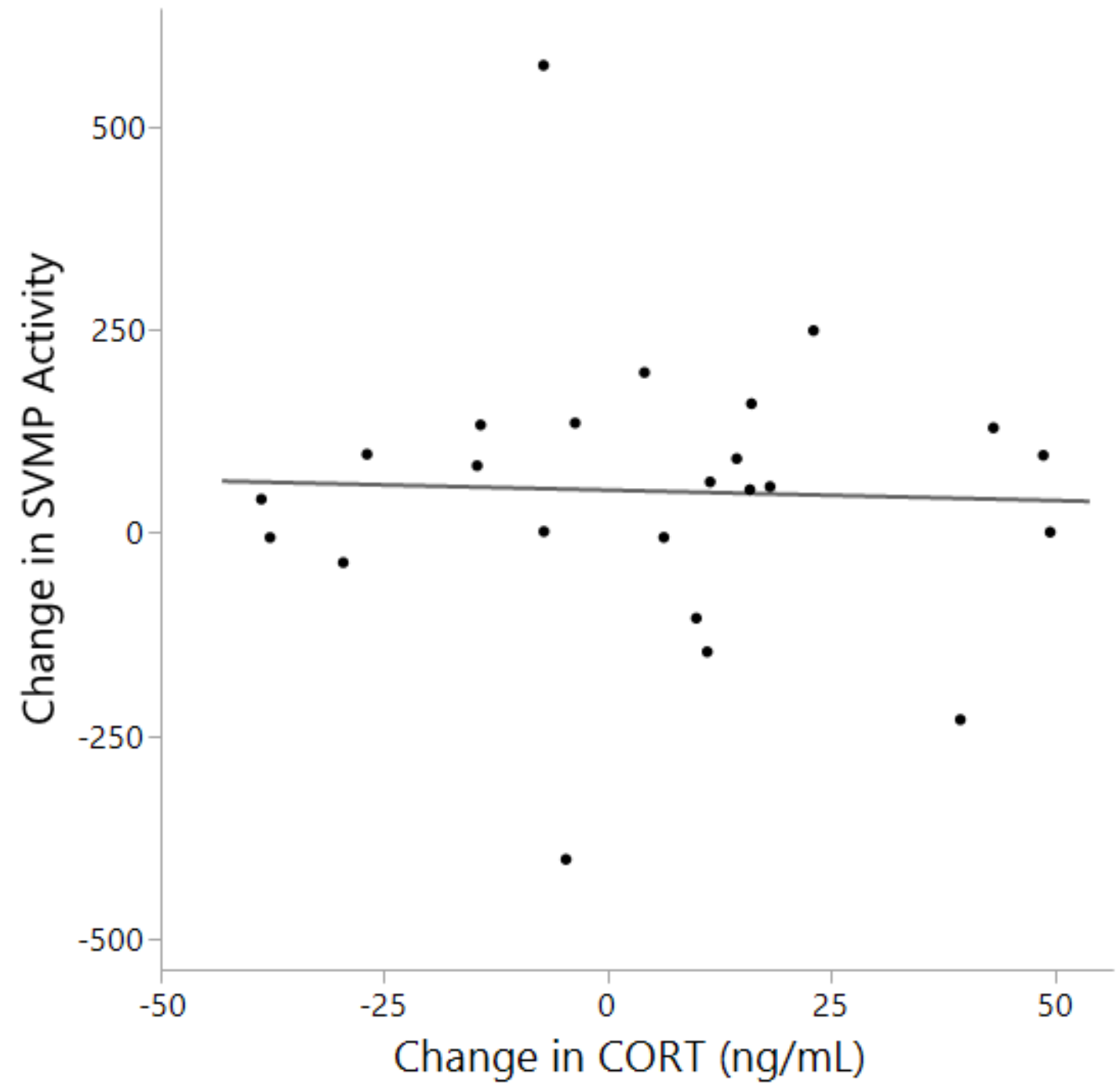

Figure A11. Change in SVMP activity from pre-implant sampling to sample 2 in Crotalus helleri against change in baseline CORT. Change in baseline CORT between the samples was not a significant covariate. 


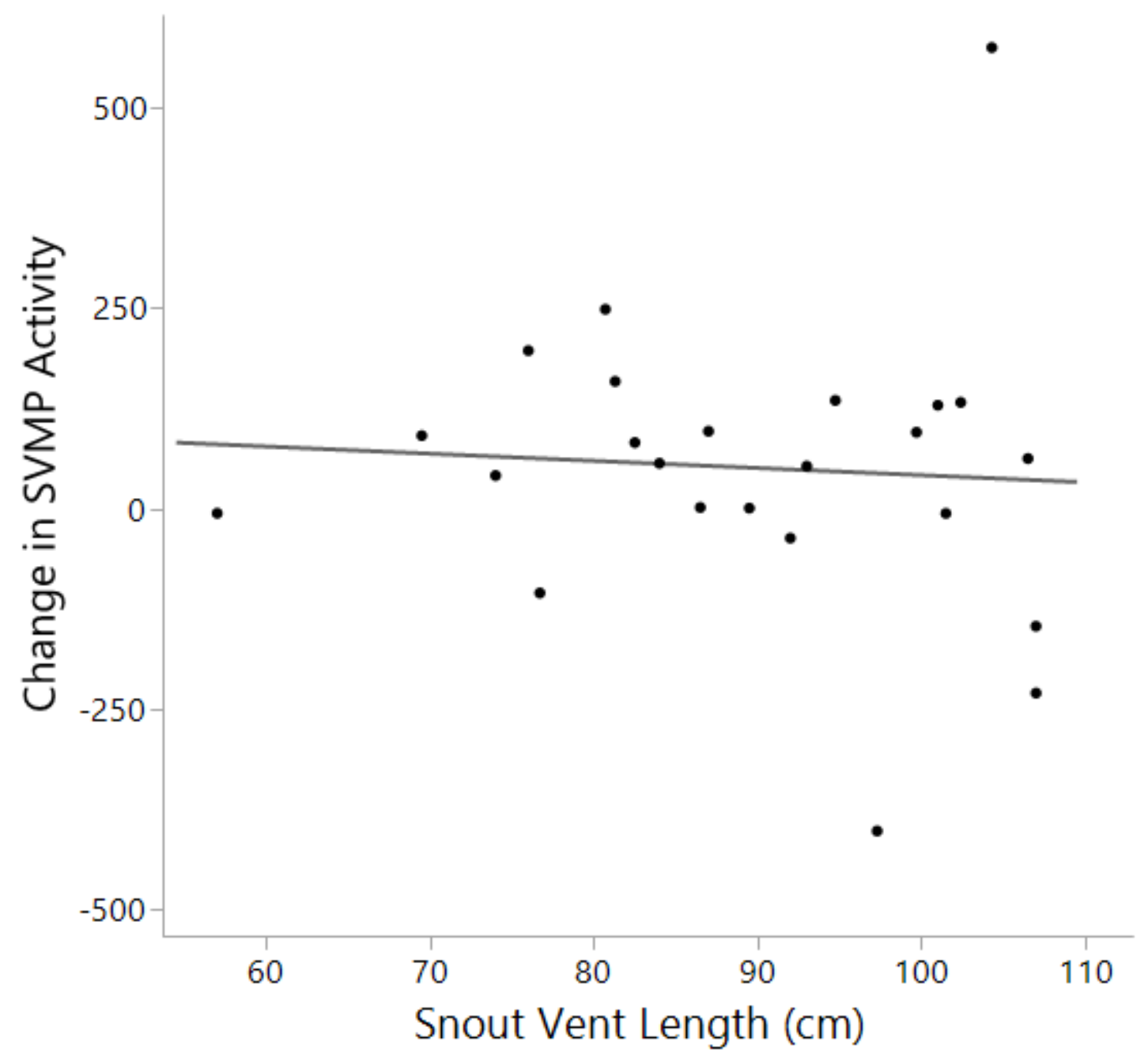

Figure A12. Change in SVMP activity from pre-implant sampling to 15 day after implant period in Crotalus helleri. SVL was not a significant covariate. 


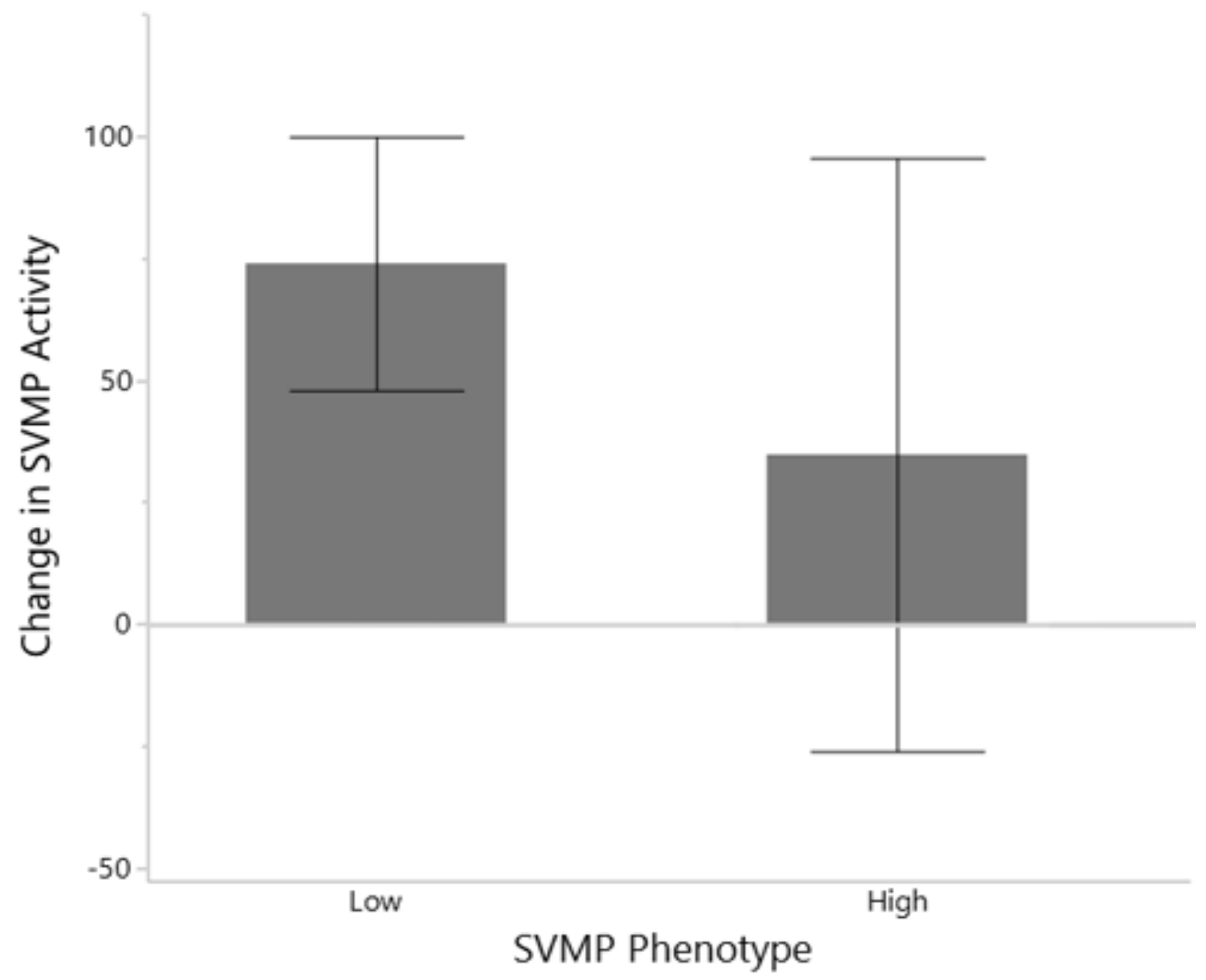

Figure A13. Change in SVMP activity from pre-implant sampling to sample2 in Low and High SVMP activity phenotype Crotalus helleri. Both phenotypes showed average increases in SVMP activity, but there was no effect of SVMP phenotype on change in SVMP activity during the initial implant period. Error bars are \pm 1 SEM. 


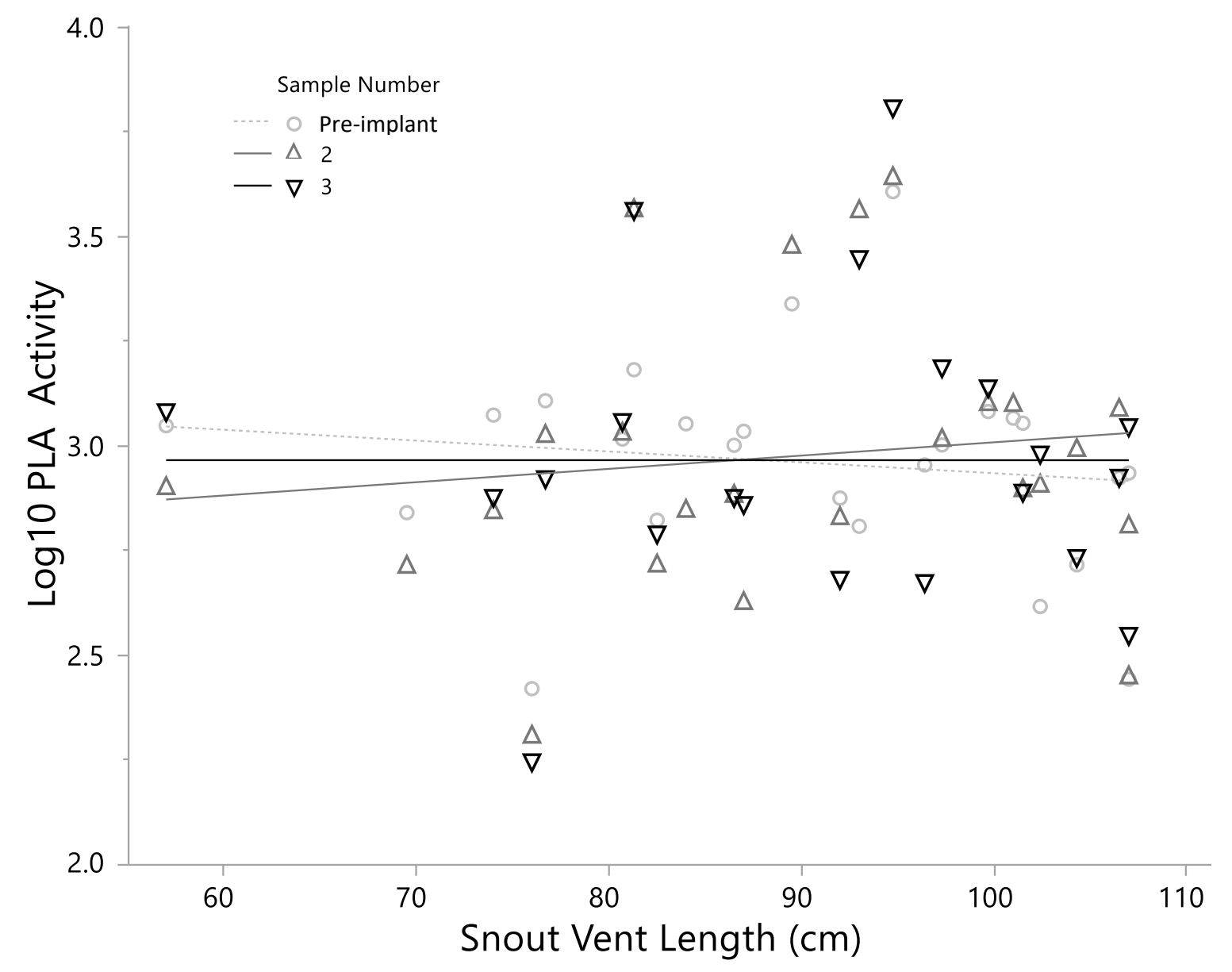

Figure A14. $\log _{10}$ transformed PLA 2 activity of Crotalus helleri plotted against SVL across all three sampling periods (in sequence from light to dark). SVL was not a significant covariate in the RM ANCOVA. Note clusters of dots along the $\mathrm{x}$-axis represent the samples from the same individual snake as SVL did not change appreciably during the study. Although all three samples from each snake are shown, each within-snake sample was not considered independent. The RM ANCOVA accounted for repetition within individuals, so there was no pseudoreplication in our dataset. 


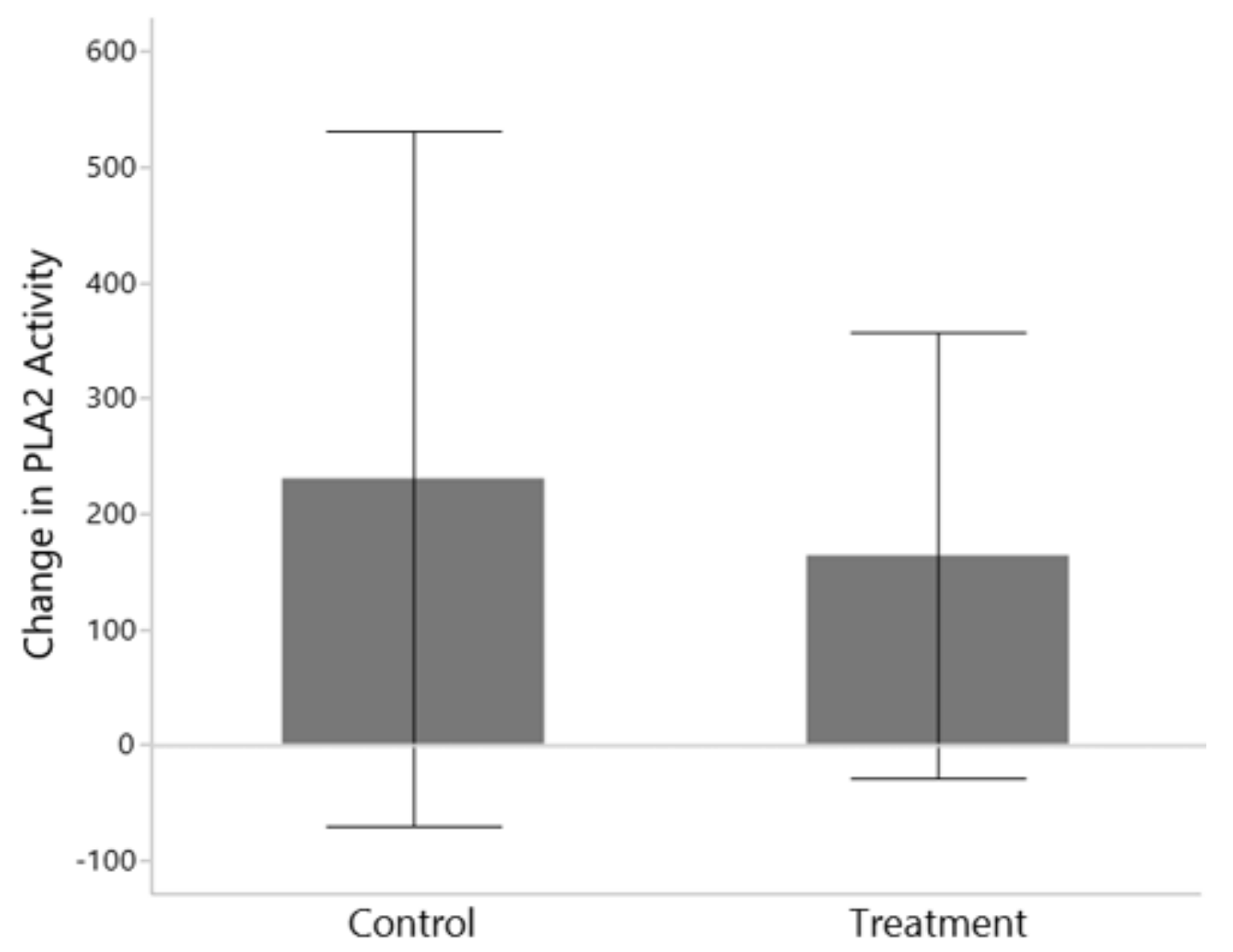

Figure A15. Histogram showing mean change in PLA 2 activity from pre-implant sampling to the second period (approximately 15 days after implants) in control and CORT-treated Crotalus helleri. Both control and treatment groups showed overall average increases in activity over the period. There was no effect of treatment on change in PLA 2 activity in an ANCOVA. Error bars are \pm 1 SEM. 


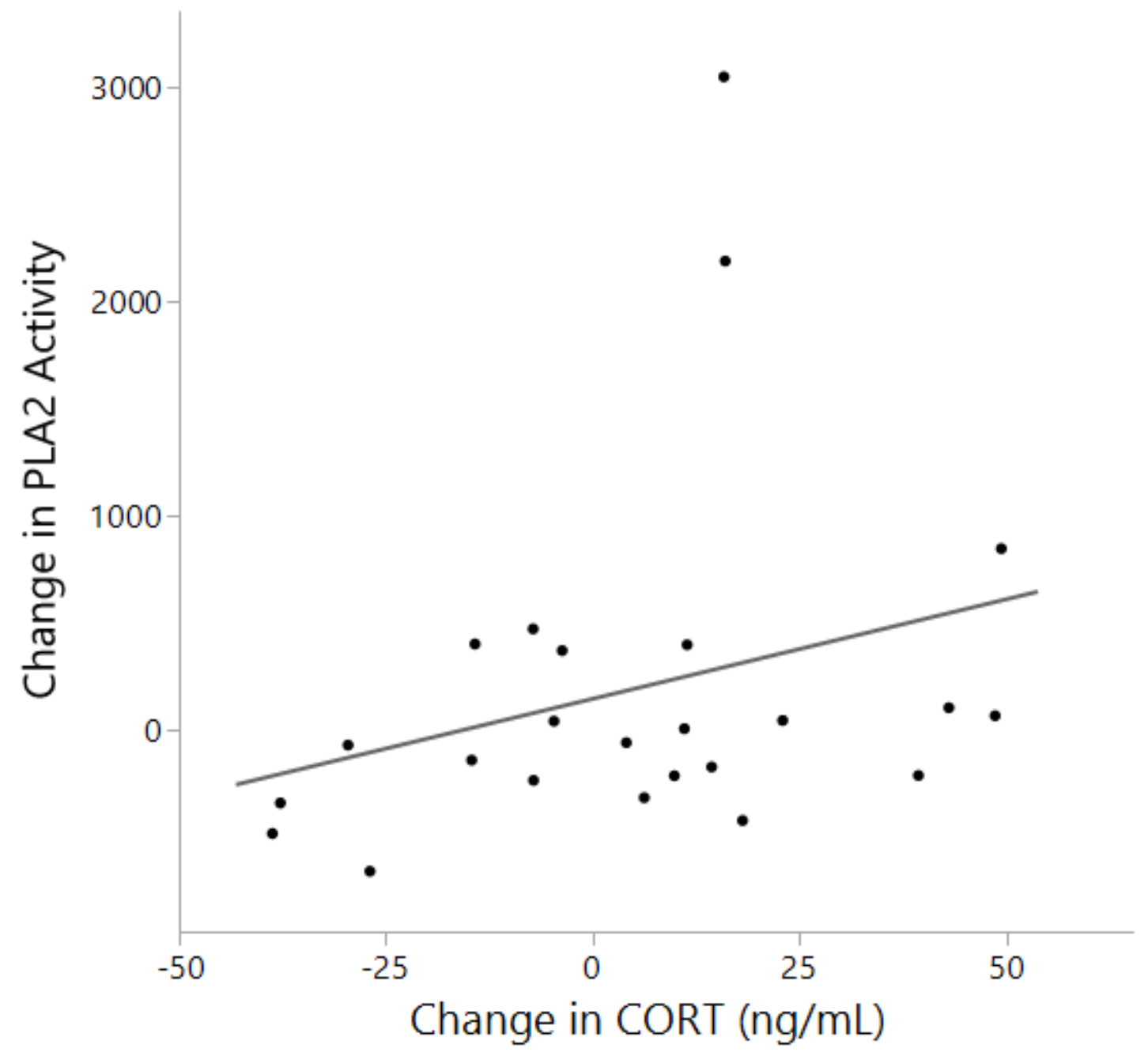

Figure A16. Change in $\mathrm{PLA}_{2}$ activity from pre-implant sampling to 15 days after implant period in Crotalus helleri. Change in baseline CORT between the samples was not a significant covariate. 


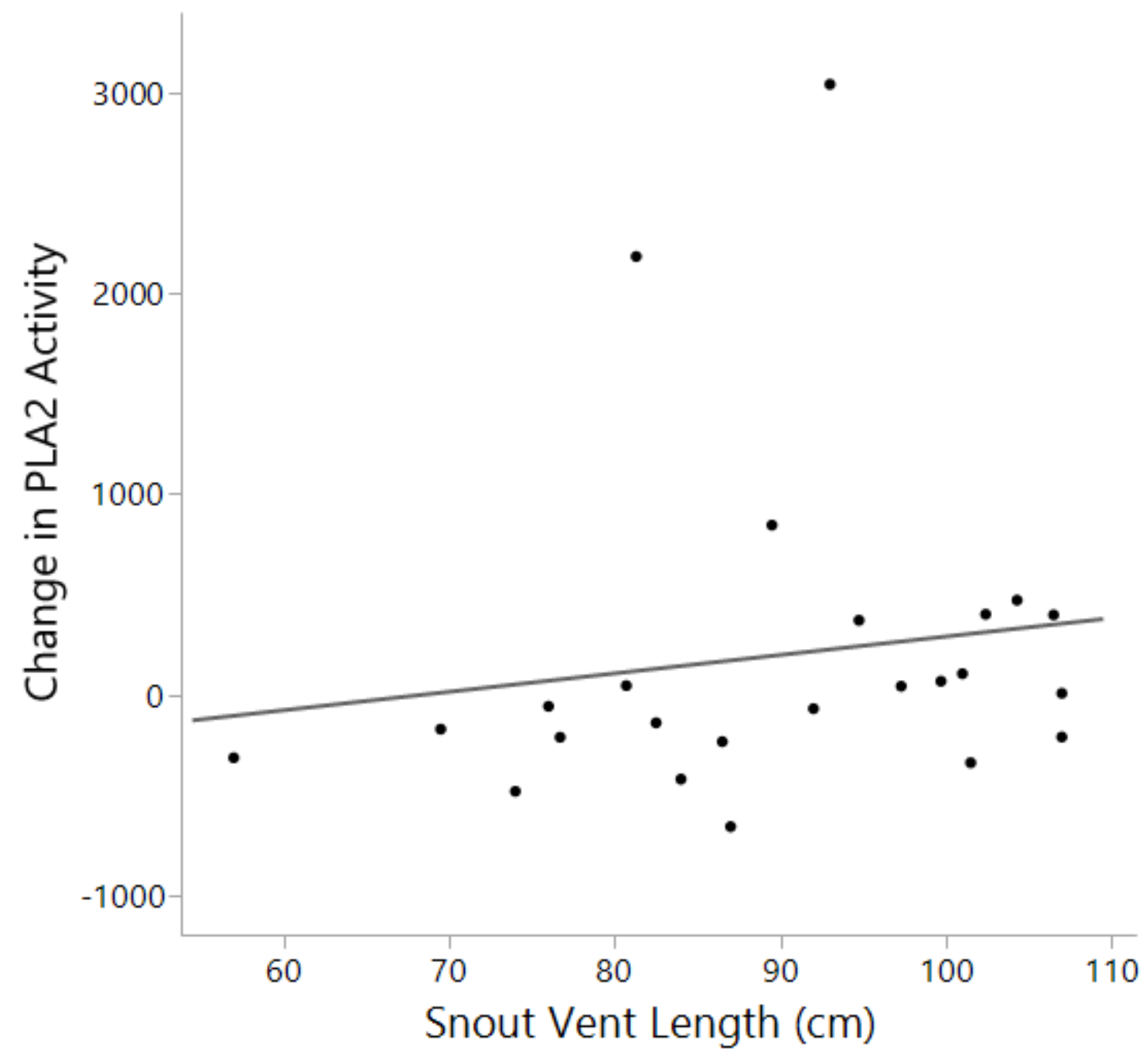

Figure A17. Change in $\mathrm{PLA}_{2}$ activity from pre-implant sampling to sample 2 in Crotalus helleri. SVL was not a significant covariate. 


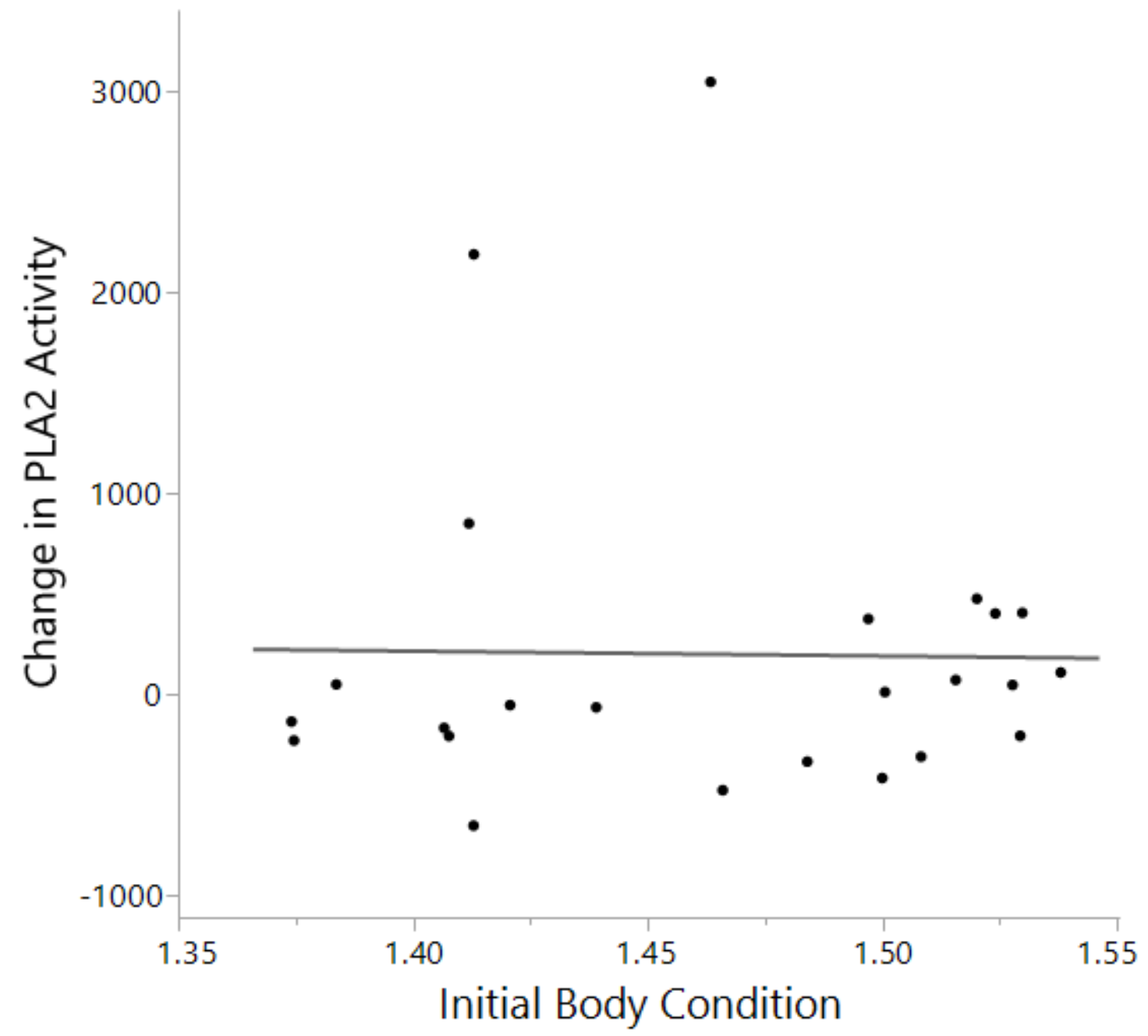

Figure A18. Change in PLA $_{2}$ activity from pre-implant sampling to sample 2 in Crotalus helleri. Initial body condition was a significant covariate, with snakes in higher body condition tending to decrease in PLA 2 activity from pre-implant to second sampling. 


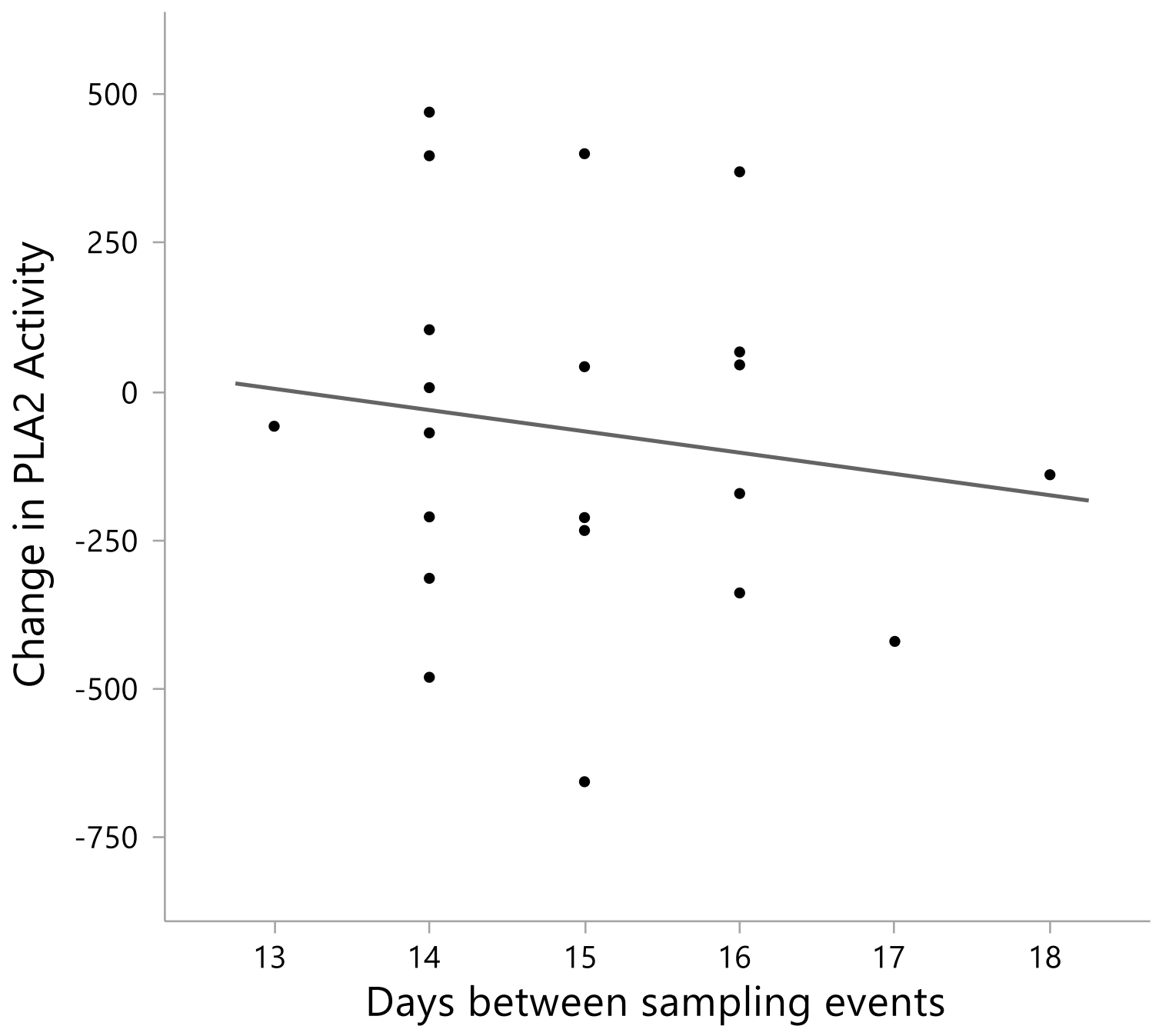

Figure A19. Change in PLA 2 activity from pre-implant sampling to sample 2 in Crotalus helleri, excluding three snakes with greater than 20 days between sampling events. Days between sampling was no longer a significant covariate. 


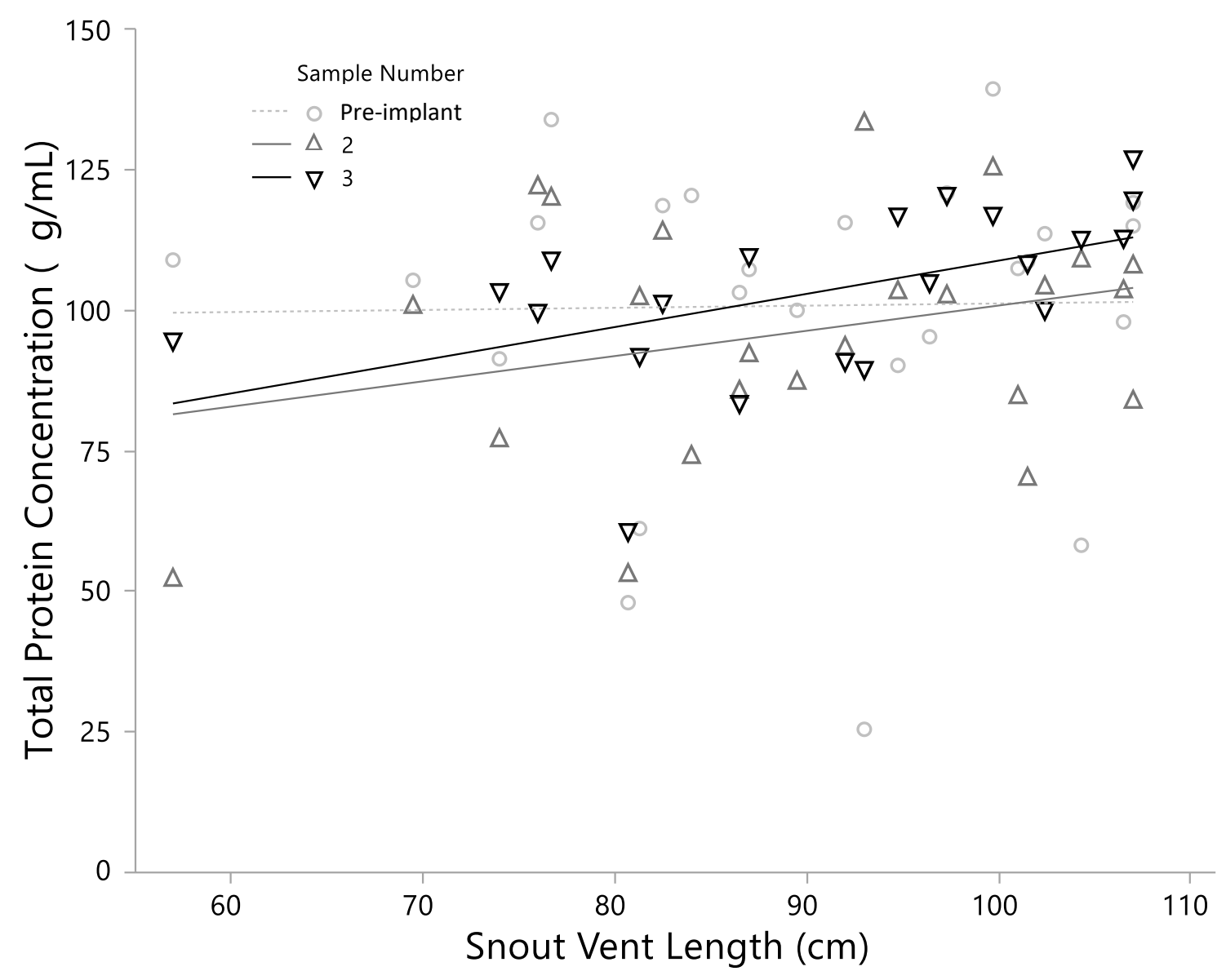

Figure A20. Total protein concentration in Crotalus helleri plotted against SVL across all three time periods (in sequence from light to dark). There was no relationship between SVL and total protein concentration. Note clusters of dots along the $\mathrm{x}$-axis represent the samples from the same individual snake as SVL did not change appreciably during the study. Although all three samples from each snake are shown, each within-snake sample was not considered independent. The RM ANCOVA accounted for repetition within individuals, so there was no pseudoreplication in our dataset. 


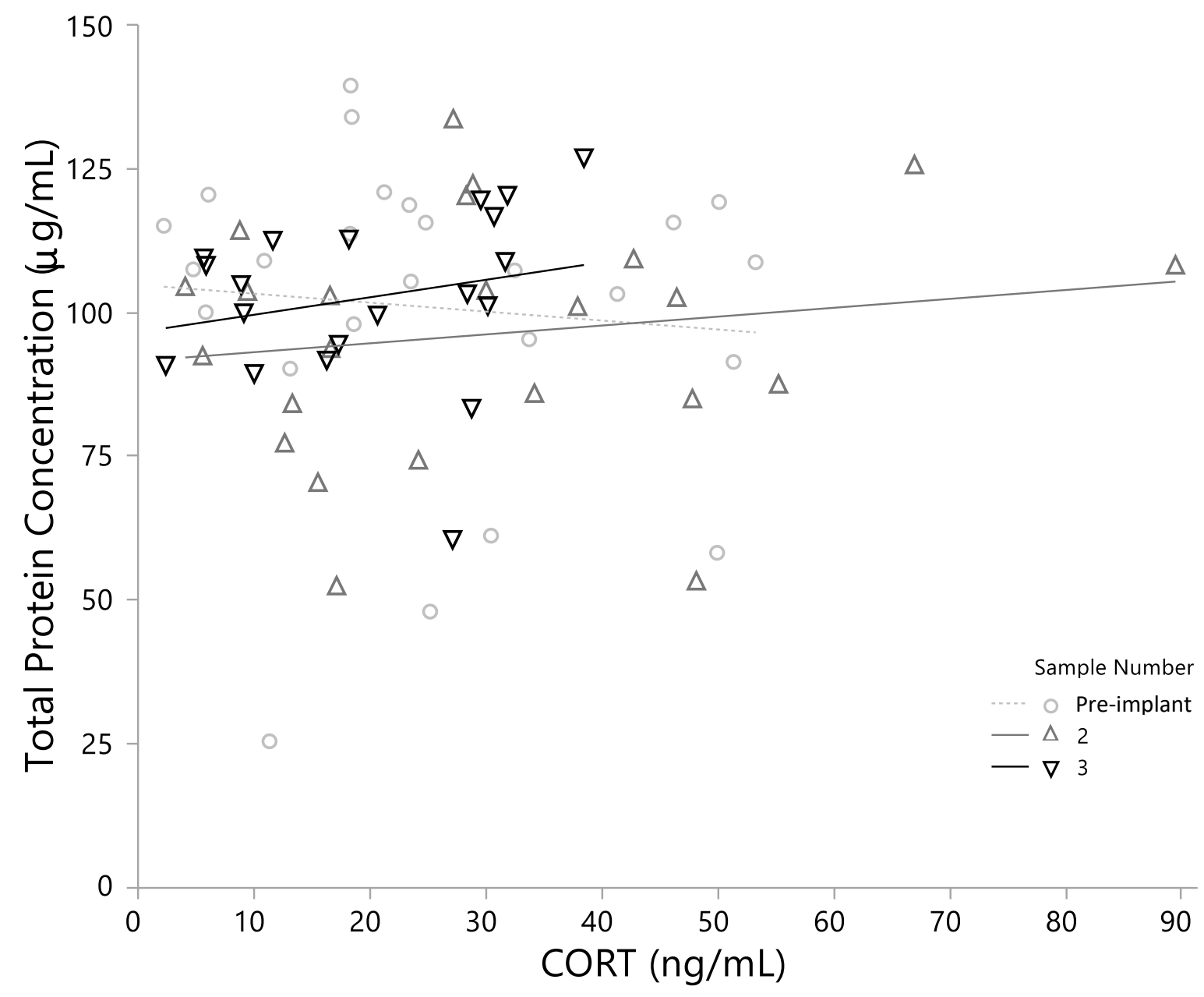

Figure A21. Total protein concentration of Crotalus helleri plotted against baseline CORT values across all three sampling periods (colored sequentially from light to dark). CORT was not a significant covariate in the RM ANCOVA. Although all three samples from each snake are shown, each within-snake sample was not considered independent. The RM ANCOVA accounted for repetition within individuals, so there was no pseudoreplication in our dataset. 


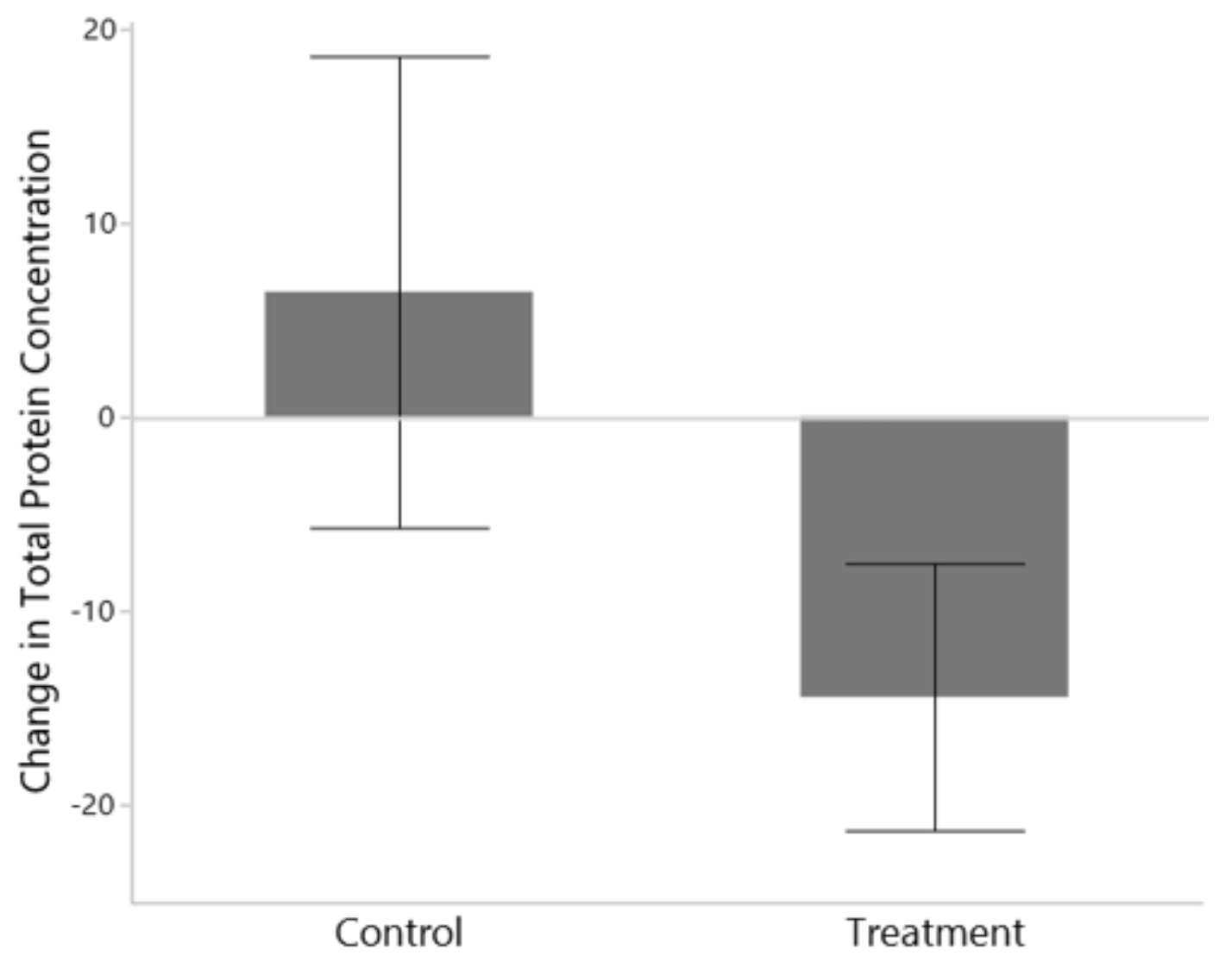

Figure A22. Histogram showing mean change in total protein concentration from pre-implant sampling to sample 2 (approximately 15 days after implants) in control and CORT-treated Crotalus helleri. While Control snakes showed overall average increases in total protein concentration and treatment snakes showed overall average decreases, there was no effect of treatment on change in total protein concentration in an ANCOVA. Error bars are \pm 1 SEM. 


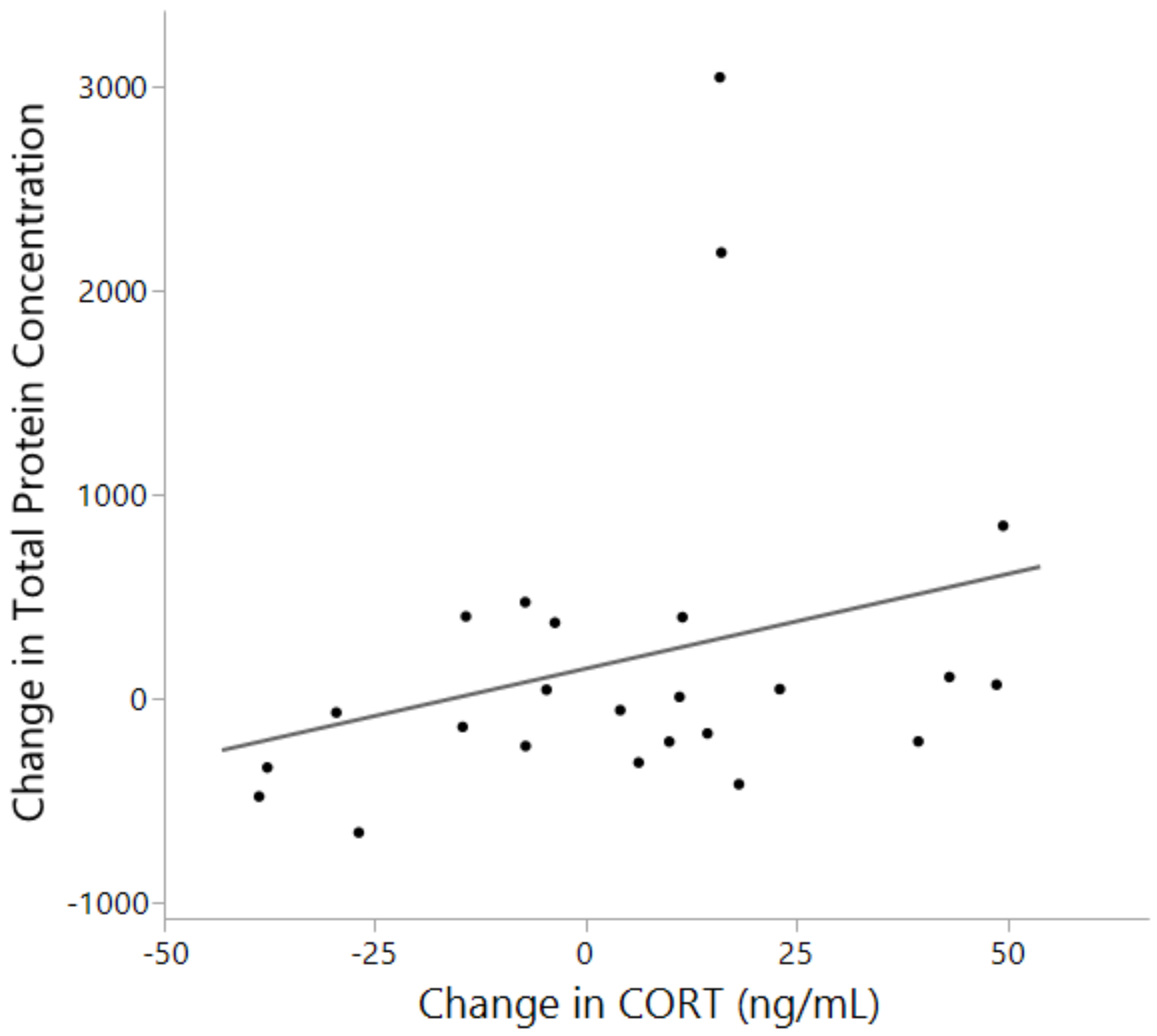

Figure A23. Change in total protein concentration from pre-implant sampling to sample 2 after implant in Crotalus helleri. Change in baseline CORT between samples was not a significant covariate. 


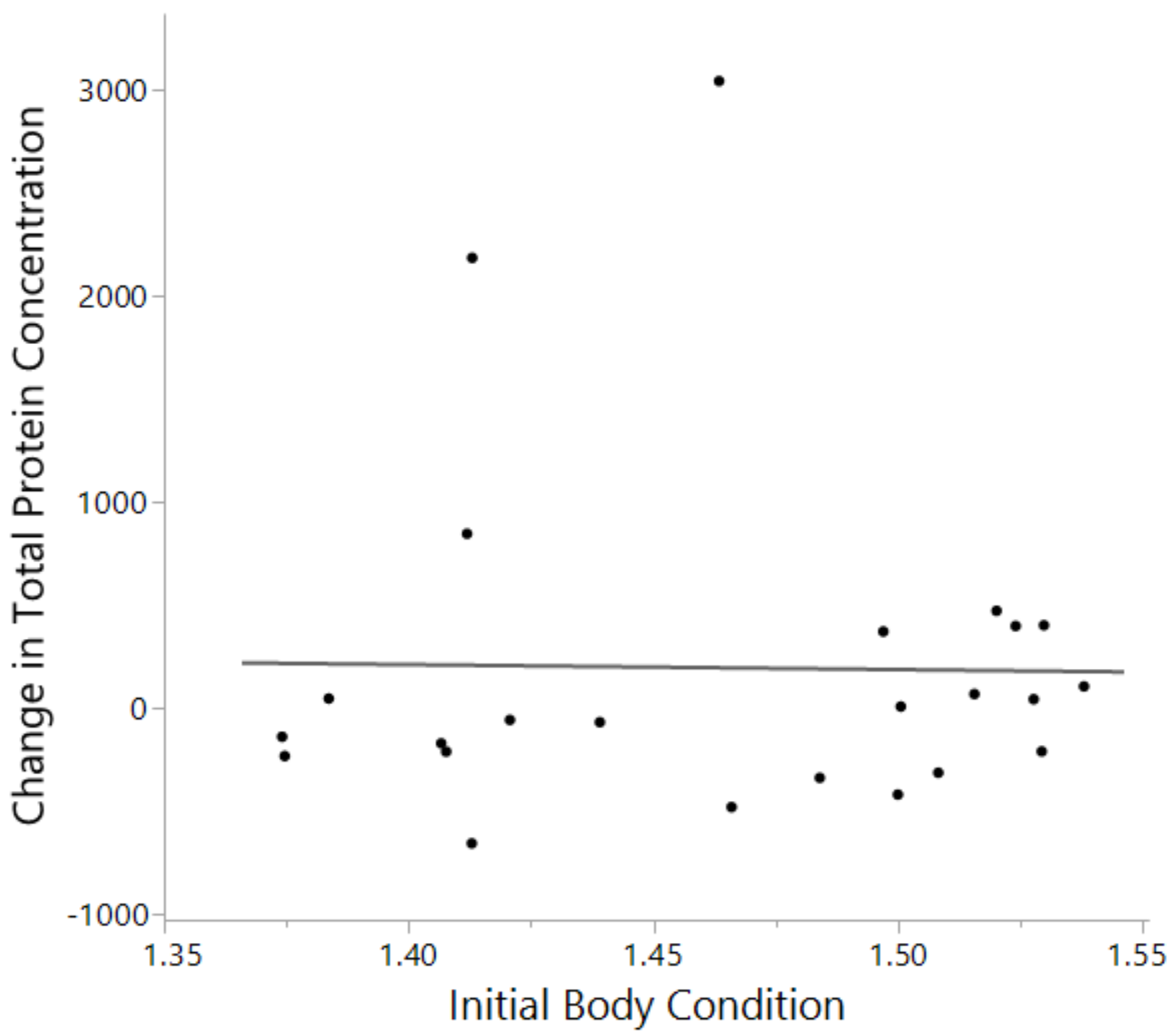

Figure A24. Change in total protein concentration from pre-implant sampling to sample 2 in Crotalus helleri. Initial body condition was not a significant covariate. 


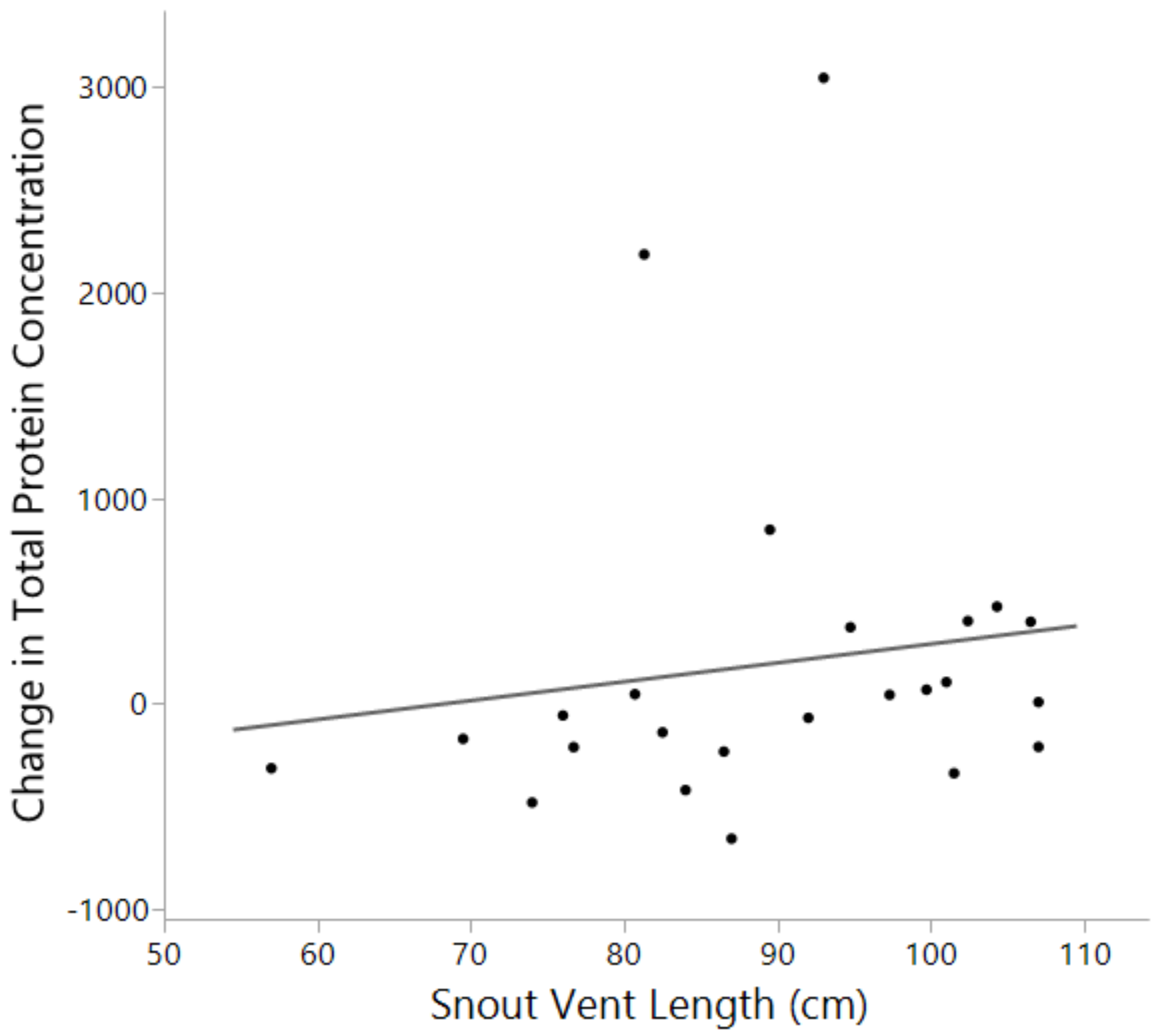

Figure A25. Change in total protein concentration from pre-implant sampling to sample 2 in Crotalus helleri. SVL was not a significant covariate. 


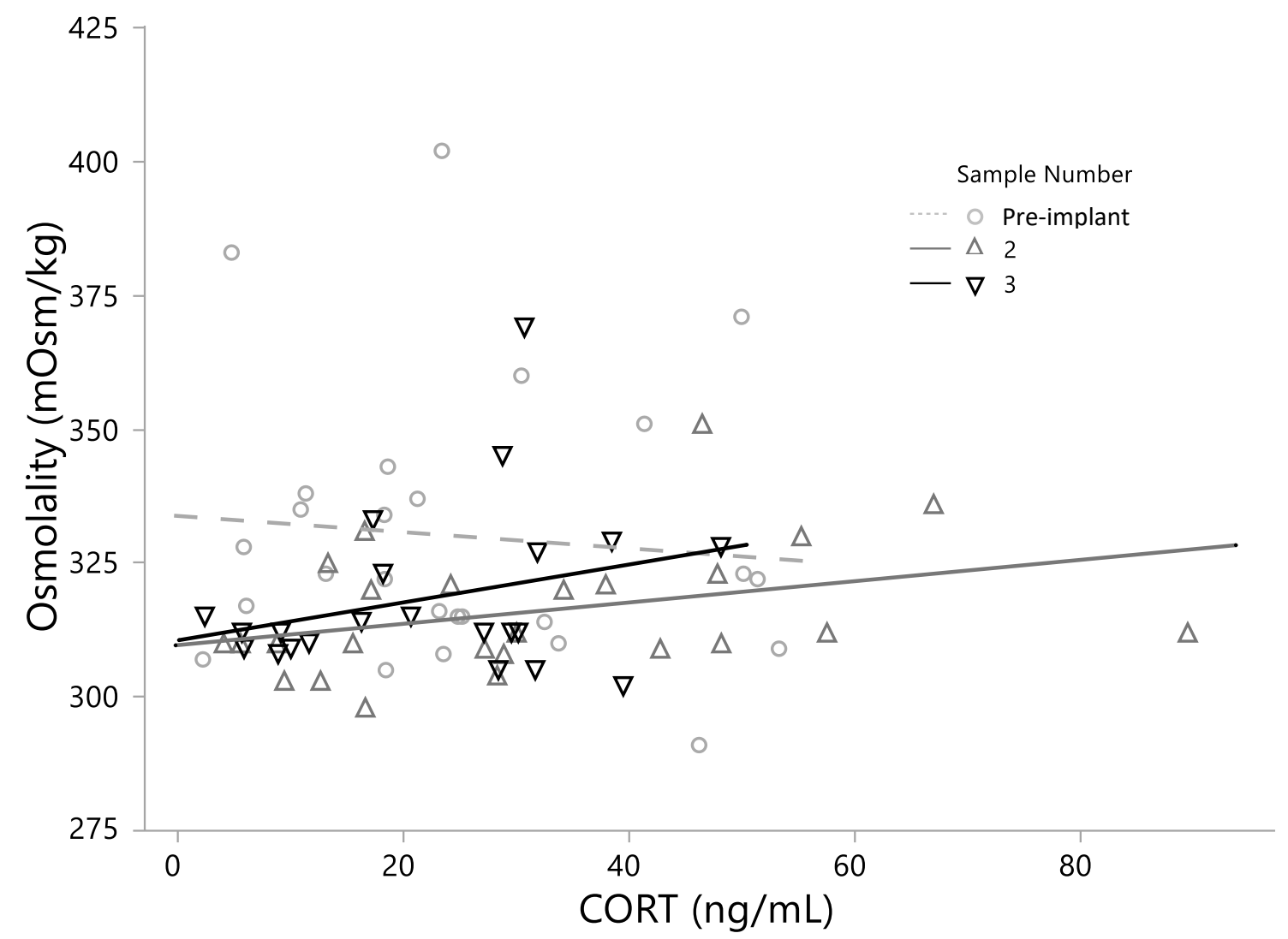

Figure A26. Plasma osmolality of Crotalus helleri plotted against baseline CORT across all three sampling periods (coded sequentially from light to dark). CORT was not a significant covariate in the RM ANCOVA. Although all three samples from each snake are shown, each within-snake sample was not considered independent. The RM ANCOVA accounted for repetition within individuals, so there was no pseudoreplication in our dataset. 


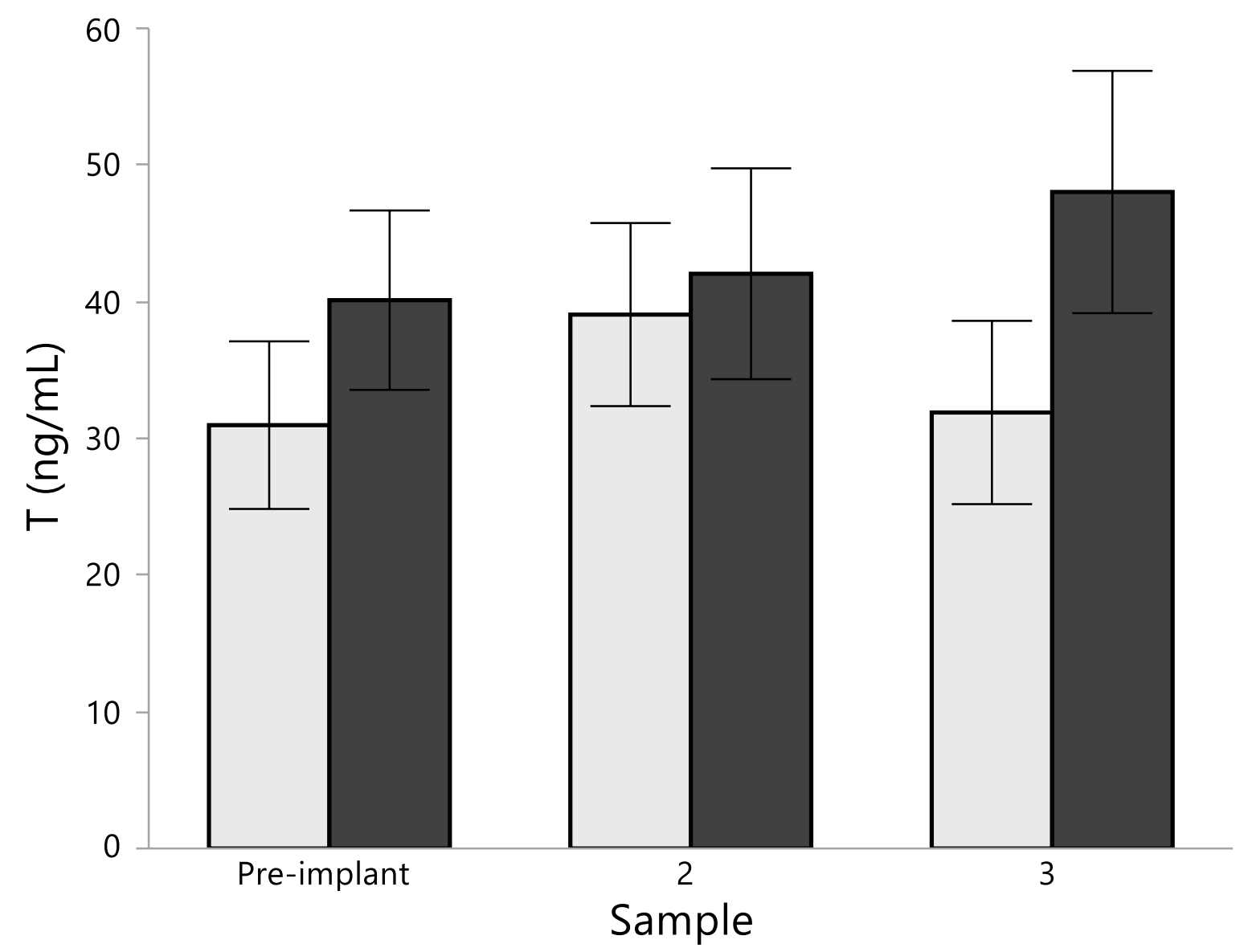

Figure A27. Mean plasma T of treatment (dark bars) and control (light bars) Crotalus helleri at each sampling period. T was not affected by treatment or sample number. Error bars are \pm 1 SEM. 


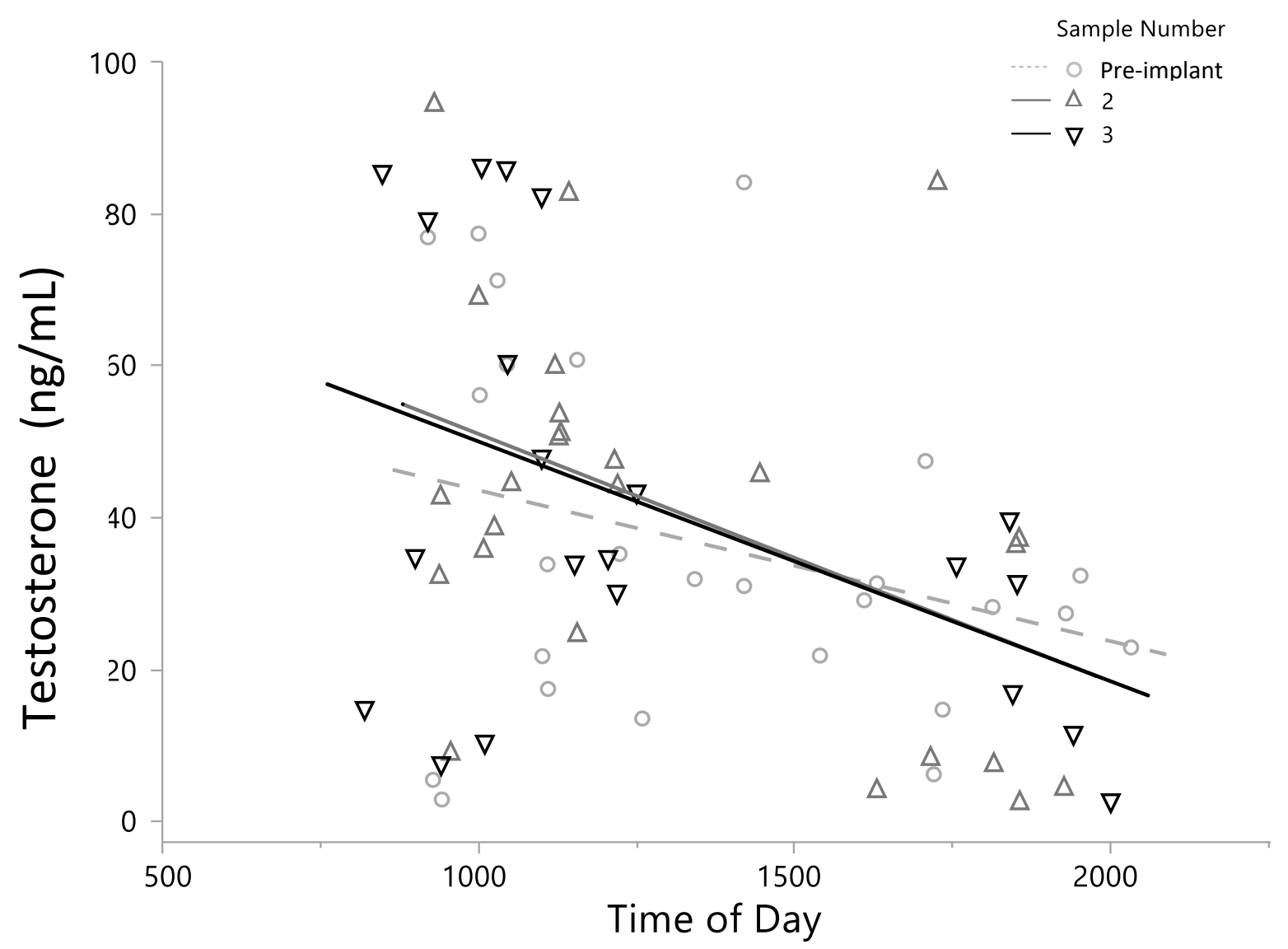

Figure A28. Plasma testosterone plotted against time of day for Crotalus helleri at each sample (coded sequentially from light to dark). There was a trend for testosterone to decrease late in the day. Although all three samples from each snake are shown, each within-snake sample was not considered independent. The RM ANCOVA accounted for repetition within individuals, so there was no pseudoreplication in our dataset. 


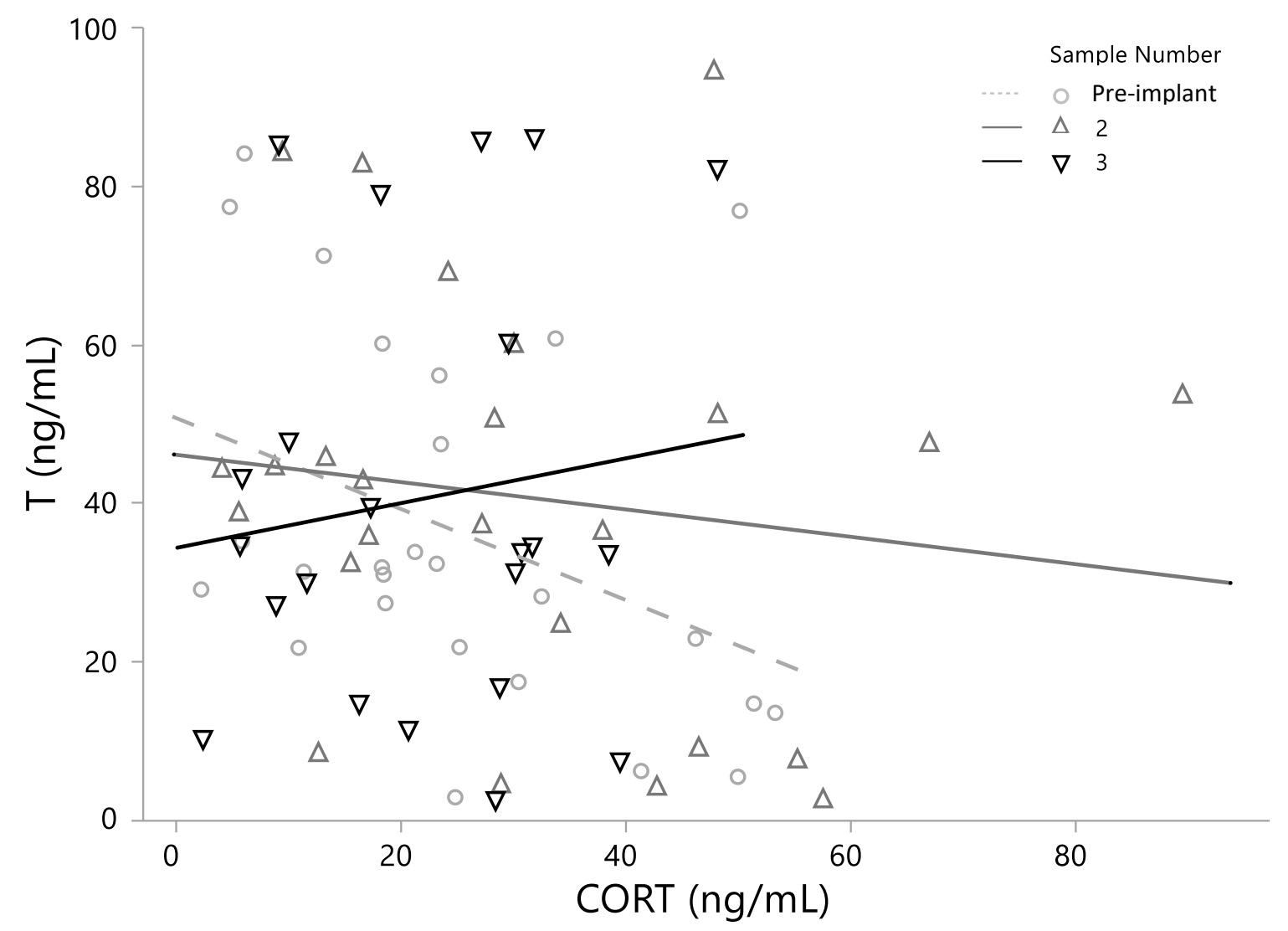

Figure A29. T plotted against CORT for Crotalus helleri across all three sampling periods (coded sequentially from light to dark). CORT was not a significant covariate in the RM ANCOVA. Although all three samples from each snake are shown, each within-snake sample was not considered independent. The RM ANCOVA accounted for repetition within individuals, so there was no pseudoreplication in our dataset. 


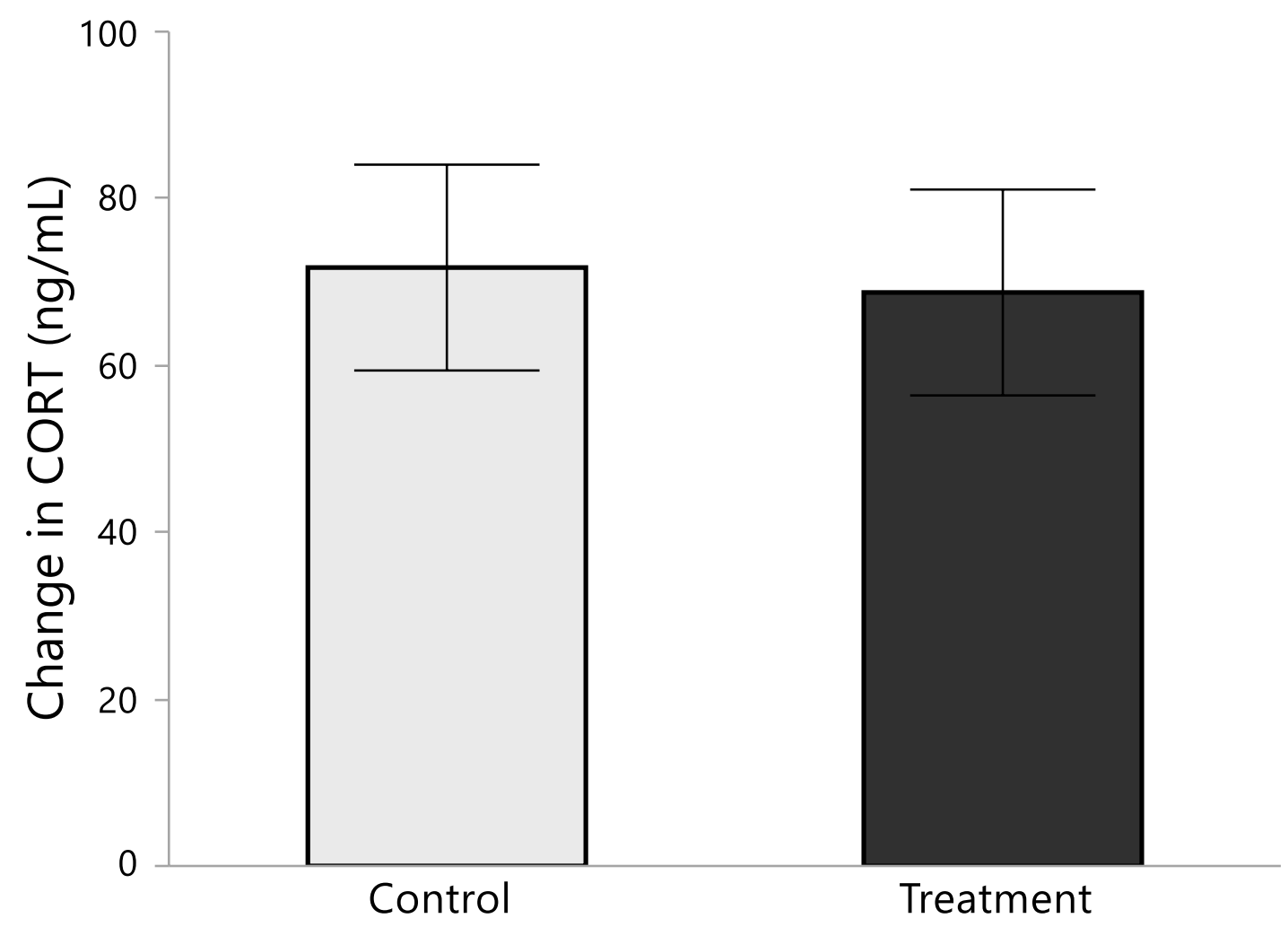

Figure A30. Magnitude of stress response in both control and treatment Crotalus helleri after one hour of acute confinement stress. There was no difference in stress response based on implant treatment. Error bars are \pm 1 SEM. 


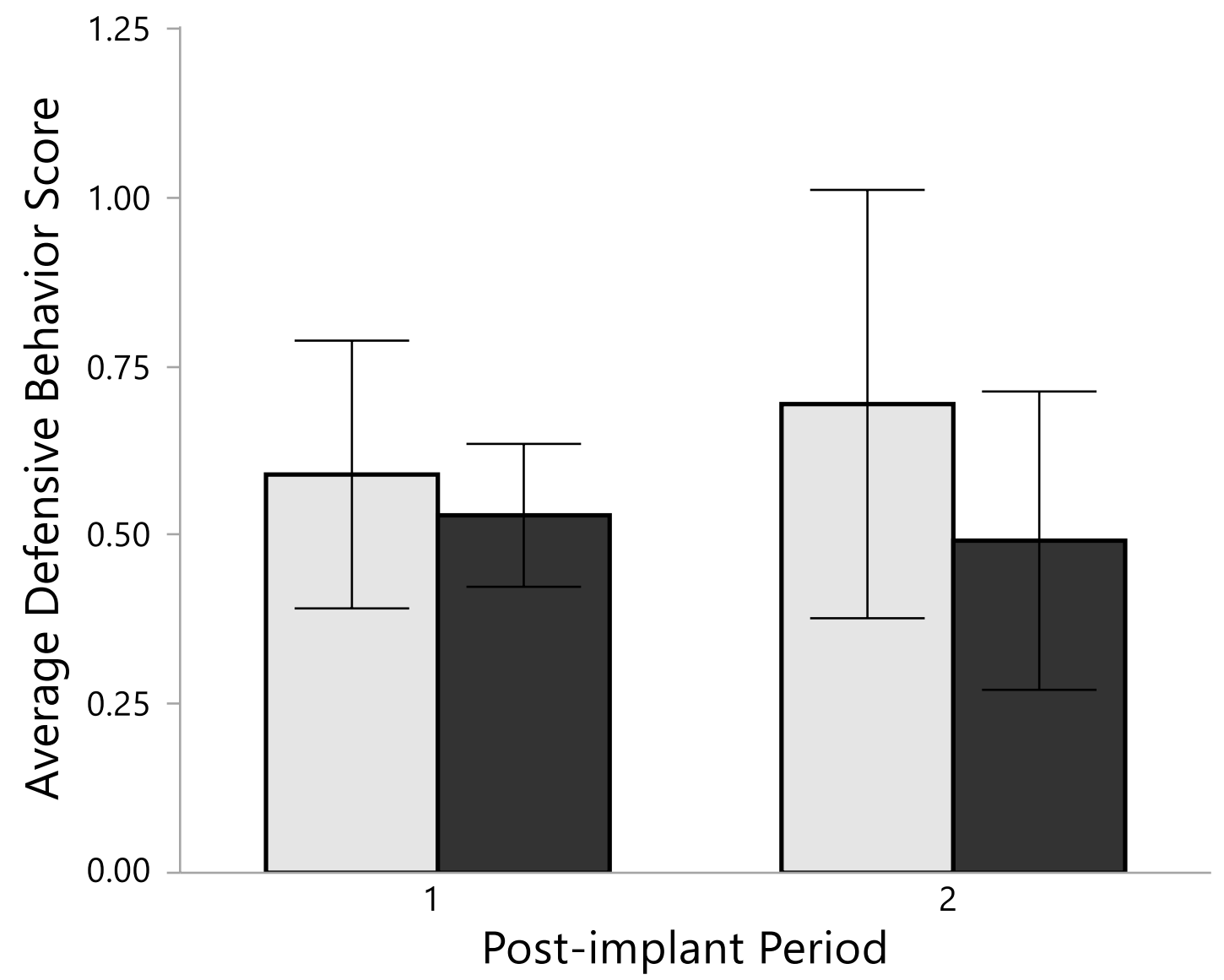

Figure A31. Average defensive behavior score during each tracking period for treatment (dark bars) and control (light bars) Crotalus helleri. Defensive behavior was not affected by treatment or sample number. Error bars are \pm 1 SEM. 


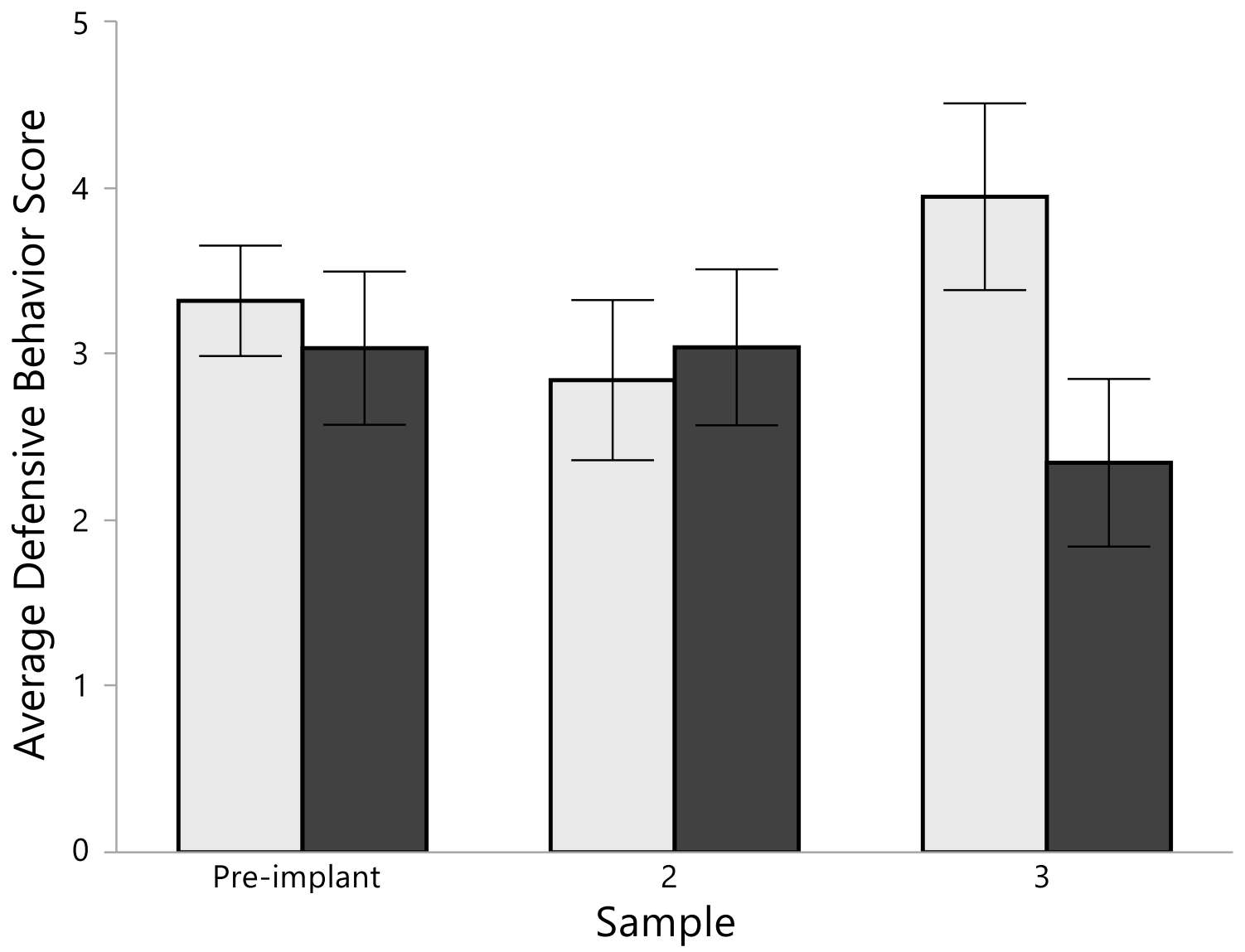

Figure A32. Average defensive behavior score during sampling for treatment (dark bars) and control (light bars) Crotalus helleri. Defensive behavior was not affected by treatment or sample number. Error bars are \pm 1 SEM. 


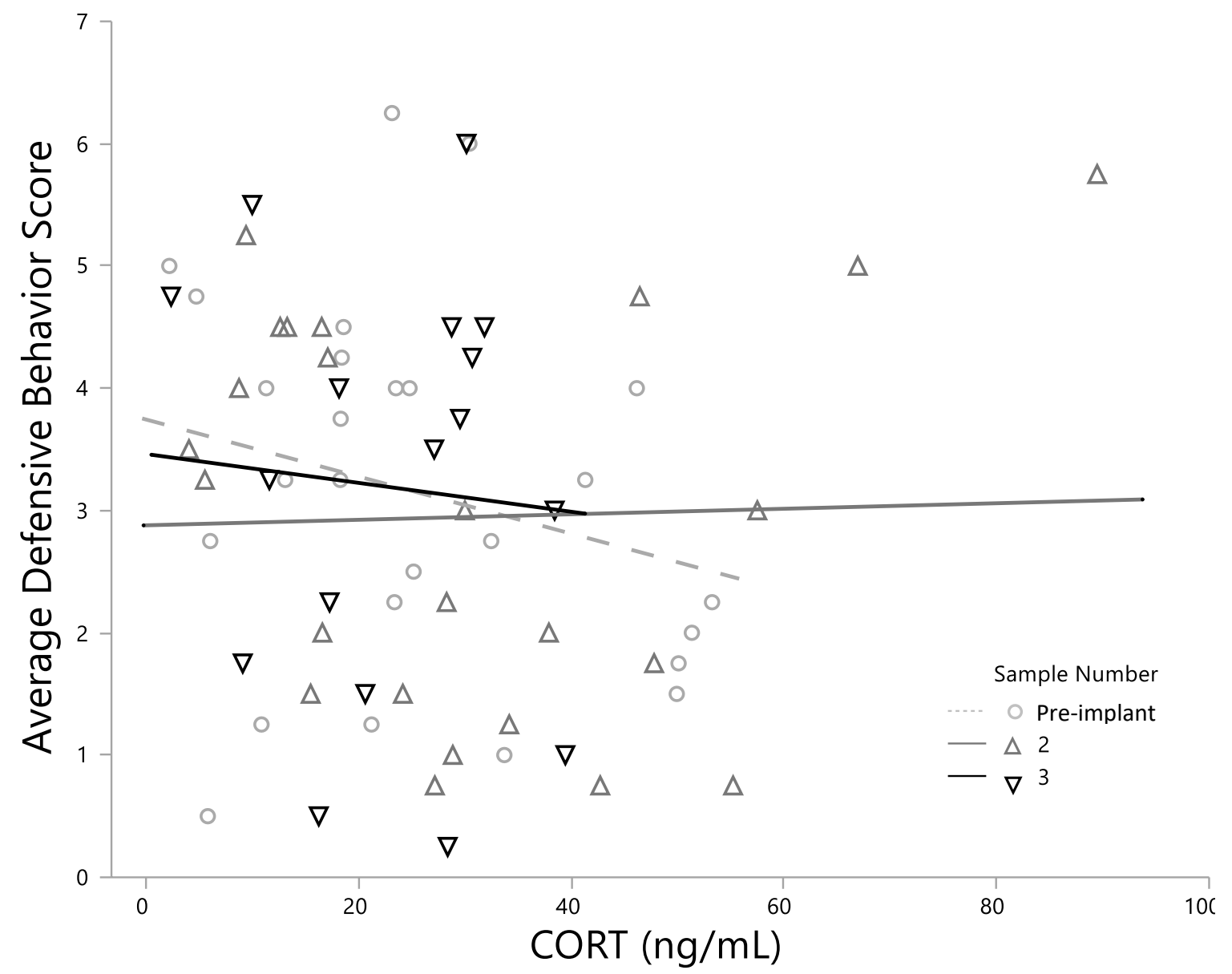

Figure A33. Average defensive behavior score during sampling of Crotalus helleri across three sampling periods (coded sequentially from light to dark). CORT was not a significant covariate in the RM ANCOVA. Although all three samples from each snake are shown, each within-snake sample was not considered independent. The RM ANCOVA accounted for repetition within individuals, so there was no pseudoreplication in our dataset. 


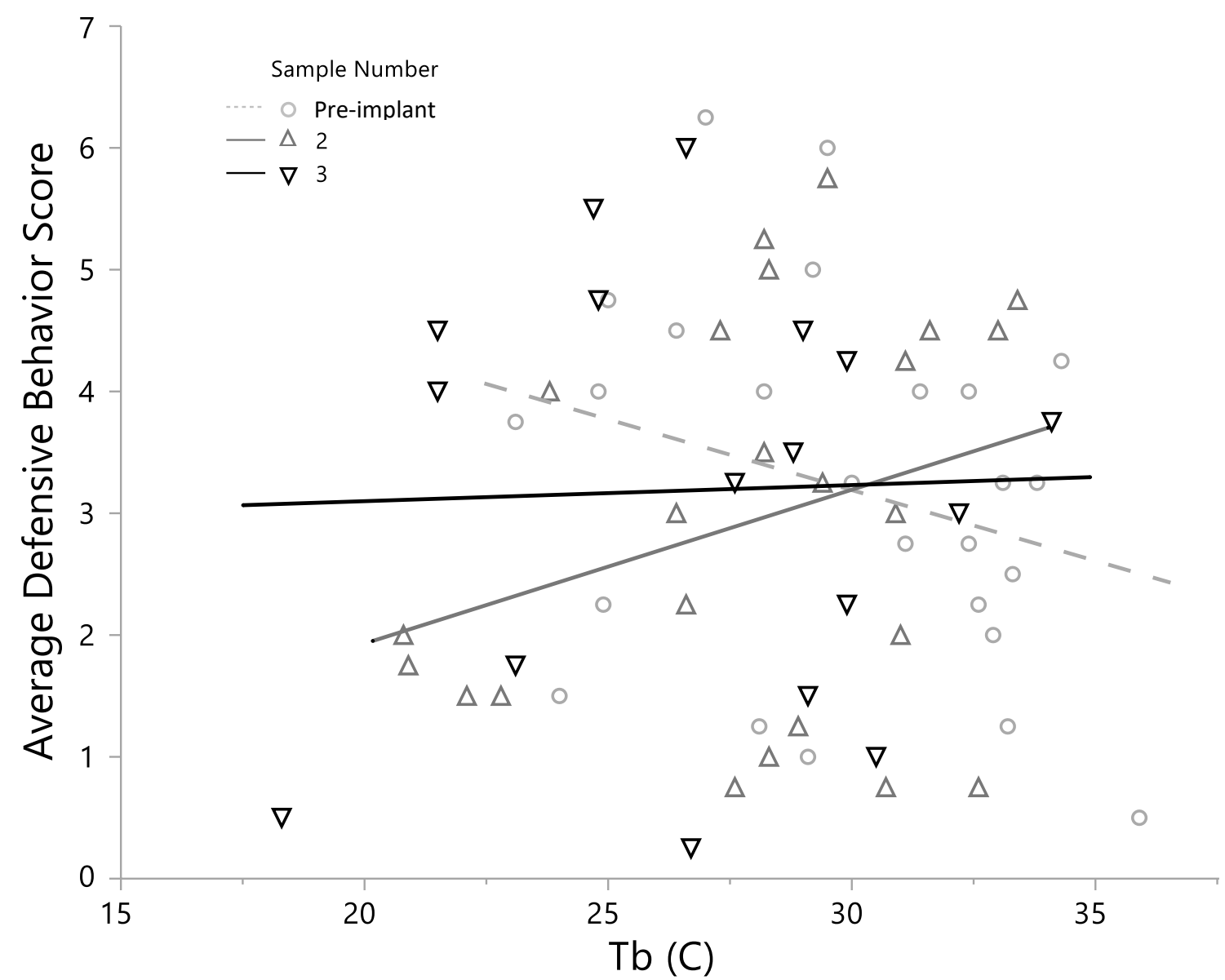

Figure A34. Average defensive behavior score during sampling of Crotalus helleri across three sampling periods (coded sequentially from light to dark). $T_{b}$ was not a significant covariate in the RM ANCOVA. Although all three samples from each snake are shown, each within-snake sample was not considered independent. The RM ANCOVA accounted for repetition within individuals, so there was no pseudoreplication in our dataset. 


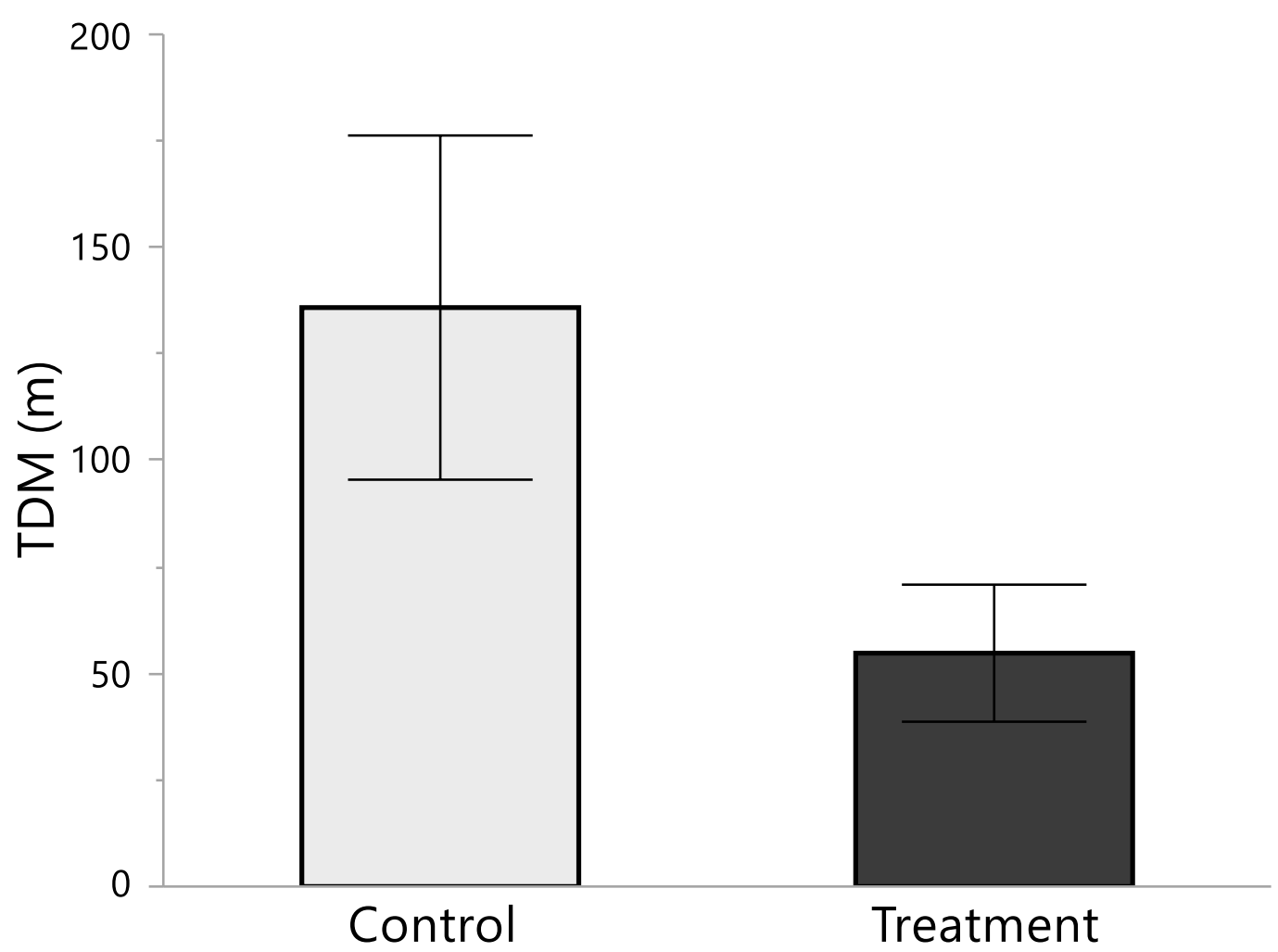

Figure A35. TDM of Crotalus helleri during the pre-implant tracking period. TDM was not significantly different based on treatment group in the one-way ANOVA. Error bars are \pm 1 SEM. 


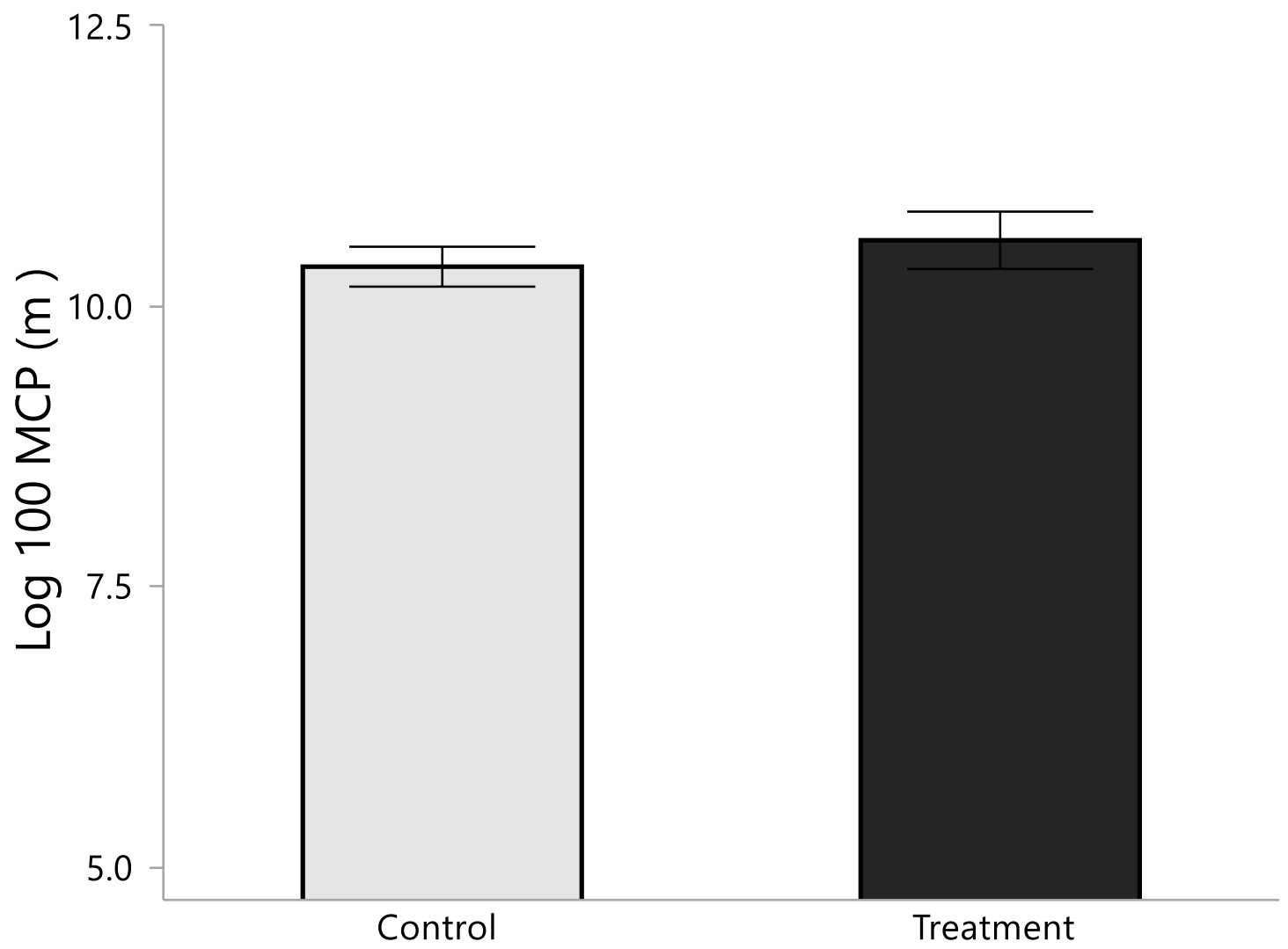

Figure A36. Home range size as measured by $100 \%$ MCP for control (light) and treatment (dark) Crotalus helleri over a three month period after implantation. There were no differences based on treatment. Error bars are \pm 1 SEM. 


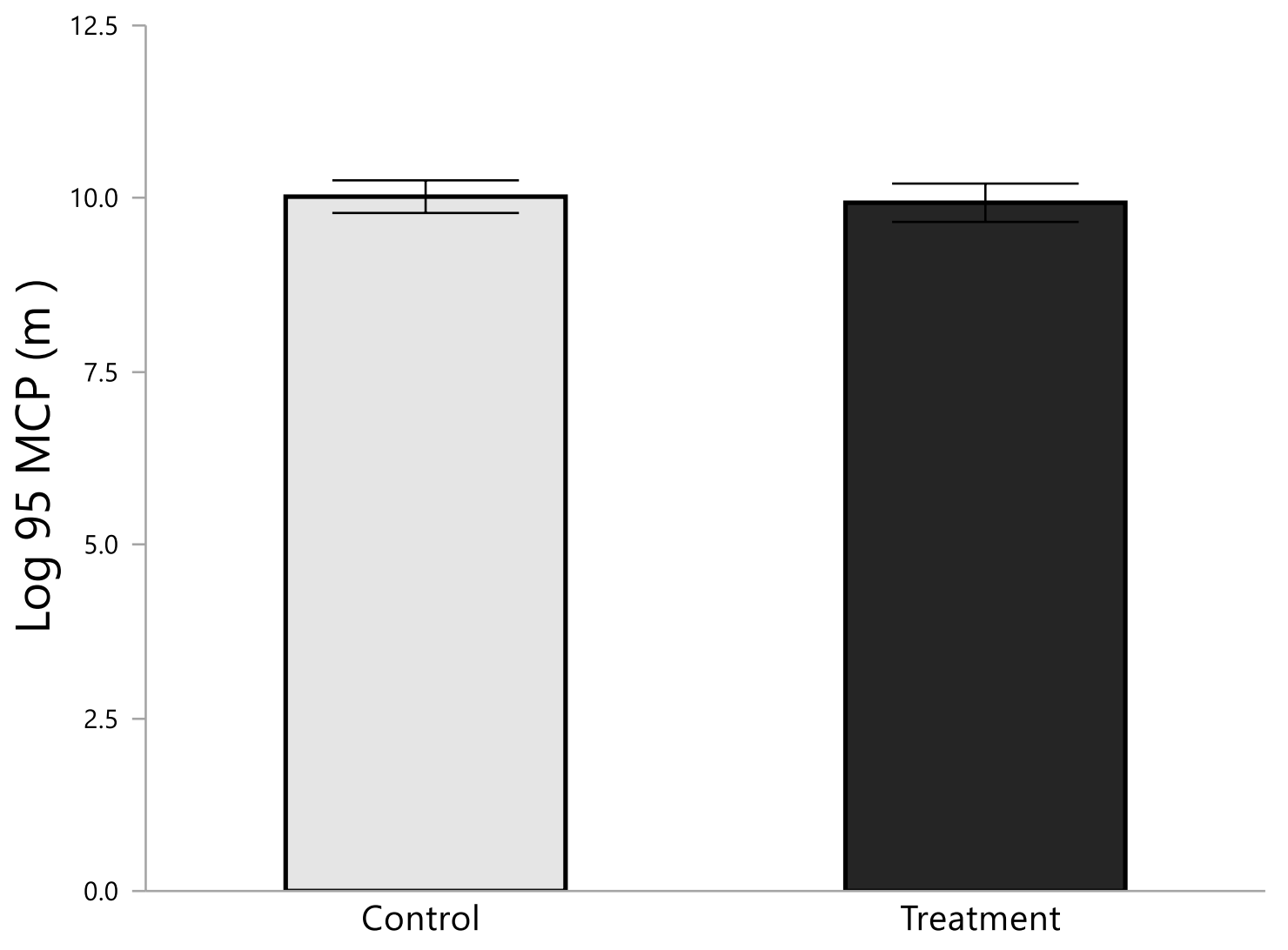

Figure A37. Home range size as measured by $95 \% \mathrm{MCP}$ for control (light) and treatment (dark) Crotalus helleri over a three month period after implantation. There were no differences based on treatment. Error bars are \pm 1 SEM. 